University of Tennessee Health Science Center

UTHSC Digital Commons

\title{
Craniofacial Changes Following Nonextraction Orthodontic Treatment: A Long-Term Cephalometric Analysis
}

James Austin Rahaim

University of Tennessee Health Science Center

Follow this and additional works at: https://dc.uthsc.edu/dissertations

Part of the Orthodontics and Orthodontology Commons

\section{Recommended Citation}

Rahaim, James Austin , "Craniofacial Changes Following Nonextraction Orthodontic Treatment: A LongTerm Cephalometric Analysis" (2011). Theses and Dissertations (ETD). Paper 208. http://dx.doi.org/ 10.21007/etd.cghs.2011.0256.

This Thesis is brought to you for free and open access by the College of Graduate Health Sciences at UTHSC Digital Commons. It has been accepted for inclusion in Theses and Dissertations (ETD) by an authorized administrator of UTHSC Digital Commons. For more information, please contact jwelch30@uthsc.edu. 


\title{
Craniofacial Changes Following Nonextraction Orthodontic Treatment: A Long- Term Cephalometric Analysis
}

\begin{abstract}
Long-term posttreatment cephalometric changes from late adolescence into early adulthood were analyzed in this study. Lateral cephalometric radiographs from a sample of 30 Class II division 1 Caucasian females treated without extractions were evaluated at posttreatment (mean age $=15.9$ years) and recall (mean age $=28.3$ years). All of the subjects were treated in the private practice of a single, experienced practitioner. The cephalograms were examined to investigate changes in the cranial base, midface, maxilla, mandible, maxillomandibular relationships, dental relationships, and the soft tissue profile that occurred at an average of 12.4 years posttreatment. Descriptive and inferential statistics were calculated to see whether the posttreatment changes were statistically significantly different from zero.
\end{abstract}

Significant posttreatment change $(P<0.0001)$ occurred for most skeletal measurements, and this was primarily attributed to late adolescent growth. Total mandibular length increased (Cd-Gn) by $6.6 \mathrm{~mm}$ on average, and total downward and forward directional growth of the maxilla (Se-A) was $4.3 \mathrm{~mm}$ on average. Overall, late mandibular growth after adolescence exceeded late growth in the maxilla by nearly twice as much, which was confirmed by an increase in SNA Angle by approximately 0.4 degrees and an increase in SNB Angle by approximately 0.8 degrees. Upper Anterior Facial Height increased by $3.1 \mathrm{~mm}$, and Lower Anterior Facial Height increased by $4.3 \mathrm{~mm}$, making the total increase in the vertical dimension of the anterior face greater than $7 \mathrm{~mm}$.

Dentally, the upper and lower incisors experienced significant uprighting after treatment, which was confirmed by decreases in U1-SN, U1-NA, IMPA, and L1-NB angles. Overbite and overjet increased by 0.9 $\mathrm{mm}$ and $1.0 \mathrm{~mm}$, respectively. Maxillary and mandibular arch lengths decreased by $1.2 \mathrm{~mm}$ and $1.7 \mathrm{~mm}$, respectively, and this was associated with mesial movement of the maxillary and mandibular first molars.

Soft tissue profiles became progressively more flattened after treatment. This was disclosed by an increase in $Z$ Angle by 4.5 degrees and increased retrusion of the upper and lower lips relative to the $E$ Plane. The nose and soft tissue chin continued to grow forward after treatment (NaPerp-Pr increased by $1.9 \mathrm{~mm}$ and $\mathrm{W}$ point-Pg' increased by $1.5 \mathrm{~mm}$ ). The upper and lower lips drooped inferiorly by $1.7 \mathrm{~mm}$ and $2.3 \mathrm{~mm}$, respectively.

\section{Document Type}

Thesis

Degree Name

Master of Dental Science (MDS)

Program

Orthodontics

Research Advisor

Edward F. Harris, Ph.D.

Keywords

Cephalometric, Long-Term, Nonextraction, Orthodontic 


\section{Subject Categories}

Dentistry | Medicine and Health Sciences | Orthodontics and Orthodontology 


\title{
CRANIOFACIAL CHANGES FOLLOWING NONEXTRACTION ORTHODONTIC TREATMENT: \\ A LONG-TERM CEPHALOMETRIC ANALYSIS
}

\author{
A Thesis \\ Presented for \\ The Graduate Studies Council \\ The University of Tennessee \\ Health Science Center
}

\author{
In Partial Fulfillment \\ Of the Requirements for the Degree \\ Master of Dental Science \\ From The University of Tennessee
}

By

James Austin Rahaim, D.M.D.

May 2011 
Copyright (? 2011 by James Austin Rahaim.

All rights reserved. 


\section{ACKNOWLEDGEMENTS}

I would like to thank my family, and most of all, my wife Reagyn, for their emotional support throughout my graduate education. I would also like to thank Dr. Edward Harris for his expertise, continued guidance, and leadership throughout the writing of this thesis. Without his help and guidance, this study would have not been possible. I would like to thank Dr. Richard Williams for providing his patient records for this study, and also his orthodontic staff for their support in helping myself and Dr. Lina Kulkarni to collect the records needed for this study. I would like to thank Dr. Williams and Dr. Jere Yates for serving on my thesis committee. Their direction and support were invaluable. 


\begin{abstract}
Long-term posttreatment cephalometric changes from late adolescence into early adulthood were analyzed in this study. Lateral cephalometric radiographs from a sample of 30 Class II division 1 Caucasian females treated without extractions were evaluated at posttreatment (mean age $=15.9$ years) and recall (mean age $=28.3$ years). All of the subjects were treated in the private practice of a single, experienced practitioner. The cephalograms were examined to investigate changes in the cranial base, midface, maxilla, mandible, maxillomandibular relationships, dental relationships, and the soft tissue profile that occurred at an average of 12.4 years posttreatment. Descriptive and inferential statistics were calculated to see whether the posttreatment changes were statistically significantly different from zero.
\end{abstract}

Significant posttreatment change $(\mathrm{P}<0.0001)$ occurred for most skeletal measurements, and this was primarily attributed to late adolescent growth. Total mandibular length increased (Cd-Gn) by $6.6 \mathrm{~mm}$ on average, and total downward and forward directional growth of the maxilla (Se-A) was $4.3 \mathrm{~mm}$ on average. Overall, late mandibular growth after adolescence exceeded late growth in the maxilla by nearly twice as much, which was confirmed by an increase in SNA Angle by approximately 0.4 degrees and an increase in SNB Angle by approximately 0.8 degrees. Upper Anterior Facial Height increased by $3.1 \mathrm{~mm}$, and Lower Anterior Facial Height increased by $4.3 \mathrm{~mm}$, making the total increase in the vertical dimension of the anterior face greater than $7 \mathrm{~mm}$.

Dentally, the upper and lower incisors experienced significant uprighting after treatment, which was confirmed by decreases in U1-SN, U1-NA, IMPA, and L1-NB angles. Overbite and overjet increased by $0.9 \mathrm{~mm}$ and $1.0 \mathrm{~mm}$, respectively. Maxillary and mandibular arch lengths decreased by $1.2 \mathrm{~mm}$ and $1.7 \mathrm{~mm}$, respectively, and this was associated with mesial movement of the maxillary and mandibular first molars.

Soft tissue profiles became progressively more flattened after treatment. This was disclosed by an increase in Z Angle by 4.5 degrees and increased retrusion of the upper and lower lips relative to the $\mathrm{E}$ Plane. The nose and soft tissue chin continued to grow forward after treatment (NaPerp-Pr increased by $1.9 \mathrm{~mm}$ and $\mathrm{W}$ point-Pg' increased by $1.5 \mathrm{~mm}$ ). The upper and lower lips drooped inferiorly by $1.7 \mathrm{~mm}$ and $2.3 \mathrm{~mm}$, respectively. 


\section{TABLE OF CONTENTS}

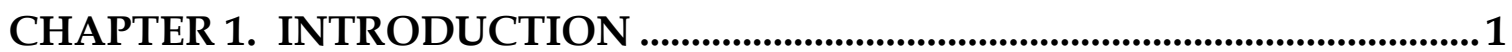

CHAPTER 2. REVIEW OF THE LITERATURE ........................................................

Factors That Influence Posttreatment Stability ......................................................... 3

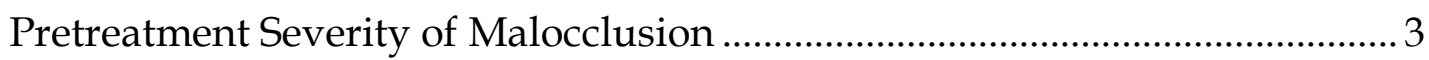

Tooth Positions Following Treatment ............................................................. 3

Original Malocclusion Type...........................................................................

Changes in Mandibular Intercanine Width....................................................... 4

Bone Morphology in the Mandibular Incisor Area............................................... 4

Changes in Arch Characteristics .................................................................... 5

Influences from the Integument .................................................................... 5

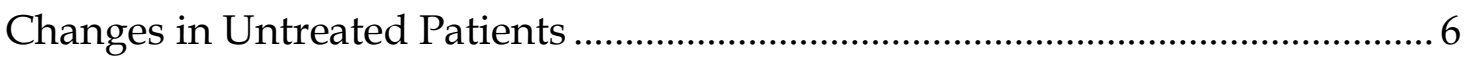

Dental Changes in Untreated Normal Occlusions ..............................................

Skeletal Changes in Untreated Normal Occlusions ............................................11

Soft Tissue Profile Changes in Untreated Normal Occlusions ......................... 17

Long Term Posttreatment Changes ..................................................................19

Skeletal Changes Following Treatment .......................................................... 19

Dental Changes Following Treatment …………………………………...... 21

Soft Tissue Profile Changes Following Treatment .............................................. 24

CHAPTER 3. MATERIALS AND METHODS .......................................................26

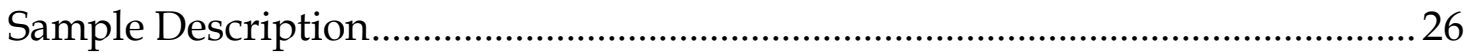

Cephalometric Analysis................................................................................. 26

Statistical Analysis ................................................................................................ 39

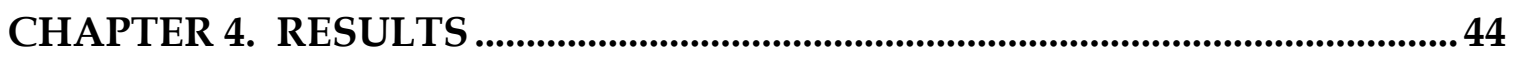

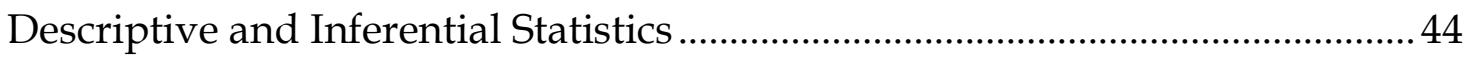

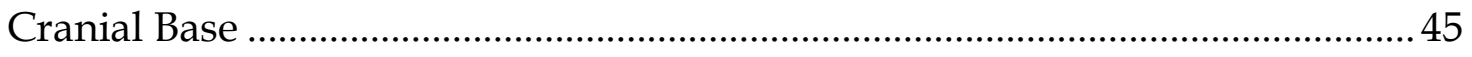

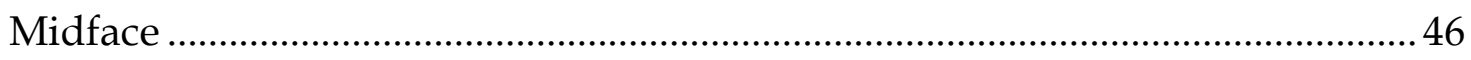

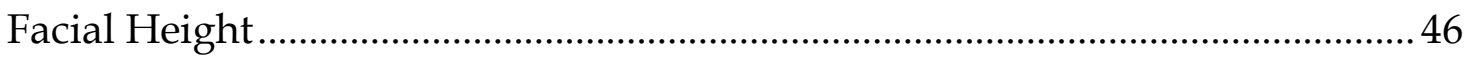

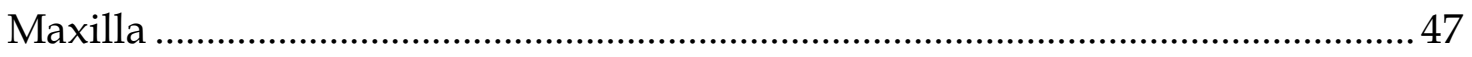

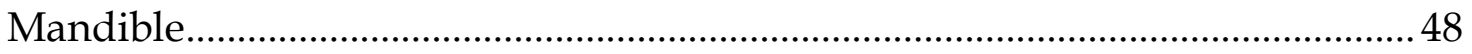

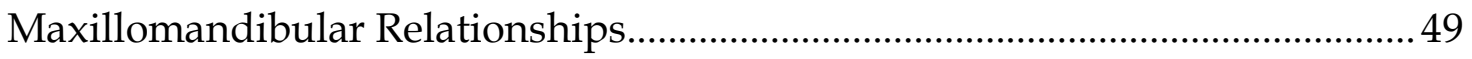

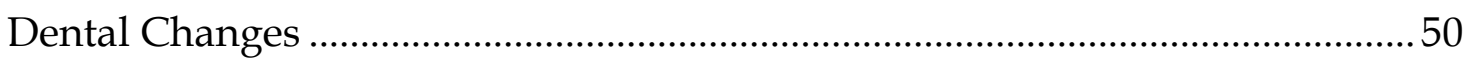




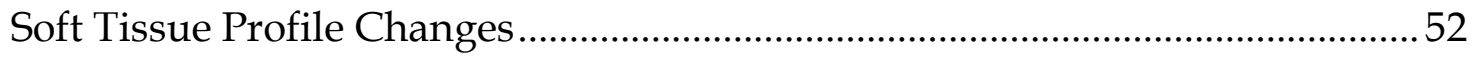

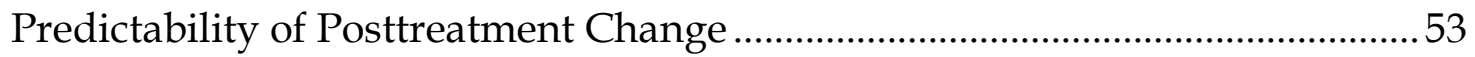

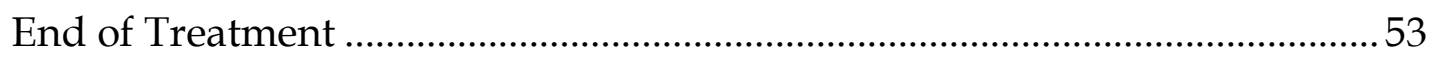

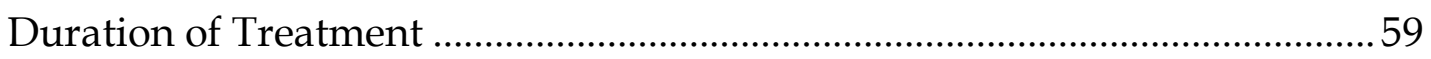

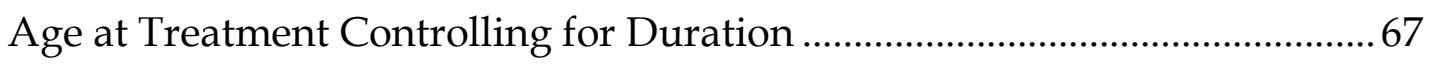

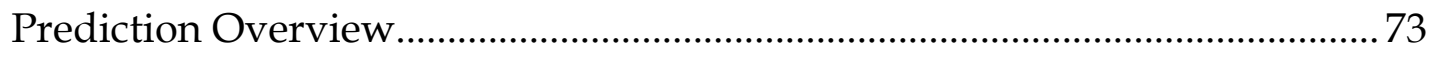

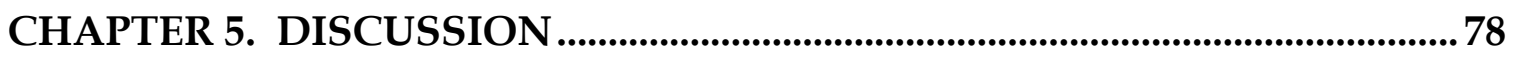

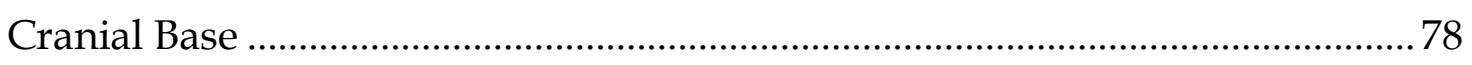

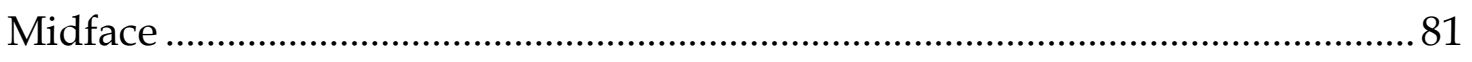

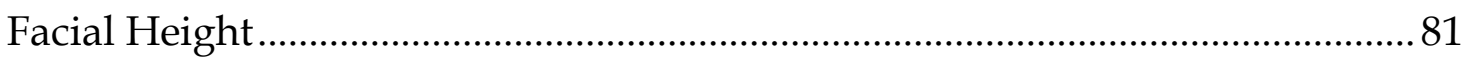

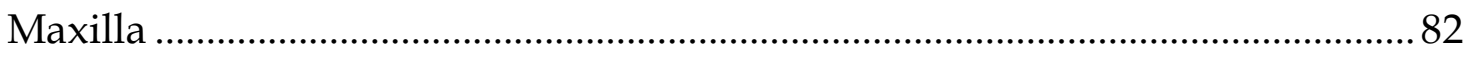

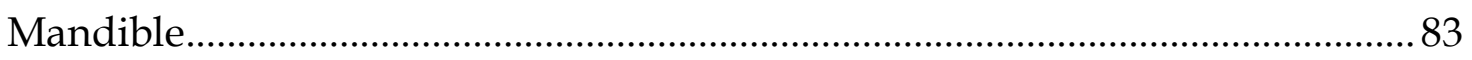

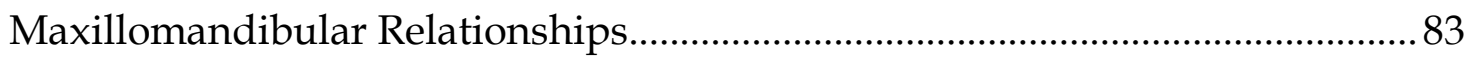

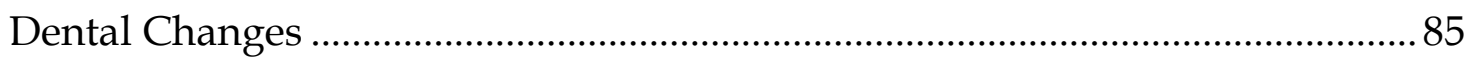

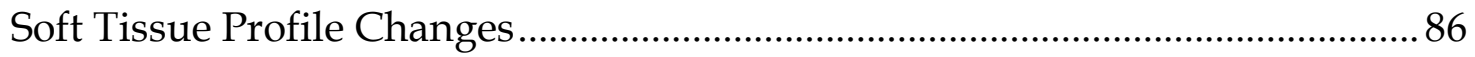

CHAPTER 6. SUMMARY AND CONCLUSIONS..................................................

LIST OF REFERENCES ...................................................................................................91

APPENDIX A. DESCRIPTIVE STATISTICS AND TESTS FOR

POSTTREATMENT CHANGES ....................................................................................97

APPENDIX B. RESULTS OF MIXED-MODEL ANOVA TESTING FOR

POSTTREATMENT CHANGES ..........................................................................127

APPENDIX C. GRAPHICAL REPRESENTATIONS OF THE

DISTRIBUTIONS OF POST-TREATMENT CHANGE FOR EACH

CEPHALOMETRIC VARIABLE .........................................................................132

APPENDIX D. GRAPHICAL REPRESENTATIONS OF

CEPHALOMETRIC VARIABLES ...............................................................................161

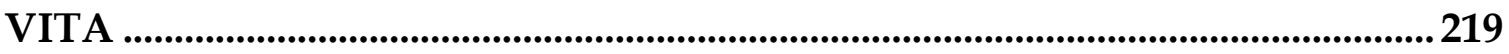




\section{LIST OF TABLES}

Table 2-1. Descriptive statistics for pooled (males and females) cast measurements at T1 (median age $=9$ years), $\mathrm{T} 2$ (median age $=13$ years), and T3 (median age $=20$ years).

Table 2-2. Changes in cephalometric dental parameters at T1 (median age $=9$ years), $\mathrm{T} 2$ (median age $=13$ years), and T3 (median age $=20$ years)

Table 2-3. Comparison of dental changes for untreated subjects (T1-T2) ........12

Table 2-4. Changes in cephalometric values at $\mathrm{T} 1$ (median age $=9$ years), $\mathrm{T} 2$ (median age $=13$ years), and T3 (median age $=20$ years) ..........13

Table 2-5. Comparison of skeletal changes for untreated subjects (T1-T2)...... 16

Table 2-6. Mean changes in cephalometric values ............................................. 20

Table 2-7. Mean posttreatment changes in dental measurements...................... 22

Table 3-1. Cephalometric measurement abbreviations and corresponding measurement descriptions

Table 4-1. Results of linear regression analysis testing whether patient's age at end of treatment is predictive of the amount of posttreatment change

Table 4-2. Results of linear regression analysis testing whether the duration of time following treatment until the recall examination is predictive of the amount of post-treatment change

Table 4-3. Results of analysis of covariance testing for a statistical dependence between the subject's age at the end of treatment and the amount of posttreatment change with the duration of the recall examination as the covariate.....

Table 4-4. Results of ANCOVA for nasion-perpendicular to A point ................71

Table 4-5. Results of ANCOVA for nasion-perpendicular to A point ............... 72

Table 4-6. Results of ANCOVA for U1 to $\mathrm{NA}^{\mathrm{o}}$.................................................. 74

Table 4-7. Results of ANCOVA for L6 to $\mathrm{W}$ point ................................................ 75

Table 4-8. Results of ANCOVA for nasolabial angle ............................................76 


\section{LIST OF FIGURES}

Figure 3-1. Ages of subjects at the posttreatment and recall examinations.......27

Figure 3-2. Cephalometric skeletal and soft-tissue landmarks ............................ 29

Figure 3-3. Cephalometric dental landmarks.......................................................... 30

Figure 4-1. Plot of the subject's age at the end of treatment against the amount of posttreatment change in NaPerp to A point .....................57

Figure 4-2. Plot of the subject's age at the end of treatment against the amount of posttreatment change in NaPerp to B point... .57

Figure 4-3. Plot of the subject's age at the end of treatment against the amount of posttreatment change in distal L6 to $\mathrm{W}$ point. .58

Figure 4-4. Plot of the subject's age at the end of treatment against the amount of posttreatment change in nasolabial angle.

Figure 4-5. Plot of the subject's duration to the recall examination against the amount of posttreatment change (increase) in Sella-Nasion distance.

Figure 4-6. Plot of the subject's duration to the recall examination against the amount of posttreatment change in Sella-A distance...

Figure 4-7. Plot of the subject's duration to the recall examination against the amount of posttreatment change in L1-NB angle

Figure 4-8. Plot of the subject's duration to the recall examination against the amount of posttreatment change in IMPA

Figure 4-9. Plot of the subject's duration to the recall examination against the amount of posttreatment change in FMIA .66

Figure 4-10. Plot of the subject's duration to the recall examination against the amount of posttreatment change in overbite

Figure 4-11. Association between the subject's age at the end of treatment against the amount of posttreatment change in nasionperpendicular to A point

Figure 4-12. Association between the subject's age at the end of treatment against the amount of posttreatment change in the angle U1 to Sella-Nasion

Figure 4-13. Association between the subject's age at the end of treatment against the amount of posttreatment change in the angle $\mathrm{U} 1$ to NA 
Figure 4-14. Association between the subject's age at the end of treatment against the amount of posttreatment change in the distance L6

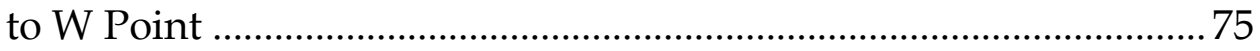

Figure 4-15. Association between the subject's age at the end of treatment against the amount of posttreatment change in the nasolabial

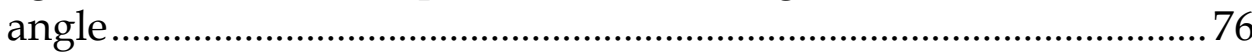

Figure 5-1. Plot of the amount of growth following treatment in the posterior cranial base against the age at the start of treatment .......80 


\section{CHAPTER 1. INTRODUCTION}

Achieving posttreatment stability of orthodontic treatment has proven to be one of the most challenging issues in all of orthodontics, and it is a concern that the specialty will need to continue to address in the future. Classic orthodontic literature contains an abundance of insight into the struggles that orthodontists have encountered with relapse, and yet many of these problems are as prevalent today as they were in the early years of the specialty. Alvin Oppenheim (1934) stated that posttreatment relapse was the most difficult problem to be faced in orthodontics.

Orthodontic treatment has been used not only to correct malpositions of the teeth within the dental arches but also to induce craniofacial changes through the use of various treatment mechanics. Although the changes in the craniofacial skeleton induced by orthodontic treatment are believed to improve functional relationships, they may not have the same inherent stability as those that develop naturally. Because posttreatment craniofacial positions and relationships following orthodontic treatment are not the natural skeletal relationships that are developed from a normal, unaltered growth process, there is potential for relapse (Ormiston et al. 2005).

Over the past 50 years, there have been numerous articles published on the posttreatment change experienced post-adolescence and into adulthood. A major source of information on long-term posttreatment stability has been from the graduate orthodontic program at the University of Washington. Their research efforts have shown that significant posttreatment relapse does occur over the long term, but the measurement of relapse itself has been complicated by the growth and maturational changes within the craniofacial complex that occur concurrently. Many would argue that the studies out of the University of Washington may not provide an accurate depiction of the posttreatment stability to be expected from an experienced orthodontists in private practice, since these were university studies that involved patients who were treated under different circumstances than one might find in private practice. While the results from the Washington studies have not been favorable, there is research that suggests that there is no significant difference in the overall quality of orthodontic treatment outcomes between patients treated in university programs and private practices (Cook et al. 2005).

The two-fold purpose of the present study is to describe the posttreatment changes that resulted from non-extraction orthodontic therapy by a single, experienced orthodontist in private practice, and secondly, to evaluate whether these findings are congruent with those out of the University of Washington and 
others that suggest that much of orthodontic correction is lost over the long-term. Recall records were taken from the people in the study at an average of 12.5 years post-treatment. Statistical analysis of the cephalometric variables was used to analyze the posttreatment changes. 


\title{
CHAPTER 2. REVIEW OF THE LITERATURE
}

\author{
Factors That Influence Posttreatment Stability
}

\section{Pretreatment Severity of Malocclusion}

A well known predictor of orthodontic relapse is the pre-treatment severity of the malocclusion. Previous studies suggest that the greater the severity of the initial malocclusion, the greater the amount of post-treatment relapse that can be expected (Little, Riedel and Årtun 1988; Ormiston et al. 2005). According to a 10 year recall study by Al Yami et al. (1999), an average of $67 \%$ of the achieved orthodontic treatment result (as measured by the overall reduction in PAR index score) was maintained at 10 years posttreatment for all severities of initial malocclusion. These findings suggest that the treatment results of patients with more severe index scores before treatment tended to deteriorate more, although the reductions in their PAR scores decreased by the same mean percentage at long-term posttreatment than did less severe initial malocclusions. To clarify, PAR scores increased for all patients by an average of $33 \%$ at recall, but since the cases with greater severity of malocclusions presented with greater PAR scores initially, the total amount of relapse accounted for by the $33 \%$ increase in those cases was greater overall than that seen in cases with lesser initial PAR scores. This mirrors the findings of a more recent study out of the University of Washington (Ormiston et al. 2005) that also found the initial severity of malocclusion, as graded by the PAR index, to be negatively correlated with posttreatment stability.

\section{Tooth Positions Following Treatment}

Edward Angle has been quoted (Weinberger 1926: 13) as saying "the best balance, the best harmony, the best proportions of the mouth in its relation to the other features require that there shall be a full complement of teeth, and that each tooth shall be made to occupy its normal position - i.e., normal occlusion." The importance of a normal occlusion in achieving balance and harmony can also be applied to the influence of the quality of posttreatment occlusion on stability.

Reitan (1969) agreed that the stability of tooth position depends largely on how well a case has been treated. He suggested that uprighting teeth over basal bone and inducing bodily movements (i.e., translation) rather than tipping movements to correct tooth positions can appreciably reduce the likelihood of tooth migration following treatment. He also suggested that since a slight 
change towards the original position is likely to occur after treatment, overcorrection of tooth position should be incorporated into treatment to compensate for relapse.

\section{Original Malocclusion Type}

Elements of the original malocclusion can influence the amount of orthodontic relapse. For example, research suggests that patients who receive treatment for anterior open bite can expect a greater amount of relapse in their overbite correction than patients with other types of malocclusions (Little et al. 1981; Shields et al. 1985). Both of these studies evaluated first-premolar extraction cases treated by traditional edgewise orthodontics. According to these studies, an average of 50 to 70 percent of overbite correction is lost due to relapse. Bresonis and Grewe (1974) found that Class II division 1 malocclusions experienced about twice as much relapse in overjet correction as Class II division 2 malocclusions at five years posttreatment.

\section{Changes in Mandibular Intercanine Width}

It is widely reported that any significant change in mandibular intercanine width from the pretreatment width is likely to relapse towards its original dimension (Blake and Bibby 1998). For this reason, clinicians tend to consider mandibular intercanine width as an inviolable dimension if posttreatment stability of orthodontic treatment is to be maintained. Burke et al. (1998) found that mandibular intercanine width was expanded during treatment by an average of $0.8 \mathrm{~mm}$ in Class II division 1 non-extraction patients and $2.0 \mathrm{~mm}$ in Class II division 2 patients. They also found that mandibular intercanine width experiences an average constriction of $1.2 \mathrm{~mm}$ in Class II division 1 nonextraction patients and $1.6 \mathrm{~mm}$ in Class II division 2 patients at 2 years posttreatment. According to Udhe, Sadowsky and BeGole (1983), mandibular intercanine width reduction following removal of retention showed a strong positive correlation with late incisor crowding. These findings and those from others indicate that changes in mandibular intercanine width during treatment are a major causative factor in orthodontic relapse.

\section{Bone Morphology in the Mandibular Incisor Area}

Crowding of the mandibular incisors is a primary concern when it comes to posttreatment relapse, and there has been considerable research on the posttreatment stability of these teeth. Rothe et al. (2006) suggested that the 
amount or structure of the mandibular bone surrounding these teeth is a potential risk factor. Through the measurement of the thickness of mandibular cortical bone on panoramic and cephalometric radiographs, it was concluded that patients with thinner mandibular cortical bone around these teeth are at increased risk for incisor relapse following orthodontic treatment. It was also concluded from a fractal analysis of periapical radiographs that the trabecular bone structure around these teeth had no correlation with incisor relapse.

\section{Changes in Arch Characteristics}

It has been suggested that changes in arch form and arch width due to treatment are unstable. These two dental arch characteristics tend to return toward their pretreatment values after treatment, so a patient's pretreatment arch form and arch width appear to be important factors in the future stability of the dentition. Research by De La Cruz et al. (1995) found that there is a rounding of the arch form during treatment followed by a change to a more tapered arch form at 10 years posttreatment. They also found that arch widths, as measured in the canine, premolar, and first molar regions, almost always showed constriction at long-term recall examinations due to the tendency to expand the arches during treatment.

It has also been suggested that increases in maxillary or mandibular arch length during orthodontic treatment are largely unstable. For the most part, this has been accomplished through the distal movement of posterior teeth with various distalization appliances. Hays Nance (1947: 254) was once quoted as saying this regarding the distal movement of posterior teeth: "Such measures might be defensible were it not for the abundant clinical proof to prove that these teeth moved distally will not stay distally, but will come forward again, as if seeking to remain in the positions from which they were disturbed." Current research supports this statement by Nance, especially with respect to increases in mandibular arch length. Increases in maxillary arch length through distalization have been accomplished with more long-term success, but the current optimism on the long-term stability that can be achieved in the maxilla may be clouded by the relative lack of stability that has been attainable in the mandible.

\section{Influences from the Integument}

Function of the lips as well as that of the facial and mandibular muscles can influence tooth alignment and stability. Pepicelli, Woods and Briggs (2005) suggested that a patient's facial form is a consideration in determining the most stable incisal positions and angulations at the end of treatment. They found that 
protrusive incisors were more stable over the long term in people with shorter facial types (i.e., brachyfacial patterns) and stronger musculature than in people with longer facial types (i.e., dolichofacial patterns) and weaker musculature. They suggested that the differences in biting forces produced by these two types of facial patterns could be the underlying reason for the differences in incisal stability.

Reitan (1969) suggested that an oversized tongue or improper tongue position commonly results in Class III malocclusions with an enlarged lower dental arch. The lingual pressure being exerted by the tongue onto the mandibular teeth often results in posterior crossbites, and this pressure also creates a potential for relapse of transverse corrections in tooth position following treatment. In these types of cases, some form of retention in the mandibular arch is necessary shortly after treatment in order to control posttreatment contraction of stretched fibrous tissue and to resist the pressure caused by muscle function. It is supposed that, as new bone calcifies around the mandibular posterior teeth during the retention period, the new positions of the alveoli will act as solid blocks that will prevent the tongue from assuming its former position.

\section{Changes in Untreated Patients}

Harry Israel (1968) performed an extensive study detailing longitudinal craniofacial growth changes occurring beyond the second decade of life. He found that bony enlargement occurred in the majority of the 50 cranial measurements that he assessed. The overall size of the mandible itself was reported to enlarge by five to seven percent over a 30 year period. While he admitted that this "growth" in no way compares to that found in the younger years, it exists nonetheless.

Behrents (1985) reinforced and extended the findings of Israel when he described craniofacial growth changes that occur during adulthood. He found too that craniofacial growth does not stop in young adulthood but is a continuous process even into late adult life. Behrents reported that people experience significant "differential growth" within the craniofacial complex throughout adulthood due to increases in the size of their skeletal structures and to changes in their shape.

Similar to changes in the skeleton that occur with normal aging, dental and soft tissue changes are continuous and significant beyond the second decade of life. Soft tissue changes to be expected are partially associated with reductions in muscle mass and subcutaneous fat within the face that occur. Reduction in 
soft tissue volumes in the face are, on average, more pronounced in females than in males (Meema et al. 1973). Since human teeth undergo maturational changes and are subject to degradation due to attrition, caries, trauma, and periodontal involvement, there are also cephalometrically quantifiable changes in the dentition that occur with normal aging.

It is important to note that any craniofacial changes occurring due to posttreatment relapse take place simultaneously with changes that occur as a result of normal growth processes.

\section{Dental Changes in Untreated Normal Occlusions}

Studies have consistently shown that lower incisor crowding increases with age in untreated, normal occlusions, and this increase is shown to be continuous into late adulthood (Eslambolchi et al. 2008). Most studies have also shown that crowding increases primarily during early adulthood, and although individual differences in the amount of crowding are multifactorial, the primary determinants are unidentified (Buschang and Shulman 2003).

Sinclair and Little (1983) detailed the maturation of untreated, normal occlusions from adolescence to early adulthood by examining the dental casts of 65 individuals ( 33 boys and 32 girls) in the mixed dentition (9 to 10 years), early permanent dentition (12 to 13 years), and early adulthood (19 to 20 years). Dental arch length showed consistent decreases with age, decreasing by an average of $2.9 \mathrm{~mm}$ during the first interval, and decreasing by an average of 2.0 $\mathrm{mm}$ during the second interval. All arch widths, including intercanine width, interpremolar width, and intermolar width, exhibited significant decreases during both intervals. Overjet increased by an average of $0.4 \mathrm{~mm}$ in the first interval but decreased by approximately the same amount during the second interval, making the overall change insignificant. Overbite also increased by an average of $0.4 \mathrm{~mm}$ in the first interval, but unlike overjet, it decreased by $0.6 \mathrm{~mm}$ during the second interval, making the overall change significant towards a reduction in overbite by the final recall. Incisor irregularity showed no statistically significant change during the first interval, but a statistically significant $(\mathrm{P}<0.001)$ increase in incisor irregularity of $0.7 \mathrm{~mm}$ occurred during the second interval. The above results are shown in greater detail in Table 2-1.

Sinclair and Little (1985) also studied the cephalometric head films of the same sample used in their earlier cast study. Their findings included both skeletal changes (which will be detailed in the next section) and dental changes. Of the 25 cephalometric measurements recorded for the participants in this 
Table 2-1. Descriptive statistics for pooled (males and females) cast measurements at T1 (median age $=9$ years), $\mathrm{T} 2$ (median age $=13$ years), and T3 (median age $=20$ years).

\begin{tabular}{|c|c|c|c|c|c|c|}
\hline \multirow[b]{2}{*}{ Measurements } & \multicolumn{2}{|c|}{$\mathrm{T} 1$} & \multicolumn{2}{|c|}{$\mathrm{T} 2$} & \multicolumn{2}{|c|}{ T3 } \\
\hline & Mean & SD & Mean & SD & Mean & SD \\
\hline Arch length & 63.12 & 2.98 & $60.24+$ & 3.41 & $58.29+$ & 3.15 \\
\hline Intercanine width & 25.45 & 1.47 & 25.14 & 1.43 & $24.70+$ & 1.53 \\
\hline Intermolar width & 43.74 & 2.40 & 43.69 & 2.77 & 43.59 & 3.16 \\
\hline Overjet & 2.87 & 1.00 & $3.31+$ & 1.21 & $2.82+$ & 1.10 \\
\hline Overbite & 2.95 & 1.20 & $3.35+$ & 1.00 & $2.76+$ & 1.20 \\
\hline Incisor irregularity & 2.22 & 1.23 & 2.00 & 1.17 & $2.70+$ & 1.64 \\
\hline
\end{tabular}

Notes: All variables are in millimeters (mm). SD, standard deviation. + Statistically significant difference $(\mathrm{P}<0.05)$ from the previous measurement.

Source: Sinclair PM, Little RM. Maturation of untreated normal occlusions. Am J Orthod Dentofacial Orthop 1983;83:114-23. 
study, 11 were used for quantifying dental changes, and these results are shown in Table 2-2.

Their results showed an incredible degree of stability in incisor angulation. This was particularly true for the upper incisors, which showed no statistically significant change at any stage. The lower incisors tended to show a small increased proclination in both sexes, as the angulation of the lower incisor to NB and the angulation of the lower incisor to mandibular plane both showed small, but significant increases at both intervals. It should be noted, however, that over the total observation period, from $\mathrm{T} 1$ to $\mathrm{T} 3$, none of these changes was of statistical significance $(\mathrm{P}>0.05)$. Interincisal angle also showed relatively good stability, as the only statistically significant change was an increase from T2 to T3 in males. The changes in interincisal angle, however, were not statistically significant in either sex over the total observation period from T1 to T3.

Through mandibular cephalometric superimposition, which shows changes in the dentition relative to the skeletal base, some additional information was revealed. A statistically significant increase in lower incisor proclination was found in males from T2 to T3. This change was closely correlated with the direction of mandibular growth and with the degree of forward movement of the mandibular incisors. Also, the mandibular superimposition showed a statistically significant overall degree of forward movement of the mandibular incisor in males over the total observation period from T1 to T3. These changes were closely correlated with the degree of forward movement of the mandibular molar and with changes in incisor angulation.

There was significant maxillary molar eruption relative to the palatal plane in both sexes and across intervals. These changes were associated with the degree of posterior facial growth and the increase in upper facial height. There was also a significant amount of mandibular molar eruption from T1 to T3, with the greatest amount occurring between $\mathrm{T} 2$ and $\mathrm{T} 3$ in both sexes. These changes were closely associated with the degree of posterior facial growth and the increase in lower facial height. Mandibular superimpositions revealed a statistically significant mesial tipping of the maxillary first molar relative to the X-axis (horizontal plane) throughout the T1 to T3 period. Similarly, the mandibular first molar exhibited a significant mesial tipping relative to the Y-axis (vertical plane) throughout the total observation period, accompanied by continued mesial movement of the molar.

Driscoll-Gilliland, Buschang, and Behrents (2001) evaluated the growth and stability of 44 untreated subjects from the Broadbent-Bolton Growth Study by examining the dental casts and lateral cephalograms at the beginning of the observation period (14.3 \pm 1.5 years) and at long term recall (23.2 \pm 3.4 years). 
Table 2-2. Changes in cephalometric dental parameters at T1 (median age $=9$ years), $\mathrm{T} 2$ (median age $=13$ years), and T3 (median age $=20$ years).

\begin{tabular}{|c|c|c|c|c|c|c|}
\hline \multirow[b]{2}{*}{ Variables } & \multicolumn{2}{|c|}{$\mathrm{T} 1$ to $\mathrm{T} 2$} & \multicolumn{2}{|c|}{$\mathrm{T} 2$ to $\mathrm{T} 3$} & \multicolumn{2}{|c|}{$\mathrm{T} 1$ to $\mathrm{T} 3$} \\
\hline & Males & Females & Males & Females & Males & Females \\
\hline L1-NB (mm) & 0.75 & 0.10 & $-0.35+$ & $0.48^{*}+$ & 0.40 & 0.58 \\
\hline L1-NB $\left(^{\circ}\right)$ & -0.67 & -0.24 & $-2.96^{*}+$ & $1.17+$ & -3.63 & 0.93 \\
\hline L1-MP $\left(^{\circ}\right)$ & $4.84^{*}+$ & $-0.34+$ & $-1.43^{*}+$ & $2.39^{*}+$ & 3.41 & 2.05 \\
\hline $\mathrm{U} 1-\mathrm{L} 1\left(^{\circ}\right)^{\prime}$ & -2.10 & 1.20 & $4.05^{*}+$ & $-1.29+$ & 1.95 & -0.09 \\
\hline U1-NA $\left(^{\circ}\right)$ & 4.83 & -0.52 & -5.65 & 0.50 & -0.82 & -0.02 \\
\hline U1-NA (mm) & 0.45 & 0.37 & -0.01 & 0.43 & 0.44 & 0.80 \\
\hline U1-SN $\left(^{\circ}\right)$ & 0.03 & -0.48 & 0.48 & 0.86 & 0.51 & 0.38 \\
\hline ANS-L1 (mm) & $1.30^{*}$ & $1.48^{*}$ & $1.80^{*}+$ & $0.78+$ & $3.10^{*}$ & $2.26^{*}$ \\
\hline U1-Me (mm) & $3.36^{*}+$ & $1.37+$ & $3.69^{*}+$ & $1.56^{*}+$ & $7.05^{*}+$ & $2.93^{*}+$ \\
\hline U6-PP (mm) & $3.38^{*}$ & $4.96^{*}$ & $4.42^{*}+$ & $1.63^{*+}$ & $7.80^{*}$ & $6.59^{*}$ \\
\hline L6-MP (mm) & $1.91^{*}$ & -0.22 & $3.94^{*}+$ & $1.85^{*}+$ & $5.85^{*}+$ & $1.63+$ \\
\hline
\end{tabular}

Notes: *Statistically significant change $(\mathrm{P}<0.05)$. +Statistically significant difference $(\mathrm{P}<0.05)$ between males and females.

Source: Sinclair PM, Little RM. Dentofacial maturation of untreated normals. Am J Orthod 1985;88:146-56. 
They found that most dental characteristics changed significantly over the long term observation period. The largest observed change was the 4 to $5 \mathrm{~mm}$ inferior displacement of the lower incisor relative to Sella-Nasion attributable to growth. Since the lower incisor erupted approximately $1.5 \mathrm{~mm}$ there was a net downward movement of the lower incisor relative to Sella. The anterior displacement of the lower incisor attributable to growth was approximately 2 $\mathrm{mm}$. Overjet did not show a statistically significant change, while overbite showed a small, but significant $(\mathrm{P}<0.05)$ decrease of $0.3 \mathrm{~mm}$. The upper incisor moved forward relative to Sella approximately $1.6 \mathrm{~mm}$. Contact irregularity increased by $0.9 \mathrm{~mm}$, while space irregularity increased by $1.3 \mathrm{~mm}$. Both of these long term changes were of high statistical significance $(\mathrm{P}<0.001)$. Arch length also decreased significantly by $1.2 \mathrm{~mm}$. The results from this study can be seen in greater detail in Table 2-3.

Harris and Behrents (1988) assessed sagittal changes in the molar relationship of untreated individuals with full dentitions who were followed longitudinally from young adulthood (approximately 20 years of age on average) to older adulthood (approximately 55 years). They found that the Class I molar relationship was the most stable, as none of the 69 cases starting in a Class I molar relationship moved from that position. Class II and Class III molar relationships, in contrast, became significantly more Class II (i.e., a greater distocclusion) and Class III (i.e., a greater mesiocclusion), respectively. The Class II cases showed an average $0.8 \mathrm{~mm}$ increase in the severity of the Class II relationship, with 29 of the 30 cases staying the same or getting worse over time. Class III cases showed an average $1.2 \mathrm{~mm}$ increase in the severity of the Class III relationship, with 12 of the 13 cases staying the same or getting worse.

These findings suggest that a Class I molar relationship is intrinsically the most stable molar relationship in natural, untreated occlusions. The finding that Class II molar relationships tend to become more Class II over time in untreated occlusions is of particular importance to the present study, since this tendency could, in concept, influence the stability of treated Class II cases.

\section{Skeletal Changes in Untreated Normal Occlusions}

Sinclair and Little (1985) recorded 14 cephalometric measurements that were used to quantify the skeletal changes in the untreated sample detailed in the previous section. These results are shown in Table 2-4. Their results showed that changes in SNA angle were relatively small, with only males showing statistically significant increases from T1-T2 and from T2-T3. The SNB angle increased significantly for the entire T1-T3 period in both sexes, with the changes 
Table 2-3. Comparison of dental changes for untreated subjects (T1-T2).

\begin{tabular}{lccc}
\hline \multicolumn{1}{c}{ Variable } & Mean & SD & Sig \\
\hline L1 tip eruption & 1.5 & 1.4 & $* *$ \\
L1 tip growth vertical & 5.3 & 3.6 & $* *$ \\
L1 tip growth horizontal & 1.7 & 2.8 & $* *$ \\
L1 apex eruption & 1.4 & 1.4 & $* *$ \\
L1 apex growth vertical & 5.3 & 3.6 & $* *$ \\
L1 apex growth horizontal & 2.0 & 3.4 & $* *$ \\
U1 tip total horizontal & 1.6 & 2.0 & $* *$ \\
U1 apex total horizontal & 1.9 & 2.0 & $* *$ \\
U1/SN & 0.2 & 3.6 & NS \\
U1/L1 & 1.4 & 5.0 & NS \\
Overjet & -0.2 & 1.0 & NS \\
Overbite & -0.3 & 0.8 & $*$ \\
Arch length & -1.2 & 1.0 & $* *$ \\
Contact irregularity & 0.9 & 1.4 & $* *$ \\
Space irregularity & 1.3 & 1.3 & $* *$ \\
\hline
\end{tabular}

Notes: All variables are in millimeters (mm). SD, standard deviation. Sig, significance. NS, not statistically significant. ${ }^{*} \mathrm{P}<0.05 .{ }^{* *} \mathrm{P}<0.001$.

Source: Driscoll-Gilliland J, Buschang MA, Behrents RG. An evaluation of growth and stability in untreated and treated subjects. Am J Orthod Dentofacial Orthop 2001;120:588-97. 
Table 2-4. Changes in cephalometric values at $\mathrm{T} 1$ (median age $=9$ years), $\mathrm{T} 2$ ( median age $=13$ years), and T3 (median age $=20$ years).

\begin{tabular}{|c|c|c|c|c|c|c|}
\hline \multirow[b]{2}{*}{ Variables } & \multicolumn{2}{|c|}{$\mathrm{T} 1$ to $\mathrm{T} 2$} & \multicolumn{2}{|c|}{$\mathrm{T} 2$ to $\mathrm{T} 3$} & \multicolumn{2}{|c|}{$\mathrm{T} 1$ to $\mathrm{T} 3$} \\
\hline & Males & Females & Males & Females & Males & Females \\
\hline SNA $\left({ }^{\circ}\right)$ & $0.66^{*}$ & $0.04^{*}$ & $0.67^{*}$ & 0.35 & $1.34^{*}$ & 0.39 \\
\hline $\operatorname{SNB}\left({ }^{\circ}\right)$ & $0.80^{*}$ & 0.49 & $1.47^{*}$ & $0.73^{*}$ & $2.27^{*}$ & $1.22 *$ \\
\hline $\mathrm{ANB}\left({ }^{\circ}\right)$ & -0.14 & -0.44 & -0.79 & -0.38 & -0.93 & $-0.82^{*}$ \\
\hline MP-SN $\left(^{\circ}\right)$ & $-0.86^{*}$ & -0.38 & -3.00 & $-1.96^{*}$ & $-3.86^{*}$ & $-2.34^{*}$ \\
\hline $\mathrm{Y}$ axis $\left({ }^{\circ}\right)$ & 0.06 & -0.12 & $-1.11^{*}$ & -0.35 & $-1.05^{*}$ & -0.47 \\
\hline Gonial angle $\left(^{\circ}\right)$ & $-1.29^{*}$ & 1.14 & $-2.90^{*}$ & $-3.32^{*}$ & $-4.19^{*}$ & $-2.18^{*}$ \\
\hline S-N (mm) & $2.62^{*}$ & $1.62^{*}$ & $4.26^{*}+$ & $1.64^{*}+$ & $6.88^{*}+$ & $3.26^{*}+$ \\
\hline $\begin{array}{l}\text { Cranial base } \\
\text { angle }\left(^{\circ}\right)\end{array}$ & 0.04 & $1.45^{*}$ & $1.29^{*}+$ & $0.98^{*}$ & $1.33^{*}$ & $2.43^{*}$ \\
\hline Se-Go (mm) & $6.23^{*}$ & 3.33 & $10.26^{*}+$ & $5.16^{*}+$ & $16.49^{*}+$ & $8.49^{*}+$ \\
\hline Ar-Go (mm) & $3.47^{*}$ & 1.34 & $7.96^{*}+$ & $3.65^{*}+$ & $11.43^{*+}$ & $4.99 *+$ \\
\hline $\mathrm{Na}-\mathrm{Me}(\mathrm{mm})$ & $7.92^{*}$ & $4.52^{*}$ & $10.10^{*}+$ & $4.71^{*}+$ & $18.12^{*}+$ & $9.23^{*}+$ \\
\hline Na-ANS (mm) & $4.30^{*}+$ & $2.14^{*}+$ & $2.90^{*}$ & $2.01^{*}$ & $7.20^{*}+$ & $4.15^{*}+$ \\
\hline ANS-Me (mm) & $3.52^{*}$ & $2.21^{*}$ & $6.67^{*+}$ & $2.30^{*}+$ & $10.19^{*}+$ & $4.51^{*}+$ \\
\hline
\end{tabular}

Notes: *Statistically significant change $(\mathrm{P}<0.05)$. +Statistically significant difference $(\mathrm{P}<0.05)$ between males and females.

Source: Sinclair PM, Little RM. Dentofacial maturation of untreated normals. Am J Orthod 1985;88:146-56. 
being more noticeable in males. The amount of forward growth in the maxilla as described by the SNA angle from T2-T3 was associated with the degree of forward movement of the mandible as described by the SNB angle in both sexes. Overall, the mandible grew approximately twice as much as the maxilla from $\mathrm{T} 1-\mathrm{T} 3$ in males and approximately three times as much as the maxilla in females during the same observation period. The resultant ANB angle showed a small but statistically significant decrease from T1-T3 for the group as a whole.

Forward (bite-closing) rotation of the mandible was shown in both sexes by a statistically significant and continuous decrease in the MP-SN angle from T1-T3, with most of this change occurring from T2-T3. This change was closely correlated with the increase seen in SNB angle, which suggested an association with late mandibular growth. There was also an association between the amounts of maxillary and mandibular molar eruption.

Throughout the entire T1-T3 time period, the length of the anterior cranial base (Se-Na) increased significantly in both sexes, with males showing more than twice as much growth in this dimension than females. The cranial base angle (CBR-Se-Ba) also showed a statistically significant overall increase, with the majority of the change occurring from T2-T3.

Upper anterior facial height (Na-ANS) showed a significant increase throughout the T1-T3 time interval, with the rate of increase slowing from T2-T3. Males showed nearly double the amount of overall increase in this dimension as females. Lower anterior facial height (ANS-Me) also showed a significant increase throughout the T1-T3 time interval, with males showing more than double the amount of overall increase as in females. Total anterior facial height (Na-Me) increased by an average of $18 \mathrm{~mm}$ in males and $9 \mathrm{~mm}$ in females over the total observation period (T1-T3).

Posterior facial height (Se-Go, Ar-Go) also showed a significant increase throughout the T1-T3 time period, with the majority of this increase occurring from T2-T3. This change was associated with the vertical growth of the anterior face and the amount of eruption of the mandibular first molar.

Mandibular superimpositions revealed that there was a change to a more vertical growth direction over the entire T1-T3 time period. This change was more pronounced in males from $\mathrm{T} 2-\mathrm{T} 3$ and resulted in a significant overall difference between the sexes in growth direction. The amount of molar eruption was closely correlated with the change in growth direction.

In summary, Sinclair and Little's findings showed the importance of maxillary development, particularly in the vertical plane, as it was associated 
with the amount and direction of mandibular growth. Late mandibular growth was found to be a counter-clockwise (bite-closing) rotation of the mandible that occurred after the cessation of vertical maxillary growth. The degree of forward (bite-closing) mandibular rotation was associated with the increase in SNB angle and the amount of both maxillary and mandibular molar eruption.

A longitudinal study by Ochoa and Nanda (2004) compared maxillary and mandibular growth from 6 to 20 years of age. The sample comprised 28 untreated subjects (15 female, 13 male), all of whom had Class I malocclusions. Their findings confirmed some of the findings of Sinclair and Little, as they also found that mandibular length (Ar-Pg) increased more than twice as much as maxillary length (ANS-PNS) from adolescence (mean age $=12$ years) into early adulthood (mean age $=20$ years). The females had less incremental growth and shorter duration of mandibular growth, so that their facial skeleton remained more convex than in males. The palatal plane descended significantly away from the Frankfort horizontal plane. The anterior and posterior nasal spines moved caudally at about the same rate. They found that the SNA angle remained relatively stable with age, while the SNB angle increased significantly from adolescence into early adulthood. Because of this growth difference, they also found that most young adults experienced age-related decreases in the ANB angle.

Akgul and Toygar (2002) performed a longitudinal study of the craniofacial changes in 30 untreated subjects (16 males, 14 females) in the third decade of life. The mean age at the beginning of the observation period was approximately 22 years, and the total observation period was approximately 10 years. They found that the most significant changes occurred in the vertical dimension and that, predictably, the soft tissue measurements reflected the vertical changes in the underlying skeleton. Anterior facial height showed significant increases in both sexes, with lower facial height showing the greatest increase. The downward displacements of Nasion and Sella in relation to the Frankfort horizontal plane were significant in both sexes. The posterior movement of Sella was significant only in the men $(\mathrm{P}<0.001)$. The cranial base angle (Na-Se-Ba) showed a decrease in both sexes, but the decrease was statistically significant only in women $(\mathrm{P}<0.05)$.

Driscoll-Gilliland, Buschang and Behrents (2001) recorded seven cephalometric measurements to quantify the skeletal changes in the untreated sample detailed in the previous section. These results are shown in detail in Table 2-5. Their results showed that changes in SNA over an approximate 9 year period were small and not statistically significant in either sex. Interestingly, SNB showed a statistically significant increase in males $(P<0.01)$, but there was no statistically significant change in females. As shown in other studies, upper 
Table 2-5. Comparison of skeletal changes for untreated subjects (T1-T2).

\begin{tabular}{|c|c|c|c|c|c|c|}
\hline \multirow[b]{2}{*}{ Variable } & \multicolumn{3}{|c|}{ Females } & \multicolumn{3}{|c|}{ Males } \\
\hline & Mean & SD & Sig & Mean & SD & Sig \\
\hline SNA & 0.0 & 1.0 & NS & 0.3 & 0.9 & NS \\
\hline SNB & -0.2 & 0.9 & NS & 1.4 & 1.2 & ** \\
\hline N-ANS & 0.8 & 1.1 & * & 1.9 & 1.0 & ** \\
\hline ANS-Me & 2.3 & 1.5 & ** & 4.3 & 2.4 & ** \\
\hline Ar-Go & 2.7 & 2.1 & ** & 7.0 & 3.6 & ** \\
\hline MPA & -0.1 & 1.3 & NS & -3.4 & 2.4 & ** \\
\hline $\begin{array}{l}\text { Mandibular } \\
\text { rotation }\end{array}$ & 0.3 & 2.0 & NS & -1.7 & 2.7 & * \\
\hline
\end{tabular}

Notes: All angular measurements are in degrees $\left(^{\circ}\right)$. All linear measurements are in millimeters (mm). SD, standard deviation. Sig, significance. NS, not statistically significant. ${ }^{*} \mathrm{P}<0.01$. ${ }^{* *} \mathrm{P}<0.001$.

Source: Driscoll-Gilliland J, Buschang MA, Behrents RG. An evaluation of growth and stability in untreated and treated subjects. Am J Orthod Dentofacial Orthop 2001;120:588-97. 
anterior facial height (Na-ANS), lower anterior facial height (ANS-Me), and posterior facial height (Ar-Go), increased significantly in both sexes. The mandibular plane angle decreased a small but statistically insignificant amount in females, while males experienced a highly significant decrease $(\mathrm{P}<0.001)$ of $3.4^{\circ}$. Forward mandibular (bite-closing) rotation was not statistically significant in females, while males showed a significant $(\mathrm{P}<0.01)$ amount of forward mandibular rotation of $1.7^{\circ}$.

Björk and Skieller (1972) performed a longitudinal implant study on the facial development and tooth eruption of 21 untreated subjects confined to a 6-year interval around puberty. The general features of facial development consisted of a marked forward rotation of the face, including the two jaws, with greater forward rotation occurring in the mandible. The eruptive paths of the consisted of a marked forward rotation of the face, including the two jaws, with greater forward rotation occurring in the mandible. The eruptive paths of the posterior teeth essentially followed the rotation of the two jaws. At the lower border of the mandible about one half of the rotation was masked by a compensatory remodeling in this area. At the posterior border of the ramus about four-fifths of the mandibular rotation was masked by compensatory remodeling. The rotation of the maxilla was likewise masked by remodeling of the nasal floor, which remained almost unchanged in inclination.

Among their findings were that maxillomandibular convergence increases with age as described by decreases in MP-SN angle. The gonial angle of the mandible was also found to decrease with age, and this change was thought to be associated with the forward rotation of the mandible.

\section{Soft Tissue Profile Changes in Untreated Normal Occlusions}

Pecora, Bacetti and McNamara (2008) suggest that the soft tissue changes that occur as the result of normal age-related growth are among the most consistent changes that are seen over a long period in adults. They found that the soft tissues showed consistent growth from late adolescence (mean age $=17$ years) into late adulthood (mean age $=57$ years). Their results showed significant thinning and elongation of the upper lip. In addition, structural changes in the nose occurred, including drooping of the nasal tip and columella, which resulted in a more acute nasolabial angle.

Bishara, Hession and Peterson (1985) described the changes in Holdaway's soft-tissue angle, Merrifield's Z angle, upper and lower lip relationships to Ricketts' esthetic plane, and two angles of facial convexity in 35 untreated normal occlusions between the ages of 5 and 25 years of age. The 
subjects consisted of 20 males and 15 females from the Facial Growth Study of the University of Iowa for whom lateral cephalograms were taken semi-annually from ages 5 to 12 years, annually through 17 years of age, and once at 25 years of age. For the purposes of the current study, we will consider the findings reported between the ages of 17 years and 25 years. They found that Holdaway's soft tissue angle showed consistent decreases with age in 34 out of the 35 subjects, with males showing the most significant decreases. Decreases in Holdaway's soft tissue angle during this time period are mostly attributed to the continued forward growth of the mandible and forward rotation of the mandible. Merrifield's $Z$ angle showed increases with age in 28 out of the 35 subjects, with the other 7 subjects demonstrating either no change or slight decreases in $\mathrm{Z}$ angle. Increases in this angle are attributable to the retrusion of the lips with age and to continued forward movement of the mandible. They found that the distances of the upper and lower lips to Ricketts' esthetic plane (Pr-Pg') increased significantly and consistently in both sexes from 17 to 25 years of age, with males showing greater increases in both distances. Increases in these distances can be attributed to retrusion of the upper and lower lip as well as to the forward growth of the nose. From the perspective of the orthodontist, this is considered a critical age-related growth pattern, since treating an adolescent patient to adult norms might result in a soft tissue profile that could be considered unfavorable in adulthood. They also found that the angle of facial convexity decreased in 27 of the 35 subjects, while it either demonstrated no change or increased in the other eight subjects. Total facial convexity (which includes the nose) showed consistent increases with aging in all subjects.

Ochoa and Nanda (2004) noted that the facial profiles of males are more likely to become straighter as they progress into early adulthood due to an increase in prominence of the soft tissue Pogonion. They reported that females were more likely to maintain convex facial profiles due to less incremental growth of the mandible.

Behrents (1985) made several astute observations on adult changes in the soft tissue profile in his longitudinal studies on adult craniofacial growth. He suggested that while the nasolabial angle is a serious concern for an orthodontist when formulating a treatment plan for an adolescent, the nasolabial angle actually tends to improve if given enough time. He also suggested that individuals will appear to be less protrusive as they age due to four factors: the maxillary incisors are uprighting with age; the vertical dimension increases; the nose elongates; and the lips experience retrusion as well as a thinning of the upper lip. 


\section{Long Term Posttreatment Changes}

Having reviewed the changes expected as a result of normal growth and some of the factors known to influence the stability of craniofacial changes, we can now turn our attention to the long-term posttreatment craniofacial changes documented in previous studies.

\section{Skeletal Changes Following Treatment}

Harris, Gardner and Vaden (1999) described a longitudinal cephalometric study that examined posttreatment craniofacial changes over the long term. The study consisted of 36 subjects who received orthodontic treatment as adolescents and were recalled at an average of 5.5 years posttreatment for the first recall and 14.4 years for the second recall. Table 2-6 summarizes their findings.

The length of the midface (Cd-A) showed significant increases at the first and second recall examinations. These changes were attributed to the downward and forward growth of the maxilla. While Frankfort-mandibular angle (FMA) remained statistically unchanged during treatment, it decreased significantly by the first recall examination, with no statistical change in between the first and second recall examinations. Other significant posttreatment skeletal changes included large increases in mandibular length (Cd-Gn), decreases in the angle of convexity (Nasion-A-Pogonion angle) resulting in a flattening of the facial profile, increases in Articulare-Gnathion (Ar-Gn) distance, increases in lower anterior facial height (ANS-Me, PP-Me), and increases in posterior facial height (Ar-Go, Cd-Go, Se-Go). The increases in lower anterior facial height were attributed to normal facial growth since FMA decreased (which lessened the increase in facial height). The increases in posterior facial height were believed to reflect ramus growth and gonial remodeling. They concluded that most of the skeletal linear measurements showed significant changes within the first 5 years posttreatment, while maxillary and mandibular growth continued into early adulthood, and vertical growth continued well into the late recall period. The results suggest that the majority of orthodontic relapse of skeletal changes, if it occurs, is most likely to occur soon after treatment and that little relapse takes place beyond the first few years.

Ahn and Schneider (2000) performed a longitudinal study of posttreatment vertical changes in adult orthodontic patients. The study consisted of 33 patients who experienced at least 1 degree of clockwise rotation of the mandible during treatment who were recalled at an average of 5.6 years posttreatment. Mandibular clockwise rotation was quantified by angular changes in the $\mathrm{Y}$-axis measured cephalometrically. They found that the mandibular 
Table 2-6. Mean changes in cephalometric values.

\begin{tabular}{lccc}
\hline \multicolumn{1}{c}{ Variable } & $\begin{array}{c}\text { Mean Intreatment } \\
\text { Change }\end{array}$ & $\begin{array}{c}\text { Mean Change } \\
\text { Post-TX } \\
\text { to Recall 1 }\end{array}$ & $\begin{array}{c}\text { Mean Change } \\
\text { Post-TX } \\
\text { to Recall 2 }\end{array}$ \\
\hline ANB angle & -2.15 & -0.17 & 0.61 \\
ANS-Menton & 3.30 & 1.66 & 0.28 \\
Articulare-Gnathion & 6.20 & 3.94 & 0.65 \\
Articulare-Gonion & 4.13 & 2.87 & 0.60 \\
Condylion-A point & -0.36 & 3.00 & 1.13 \\
Condylion-Gnathion & 6.16 & 3.93 & 0.99 \\
Condylion-Gonion & 4.31 & 3.93 & 0.99 \\
Frankfort-mandibular angle & -0.06 & -1.63 & 0.21 \\
Nasion-A-Pogonion angle & -6.26 & -0.99 & 1.09 \\
Palatal plane-Menton & 4.80 & 1.74 & 0.39 \\
Sella-Gonion & 4.95 & 3.48 & 1.07 \\
\hline
\end{tabular}

Notes: Angular measurements are in degrees, and linear measurements are in millimeters.

Source: Harris EF, Gardner RZ, Vaden JL. A longitudinal cephalometric study of postorthodontic craniofacial changes. Am J Orthod Dentofacial Orthop 1999;115:77-82. 
clockwise rotation produced by orthodontic treatment relapsed by $24.7 \%$ after treatment. They concluded that while there was significant bite opening that can be expected to be lost following treatment over the long term, the proportion of the vertical effects that can be expected to remain is much larger.

\section{Dental Changes Following Treatment}

Udhe, Sadowdsky and BeGole (1983) studied posttreatment adult changes in the dentition of 72 patients with pretreatment Class I or Class II malocclusions. The sample consisted on 18 Class I extraction patients, 18 Class I nonextraction patients, 9 Class II extraction patients, and 27 Class II nonextraction patients who were recalled at a minimum of 12 years after treatment. Anteroposterior molar relationship changes were small, with a mean change of less than $0.5 \mathrm{~mm}$ for most groups. Overbite showed significant increases in all groups, with slightly greater increases in Class II patients. Overjet increases were significant in all groups and increased twice as much in Class II patients as it did in Class I patients. There was a tendency for the maxillary arch to develop minor crowding after treatment, while there was significantly more crowding in the mandibular arch. Table 2-7 summarizes their findings.

Little, Reidel and Årtun (1988) compared the pretreatment, end of treatment, 10 year posttreatment, and 20 year posttreatment records of 31 four premolar extraction cases to evaluate stability of mandibular anterior alignment. They found that crowding increased significantly during the first ten years following treatment and continued to increase, although to a lesser degree, in the 10 to 20 year period following treatment. At the end of the 20 year evaluation period, only $10 \%$ of the cases were judged to have clinically acceptable mandibular alignment.

Bärbel, Fishbach and Schwarze (1996) performed a longitudinal study on posttreatment changes in arch width dimensions. Their sample included 226 extraction and nonextraction cases with various malocclusions and a mean pretreatment age of 11.3 years. Long-term recall records were taken at a minimum of 10 years postretention. Their findings indicate that all arch width dimensions constrict significantly after treatment regardless of the treatment modality. Maxillary intermolar width showed the greatest amount of relapse of any dimension measured, with a total relapse of $26 \%$. Mandibular intercanine widths relapsed an average of $24 \%$, followed by $19 \%$ in mandibular intermolar width, and $14 \%$ in maxillary intercanine width.

Little, Reidel and Stein (1990) performed a longitudinal study on posttreatment changes in mandibular arch length following treatment by 
Table 2-7. Mean posttreatment changes in dental measurements.

\begin{tabular}{lcccc}
\hline \multicolumn{1}{c}{ Variable } & $\begin{array}{c}\text { Class I } \\
\text { Non-Extraction }\end{array}$ & $\begin{array}{c}\text { Class I } \\
\text { Extraction }\end{array}$ & $\begin{array}{c}\text { Class II } \\
\text { Non-Extraction }\end{array}$ & $\begin{array}{c}\text { Class II } \\
\text { Extraction }\end{array}$ \\
\hline $\begin{array}{l}\text { A-P molar relationship } \\
\quad \text { Left }\end{array}$ & 0.44 & 0.22 & 0.24 & 0.68 \\
$\quad$ Right & 0.19 & 0.07 & 0.67 & 1.05 \\
Overjet & 0.33 & 0.67 & 1.11 & 0.57 \\
Overbite & 0.94 & 1.16 & 1.60 & 1.02 \\
Crowding & & & & \\
$\quad$ Maxilla & -0.42 & -0.25 & -0.24 & -0.50 \\
$\quad$ Mandible & -1.17 & -1.23 & -1.07 & -1.33 \\
\hline
\end{tabular}

Notes: Measurements are in millimeters. Negative values indicate crowding.

Source: Udhe MD, Sadowsky C, BeGole EA. Long term stability of dental relationships after orthodontic treatment. Angle Orthod 1983;53:240-52. 
procedures known to increase arch length, which included the use of active lingual arches, lip bumpers, and removable appliances. The sample included 26 patients treated in the mixed dentition by graduate residents at the University of Washington who had diagnostic records taken at pretreatment, at the end of treatment, and at a minimum of 6 years posttreatment. At the end of active treatment, all 26 cases demonstrated a minimum of $1 \mathrm{~mm}$ in arch length gain and were considered satisfactory with minimal incisor irregularity index scores $($ mean $=2.10 \mathrm{~mm})$. At recall examination, the arch length was shorter than that at the end of active treatment in all patients. Out of 26 patients, 20 demonstrated a net loss of $1 \mathrm{~mm}$ or more of the total arch length gained by treatment. In 23 of the 26 patients, incisor irregularity index score was considered clinically unsatisfactory (mean $=6.0 \mathrm{~mm}$ ) at long term posttreatment, indicating poor stability of mandibular incisor positions. Mandibular intercanine width was also significantly reduced in 23 out of the 26 patients.

Elms, Buschang and Alexander (1996) performed a longitudinal study on the posttreatment changes experienced by patients with Class II division 1 malocclusions treated with nonextraction cervical-pull facebow headgear therapy. Cervical-pull facebow treatment was chosen for the patients in this sample to increase maxillary arch length through the distalization of maxillary molars and to restrict further maxillary growth. The sample included 42 white patients between the ages of 7 to 14 years of age (median age $=11.5$ years) at the start of treatment. Diagnostic records were taken at pretreatment, at the end of treatment, and at an average of 6.5 years posttreatment. At the end of active treatment, all 42 patients were considered satisfactory with minimal incisor irregularity index scores (mean $=1.90 \mathrm{~mm}$ ), while maxillary arch lengths were held very close to the pretreatment arch lengths and did not change significantly due to treatment. At long term posttreatment, maxillary arch length was, on average, $1 \mathrm{~mm}$ less than that at the end of active treatment. Incisor irregularity index score, however, was still considered clinically satisfactory (mean $=2.0 \mathrm{~mm}$ ) at long term posttreatment, indicating excellent stability of maxillary incisor positions. Maxillary intercanine width showed no significant change at the recall examination.

Sadowsky et al. (1994) studied long-term changes in the dentition of 22 nonextraction patients with significantly prolonged retention. The sample consisted of 9 Class I patients and 13 Class II patients with a mean age of 10.9 years at the start of treatment. Retention consisted of a maxillary Hawley retainer worn only at night and a fixed lower lingual retainer (from canine to canine) for a mean retention period of 8.4 years. All 22 patients were held in retention for a minimum of 5 years following treatment. Long-term postretention records were taken at least 5 years out of retention (mean $=6.3$ years). All dental variables showed some degree of relapse at the long-term 
postretention examination. Maxillary and mandibular irregularity indices increased from $0.9 \mathrm{~mm}$ and $1.0 \mathrm{~mm}$, respectively, at the time of retention removal to $2.0 \mathrm{~mm}$ and $2.4 \mathrm{~mm}$, respectively, at long-term postretention. Overbite and overjet both relapsed by $16 \%$. Mandibular canines lost $50 \%$ of the expansion achieved during treatment, mandibular first premolars lost $45 \%$, mandibular second premolars lost $31 \%$, and mandibular molars lost $17 \%$ of the expansion achieved. Maxillary canines, maxillary first premolars, and maxillary second premolars lost an insignificant amount of the expansion achieved at long-term postretention, while maxillary molars lost $17 \%$. Sadowsky et al. concluded that, when comparing mandibular incisor irregularity in this sample to that reported in most of the articles reviewed on studies of samples without prolonged retention, prolonged retention has a strong positive association with posttreatment incisor stability. They also concluded that expansion of all maxillary arch widths due to treatment are likely to have excellent stability following the removal of prolonged retention, while mandibular intercanine width expansion does not remain stable, even following prolonged retention.

\section{Soft Tissue Profile Changes Following Treatment}

Zierhut et al. (2000) performed a study on the long-term profile changes associated with successfully treated extraction and nonextraction Class II division 1 malocclusions. The sample consisted of 63 patients treated as adolescents that were evaluated at pretreatment, posttreatment, and long-term posttreatment (mean $=14$ years posttreatment) with lateral cephalometric radiographs. Statistically significant differences occurred from posttreatment to long term recall, but these were not different between extraction and nonextraction groups. The upper and lower lips became more retrusive relative to the E plane and to the S plane, but these changes were due to nose growth and forward movement of the soft tissue chin. They found that the ideal long-term lip positions suggested by Ricketts (1957) were not consistent with those found in the sample. While Ricketts found that the upper lip was $4 \mathrm{~mm}$ and the lower lip was $2 \mathrm{~mm}$ behind the E plane in esthetically pleasing profiles, those in the sample were more retrusive and closer to the values reported for normal, untreated adults of similar ages. Progressive flattening of the facial profile was observed for both groups, but this was also attributed to maturational changes associated with mandibular growth and nose growth.

Fudalej (2008) detailed the long-term changes in upper lip position relative to the incisal edge. The study was comprised of 54 subjects with lateral cephalometric radiographs taken at the end of orthodontic treatment (T1), at 10 years posttreatment (T2), and at 20 years posttreatment (T3). He found that Stomion-Incision superius (St-Is) distance decreased by $1.3 \mathrm{~mm}$ in males and 
showed no significant change in females from T1 to T2. However, females eventually caught up with the males during the next decade. Males experienced an additional decrease of $1.0 \mathrm{~mm}$ from T2 to T3, while females showed a decrease of $1.5 \mathrm{~mm}$ during that same time period. It was concluded that elongation of the upper lip continues throughout life and eventually exceeds the vertical growth of the face.

Singh (1990) performed a longitudinal study on changes in soft-tissue chin thickness after orthodontic treatment. The sample consisted of 31 male and 29 female patients that had orthodontic treatment completed at an average age of 14.5 years. All 60 subjects were recalled at a minimum of 5 years posttreatment (mean age at recall $=21.5$ years) for lateral cephalometric radiographs. All patients were identified according to facial type on the basis of cephalometric analysis as brachyfacial, mesofacial, or dolichofacial. Posttreatment and longterm recall cephalograms revealed that, in all patients, soft-tissue chin thickness increased at all six points measured around the symphysis. Males exhibited a greater increase in chin thickness than females in all dimensions with females only showing significant changes in chin thickness within the dolichofacial subgroup. Dolichofacial types showed the greatest increases in chin thickness in both sexes, with dolichofacial males exhibiting an increase from $0.9 \mathrm{~mm}$ to 1.4 $\mathrm{mm}$ measured at posttreatment to $1.6 \mathrm{~mm}$ to $2.5 \mathrm{~mm}$ measured at long-term recall (78-79\% increase). Mesofacial types showed the least change in both sexes. 


\section{CHAPTER 3. MATERIALS AND METHODS}

\section{Sample Description}

The data consisted of the posttreatment and recall lateral cephalograms taken of 30 females who had been treated orthodontically without premolar extractions. The 30 subjects included in this sample came from a pool of approximately 400 patients (all of whom started treatment between 1988 and 1999). All patients in the original pool were contacted by mail with a letter requesting their participation in the study. A sum of $\$ 25.00$ was offered to any patient willing to participate. All subjects included in the study had presented with a Class II division 1 malocclusion at the pretreatment examination. All subjects were treated in adolescence in the practice of a single experienced orthodontist, Dr. Richard A. Williams of Southaven, Mississippi. The average age at the end of treatment was 15.9 years, and the average age at recall was 28.3 years. The ages of each subject at the posttreatment and recall examinations are illustrated graphically in Figure 3-1.

The average recall duration was approximately 12.5 years $($ minimum $=$ 7.51 years; maximum $=18.99$ years). All 30 subjects received comprehensive orthodontic treatment with fixed labial appliances in the upper and lower arches, and all subjects were treated only once (no retreatments). All subjects were been treated with straightwire appliances (MBT prescription brackets and bands with 0.022" slots and molar tubes). All subjects were considered phenotypically normal at the pretreatment examination, in that they had no history of congenital disease or any identifiable syndrome. All subjects received upper and lower Hawley retainers for full-time wear for the first 6 months following treatment. None of the subjects received any form of fixed retention. The sample was one of convenience, and inclusion was based on the exclusion criteria outlined above as well as the subject's compliance to undergo a recall examination.

\section{Cephalometric Analysis}

The analog cephalograms from the posttreatment examination were scanned using a UMAX Powerlook III flatbed scanner at $300 \mathrm{dpi}$ and 256 gray scale, and the scans were saved as TIFF files. The radiographs in TIFF format were then imported into the Dolphin Imaging Cephalometric Tracing and Analysis module (Version 10; Dolphin Imaging \& Management Solutions, Chatsworth, CA). All cephalograms from the recall examination were in digital format, and thus, easily imported into the Dolphin Imaging Cephalometric Tracing and Analysis module. A custom cephalometric analysis was created on 


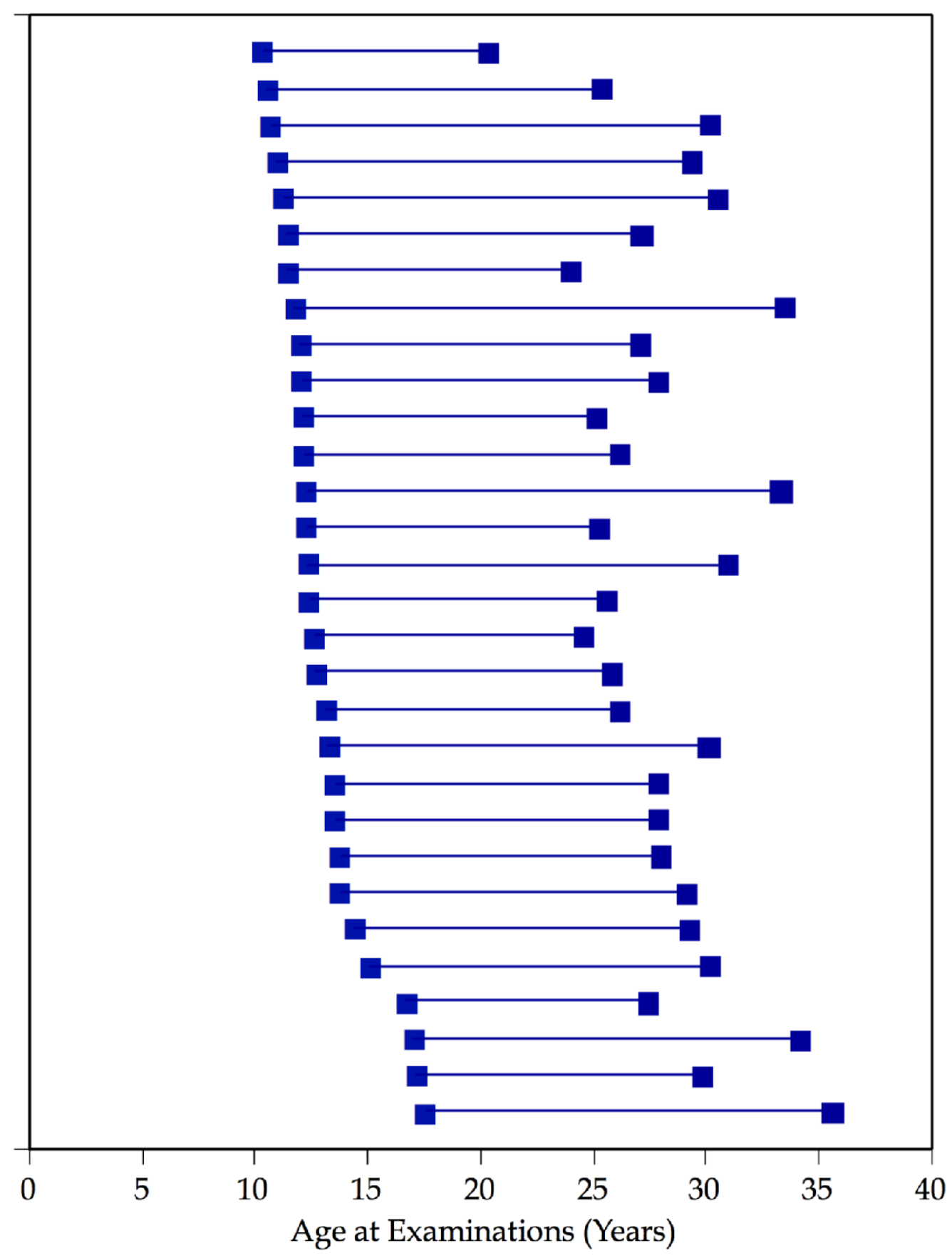

Figure 3-1. Ages of subjects at the posttreatment and recall examinations. 
the Dolphin Imaging software. The variables can broadly be grouped into three categories of skeletal, dental, and integumental dimensions.

The skeletal and soft-tissue landmarks used in this study are diagrammed in Figure 3-2. Dental landmarks are diagrammed in Figure 3-3. Most of the landmarks were traced digitally by landmark identification using Dolphin Imaging software. The following landmarks were traced by hand on acetate tracing paper: W point, Distal L6, and Distal U6. All measurements involving any of those three landmarks were performed manually with a millimetric ruler, whereas all measurements involving only landmarks that were traced digitally were performed by the Dolphin Imaging Cephalometric Tracing and Analysis module. For all bilateral anatomic landmarks in which there was a discrepancy between the left and right images, the midpoint between the locations of the right and left points was used. To assess the local maxima and minima, all films were oriented with Frankfort Horizontal parallel to a horizontal plane of reference before tracing.

There had been a change in cephalometers from the end of treatment to the recall examination. However, no information regarding the original cephalometer was available except that it was a Wehmer Rotating Anode. The current cephalometer is a Planmeca Promax (Planmeca Oy, Helsinki, Finland). The magnification of the Planmeca Promax, as reported in the technical manual, is $13 \%$. The American standard for orthodontics prior to digital radiography placed the patient's midsagittal plane at 60 inches $(152.4 \mathrm{~cm})$ from the source and the patient $15 \mathrm{~cm}$ from the film (Cohen 2005). According to Cohen, any cephalometer that uses these standardized dimensions magnifies lateral cephalometric radiographs by approximately $9.8 \%$. There was no way to know if these standardized dimensions were the same as those for the posttreatment cephalometer (or if these dimensions remained constant for all patients). This study assumes that this was the approximate magnification error of the original cephalometer.

To account for magnification error in cephalometric analysis, the "simple method" described by Cohen (2005) was used as a guide to calibrate all cephalograms to $0 \%$ magnification. A step-by-step description of the method used in this study is given below:

1. Scan the conventional (posttreatment) cephalometric radiograph with a ruler visible using a high-resolution (medical quality) flatbed scanner.

2. Digitize the conventional (posttreatment) image. Dolphin Imaging software will not allow for importation of a nondigitized cephalogram 


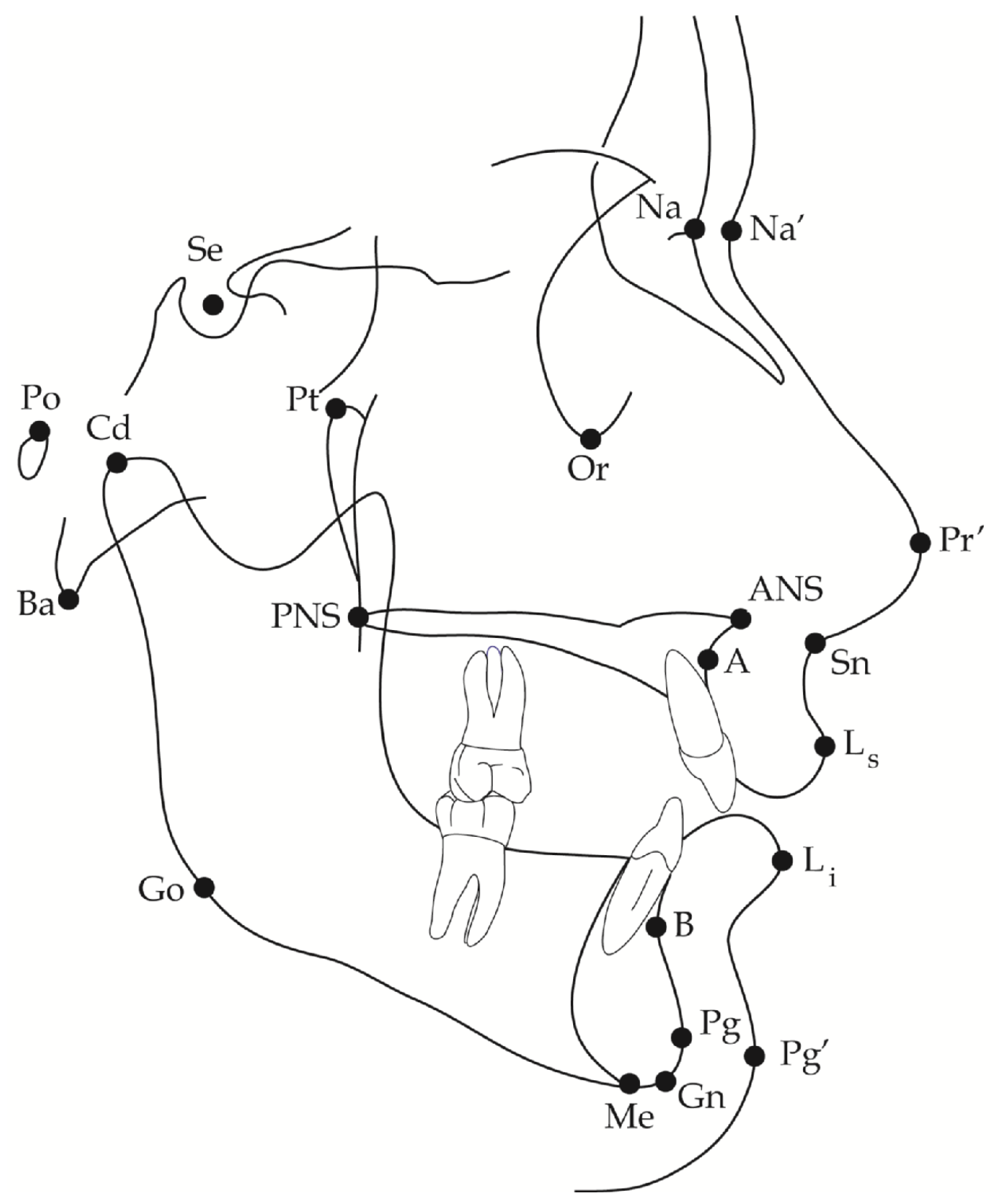

Figure 3-2. Cephalometric skeletal and soft-tissue landmarks. 


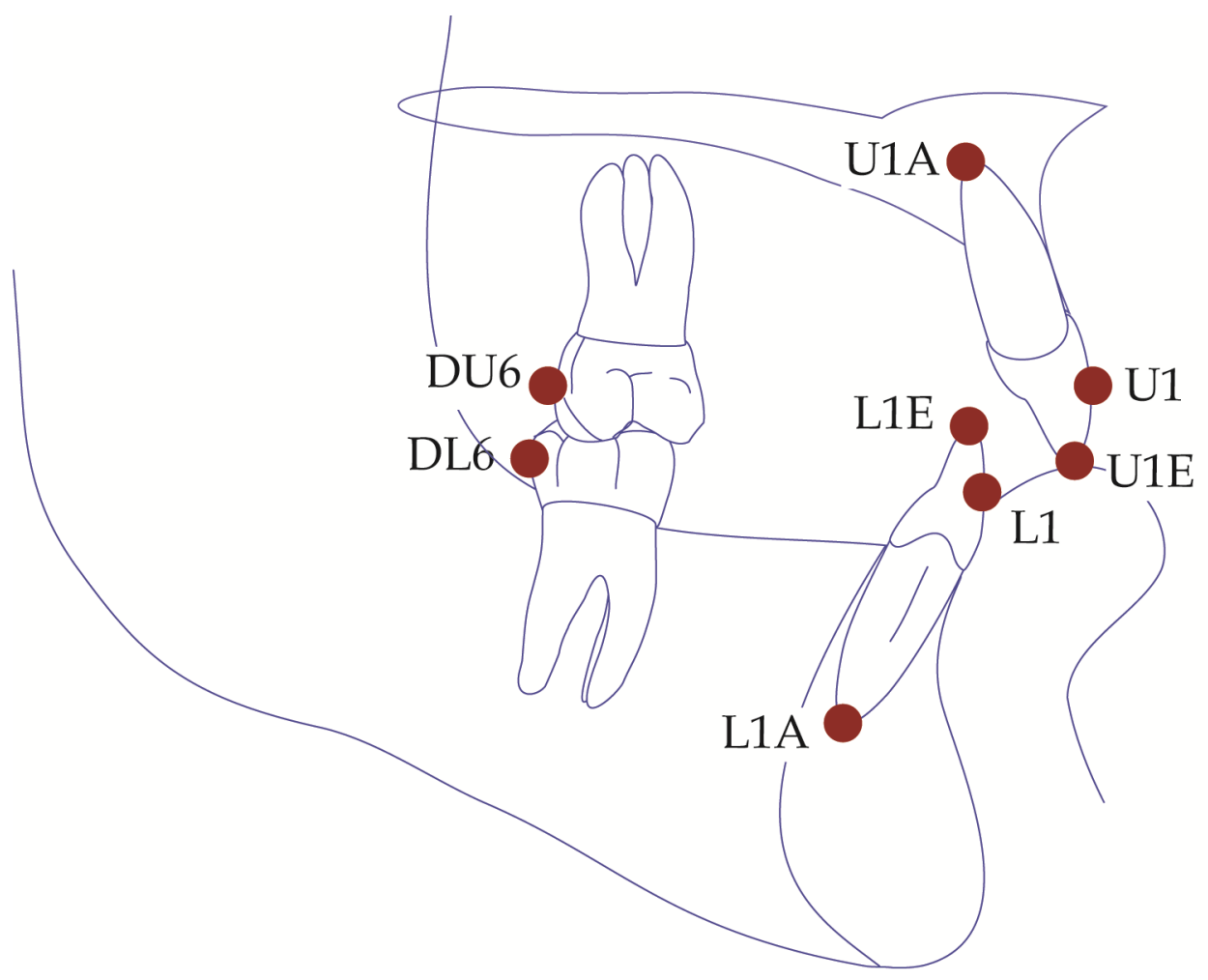

Figure 3-3. Cephalometric dental landmarks. 
because it has no idea how large the cephalogram is without registering its size during the digital analysis.

3. Import the newly digitized conventional (posttreatment) cephalometric image into Dolphin Imaging.

4. Import the digital image of the recall cephalometric radiograph into Dolphin Imaging. This image should already have a ruler visible from the head positioner of the cephalostat.

5. At this point, an important distinction needs to be made between the ruler on the conventional cephalogram and the ruler in the digital cephalogram. On the conventional posttreatment cephalogram, the ruler is added after the x-ray is taken. Since it is not magnified, and it cannot be used for direct calibration of the cephalogram. However, the digital recall cephalogram has a ruler included during the $\mathrm{x}$-ray. Therefore, the ruler is already magnified by the magnification of the cephalostat (13\%). Thus, calibrating the digital cephalogram by using the ruler from the head positioner will calibrate the cephalogram to $0 \%$ magnification.

6. An image magnified at $9.8 \%=1.098 \mathrm{X}$ the actual size of the image. Thus, calibration of the posttreatment cephalogram to $0 \%$ magnification requires reduction of the image size in Dolphin by $9.8 \%$. Given that 1.0/1.098 = 0.9107, the actual size of the image (1.0) equals the magnified image size (1.098) multiplied by this amount $(1.0=1.098 \times 0.9107)$. To accomplish this size reduction in Dolphin, the total length of the ruler scanned on top of the radiograph $(150 \mathrm{~mm})$ will need to be pre-set to a length of 136.61 $\mathrm{mm}(150 \mathrm{~mm} \times 0.9107=136.61 \mathrm{~mm})$. This is easily accomplished in Dolphin by setting the distance between "ruler point 1" and "ruler point 2 " to this length in the Digitize Setup dialogue box. When tracing the cephalogram, the "ruler point 1 " landmark is then placed at the $0 \mathrm{~mm}$ mark on the ruler, and "ruler point 2" is placed on the $150 \mathrm{~mm}$ mark on the ruler. Once all of the cephalometric landmarks have been identified and the OK button has been pressed, the posttreatment image is effectively calibrated to $0 \%$ magnification.

7. Calibrating the recall cephalogram to $0 \%$ magnification by using the ruler from the head positioner is simple, because the ruler is already magnified by the magnification of the cephalostat $(13 \%)$. This means that by presetting the distance between "ruler point 1" and "ruler point 2 " to its actual length $(45 \mathrm{~mm})$ before tracing, the radiograph will also be reduced to its actual size. 
The tracings for every cephalogram were repeated by the same examiner to test for intraexaminer repeatability error. Following comparison of the values measured for each cephalometric measurement for the initial and repeated tracings, a comparison of landmark identification was made between the two sets of tracings for all measurements that showed a deviation of greater than 0.5 degrees or $0.5 \mathrm{~mm}$. This comparison was done using the "overlay mode" in Dolphin Imaging, so there direct visualization of the locations of the landmarks for both tracings. In cases where there was of a deviation of greater than 0.5 between measurements for the first and second tracings, any landmark for the specific measurement in question that deviated from its correct location was retraced in "overlay mode" to reduce intraexaminer repeatability error. All measurements were entered in a Microsoft Excel (2003) spreadsheet to allow for the calculation of descriptive statistics.

The following alphabetical list provides definitions of the landmarks used in this study:

- A Point (Subspinale): The most posterior point on the curve of the maxilla between the anterior nasal spine and supradentale.

- ANS (Anterior Nasal Spine): The spinous process of the maxilla forming the most anterior projection of the floor of the nasal cavity.

- B Point (Supramentale): The most posterior point on the bony curvature of the mandible between Infradentale and Pogonion.

- $\mathrm{Cd}$ (Condylion): The most superior-posterior point on the curvature of the condyle.

- DL6 (Distal Lower 6): The point at the most distal aspect of the mandibular first molar.

- DU6 (Distal Upper 6): The point at the most distal aspect of the maxillary first molar.

- DOP (Downs Occlusal Plane): The line that bisects the maxillary and mandibular incisal overbite and the most anterior occlusal contact between the maxillary and mandibular first molars.

- E-Plane: The line formed between Pronasale and soft tissue Pogonion

- Go (Gonion): The most posterior-inferior point on the gonial angle of the mandible. 
- Gn (Gnathion): The anatomical midpoint between Pogonion and Menton on the anterior border of the mandible

- Ii (Incision Inferius): The incisal tip of the most anterior mandibular central incisor.

- Is (Incision Superius): The incisal tip of the most anterior maxillary central incisor.

- L1 (Lower Incisor): The most ventral point on the labial surface of the mandibular central incisor.

- L6 (Lower Molar): The point located at the mesiobuccal cusp tip of the mandibular first molar.

- L1A (Lower Incisor Apex): The point located at the root apex of the mandibular central incisor.

- L1E (Lower Incisor Edge): The point located at the incisal edge of the mandibular central incisor.

- Lower Lip: The most ventral point located on the lower lip

- $\operatorname{Li}$ (Labrale Inferius): The point where the boundary of the vermilion border of the lower lip and the skin is intersected by the median sagittal plane.

- Ls (Labrale Superius): The point on the upper lip lying in the median sagittal plane on a line drawn across the boundary of the vermilion border and skin.

- Me (Menton): The most inferior point on the mandibular symphysis.

- $\mathrm{Na}$ (Nasion): The junction of the frontal nasal suture at the most posterior point on the curvature at the bridge of the nose.

- Na Perp (Nasion Perpendicular): The vertical line drawn perpendicular to the Frankfort horizontal plane that intersects Nasion.

- Or (Orbitale): The lowest point on the inferior margin of the bony orbit. In the instance where the right and left orbits are not located at the same 
level, the inferior margin of the orbit is considered to be located at a level that is equidistant to the inferior margins of the right and left orbit.

- (Posterior Nasal PNS Spine): The spinous process formed by the most posterior projection of the juncture of the palatine bones in the midline of the roof of the oral cavity.

- Po (Porion): The midpoint on the superior rim of the external auditory meatus.

- Pg (Pogonion): The most anterior point on the anterior contour of the bony chin.

- Pg' (Soft Tissue Pogonion): The most anterior point on the anterior contour of the soft tissue chin.

- $\operatorname{Pr}$ (Pronasale): The most anterior point on the soft tissue nose.

- Pt (Pterygomaxillary Fissure): The most superior-posterior point on the average of the right and left outlines of the pterygomaxillary fissures.

- PtV (Pterygoid Vertical): The vertical line drawn perpendicular to Frankfort horizontal plane through the most posterior-superior point on the best fit of the right and left outlines of the pterygomaxillary fissures.

- Se (Sella Turcica): The midpoint of the hypophyseal fossa, determined by inspection.

- Sm' (Soft Tissue Supramentale): The point of greatest concavity in the midline of the lower lip between labrale inferius and soft tissue pogonion.

- St (Stomion): The point of intersection of the upper and lower lips.

- Sn (Subnasale): The most posterior inferior point on the lower border of the nose, where it meets the upper border of the upper lip

- U1 (Upper Incisor): The most ventral point on the labial surface of the maxillary central incisor.

- U1A (Upper Incisor Apex): The point located at the root apex of the maxillary central incisor. 
- U1E (Upper Incisor Edge): The point located at the incisal edge of the maxillary central incisor.

- U6 (Upper Molar): The point located at the mesiobuccal cusp tip of the maxillary first molar.

- Upper Lip: The most ventral point located on the upper lip

- W Point: The point located on the most posterior (dorsal) aspect of the curvature of the mandibular symphysis.

The following linear distances and angles were calculated for each lateral cephalogram. This list below (in alphabetical order) provides definitions of the measurements that were used in this study. Graphical representations of measurements are illustrated in Appendix D.

- ANB: The inferior angle formed at the junction of the Nasion-A Point line and the Nasion-B Point line (Figure D-25).

- AO-BO (Wits Appraisal): The linear distance between two points along Downs' occlusal plane obtained from the intersection of a perpendicular line from point $A$ and from point $B$ to the occlusal plane (Figure D-26).

- Cd-A: The linear distance from Condylion to A Point (Figure D-7).

- Cd-Gn: The linear distance from Condylion to Gnathion (Figure D-20).

- Cd-Go: The linear distance from Condylion to Gonion (Figure D-21).

- Distal U6-PTV: The linear distance from the most distal aspect of the upper first molar to Pterygoid Vertical (Figure D-46).

- Distal U6-U1 (Maxillary Arch Length): The linear distance between the most distal aspect of the maxillary first molar and the labial surface of the maxillary central incisor (Figure D-48).

- Distal L6-L1 (Mandibular Arch Length): The linear distance between the most distal aspect of the mandibular first molar and the labial surface of the mandibular central incisor (Figure D-47).

- Distal L6-W point: The linear distance between the most distal aspect of the lower first molar and $\mathrm{W}$ point (Figure D-45). 
- DOP-FH: The angle between the Frankfort Horizontal plane and Downs occlusal plane. When the occlusal plane is tipped down in the front, the angle is positive. When the occlusal plane is tipped up in the front, so the two lines intersect in the front portion of the face, the angle is negative (Figure D-22).

- E Plane-Li: The linear distance from Labrale superius to E Plane (Figure D-51).

- E Plane-Ls: The linear distance from Labrale superius to E Plane (Figure D-50).

- FH-Na-Pg: The linear distance between Nasion and Pogonion when projected perpendicular to Frankfort Horizontal plane (Figure D-29).

- FMA: The anterior inferior-angle formed at the junction of the Frankfort Horizontal plane and the mandibular plane (Figure D-27).

- FMIA: The posterior-inferior angle formed between the Frankfort Horizontal plane and the long axis of the mandibular incisor (Figure D-38).

- Go-Pg: The linear distance from Gonion to Pogonion (Figure D-22).

- IMPA: The posterior-superior angle formed at the junction of the mandibular plane and the long axis of the mandibular incisor (Figure D-37).

- L1-NB ${ }^{\circ}$ : The inferior angle formed from a line through the long axis of the mandibular incisor (L1E-L1A) and the Nasion-B point line (Figure D-35).

- L1-NB mm: The linear distance from Incision Inferius (Ii) to the Nasion-B point line (Figure D-36).

- L1-A-Pg: The linear distance between L1E and the A Point-Pogonion Line (Figure D-43).

- L1-W Point: The linear distance between L1E and W point (Figure D-44).

- Ls-Sn-Pr: The nasolabial angle; The anterior angle formed at the point of intersection of the labrale superius-subnasale line and subnasalepronasale line. (Figure D-52). 
- Li-B'-Pg': The mentolabial angle; The anterior angle formed at the point of intersection of labrale inferius-soft tissue B point line and soft tissue B point-soft tissue pogonion line (Figure D-53).

- Me-PP: The linear distance from Menton to Palatal Plane measured along a line that intersects palatal plane at a $90^{\circ}$ angle (Figure D-9).

- Na-A-Pg: The superior angle formed by the junction of the Nasion-A Point and the A Point-Pogonion line (Figure D-28).

- Na-ANS: The linear distance from Nasion to Anterior Nasal Spine (Figure D-5).

- Na-Ba: The linear distance between Nasion and Basion (Figure D-3).

- Na-Me: The linear distance between Nasion and Menton (Figure D-10).

- FH-Na-Pg: The posterior-inferior angle formed between the NasionPogonion plane and the Frankfort Horizontal line (Figure D-29).

- Na-PP: The linear distance from Nasion to Palatal Plane measured along a line that intersects palatal plane at a $90^{\circ}$ angle (Figure D-8).

- Na-Se-Ba: The anterior angle formed by the Sella to Nasion line and the Sella to Basion line (Figure D-4).

- NaPerp-A: The linear distance from point A to Nasion when projected perpendicular to the Frankfort Horizontal plane (Figure D-14).

- NaPerp-B: The linear distance from point B to Nasion when projected perpendicular to the Frankfort Horizontal plane (Figure D-18).

- NaPerp-Pg: The linear distance from Pogonion to Nasion when projected perpendicular to the Frankfort Horizontal plane (Figure D-24).

- NaPerp-Pr: The linear distance from Pronasale to Nasion when projected perpendicular to the Frankfort Horizontal plane (Figure D-56).

- Overbite: The vertical distance from U1E to L1E (Figure D-39).

- Overjet: The horizontal distance from U1E to L1E (Figure D-40). 
- PFH/AFH: The ratio of the posterior facial height to the anterior facial height.

- PP-SN: The angle formed between the palatal plane and Sella-Nasion line (Figure D-17).

- PTV-A: The linear distance from Pterygoid Vertical and A point (Figure D-15).

- PTV-B: The linear distance from Pterygoid Vertical and B point (Figure D-19).

- Se-A: The linear distance from Sella to A point (Figure D-16).

- Se-Go: The linear distance from Sella to Gonion (Figure D-11).

- Se-Ba: The linear distance from Sella to Basion (Figure D-2).

- Se-PNS: The linear distance between Sella and Posterior Nasal Spine (Figure D-6).

- Se-Na: The linear distance from Sella to Nasion (Figure D-1).

- Sn-Li: The linear distance from Subnasale to Labrale Inferius (Figure D-55).

- Sn-Ls: The linear distance from Subnasale to Labrale Superius (Figure D-54).

- SNA: The posterior inferior angle formed at the junction of the SellaNasion line and the Nasion-A Point line (Figure D-12).

- SNB: The posterior inferior angle formed at the junction of the SellaNasion plane and the Nasion-B Point line (Figure D-13).

- U1-A-Pg: The linear distance from U1E to the A Point-Pogonion Line (Figure D-42).

- U1-L1 ${ }^{\circ}$ : The posterior angle formed by a line through the long axis of U1 and a line through the long axis of L1 (Figure D-31). 
- U1-NA ${ }^{\circ}$ : The superior angle formed from a line through the long axis of $\mathrm{U} 1$ and the Nasion-A point line (Figure D-33).

- U1-NA mm: The linear distance from Is to the Nasion-A point line (Figure D-34).

- U1-PtV: The linear distance between the labial surface of the maxillary central incisor and Pterygoid Vertical (Figure D-41).

- U1-SN: The posterior-inferior angle formed by a line through the long axis of U1 to the line Sella-Nasion (Figure D-32).

- Y-Axis: The anterior-inferior angle formed by the intersection of the Frankfort Horizontal line to the line from Sella to Gnathion (Figure D-23).

- Z-Angle: The posterior-inferior angle formed by Frankfort Horizontal and the line from soft tissue pogonion to the most protrusive lip (Figure D-49).

- W Point-Pg': The linear distance from W Point to soft tissue Pogonion (Figure D-57).

The cephalometric measurements described above are categorized (Table 3-1) into skeletal, dental, and soft tissue measurements along with the purpose for each measurement in the cephalometric analysis.

\section{Statistical Analysis}

Data were collated into an Excel ${ }^{\circledR}$ spreadsheet (Microsoft Corporation, Redmond, WA) then transferred to the JMP ${ }^{\circledR}$ statistical package version 9.0 (SAS Institute Inc., Cary, NC). Exploratory data analysis (Tukey 1977) was performed, searching for outliers; those due to technical errors were corrected. Descriptive statistics (Sokal and Rohlf 1995) were computed, including the arithmetic mean $(\bar{x})$, standard deviation (sd), standard error of mean (sem), upper and lower $95 \%$ confidence limit $\left(\mathrm{L}_{1}, \mathrm{~L}_{2}\right)$, sample size $(\mathrm{n})$, sample variance $\left(\mathrm{s}^{2}\right)$, skewness $\left(\mathrm{g}_{1}\right)$, kurtosis $\left(\mathrm{g}_{2}\right)$, coefficient of variation ( $\left.\mathrm{cv}\right)$, number of cases missing, maximum value, median value (50th percentile), and minimum value.

Change in size of each cephalometric dimension was calculated as the recall value minus the posttreatment value, so positive differences reflect increases and negative differences denote decreases in size between the examinations. 
Table 3-1. Cephalometric measurement abbreviations and corresponding measurement descriptions.

\begin{tabular}{|c|c|}
\hline Abbreviation & Description \\
\hline $\begin{array}{l}\text { Se-Na } \\
\text { Se-Ba } \\
\text { Na-Ba } \\
\text { Na-Se-Ba }\end{array}$ & \begin{tabular}{l}
\multicolumn{1}{c}{ Cranial Base } \\
Anterior cranial base length $(\mathrm{mm})$ \\
Posterior cranial base length $(\mathrm{mm})$ \\
Total cranial base length $(\mathrm{mm})$ \\
Cranial base angle $\left(^{\circ}\right)$
\end{tabular} \\
\hline $\begin{array}{l}\text { Na-ANS } \\
\text { Se-PNS } \\
\text { Cd-A }\end{array}$ & $\begin{array}{l}\text { Midface } \\
\text { Vertical height of the anterior midface }(\mathrm{mm}) \\
\text { Vertical height of the posterior midface }(\mathrm{mm}) \\
\text { Horizontal length of the midface }(\mathrm{mm})\end{array}$ \\
\hline $\begin{array}{l}\text { Na-PP } \\
\text { Me-PP } \\
\text { Na-Me } \\
\text { Se-Go } \\
\text { PFH/AFH }\end{array}$ & $\begin{array}{l}\quad \text { Facial Height } \\
\text { Upper anterior facial height }(\mathrm{mm}) \\
\text { Lower anterior facial height }(\mathrm{mm}) \\
\text { Total anterior facial height }(\mathrm{mm}) \\
\text { Posterior facial height }(\mathrm{mm}) \\
\text { Ratio of posterior facial height to anterior facial } \\
\text { height }\end{array}$ \\
\hline SNA & $\begin{array}{l}\text { Maxilla } \\
\text { Positional change in the maxilla relative to } \\
\text { anterior cranial base }\left(^{\circ}\right)\end{array}$ \\
\hline Na Perp-A & A-P positional change in the maxilla (mm) \\
\hline PtV-A & A-P positional change in the maxilla (mm) \\
\hline Se-A & $\begin{array}{l}\text { Downward \& forward directional growth of } \\
\text { the maxilla }(\mathrm{mm})\end{array}$ \\
\hline PP-SN & Rotation of the palatal line $\left(^{\circ}\right)$ \\
\hline SNB & $\begin{array}{l}\text { Mandible } \\
\text { Positional change in the mandible relative to } \\
\text { the anterior cranial base }\left(^{\circ}\right)\end{array}$ \\
\hline Na Perp-B & A-P positional change in the mandible (mm) \\
\hline $\mathrm{PtV}-\mathrm{B}$ & A-P positional change in the mandible (mm) \\
\hline Cd-Gn & Mandibular length (mm) \\
\hline Cd-Go & Vertical mandibular ramus length (mm) \\
\hline Go-Pg & Mandibular corpus length (mm) \\
\hline Y-Axis & Rotation of the mandible $\left(^{\circ}\right)$ \\
\hline Na Perp-Pg & Protrusive growth of the chin $(\mathrm{mm})$ \\
\hline
\end{tabular}


Table 3-1. Continued.

\begin{tabular}{|c|c|}
\hline Abbreviation & Description \\
\hline & Maxillomandibular Relationships \\
\hline ANB & A-P relationship of the maxilla-mandible $\left(^{\circ}\right)$ \\
\hline $\mathrm{AO}-\mathrm{BO}$ & A-P relationship of the maxilla-mandible (mm) \\
\hline FMA & Maxillomandibular divergence $\left(^{\circ}\right)$ \\
\hline Na-A-Pg & Facial convexity $\left(^{\circ}\right)$ \\
\hline FH-Na-Pg & Facial angle $\left({ }^{\circ}\right)$ \\
\hline DOcc Plane-FH & Rotation of occlusal plane $\left(^{\circ}\right)$ \\
\hline $\mathrm{U} 1 / \mathrm{L} 1$ & $\begin{array}{l}\text { Dental Changes } \\
\text { Angular relationship between the maxillary \& } \\
\text { mandibular central incisors }\left(^{\circ}\right)\end{array}$ \\
\hline $\mathrm{U} 1 / \mathrm{SN}$ & $\begin{array}{l}\text { Angulation of the maxillary central incisor relative } \\
\text { to the cranial base }\left(^{\circ}\right)\end{array}$ \\
\hline $\mathrm{U} 1-\mathrm{NA}^{\circ}$ & $\begin{array}{l}\text { Angulation of the maxillary central incisor relative } \\
\text { to the maxilla }\left(^{\circ}\right)\end{array}$ \\
\hline U1-NA mm & $\begin{array}{l}\text { Position of the maxillary central incisor relative to } \\
\text { the maxilla }(\mathrm{mm})\end{array}$ \\
\hline L1-NB ${ }^{\circ}$ & $\begin{array}{l}\text { Angulation of the mandibular central incisor } \\
\text { relative to the mandible }\left(^{\circ}\right)\end{array}$ \\
\hline L1-NB mm & $\begin{array}{l}\text { Position of the mandibular central incisor relative to } \\
\text { the mandible }(\mathrm{mm})\end{array}$ \\
\hline IMPA & $\begin{array}{l}\text { Inclination of lower incisors relative to the } \\
\text { mandibular plane }\left({ }^{\circ}\right)\end{array}$ \\
\hline FMIA & $\begin{array}{l}\text { Inclination of lower incisors relative to Frankfort } \\
\text { horizontal }\left({ }^{\circ}\right)\end{array}$ \\
\hline Overbite & Vertical overlap of the upper \& lower incisors $(\mathrm{mm})$ \\
\hline Overjet & Horizontal overlap of upper \& lower incisors (mm) \\
\hline U1-A-Pg & A-P movement of the maxillary central incisor ( $\mathrm{mm}$ ) \\
\hline $\mathrm{U} 1-\mathrm{PtV}$ & A-P movement of the maxillary central incisor $(\mathrm{mm})$ \\
\hline L1-A-Pg & A-P movement of mandibular central incisor $(\mathrm{mm})$ \\
\hline L1-W point & A-P movement of mandibular central incisor ( $\mathrm{mm}$ ) \\
\hline L6-W point & A-P movement of the mandibular first molar ( $\mathrm{mm})$ \\
\hline U6-PtV & A-P movement of the maxillary first molar (mm) \\
\hline Distal L6-L1 & Mandibular arch length (mm) \\
\hline Distal U6-U1 & Maxillary arch length (mm) \\
\hline
\end{tabular}


Table 3-1. Continued.

\begin{tabular}{ll}
\hline Abbreviation & \multicolumn{1}{c}{ Doft Tissue Profile } \\
\hline Z Angle & Protrusiveness of lips $\left(^{\circ}\right)$ \\
E Plane-Ls & Protrusiveness of the upper lip (mm) \\
E Plane-Li & Protrusiveness of the lower lip (mm) \\
Ls-Sn-Pr & Nasolabial angle $\left(^{\circ}\right)$ \\
Li- $-B^{\prime}-P^{\prime}$ & Mentolabial angle $\left(^{\circ}\right)$ \\
Sn-Ls & Length of the upper lip (mm) \\
Sn-Li & Drooping of the lower lip (mm) \\
Na Perp-Pr & A-P growth of the nose (mm) \\
W point-Pg' & A-P growth of the soft tissue chin $(\mathrm{mm})$ \\
\hline
\end{tabular}


The principal question was whether a cephalometric dimension changed significantly from the end of treatment to the long-term recall examination. All of the subjects were female, so "sex" did not need to be controlled in the tests. All of the measurements were made twice, but the method of remeasurement was not random, so repeatability errors - the technical error of measurement(though tested) were trivially small. The most informative tests were simply the one-sample t-tests of whether the change (from end of treatment to the recall examination) differed significantly from zero (e.g., Sokal and Rohlf 1995). For completeness, these two-tail t-tests were calculated separately for the first and the second sets of measurements, but the results were invariably the same. The usual level of significance (alpha $=0.05$ ) was used, and no correction was made for multiple comparisons.

Repeatability was quantified using the conventional Dahlberg statistic (Dahlberg 1940), which is:

$$
\sqrt{\frac{\sum\left(\mathrm{X}_{1 \mathrm{i}}-\mathrm{X}_{2 \mathrm{i}}\right)^{2}}{2 \mathrm{n}}}
$$

where $\mathrm{X}_{1 \mathrm{i}}$ and $\mathrm{X}_{2 \mathrm{i}}$ are the pairs of repeated measurements and $\mathrm{n}$ is the number of measurements (Houston 1983). 


\section{CHAPTER 4. RESULTS}

The focus of this study was to quantify the skeletodental changes of former patients viewed cephalometrically from posttreatment to the recall examination. As outlined in the sample description, all 30 patients in the sample were females, and they all exhibited a Class II division 1 malocclusion at the pretreatment examination. The average patient at posttreatment can be characterized as having a mildly retrognathic profile $\left(\mathrm{ANB}=3.5^{\circ} ; \mathrm{Na}-\mathrm{A}-\mathrm{Pg}=\right.$ $\left.3.5^{\circ}\right)$. The cases were treated in their adolescence, and the average recall duration was approximately 12.5 years $($ minimum $=7.5$ years, maximum $=19.0$ years).

\section{Descriptive and Inferential Statistics}

Tables of the descriptive and inferential statistics are presented in Appendices A and B. Appendix A contains the descriptive statistics and tests for significant posttreatment changes based on repeated measurements. Appendix B contains the results of the mixed-model analysis of variance (ANOVA) testing for posttreatment changes. Graphical representations of the distributions of posttreatment change among individuals for each cephalometric variable are presented in Appendix C.

Cephalometric studies commonly report the mean changes, but, even when the standard deviation also is reported, the reader gains little sense of the nature of the variability among subjects. In order to gain a better perspective on the distributions of change, the posttreatment changes of each of the variables are graphed in Appendix C, generally using one-millimeter or one-degree intervals (though some changes made this scale impractical). These histograms provide a visual sense of how dispersed the individuals are in the sample. When the changes are all clumped close together, the mean is representative of how the "average" patient responded. When, however, the changes are dispersed across several millimeters or degrees, there is more individual (and less systematic) response following treatment. Since the sample is reasonably homogenous (e.g., all are girls with Class II nonextraction cases treated by one specialist), the breadth of the responses among cases is of interest, because they show the interindividual responses to effectively the same treatment. These graphs also disclose outliers, which raises the question of why those particular people changed so much.

To enhance the readability of this document, the description of the posttreatment changes were divided into the following sections so that they can 
be compared with similar measures within the craniofacial complex: cranial base, midface, facial height, maxilla, mandible, maxillomandibular relationships, dental changes, and soft tissue profile changes.

\section{Cranial Base}

The distance from Sella to Nasion increased by an average of $2.7 \mathrm{~mm}(\mathrm{P}<$ 0.0001). All of the subjects in the sample were women, and they all exhibited positive growth, with most (70\%) of the cases experiencing between 2 and $3 \mathrm{~mm}$ of growth (Figure C-1). This lengthening of the anterior cranial base may be related to the involution of the frontal sinuses with age. Of course, Nasion is on the frontal bone, so it strictly is not part of the endochondral cranial base. Prior studies show that the increases in Sella-Nasion distance in late adolescence and adulthood are due to bony apposition of the ectocranial surface of the frontal bone, which moves Nasion to the ventral relative to Sella (Knott 1971). The growth found here is comparable to the findings of Sinclair and Little (1985) who found an average increase of $1.6 \mathrm{~mm}$ in the Sella-Nasion length of 32 untreated females with 'normal occlusions' over a seven year interval from 12-13 to 19-20 years of age. When comparing that sample to the 30 females in the current sample, the mean change for the current sample was observed at an older age and over an interval that was longer in duration by an average of 5.5 years (mean recall $=12.5$ years).

There was an average increase in Sella-Basion length of approximately 2.2 $\mathrm{mm}$, which is a statistically significant amount of growth of the posterior cranial base $(\mathrm{P}<0.0001)$. In analyzing the distribution of changes among individuals, five of the subjects in this sample showed no change, while rather dramatic increases (> $4 \mathrm{~mm}$ ) were observed in four other subjects (Figure C-2). The amount of change observed for the latter four subjects probably was due to growth at the spheno-occipital synchondroses (rather than from remodeling of the clivus). Information on when the spheno-occipital synchondroses fuses is sparse and variable (Melsen 1969; Knott 1971) but fusion (and cessation of elongation of the posterior cranial base) seems to occur in girls in the early teens, which ought to coincide with the conclusion of orthodontic treatment. Our speculation is that the age of fusion of the spheno-occipital synchondroses is variable, with fusion occurring later in some individuals than in others. Another potential cause of the change in the Sella-Basion distance, as mentioned, is bony apposition along the dorsal aspect of the clivus.

Total cranial base length as measured from Nasion to Basion increased by an average of $5.5 \mathrm{~mm}(\mathrm{P}<0.0001)$. In contrast, changes in the Cranial Base Angle (Na-Se-Ba) were found to be extremely small and statistically insignificant 
$(\mathrm{P}>0.85)$. As a whole, these changes indicate lengthening of the cranial base both to the ventral and to the dorsal, which discloses an overall increase in head size. The findings of Israel on the aging craniofacial skeleton (1968) give validation to these changes. Israel found that the cranial base, along with most other cranial components, showed increases in size on the order of about five to seven percent from adolescence into late adulthood. Consistent with the current study, he found that the cranial base angle formed by the Sella to Nasion and Sella to Basion lines did not alter with age. This lack of systematic change in Cranial Base Angle (also often referred to as "saddle angle") is a finding shared with other studies (e.g., Lewis and Roche 1977).

\section{Midface}

The vertical height of the anterior midface (Na-ANS), the vertical height of the posterior midface (Se-PNS), and the horizontal length of the midface (Cd-A) exhibited average increases of $3.1 \mathrm{~mm}, 2.7 \mathrm{~mm}$, and $3.5 \mathrm{~mm}$, respectively. The mean changes in the linear dimensions of the midface were statistically significant $(\mathrm{P}<0.0001)$, suggesting positive growth in both the vertical and horizontal planes.

\section{Facial Height}

Upper anterior facial height (Na-PP) and lower anterior facial height (Me$\mathrm{PP})$ increased an average of $3.1 \mathrm{~mm}$ and $4.3 \mathrm{~mm}$, respectively $(\mathrm{P}<0.0001)$, combining to produce slightly more than $7 \mathrm{~mm}$ of growth in total anterior facial height following treatment. Posterior facial height (Se-Go) increased by $4.1 \mathrm{~mm}$ on average $(P<0.0001)$, which was slightly more than half of the total growth of the anterior face. Most of the growth during this posttreatment interval is likely to have occurred in the mid-to-late teens, and as other studies have shown. It is likely to have slowed substantially in the third decade of life (Harris, Gardner and Vaden 1999).

There was no statistically significant change in the ratio of posterior facial height to anterior facial height ( $\mathrm{PFH} / \mathrm{AFH}$ ) from posttreatment to long-term recall. The mean ratio at both posttreatment and recall was 0.67 , suggesting no significant change in proportionality between anterior and posterior facial height over the long-term. It is not surprising that this ratio remained so stable, since other studies have also found that facial height ratios remain stable in spite of the ongoing growth of the anterior and posterior face independently of one another (Nasjleti and Kowalski 1975). 


\section{Maxilla}

Positional change of the maxilla in relation to the anterior cranial base as measured by the Sella-Nasion-A point angle (SNA) exhibited an average increase of 0.4 degrees $(\mathrm{P}<0.0001)$. This was a small change, but since all subjects showed some positive increase, the change achieved statistical significance (Figure C-11). This change was very close in magnitude to the change found by Sinclair and Little (1985) in their untreated sample in which the SNA angle increased an average of 0.35 degrees over a seven year interval. This may suggest that maxillary growth occurs at a similar rate, whether the dentition is treated or untreated.

Horizontal positional change in the maxilla as measured by Nasion Perpendicular to A point (NaPerp-A) and by Pterygoid Vertical to A point (PtVA) was statistically significant for both linear measurements $(P<0.0001)$. NaPerp-A increased by approximately $1.4 \mathrm{~mm}$, while PtV-A increased by approximately $2.7 \mathrm{~mm}$. The differences between the changes measured for these two dimensions show how measurements using Nasion-Perpendicular as a reference plane tend to underestimate the forward growth of the jaws, because Nasion itself is remodeling forward, thus decreasing the measured distance between itself and A point. Knowledge of this fact would mean that the changes measured from Pterygoid Vertical (PtV-A) are likely to give a more accurate measure of the amount of maxillary growth that actually occurred. Both of these dimensions in conjunction with the increase in the SNA angle confirm a small, but statistically significant, forward growth of the maxilla.

Downward and forward growth of the maxilla as measured by the distance between Sella and A point (Se-A) was significant and increased by 4.3 mm on average $(\mathrm{P}<0.0001)$. The amount of change between subjects ranged from $3.6 \mathrm{~mm}$ to $5.0 \mathrm{~mm}$, with $60 \%$ of the sample falling within 4.0 to $4.4 \mathrm{~mm}$ (Figure C-14).

The angulation of the palatal plane relative to Sella-Nasion remained stable (mean change $=0.45^{\circ} ; \mathrm{P}=0.18$ ) on average, although the distribution of changes among individuals (Figure $\mathrm{C}-15$ ) revealed appreciable inter-individual variability. The stability of this angle is consistent with the observations of the amount of relative growth in the vertical height of the anterior midface and of the posterior midface. Knowing that these two dimensions increased by nearly the same length (within $0.4 \mathrm{~mm}$ ), one would not expect much change in the angulation of the palatal plane. 


\section{Mandible}

Positional change of the mandible in relation to the anterior cranial base as measured by the Sella-Nasion-B point angle (SNB) exhibited an average increase of 0.8 degrees $(\mathrm{P}<0.0001)$, and this change was systematic throughout the sample (Figure C-16). The observed change in SNB angle was approximately twice as great as in the SNA angle, disclosing greater forward growth of the mandible relative to the cranial base when compared to the maxilla.

Horizontal positional change in the mandible as measured by Pterygoid Vertical to B point (PtV-B) showed a significant average increase of $2.4 \mathrm{~mm}(\mathrm{P}<$ 0.0001). This, in conjunction with the increase measured in SNB angle, show that there was a statistically significant amount of forward growth of the mandible following treatment in adolescence.

Mandibular length (Cd-Gn), vertical mandibular ramus length (Cd-Go), and mandibular corpus length (Go-Pg) increased by $6.6 \mathrm{~mm}, 5.3 \mathrm{~mm}$, and 4.5 $\mathrm{mm}$ on average, respectively $(\mathrm{P}<0.0001)$. Collectively, these findings indicated significant growth of the mandible vertically, horizontally, and diagonally. Analysis of the distribution of change among individuals for these three measurements revealed a large range in amounts between individuals (Figures C-19, C-20, and C-21). Increases in mandibular length, for example, ranged from $1 \mathrm{~mm}$ to $11 \mathrm{~mm}$, but with nearly $50 \%$ of the sample falling within the $6-8 \mathrm{~mm}$ range. Vertical mandibular ramus length and mandibular corpus length showed similar ranges of change among individuals. The increase in mandibular corpus length was likely to be, in large part, a result of growth at the gonial process. The large range of observed changes in these three measurements suggests that prediction of posttreatment change in the mandible can be difficult.

The Y-Axis decreased by an average of 0.5 degrees. This decrease had marginal statistical significance $(P=0.05)$, and this suggested a trivial amount of counter-clockwise (bite-closing) rotation of the mandible following treatment. Overall, this result shows that the $\mathrm{Y}$-axis remained relatively stable over the long-term. The stability of the $\mathrm{Y}$-axis is in agreement with most other studies (Ricketts 1981). It cannot be known from these data whether this minor decrease is normative or whether it reflects settling of the dentition into greater cusp-tofossa interdigitations following treatment.

Prominence of the chin, measured as Nasion Perpendicular to Pogonion (NaPerp-Pg), increased by $2.0 \mathrm{~mm}$ on average $(\mathrm{P}<0.0001)$. Counter-clockwise rotation of the mandible (although minimally significant in this sample) could account for some of the positive change. Enlow and Harris (1964) show that 
Pogonion is appositional over time, which would account for some of the forward displacement of Pogonion.

\section{Maxillomandibular Relationships}

Positional change of the maxilla in relation to the mandible as measured by the A point-Nasion-B point angle (ANB) exhibited an average decrease of 0.35 degrees $(P<0.0001)$. The average decrease of $0.5 \mathrm{~mm}$ for the Wits Appraisal measurement (AO-BO) was in agreement with the decrease in ANB angle, but the change in $\mathrm{AO}-\mathrm{BO}$ was not significant statistically significant $(P=0.30)$. Analysis of the distribution of changes among individuals revealed that the changes in ANB angle were systematic throughout the sample, as all 30 females experienced a negative change in the ANB angle in the range of 0 to -1 degrees (Figure C-24). In contrast, Wits Appraisal (AO-BO) values did not show systematic change, as there was appreciable inter-individual variability (Figure C-25).

The Frankfort Horizontal to Mandibular Plane Angle (FMA), as mentioned, exhibited a small and insignificant $(P=0.20)$ decrease of 0.5 degrees. The changes in this angle were not systematic, but instead, showed a fair amount of inter-individual variability (Figure C-26). Much of the change in FMA can be attributed remodeling at Gonion, which is subject to varying intensities of muscle strain.

The angle measured between Downs' occlusal plane and the Frankfort Horizontal line revealed significant counter-clockwise rotation of the occlusal plane. The angle decreased by an average of 1.85 degrees $(P=0.0027)$. An explanation for such a change could be greater downward growth of the mandibular ramus in relation to the downward growth of the anterior mandible. Vertical growth of the mandibular ramus did exceed the increase in lower anterior facial height in this sample, which would lend some support to this. The settling of the dentition following treatment could also contribute to this change.

Even though Nasion, A point, and Pogonion all experienced forward growth, skeletal facial convexity measured as Nasion-A point-Pogonion (Na-A$\mathrm{Pg}$ ) did not change significantly $(\mathrm{P}=0.20)$, and thus remained stable on the average. The facial angle (FH-Na-Pg), in contrast, did show a statistically significant increase averaging 1.3 degrees $(P<0.0001)$. This showed that although facial convexity did not undergo any appreciable change, the mandible was displaced to a more forward position in relation to the forehead. The "trend" cited in previous studies is that the facial angle is likely to become more 
orthognathic with age (Forsberg 1979), which is a finding supported by the present results.

\section{Dental Changes}

The angular relationship between the upper and lower incisors, the interincisal angle (U1-L1), experienced significant change. This angle increased from an average of 119 degrees at posttreatment to 125 degrees at long-term recall for a mean increase of about 6 degrees $(\mathrm{P}<0.0001)$. This was a favorable change that is related to the uprighting of the upper and lower incisors from a more proclined angulation at the end of treatment.

The angulation of the upper incisors decreased significantly as indicated by two measurements. The U1-SN angle decreased by an average of 3.1 degrees $(\mathrm{P}=0.003)$, while the U1-NA angle decreased by 3.5 degrees on the average $(\mathrm{P}=$ $0.0006)$. The linear measurement from the tip of the maxillary central incisor to the Nasion-A point line (U1-NA mm) did not change significantly $(\mathrm{P}=0.43)$, indicating no significant labial movement of the maxillary central incisor (at least none in excess of the forward remodeling of Nasion and A point).

The angulation of the lower incisors decreased significantly as indicated by three measurements. Lower Incisor-Mandibular Plane Angle (IMPA) decreased by an average of 4.1 degrees $(P=0.0013)$, Frankfort HorizontalMandibular Incisor Angle (FMIA) increased by 4.6 degrees $(\mathrm{P}=0.0003)$, and L1NB angle decreased by 2.7 degrees $(P=0.009)$. There was a fair amount of interindividual variability for these three measurements (Figures C-33, C-35, and C-36), but the vast majority of individuals experienced uprighting of the lower incisors rather than proclination. The linear measurement from the tip of the mandibular central incisor to the Nasion-B point line (L1-NB $\mathrm{mm}$ ) also exhibited a decrease averaging $1.3 \mathrm{~mm}(\mathrm{P}<0.0001)$.

Horizontal movement of the maxillary central incisor as measured from a reference line located to the ventral (U1-A-Pg) showed no statistically significant change $(P=0.95)$. Horizontal movement of the maxillary central incisor as measured from a posteriorly positioned reference line on the maxilla (U1-PtV) showed a highly significant increase of $2.9 \mathrm{~mm}$ of labial movement $(\mathrm{P}<0.0001)$ on average, but this dimension includes all horizontal maxillary growth. When accounting for maxillary growth, this suggests that the maxillary central incisor did not experience any significant movement within the supporting bone (maxilla). Instead, it moved forward relative to Pterygoid Vertical as the result of forward movement of the maxilla. 
Horizontal movement of the mandibular central incisor was measured from two reference points located mesial to the tooth. The measurement L1-A$\mathrm{Pg}$ showed that the lower incisor experienced an average labial movement of 0.9 $\mathrm{mm}(\mathrm{P}=0.0006)$. In contrast, the measurement L1-W point, which ought to be a more realistic measurement since the reference point is on the same bone as L1, did not show a statistically significant change $(P=0.70)$. This suggests that even though the A-Pg line is in common use, it may distort interpretation, since A point and Pogonion are themselves remodeling.

Overbite and overjet both increased significantly from posttreatment to the recall examination $(\mathrm{P}<0.0001)$. Overbite increased by an average of $0.9 \mathrm{~mm}$, and overjet increased by an average of $1.0 \mathrm{~mm}$. The increase in overbite can be explained, in part, by the observed uprighting of the maxillary and mandibular incisors. Analysis of the distributions of change among individuals revealed that all but four subjects $(26 / 30)$ showed a positive change in overbite, with greater than $75 \%$ of the subjects experiencing 0 -to- $2 \mathrm{~mm}$ of overbite deepening (Figure C-40). Similarly, all but five subjects (25/30) showed a positive change in overjet, with $63 \%$ of the subjects experiencing 0 -to- $2 \mathrm{~mm}$ of overjet increase (Figure C-41).

The horizontal position of the maxillary and mandibular first molars (parallel to Frankfort-Horizontal) showed statistically significant mesial movements $(\mathrm{P}<0.0001)$. The mandibular first molar exhibited an average mesial movement of $1.4 \mathrm{~mm}$ relative to an anteriorly positioned reference point on the mandible (L6-W point). This change was fairly systematic in that 27 out of 30 individuals experienced a decrease in this distance of zero to $-2 \mathrm{~mm}$ (Figure C-43). The maxillary first molar exhibited an average mesial movement of 2.4 $\mathrm{mm}$ relative to a reference point at the posterior limit of the maxilla (U6-PtV). The greater forward movement of the maxillary first molar in comparison to the lower first molar may occur because $\mathrm{PtV}$ is not on the same bone as $\mathrm{U} 6$ (whereas $\mathrm{W}$ point is on the same bone as L6). Unlike the mesial movement of the lower first molar, this change showed a high degree of inter-individual variability (Figure C-45). Mesial movement of maxillary and mandibular molars is considered a normative change that often occurs with aging (Sinclair and Little 1983; Harris 1997).

Maxillary and mandibular arch lengths each showed statistically significant decreases $(\mathrm{P}<0.0001)$ over time. Maxillary arch length (DU6-L1) decreased an average of $1.7 \mathrm{~mm}$, while mandibular arch length (DL6-L1) decreased an average of $1.2 \mathrm{~mm}$. Much of the decrease in arch length can be explained by the observed uprighting of the upper and lower incisors and by mesial movement of the posterior dentition. Arch length decreases, similar to mesial molar movement, have been found in both treated and untreated samples 
with normal occlusions, so this was also expected. Normal consolidation (plus any interstitial attrition) of the dentition with age is the most cited explanation for decreases in arch length.

\section{Soft Tissue Profile Changes}

Merrifield's Z Angle, a measure of lip position, measures overall lip prominence in relation to the anterior cranial base. In this sample, the $\mathrm{Z}$ Angle averaged $76.4^{\circ}$ at the end of treatment, and the average increased to $80.9^{\circ}$ by the recall examination. The overall change during this interval was an average increase of $4.5^{\circ}(\mathrm{P}<0.0001)$. The most likely explanation for the observed increase in this angle is that the chin becomes more protrusive in relation to the nose with age. This change is consistent with the increase in facial angle (FH-Na$\mathrm{Pg}$ ) that was also observed for this sample.

Protrusiveness of the upper lip measured from Ricketts' E-Plane (E planeLs) exhibited an average distance of $-4.4 \mathrm{~mm}$ at posttreatment, which is close to Ricketts' norm of $-4.0 \mathrm{~mm}$ (Ricketts 1957). The average distance at the recall examination had increased to a mean of $-5.1 \mathrm{~mm}$, indicating greater retrusion of the upper lip, with a mean decrease during this interval of $-0.7 \mathrm{~mm}(\mathrm{P}=0.0010)$.

Protrusiveness of the lower lip measured from Ricketts' E-Plane (E PlaneLi) exhibited an average distance of $-1.7 \mathrm{~mm}$ at posttreatment, which is close to Ricketts' norm of $-2.0 \mathrm{~mm}$. This distance receded farther than that of the upper lip during the examination, with the average distance at the recall examination increasing to a mean of $-2.9 \mathrm{~mm}$. The result was a mean change of $-1.2 \mathrm{~mm}(\mathrm{P}<$ $0.0001)$. The reduced protrusion of the upper and lower lips is an anticipated, normative change that has been reported in numerous studies (Singh 1990; Zierhut et al. 2000). Behrents (1985) attributed decreased lip protrusion, in part, to the uprighting of the upper and lower incisors with age, and this finding is supported by the current study.

The angular relationship of the upper lip to the nose measured as the Nasolabial Angle (Ls-Sn-Pr) did not show a significant change in this sample. One might expect this angle to increase over time, since it is known that the upper lip thins and becomes more flaccid with age (Finch and Schneider 1985), but the results of this study do not show this. It is also possible that the onset of gradual increases in the Nasolabial Angle do not occur until a period of adulthood later than that which the subjects in this study were followed. Mentolabial Angle ( $\mathrm{Li}^{-\mathrm{B}^{\prime}-\mathrm{Pg}}$ ), a measure of the angular relationship between the lower lip and the soft-tissue chin, also did not change. In combination, these results show that although the upper and lower lips become more retruded over 
the long-term, this did not significantly affect their angular relationships with the nose or soft-tissue chin, respectively.

Length of the upper lip (Sn-Ls) increased from a mean of $18.6 \mathrm{~mm}$ at posttreatment to a mean of $20.3 \mathrm{~mm}$ at recall, resulting in a net increase of 1.7 $\mathrm{mm}(\mathrm{P}<0.0001)$. Similarly, the lower lip also showed significant movement in a downward direction as measured by the millimetric distance between Subnasale and Labrale inferius (Sn-Li). This distance increased from an average of $25.5 \mathrm{~mm}$ at posttreatment to $27.8 \mathrm{~mm}$ at recall, resulting in a mean increase of $2.3 \mathrm{~mm}(\mathrm{P}<$ 0.0001). The observed drooping of the upper and lower lips was an expected change of the facial soft tissues with age, as they are known to become more flaccid as muscular tone decreases (Meema et al. 1973).

Forward growth of the nose (NaPerp-Pr) and of the soft tissue chin (W point-Pg') also showed significant age-related changes. The nose grew forward an average of $1.9 \mathrm{~mm}$, and the soft-tissue chin grew forward by $1.5 \mathrm{~mm}$ on average $(\mathrm{P}<0.0001)$. Both of these changes were anticipated as previous studies have shown similar findings (Singh 1990; Zierhut et al. 2000).

\section{Predictability of Posttreatment Change}

It is clinically valuable to know whether the amount of change following treatment is predictable. We investigated three different approaches to addressing this question. These approaches were (1) to regress the posttreatment change on the subject's age at the end of treatment, (2) to regress the posttreatment change on the duration (years) between the end of treatment and the recall examination, and (3) to use analysis of covariance to regress the posttreatment change on the subject's age at the end of treatment while controlling for the duration of treatment. We present the results from all three approaches, though the ANCOVA models are most reliable since they take into account more of the variability.

\section{End of Treatment}

One simple approach is to ask whether the subject's age at the end of treatment is predictive of the amount of posttreatment change. Age at the end of treatment is a measure of how far a subject has progressed toward (or into) biological maturity and, thus, how much subsequent growth can be anticipated. This was tested with linear regression analysis (e.g., Sokal and Rohlf 1995), with the subject's age at the end of treatment as the independent $(X)$ variable, and the amount of change between the end of treatment and the recall examination as the 
dependent $(\mathrm{Y})$ variable. Tests for each of the 59 variables are listed in Table 4-1. Statistical results for the $\mathrm{Y}$-intercept also are provided for completeness, but the relevant issue is whether the P-value for the regression coefficient achieved significance (alpha $=0.05)$.

Just 4 of the 59 tests is significant at $P<0.05$, namely NaPerp-A, NaPerp-B, Distal L6-W, and the Nasolabial Angle. One conclusion - right off - is that (as assessed from this battery of dimensions) most changes do not depend on the adolescent subject's age at treatment since there are so few significant associations.

The regression coefficient for NaPerp-A is positive $(0.29 \mathrm{~mm} /$ year $)$ as shown in Figure 4-1. Formally, the regression equation is

$$
\text { Change }=-3.10+0.29(\text { Age })
$$

where "change" is the posttreatment change in the distances from NasionPerpendicular to A point. The $\mathrm{P}$ value associated with the regression coefficient is 0.0089 .

This finding is surprising because it shows that -in this sample--the amount of forward growth in point $\mathrm{A}$ after treatment is greater in older adolescent patients, where subsequent growth ought to be less rather than more. Of note, these positive regression coefficients for age at the end of treatment both for Nasion-Perpendicular to A point and to B point persist (as discussed below) in the ANCOVA model where duration of treatment also is included in the model.

The second significant association is with NaPerp-B. Here, again, the association is caused by older teenagers exhibiting more growth following treatment (Figure 4-2). Of note, several cases prior to about 16 years of age exhibit no growth (or slightly negative change) in B point. The regression coefficient is 0.52 , which means that, on average, every year added to the age at the end of treatment contributes another $0.52 \mathrm{~mm}$ to the observed forward growth of $B$ point at the recall examination. Of note, both of these significant, positive associations (NaPerp-A and NaPerp-B) persist in the ANCOVA models (discussed below). Also, these measures of forward growth of points A and B are in excess of the forward growth of Nasion itself.

The third significant association identified in Table 4-1 is between age at the end of treatment and the distance between L6 and $\mathrm{W}$ point (Figure 4-3). The changes, except for the oldest subjects, are positive, meaning that this distance typically increases following treatment, but the amount of increase diminishes as 
Table 4-1. Results of linear regression analysis testing whether patient's age at end of treatment is predictive of the amount of post-treatment change.

\begin{tabular}{|c|c|c|c|c|c|c|}
\hline \multirow[b]{2}{*}{ Variable } & \multicolumn{3}{|c|}{ Y-Intercept } & \multicolumn{3}{|c|}{ Regression Coefficient } \\
\hline & Estimate & T-Test & P-Value & Estimate & T-Test & P-Value \\
\hline Se-Na & 3.30 & 4.63 & $\leq 0.0001$ & -0.04 & -0.82 & 0.4193 \\
\hline Se-Ba & -0.19 & -0.06 & 0.9545 & 0.15 & 0.73 & 0.4692 \\
\hline $\mathrm{Na}-\mathrm{Ba}$ & 1.73 & 0.66 & 0.5136 & 0.24 & 1.46 & 0.1546 \\
\hline $\mathrm{Na}-\mathrm{Se}-\mathrm{Ba}$ & 4.04 & 1.29 & 0.2078 & -0.25 & -1.29 & 0.2070 \\
\hline Na-ANS & 3.60 & 1.79 & 0.0839 & -0.03 & -0.24 & 0.8138 \\
\hline Se-PNS & 2.60 & 0.78 & 0.4430 & 0.01 & 0.04 & 0.9681 \\
\hline Cd-A & -0.49 & -0.17 & 0.8685 & 0.25 & 1.39 & 0.1751 \\
\hline $\mathrm{Na}-\mathrm{PP}$ & 3.60 & 1.79 & 0.0839 & -0.03 & -0.24 & 0.8138 \\
\hline Me-PP & 1.07 & 0.35 & 0.7320 & 0.21 & 1.06 & 0.2985 \\
\hline $\mathrm{Na}-\mathrm{Me}$ & 2.32 & 0.73 & 0.4691 & 0.31 & 1.55 & 0.1321 \\
\hline Se-Go & 6.03 & 1.06 & 0.2961 & -0.12 & -0.33 & 0.7402 \\
\hline PFH/AFH & 0.03 & 0.69 & 0.4964 & 0.00 & -0.81 & 0.4230 \\
\hline SNA & 0.79 & 2.94 & $\underline{0.0064}$ & -0.02 & -1.30 & 0.2029 \\
\hline NaPerp-A & -3.10 & -1.92 & 0.0654 & 0.29 & 2.81 & $\underline{0.0089}$ \\
\hline $\mathrm{PtV}-\mathrm{A}$ & 4.14 & 1.63 & 0.1148 & -0.09 & -0.57 & 0.5735 \\
\hline Se-A & 2.91 & 1.63 & 0.1134 & 0.09 & 0.81 & 0.4230 \\
\hline PP-SN & -1.02 & -0.35 & 0.7273 & 0.09 & 0.51 & 0.6132 \\
\hline SNB & 1.34 & 3.31 & 0.0026 & -0.04 & -1.42 & 0.1677 \\
\hline NaPerp-B & -5.50 & -1.91 & 0.0658 & 0.52 & 2.87 & 0.0077 \\
\hline $\mathrm{PtV}-\mathrm{B}$ & 1.87 & 2.45 & $\underline{0.0206}$ & 0.04 & 0.76 & 0.4555 \\
\hline Cd-Gn & 3.35 & 1.13 & 0.2665 & 0.20 & 1.10 & 0.2809 \\
\hline Cd-Go & 3.64 & 0.77 & 0.4453 & 0.11 & 0.36 & 0.7219 \\
\hline Go-Pg & 3.60 & 0.80 & 0.4286 & 0.06 & 0.20 & 0.8402 \\
\hline $\mathrm{Y}$-Axis & 1.84 & 0.91 & 0.3724 & -0.15 & -1.16 & 0.2555 \\
\hline NaPerp-Pg & -2.83 & -0.77 & 0.4457 & 0.31 & 1.33 & 0.1944 \\
\hline ANB & -0.54 & -1.20 & 0.2388 & 0.01 & 0.53 & 0.5972 \\
\hline $\mathrm{AO}-\mathrm{BO}$ & 1.60 & 0.41 & 0.6879 & -0.13 & -0.54 & 0.5946 \\
\hline FMA & 0.08 & 0.02 & 0.9834 & -0.04 & -0.17 & 0.8693 \\
\hline Na-A-Pg & 0.11 & 0.04 & 0.9715 & 0.02 & 0.11 & 0.9101 \\
\hline FH-Na-Pg & -2.01 & -0.91 & 0.3696 & 0.21 & 1.52 & 0.1397 \\
\hline U1/L1 & -2.95 & -0.30 & 0.7639 & 0.60 & 0.98 & 0.3337 \\
\hline U1/SN & 11.72 & 1.55 & 0.1316 & -0.94 & -1.98 & 0.0578 \\
\hline U1-NA & 11.06 & 1.47 & 0.1528 & -0.92 & -1.95 & 0.0606 \\
\hline U1-NA mm & 2.29 & 0.89 & 0.3791 & -0.16 & -0.99 & 0.3299 \\
\hline $\mathrm{L}^{1-\mathrm{NB}^{\circ}}$ & -8.84 & -1.02 & 0.3145 & 0.38 & 0.70 & 0.4925 \\
\hline L1-NB mm & -0.70 & -0.28 & 0.7796 & -0.04 & -0.26 & 0.7970 \\
\hline
\end{tabular}


Table 4-1. Continued.

\begin{tabular}{lrrlrrrr}
\hline & \multicolumn{3}{c}{ Y-Intercept } & & \multicolumn{3}{c}{ Regression Coefficient } \\
\cline { 2 - 4 } Variable & Estimate & T-Test & P-Value & & Estimate & T-Test & P-Value \\
\hline IMPA & -4.72 & -0.47 & 0.6420 & & 0.04 & 0.06 & 0.9551 \\
FMIA & 4.66 & 0.48 & 0.6315 & & 0.00 & -0.01 & 0.9958 \\
U1-APg & 0.33 & 0.18 & 0.8560 & -0.02 & -0.17 & 0.8636 \\
L1-APg & -0.65 & -0.32 & 0.7482 & -0.02 & -0.14 & 0.8869 \\
D Occ Pl-FH & -3.66 & -0.75 & 0.4594 & & 0.11 & 0.37 & 0.7152 \\
Overbite & 0.75 & 0.57 & 0.5753 & & 0.01 & 0.11 & 0.9110 \\
Overjet & 1.29 & 0.77 & 0.4476 & & -0.02 & -0.21 & 0.8387 \\
L1-W Pt & 0.50 & 0.30 & 0.7667 & -0.03 & -0.33 & 0.7407 \\
Distal L6-W Pt & -3.09 & -3.76 & 0.0008 & & 0.14 & 2.65 & $\mathbf{0 . 0 1 3 0}$ \\
U1-PtV & -0.45 & -0.16 & 0.8778 & & 0.21 & 1.19 & 0.2446 \\
U6-PtV & 1.84 & 0.66 & 0.5171 & & 0.03 & 0.19 & 0.8523 \\
Distal L6-L1 & 2.47 & 2.83 & 0.0086 & & -0.08 & -1.41 & 0.1694 \\
Distal U6-U1 & 2.07 & 1.97 & 0.0593 & & -0.02 & -0.33 & 0.7429 \\
Z Angle & 1.97 & 0.30 & 0.7649 & & 0.16 & 0.39 & 0.6997 \\
E Plane-Ls & -2.76 & -1.87 & 0.0722 & & 0.13 & 1.42 & 0.1658 \\
E Plane-Li & -1.80 & -0.97 & 0.3386 & & 0.04 & 0.33 & 0.7419 \\
Nasolabial & & & & & & & \\
Angle & 31.07 & 2.97 & 0.0060 & -1.94 & -2.96 & $\mathbf{0 . 0 0 6 2}$ \\
Li-A'-Pg' & -2.96 & -1.19 & 0.2458 & 0.08 & 0.50 & 0.6191 \\
Sn-StoS & 3.63 & 1.61 & 0.1179 & -0.12 & -0.87 & 0.3917 \\
Sn-Ls & 1.77 & 2.66 & 0.0129 & 0.00 & -0.04 & 0.9715 \\
Sn-Li & 6.02 & 1.76 & 0.0895 & -0.24 & -1.11 & 0.2764 \\
NaPerp-Pr & 2.29 & 2.28 & 0.0303 & -0.03 & -0.40 & 0.6889 \\
W Pt-Pg' & 3.17 & 3.53 & 0.0015 & -0.10 & -1.84 & 0.0769 \\
\hline
\end{tabular}

Note: Statistical significant regression coefficients are underlined. 


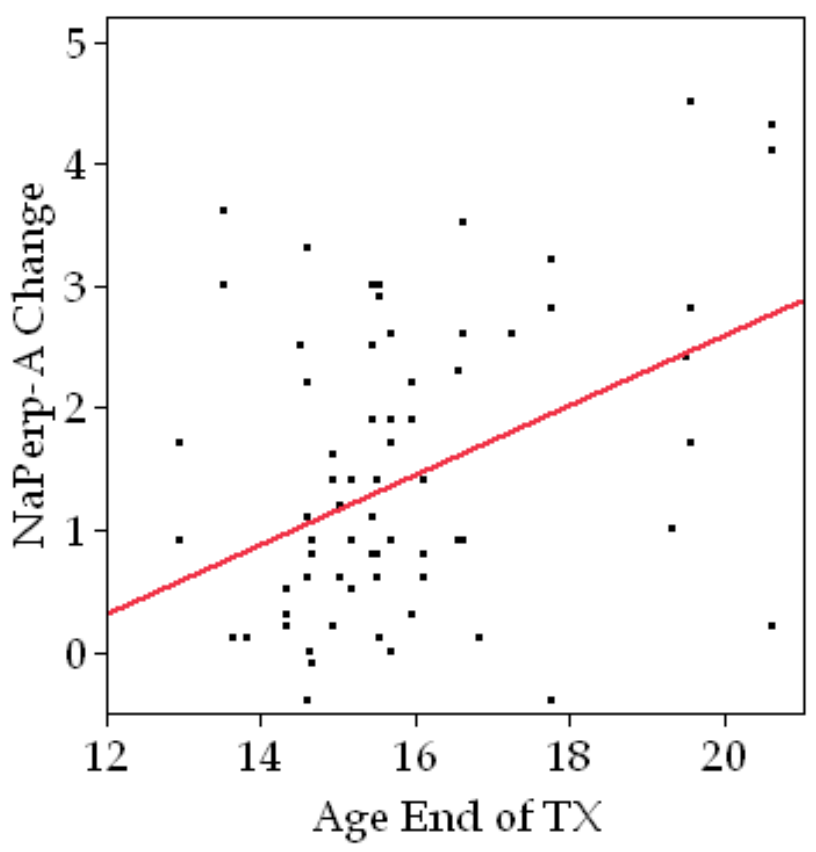

Figure 4-1. Plot of the subject's age at the end of treatment against the amount of posttreatment change in NaPerp to A point.

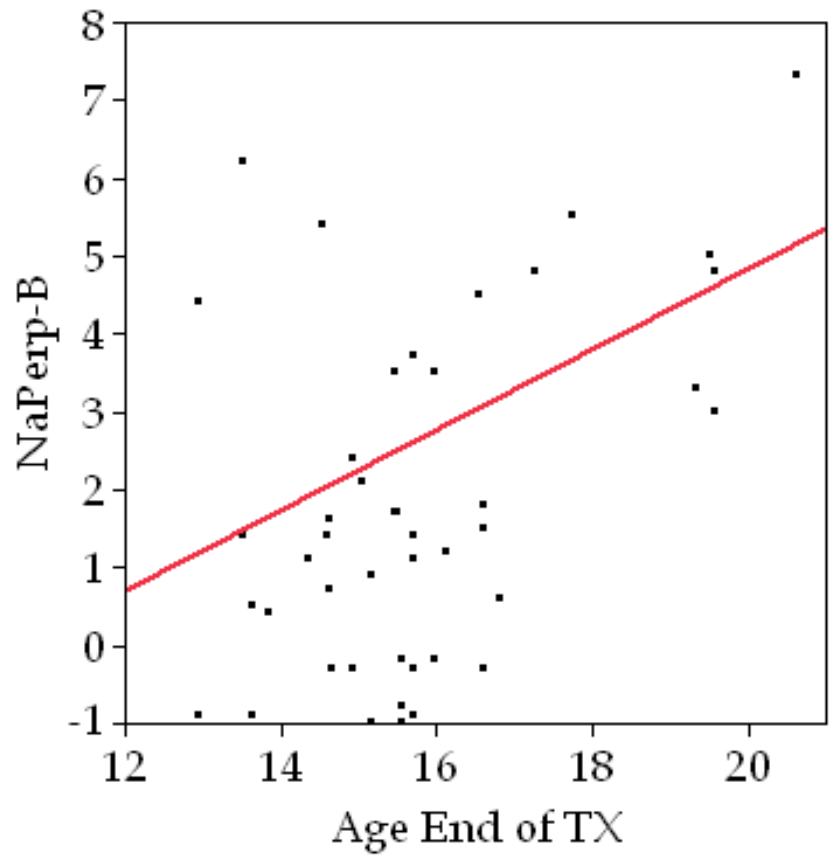

Figure 4-2. Plot of the subject's age at the end of treatment against the amount of posttreatment change in NaPerp to B point. 


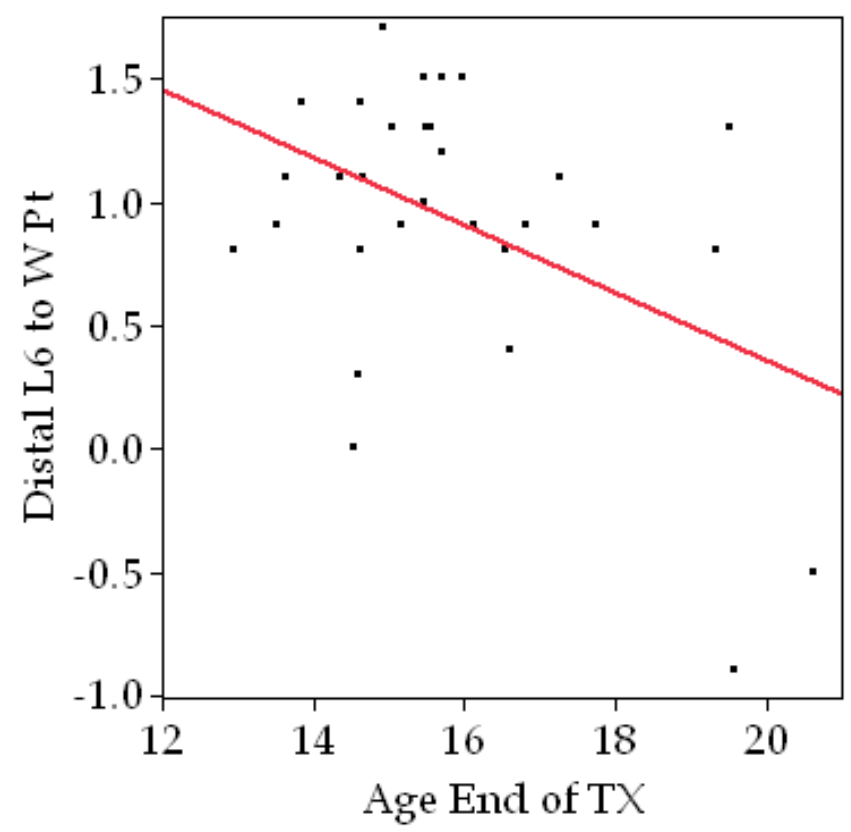

Figure 4-3. Plot of the subject's age at the end of treatment against the amount of posttreatment change in distal L6 to $\mathrm{W}$ point. 
the age at the end of treatment increases. In other words, this measure of arch length (and position of L6) increases as the first molar moves slightly to the distal relative to $\mathrm{W}$ point. (This analysis cannot distinguish between tipping and bodily movements of L6.) This change decreases with the subject's age, with a downward slope of $-0.14 \mathrm{~mm} /$ year.

The fourth significant variable is change in the Nasolabial Angle (Figure 4-4). Cases finished early in adolescence tend to exhibit an up-righting (increase) in the Nasolabial Angle, while those finished in the later teens tend to experience a decrease in the angle, which is a proclination of the upper lip to the labial. The regression coefficient is -1.94 degrees/year, meaning that the change diminishes about 2 degrees for every year added to age at the end of treatment. In other words, cases treated in the later teens tend to decrease their Nasolabial Angle the most following treatment.

\section{Duration of Treatment}

A shortcoming of the approach just discussed - that depends on the age at the end of treatment - is that there was no fixed time for the recall examination. Selection criterion was that a case be a minimum of 10 years out of treatment, but there was a range of times between end of treatment at the follow-up examination. Since we suppose that growth and the opportunity for posttreatment change are functions of the time out of treatment, not controlling for this source of variation probably weakens the veracity of these tests.

In this sample $(n=30)$, the range of years from the end of treatment to the recall examination is from 7.5 to 19.0 years, with a mean of 12.5 years (median $=$ 12.2 years). Table $4-2$ lists the statistical results of regressing the amount of posttreatment change on the duration of time (years) between the end of treatment and the recall examination. The equation is:

$$
\text { Post-TX change }=\mathrm{a}+\mathrm{b}(\text { duration before recall })
$$

where " $a$ " is the Y-intercept and " $b$ " is the regression coefficient.

Seven of the 59 variables exhibit a statistically significant association between the amount of change and the duration from posttreatment to the recall examination. These are (1) Sella-Nasion distance, (2) Sella-A distance, (3) L1 to $\mathrm{NB}^{\circ}$, (4) IMPA, (5) FMIA, and (6) incisor overbite, and (7) W point to soft tissue Pogonion'. 


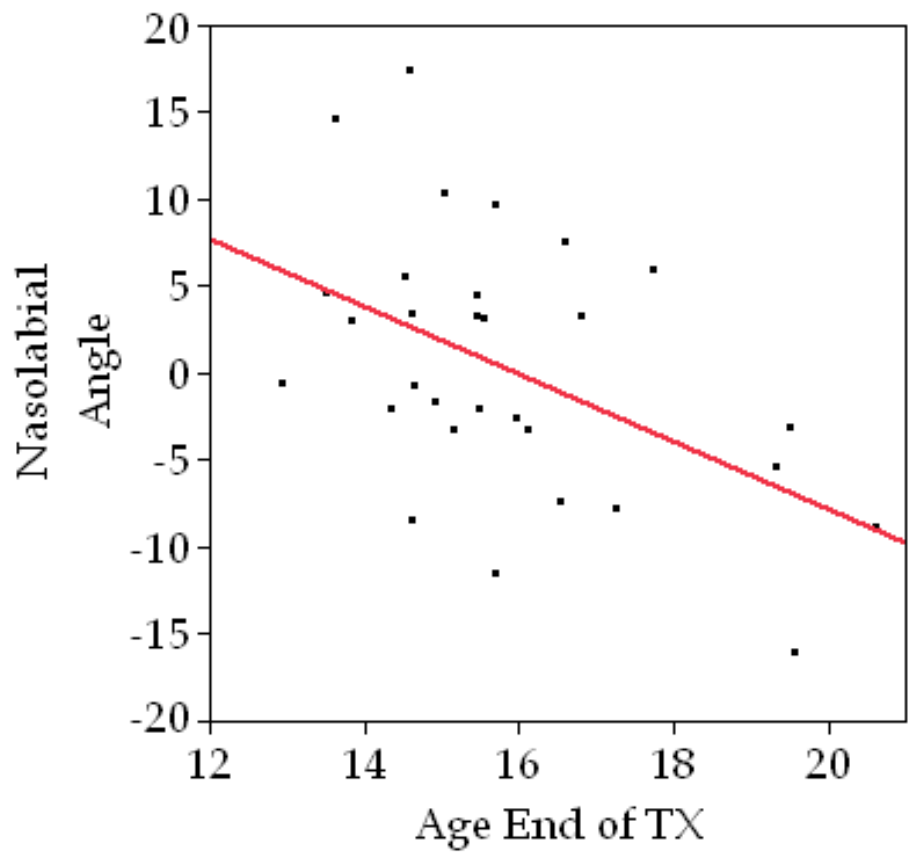

Figure 4-4. Plot of the subject's age at the end of treatment against the amount of posttreatment change in nasolabial angle. Most changes are negative, meaning that the angle gets smaller as the upper lip moves labially after treatment. 
Table 4-2. Results of linear regression analysis testing whether the duration of time following treatment until the recall examination is predictive of the amount of post-treatment change.

\begin{tabular}{|c|c|c|c|c|c|c|}
\hline \multirow[b]{2}{*}{ Variable } & \multicolumn{3}{|c|}{ Y-Intercept } & \multicolumn{3}{|c|}{ Regression Coefficient } \\
\hline & Estimate & T-Test & P-Value & Estimate & T-Test & P-Value \\
\hline Se-Na & 1.21 & 5.01 & $<0.0001$ & 0.12 & 6.39 & $\leq 0.0001$ \\
\hline Se-Ba & 2.74 & 1.58 & 0.1259 & -0.04 & -0.32 & 0.7532 \\
\hline $\mathrm{Na}-\mathrm{Ba}$ & 4.95 & 3.49 & 0.0016 & 0.05 & 0.41 & 0.6852 \\
\hline Na-Se-Ba & -1.02 & -0.60 & 0.5510 & 0.08 & 0.63 & 0.5329 \\
\hline Na-ANS & 2.26 & 2.17 & 0.0387 & 0.07 & 0.85 & 0.4029 \\
\hline Se-PNS & 1.34 & 0.77 & 0.4460 & 0.11 & 0.82 & 0.4169 \\
\hline Cd-A & 0.51 & 0.35 & 0.7315 & 0.24 & 2.12 & 0.0434 \\
\hline $\mathrm{Na}-\mathrm{PP}$ & 2.26 & 2.17 & 0.0387 & 0.07 & 0.85 & 0.4029 \\
\hline Me-PP & 2.03 & 1.27 & 0.2158 & 0.19 & 1.48 & 0.1499 \\
\hline $\mathrm{Na}-\mathrm{Me}$ & 4.28 & 2.61 & 0.0142 & 0.23 & 1.83 & 0.0782 \\
\hline Se-Go & 2.26 & 0.76 & 0.4512 & 0.15 & 0.65 & 0.5186 \\
\hline $\mathrm{PFH} / \mathrm{AFH}$ & 0.00 & -0.16 & 0.8776 & 0.00 & -0.07 & 0.9454 \\
\hline SNA & 0.33 & 2.33 & 0.0270 & 0.01 & 0.77 & 0.4504 \\
\hline NaPerp-A & 1.69 & 1.76 & 0.0896 & -0.02 & -0.30 & 0.7695 \\
\hline $\mathrm{PtV}-\mathrm{A}$ & 1.07 & 0.82 & 0.4176 & 0.13 & 1.28 & 0.2121 \\
\hline Se-A & 2.44 & 2.80 & 0.0091 & 0.15 & 2.25 & $\underline{0.0326}$ \\
\hline PP-SN & 1.58 & 1.05 & 0.3048 & -0.09 & -0.77 & 0.4494 \\
\hline SNB & 1.06 & 5.00 & $<0.0001$ & -0.02 & -1.41 & 0.1697 \\
\hline NaPerp-B & 3.43 & 2.01 & 0.0545 & -0.06 & -0.44 & 0.6623 \\
\hline PtV-B & 2.23 & 5.55 & $<0.0001$ & 0.02 & 0.54 & 0.5948 \\
\hline Cd-Gn & 5.26 & 3.36 & 0.0023 & 0.11 & 0.87 & 0.3928 \\
\hline Cd-Go & 4.97 & 2.01 & 0.0539 & 0.03 & 0.14 & 0.8890 \\
\hline Go-Pg & 1.58 & 0.69 & 0.4955 & 0.23 & 1.31 & 0.1998 \\
\hline Y-Axis & -1.82 & -1.72 & 0.0974 & 0.11 & 1.28 & 0.2125 \\
\hline NaPerp-Pg & 4.60 & 2.40 & 0.0233 & -0.21 & -1.39 & 0.1754 \\
\hline ANB & -0.61 & -2.67 & 0.0125 & 0.02 & 1.39 & 0.1765 \\
\hline AO-BO & -0.25 & -0.12 & 0.9032 & -0.02 & -0.12 & 0.9020 \\
\hline FMA & -1.01 & -0.52 & 0.6092 & 0.04 & 0.25 & 0.8050 \\
\hline FMA_b & -0.86 & -0.43 & 0.6711 & 0.03 & 0.18 & 0.8595 \\
\hline Na-A-Pg & -2.14 & -1.35 & 0.1884 & 0.21 & 1.69 & 0.1023 \\
\hline FH-Na-Pg & 2.91 & 2.50 & 0.0186 & -0.13 & -1.40 & 0.1715 \\
\hline U1/L1 & 11.48 & 2.24 & 0.0329 & -0.39 & -0.99 & 0.3323 \\
\hline U1/SN & 2.85 & 0.70 & 0.4900 & -0.48 & -1.50 & 0.1447 \\
\hline U1-NA ${ }^{\circ}$ & 2.41 & 0.60 & 0.5562 & -0.48 & -1.51 & 0.1422 \\
\hline U1-NA mm & 1.49 & 1.12 & 0.2712 & -0.14 & -1.33 & 0.1937 \\
\hline
\end{tabular}


Table 4-2. Continued.

\begin{tabular}{lrrrrrrr}
\hline & \multicolumn{3}{c}{ Y-Intercept } & & \multicolumn{3}{c}{ Regression Coefficient } \\
\cline { 2 - 4 } Variable & Estimate & T-Test & P-Value & & Estimate & T-Test & P-Value \\
\hline & & & & & & & \\
L1-NB & -13.14 & -3.19 & 0.0035 & & 0.82 & 2.55 & $\underline{\mathbf{0 . 0 1 6 3}}$ \\
L1-NB mm & -0.85 & -0.66 & 0.5139 & & -0.04 & -0.38 & 0.7078 \\
IMPA PostTx & -14.98 & -3.10 & 0.0044 & 0.87 & 2.30 & $\underline{\mathbf{0 . 0 2 9 3}}$ \\
FMIA & 15.65 & 3.43 & 0.0019 & -0.89 & -2.48 & $\underline{\mathbf{0 . 0 1 9 4}}$ \\
U1-APo & 1.27 & 1.37 & 0.1830 & -0.10 & -1.38 & 0.1789 \\
L1-APo & -0.90 & -0.86 & 0.3963 & & 0.00 & -0.03 & 0.9784 \\
D Occ Pl-FH & -5.09 & -2.04 & 0.0504 & & 0.26 & 1.33 & 0.1956 \\
Overbite & 2.42 & 3.84 & 0.0006 & -0.12 & -2.48 & $\underline{\mathbf{0 . 0 1 9 5}}$ \\
Overjet & 2.12 & 2.50 & 0.0186 & -0.09 & -1.42 & 0.1665 \\
L1-W Pt & -1.76 & -2.19 & 0.0372 & & 0.14 & 2.18 & 0.0381 \\
Distal L6-W Pt & -0.60 & -1.25 & 0.2201 & & -0.03 & -0.70 & 0.4902 \\
U1-PtV & 4.52 & 2.97 & 0.0060 & & -0.13 & -1.06 & 0.2984 \\
U6-PtV & 3.40 & 2.36 & 0.0258 & & -0.08 & -0.74 & 0.4661 \\
Distal L6-L1 & 1.10 & 2.32 & 0.0281 & & 0.01 & 0.32 & 0.7528 \\
Distal U6-U1 & 1.07 & 2.01 & 0.0547 & & 0.05 & 1.27 & 0.2150 \\
Z Angle & 6.13 & 1.79 & 0.0846 & & -0.13 & -0.49 & 0.6303 \\
E Plane-Ls & -0.17 & -0.21 & 0.8368 & & -0.04 & -0.65 & 0.5197 \\
E Plane-Li & -1.15 & -1.18 & 0.2494 & & 0.00 & -0.05 & 0.9628 \\
Nasolabial & & & & & & \\
Angle & -9.45 & -1.57 & 0.1268 & 0.78 & 1.67 & 0.1066 \\
Li-A'-Pg' & -1.61 & -1.23 & 0.2307 & -0.01 & -0.08 & 0.9391 \\
Sn-StoS & 2.78 & 2.36 & 0.0256 & -0.09 & -0.95 & 0.3495 \\
Sn-Ls & 2.10 & 6.14 & $<0.0001$ & -0.03 & -1.08 & 0.2901 \\
Sn-Li & 2.15 & 1.17 & 0.2519 & 0.01 & 0.06 & 0.9563 \\
NaPerp-Pr & 2.21 & 4.22 & 0.0002 & -0.03 & -0.64 & 0.5262 \\
W Pt-Pg' & 0.50 & 1.08 & 0.2885 & 0.08 & 2.33 & $\underline{\mathbf{0 . 0 2 7 5}}$ \\
\hline
\end{tabular}

Note: Statistical significant regression coefficients are underlined. 
Results for Sella-Nasion $(\mathrm{P}<0.0001)$ are readily interpreted: The longer the time from the end of treatment until the recall examination, the greater the observed growth in this dimension (Figure 4-5). The regression equation is $\mathrm{Y}=$ $1.21+0.12$ (Years), so the Sella-Nasion distance increased $1.21 \mathrm{~mm}$ plus 0.12 times the years after treatment. The average increase following treatment was 0.12 $\mathrm{mm}$ /year or about $1 \mathrm{~mm}$ in a decade. This is a modest change, but the change is surprisingly systematic across this sample of females $\left(r^{2}=59 \%\right)$.

The Sella-A distance also is significantly associated with duration to the recall examination $(P=0.0326)$, but the association is not as close as with SellaNasion (Figure 4-6). Here, the regression coefficient is positive $(b=0.15$ $\mathrm{mm}$ /year); on average, Sella-A grew forward $0.15 \mathrm{~mm} /$ year after the end of treatment, which is $1.5 \mathrm{~mm}$ for a decade of growth $\left(\mathrm{r}^{2}=15 \%\right)$. The full regression equation is $Y=2.44+0.15$ (Years), meaning-more correctly - that the average case increased $2.44 \mathrm{~mm}$ plus $0.15 \mathrm{~mm}$ times the number of years out of treatment.

The next significant association in Table 4-2 involves the mandibular central incisor (L1) angulation to the Na-B line. This association is positive, and the regression coefficient is 0.82 . Of interest (Figure 4-7), the $Y$ axis includes both negative and positive values. Some cases exhibited an increase (proclination) of the central incisor, but the majority of cases up-righted with time, and the longer the duration the less the net change. It may be that the short-term changes (relapse) differ in direction from the long-term aging process. The regression coefficient is 0.82 degrees per year.

IMPA also changed systematically with time out of treatment (Figure 4-8). The regression coefficient is 0.87 degrees/year. Since the majority of the changes are negative, the trend is for IMPA to upright in the majority of cases - just a discussed above for the L1-NB angle. In concert with the findings for the L1-NB angle, the largest changes were in subjects followed for the shortest intervals. Again, it may be that that the short-term response is for incisor up-righting, but the long-term net change is close to zero.

FMIA also changed systematically following treatment $(\mathrm{P}=00194)$. The trend (Figure 4-9) is for the lower incisor to increase over the short term, but then exhibit little net change when examined at the longer term ( $c a .15$ years). Once again, these findings are consistent with two complementary effects: First, over the short-term, orthodontic relapse (Horowitz and Hixon 1969) causes the incisor to upright. Then, over the long-term, proclination due to normative aging reverses the trend such that, in the cases followed longest, there is no net effect compared to the end of treatment. 


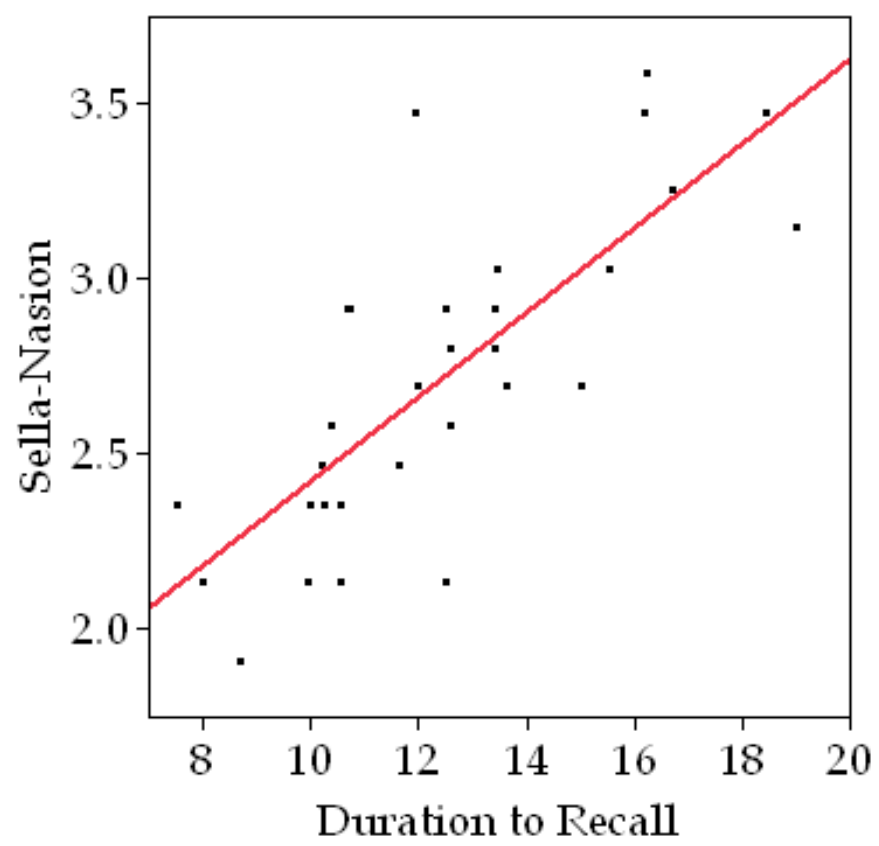

Figure 4-5. Plot of the subject's duration to the recall examination against the amount of posttreatment change (increase) in Sella-Nasion distance.

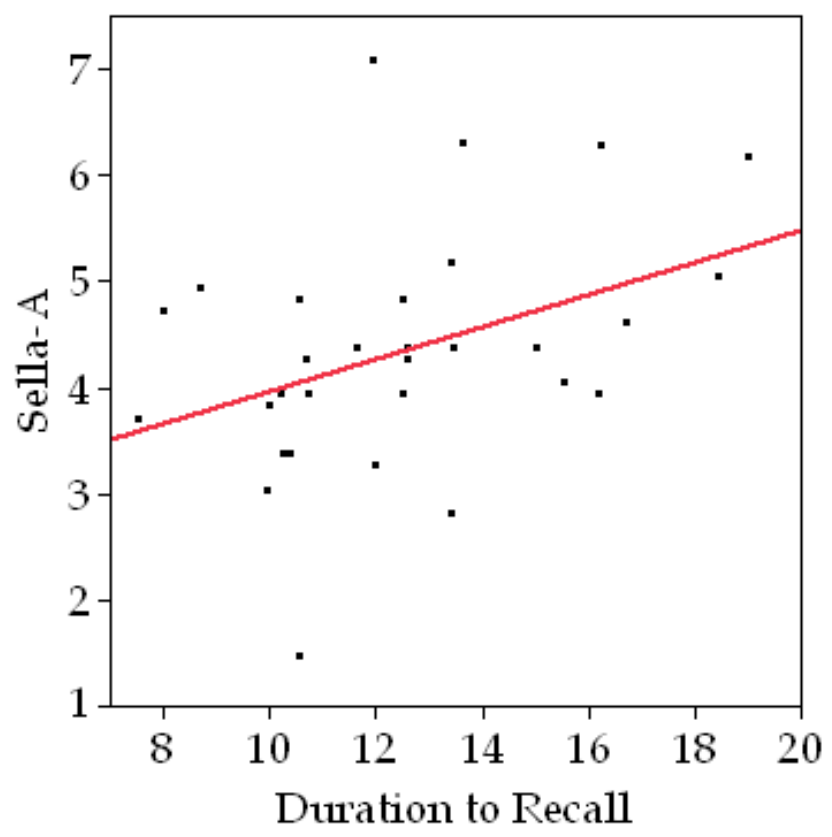

Figure 4-6. Plot of the subject's duration to the recall examination against the amount of posttreatment change in Sella-A distance. 


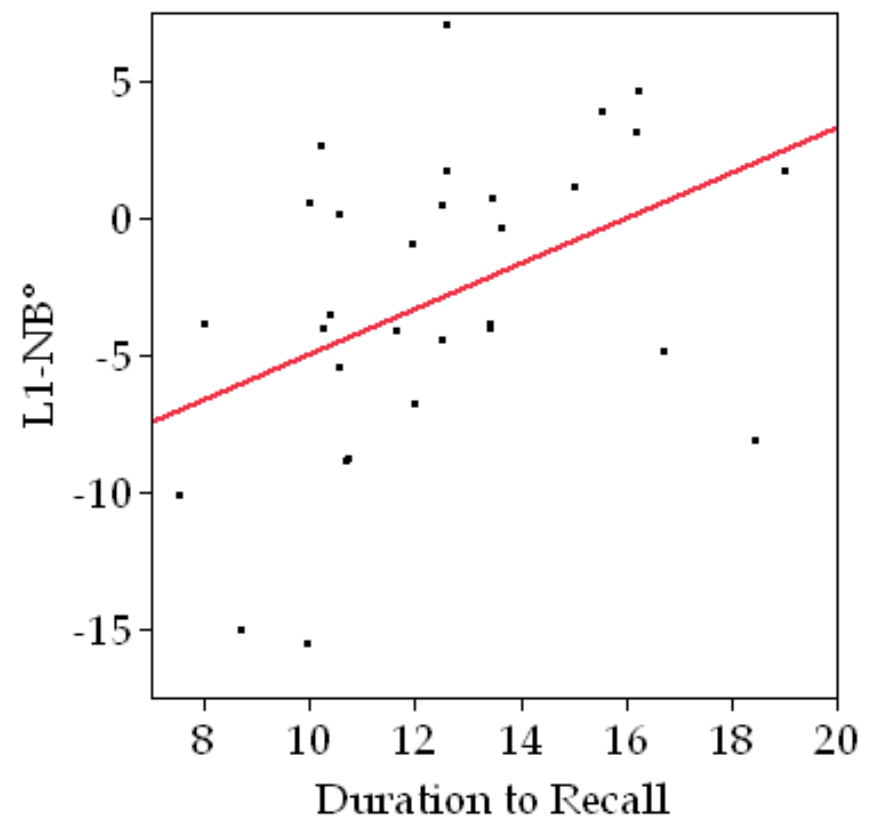

Figure 4-7. Plot of the subject's duration to the recall examination against the amount of posttreatment change in L1-NB angle.

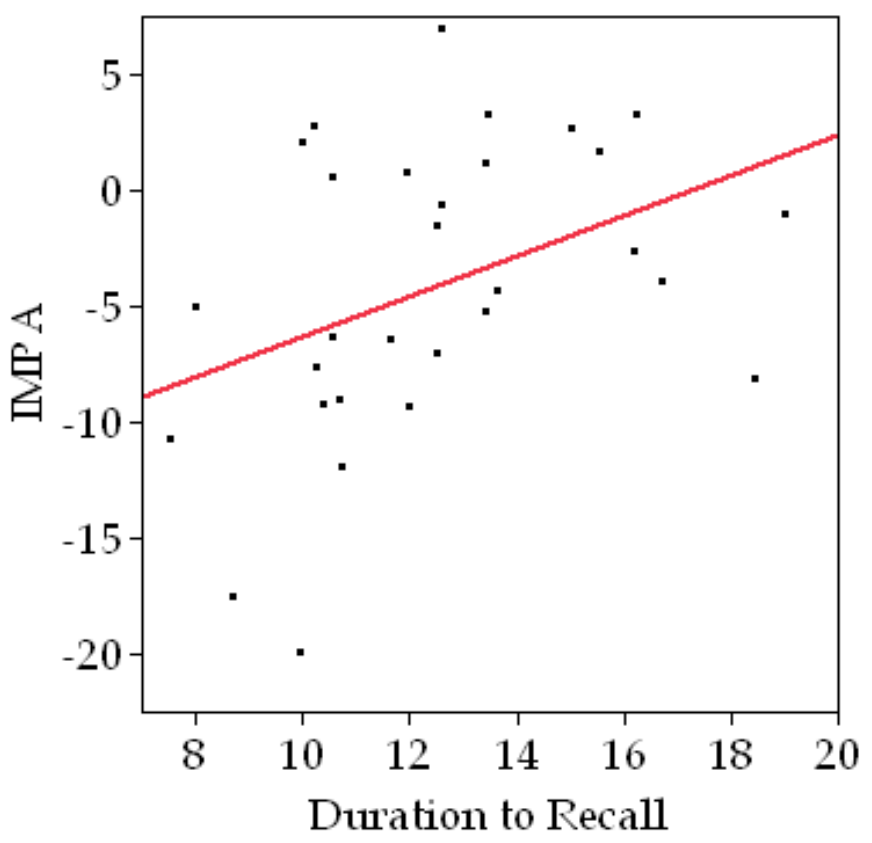

Figure 4-8. Plot of the subject's duration to the recall examination against the amount of posttreatment change in IMPA. 


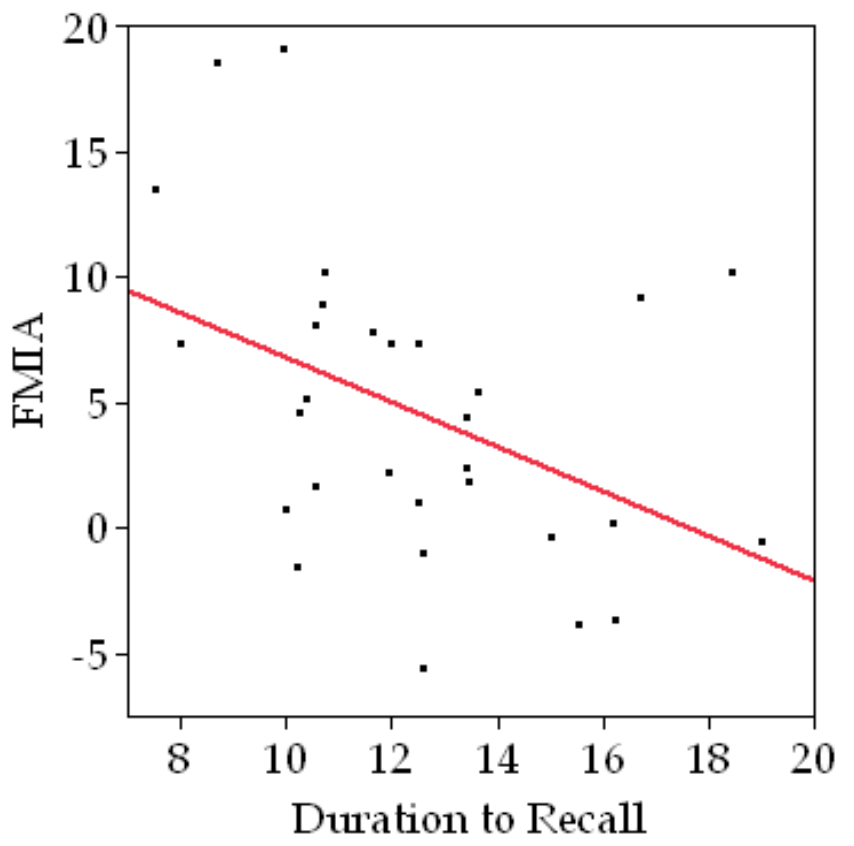

Figure 4-9. Plot of the subject's duration to the recall examination against the amount of posttreatment change in FMIA. 
Overbite changed in tandem with the time out of treatment (Table 4-2). The change is subtle (regression coefficient $=-0.12 \mathrm{~mm} /$ year), but the association is patterned across the cases $(\mathrm{P}=0.0195)$. The trend (Figure 4-10) is negative in that the amount of change - typically a deepening-diminishes with time out of treatment.

\section{Age at Treatment Controlling for Duration}

The prior two sections, while informative, both suffer from the limitation that duration of time till recall is not controlled (in the first section) or the age at treatment is not controlled (in the second section). In fact, while this lack of control is conceptually important, it will be seen that in no case did duration of time to the recall examination have a significant influence on the results. In this section we present the results of using analysis of covariance, where an association is sought between the amount of posttreatment change as a function of age at the end of treatment, while controlling for the duration to the recall examination (Table 4-3). The focus in on which variables are statistically associated with age at the end of treatment, and five of the 59 variables achieved significance.

Presentation does require some modification because curvilinear surface of the best fit regression in two planes of space cannot be printed. Instead, (1) the ANCOVA results are presented to confirm the statistical association, and then (2) duration of treatment is standardized by dividing the posttreatment change by the years out of treatment (and then this rate is plotted against age).

Nasion-Perpendicular to A point is one such variable. ANCOVA results are shown in Table 4-4, where age at the end of treatment is associated with the amount of posttreament change. The probability values associated with the $t$ tests show that (1) the Y-intercept is not significantly different from zero, (2) the duration-to-recall is not significant, and (3) the interaction effect likewise is insignificant. The key issue is that the age at the end of treatment is significantly associated with posttreatment growth of A point.

The slope of the regression line is $0.28 \mathrm{~mm} /$ year, which translates into 2.8 $\mathrm{mm}$ per decade, and the graph (Figure 4-11) shows that the response is fairly consistent throughout the sample. Indeed, this duration of treatment accounts for about a quarter of the variance $\left(\mathrm{r}^{2}=0.22\right)$.

The next significant association is for the U1/SN angle. The association with posttreatment change in U1/SN is marginally significant with age at end of treatment $(\mathrm{P}=0.0419)$ as shown in Table 4-5. The trend (Figure 4-12) is for the 


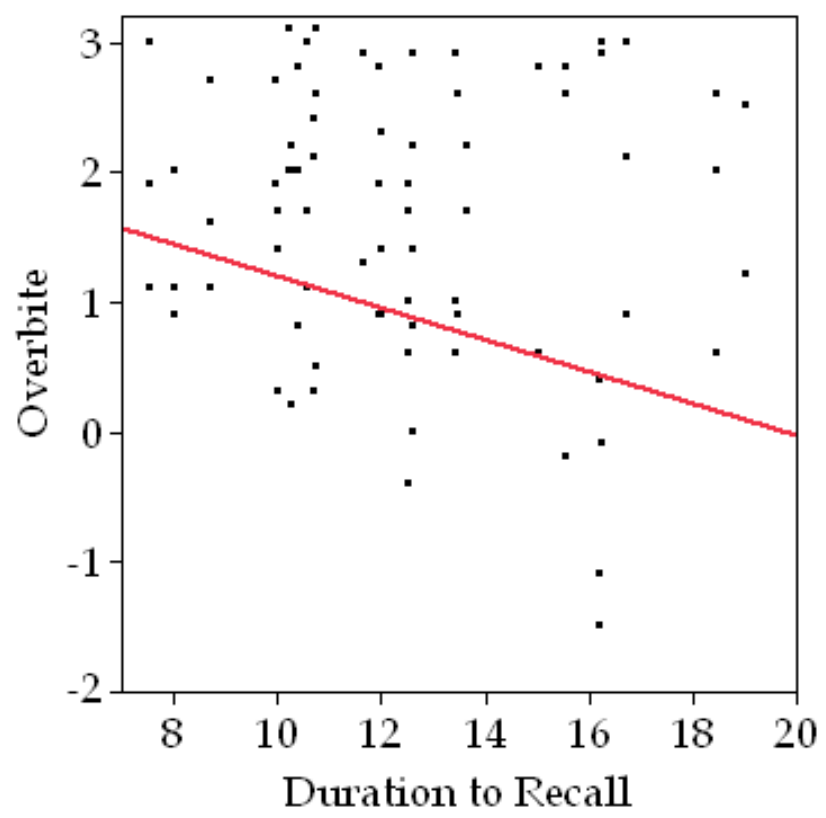

Figure 4-10. Plot of the subject's duration to the recall examination against the amount of posttreatment change in overbite. 
Table 4-3. Results of analysis of covariance testing for a statistical dependence between the subject's age at the end of treatment and the amount of posttreatment change with the duration of the recall examination as the covariate.

\begin{tabular}{lccccccc}
\hline & \multicolumn{2}{c}{ Age at End } & \multicolumn{2}{c}{ Duration } & \multicolumn{2}{c}{ Interaction } \\
Variable & $\mathrm{F}$ & $\mathrm{P}$ & $\mathrm{F}$ & $\mathrm{P}$ & $\mathrm{F}$ & $\mathrm{P}$ \\
\hline Se-Na & & & & & & & \\
Se-Ba & 0.24 & 0.6292 & 40.75 & $\underline{\mathbf{0 . 0 0 0 1}}$ & 1.43 & 0.2428 \\
Na-Ba & 1.97 & 0.6505 & 0.68 & 0.4175 & 8.09 & $\underline{\mathbf{0 . 0 0 8 6}}$ \\
Na-Se-Ba & 2.10 & 0.1590 & 0.19 & 0.6647 & 0.32 & 0.5781 \\
Na-ANS & 0.11 & 0.7480 & 0.27 & 0.8637 & 0.6089 & 2.09 & 0.0904 \\
Se-PNS & 0.01 & 0.9125 & 0.18 & 0.6723 & 4.71 & 0.1393 \\
Cd-A & 2.97 & 0.0967 & 5.41 & $\underline{\mathbf{0 . 0 2 8 1}}$ & 0.0393 & 0.7687 \\
Na-PP & 0.11 & 0.7480 & 0.27 & 0.6089 & 2.33 & 0.1393 \\
Me-PP & 2.02 & 0.1668 & 3.59 & 0.0693 & 2.06 & 0.1631 \\
Na-Me & 3.78 & 0.0626 & 5.03 & $\underline{\mathbf{0 . 0 3 3 7}}$ & 1.03 & 0.3190 \\
Se-Go & 0.12 & 0.7308 & 0.20 & 0.6587 & 0.57 & 0.4562 \\
PFH/AFH & 0.83 & 0.3710 & 0.11 & 0.7395 & 0.92 & 0.3458 \\
SNA & 1.79 & 0.1929 & 0.18 & 0.6785 & 1.20 & 0.2839 \\
NaPerp-A & 7.00 & $\underline{\mathbf{0 . 0 1 3 7}}$ & 0.01 & 0.9178 & 0.08 & 0.7768 \\
PtV-A & 0.22 & 0.6420 & 1.23 & 0.2785 & 0.08 & 0.7783 \\
Se-A & 0.97 & 0.3341 & 4.42 & $\underline{\mathbf{0 . 0 4 5 4}}$ & 1.28 & 0.2684 \\
PP-SN & 0.20 & 0.6583 & 0.41 & 0.5294 & 0.05 & 0.8219 \\
SNB & 2.26 & 0.1445 & 2.00 & 0.1696 & 0.56 & 0.4610 \\
NaPerp-B & 7.42 & 0.0114 & 0.04 & 0.8478 & 0.00 & 0.9773 \\
PtV-B & 0.75 & 0.3931 & 0.53 & 0.4729 & 0.46 & 0.5054 \\
Cd-Gn & 1.54 & 0.2251 & 1.17 & 0.2884 & 0.39 & 0.5360 \\
Cd-Gn_b & 1.51 & 0.2302 & 1.08 & 0.3081 & 0.38 & 0.5446 \\
Cd-Go & 0.09 & 0.7678 & 0.00 & 0.9555 & 0.33 & 0.5697 \\
Go-Pg & 0.10 & 0.7504 & 1.57 & 0.2209 & 0.01 & 0.9372 \\
Y-Axis & 0.86 & 0.3632 & 1.81 & 0.1902 & 1.04 & 0.3162 \\
NaPerp-Pg & 1.22 & 0.2804 & 1.97 & 0.1725 & 0.68 & 0.4159 \\
ANB & 0.28 & 0.6016 & 1.30 & 0.2649 & 2.39 & 0.1346 \\
AO-BO & 0.29 & 0.5925 & 0.04 & 0.8506 & 0.01 & 0.9207 \\
FMA & 0.00 & 0.9874 & 0.26 & 0.6162 & 2.00 & 0.1693 \\
Na-A-Pg & 0.07 & 0.7896 & 2.56 & 0.1218 & 0.01 & 0.9262 \\
FH-Na-Pg & 1.69 & 0.2052 & 1.91 & 0.1786 & 0.51 & 0.4815 \\
U1/L1 & 0.62 & 0.4371 & 1.02 & 0.3211 & 0.56 & 0.4593 \\
U1/SN & 4.58 & $\underline{\mathbf{0 . 0 4 1 9}}$ & 2.83 & 0.1047 & 0.15 & 0.7038 \\
U1-NA & 4.47 & $\underline{\mathbf{0 . 0 4 4 2}}$ & 2.82 & 0.1050 & 0.18 & 0.6739 \\
& & & & & &
\end{tabular}


Table 4-3. Continued.

\begin{tabular}{lccccccc}
\hline & \multicolumn{2}{c}{ Age at End } & \multicolumn{2}{c}{ Duration } & \multicolumn{2}{c}{ Interaction } \\
\cline { 2 - 5 } Variable & $\mathrm{F}$ & $\mathrm{P}$ & $\mathrm{F}$ & $\mathrm{P}$ & $\mathrm{F}$ & $\mathrm{P}$ \\
\hline U1-NA mm & 1.68 & 0.2060 & 2.84 & 0.1042 & 1.58 & 0.2194 \\
L1-NB $^{\circ}$ & 1.17 & 0.2891 & 7.24 & $\underline{\mathbf{0 . 0 1 2 3}}$ & 0.39 & 0.5355 \\
L1-NB mm & 0.14 & 0.7120 & 0.29 & 0.5968 & 0.55 & 0.4637 \\
IMPA PostTx & 0.08 & 0.7821 & 4.77 & $\underline{\mathbf{0 . 0 3 8 2}}$ & 0.00 & 0.9703 \\
FMIA & 0.10 & 0.7604 & 6.14 & $\underline{\mathbf{0 . 0 2 0 0}}$ & 0.29 & 0.5965 \\
U1-APo & 0.15 & 0.6983 & 2.21 & 0.1489 & 0.54 & 0.4687 \\
L1-APo & 0.03 & 0.8753 & 0.01 & 0.9440 & 0.03 & 0.8754 \\
D Occ Pl-FH & 0.27 & 0.6053 & 1.82 & 0.1887 & 0.06 & 0.8149 \\
Overbite & 0.10 & 0.7581 & 7.77 & $\underline{\mathbf{0 . 0 0 9 8}}$ & 2.74 & 0.1098 \\
Overjet & 0.20 & 0.6612 & 2.43 & 0.1309 & 0.73 & 0.4007 \\
L1-W Pt & 0.11 & 0.7426 & 3.27 & 0.0821 & 2.91 & 0.0999 \\
Distal L6-W Pt & 5.94 & $\underline{\mathbf{0 . 0 2 1 9}}$ & 0.74 & 0.3984 & 3.10 & 0.0901 \\
U1-PtV & 0.95 & 0.3379 & 1.20 & 0.2830 & 0.76 & 0.3918 \\
U6-PtV & 0.01 & 0.9268 & 0.53 & 0.4722 & 0.05 & 0.8251 \\
Distal L6-L1 & 1.93 & 0.1761 & 0.01 & 0.9398 & 0.27 & 0.6110 \\
Distal U6-U1 & 0.07 & 0.7917 & 1.13 & 0.2976 & 0.26 & 0.6121 \\
Z Angle & 0.08 & 0.7793 & 0.26 & 0.6140 & 0.18 & 0.6749 \\
E Plane-Ls & 1.92 & 0.1777 & 0.15 & 0.7003 & 0.35 & 0.5597 \\
E Plane-Li & 0.19 & 0.6679 & 0.03 & 0.8539 & 1.02 & 0.3213 \\
Nasolabial & & & & & & \\
Angle & 7.51 & $\underline{\mathbf{0 . 0 1 0 9}}$ & 2.76 & 0.1087 & 0.48 & 0.4937 \\
Li-A'-Pg' & 0.16 & 0.6900 & 0.03 & 0.8717 & 0.49 & 0.4907 \\
Sn-StoS & 0.73 & 0.3993 & 0.69 & 0.4143 & 0.96 & 0.3352 \\
Sn-Ls & 0.03 & 0.8670 & 1.17 & 0.2901 & 0.07 & 0.7994 \\
Sn-Li & 0.99 & 0.3281 & 0.01 & 0.9357 & 0.47 & 0.4991 \\
NaPerp-Pr & 0.31 & 0.5813 & 0.69 & 0.4131 & 0.76 & 0.3922 \\
W Pt-Pg' & 2.81 & 0.1056 & 4.62 & 0.0411 & 0.00 & 0.9474 \\
\hline
\end{tabular}

Notes: Statistical significant regression coefficients are underlined. F, f-value. P, p-value. 
Table 4-4. Results of ANCOVA for nasion-perpendicular to A point.

\begin{tabular}{lcccc}
\hline \multicolumn{1}{c}{ Term } & Estimate & SEM & T-Test & P-Value \\
\hline Intercept & -2.96 & 2.00 & -1.48 & 0.1512 \\
End age & 0.28 & 0.11 & 2.64 & 0.0137 \\
Duration & -0.01 & 0.07 & -0.10 & 0.9178 \\
Interaction & -0.01 & 0.03 & -0.29 & 0.7768 \\
\hline
\end{tabular}

Notes: There is $1 \mathrm{df}$ associated with each test. SEM, standard error of mean.

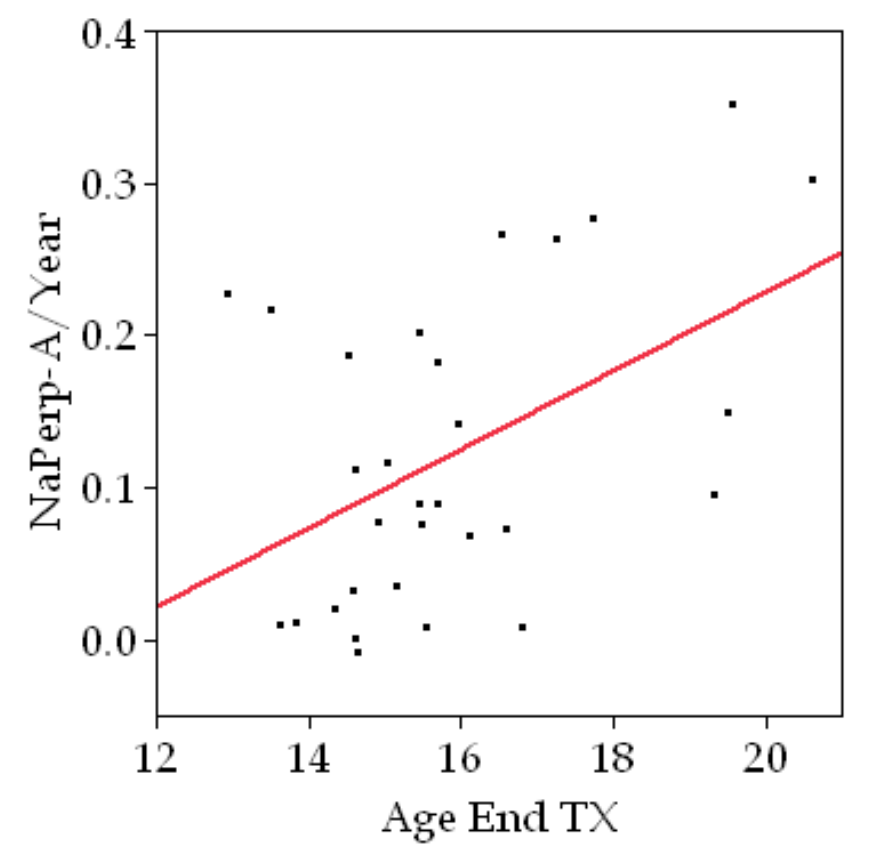

Figure 4-11. Association between the subject's age at the end of treatment against the amount of posttreatment change in nasion-perpendicular to A point. 
Table 4-5. Results of ANCOVA for nasion-perpendicular to A point.

\begin{tabular}{lcccc}
\hline \multicolumn{1}{c}{ Term } & Estimate & SEM & T-Test & P-Value \\
\hline Intercept & 19.28 & 8.78 & 2.19 & 0.0373 \\
End age & -1.00 & 0.47 & -2.14 & 0.0419 \\
Duration & -0.52 & 0.31 & -1.68 & 0.1047 \\
Interaction & 0.06 & 0.15 & 0.38 & 0.7038 \\
\hline
\end{tabular}

Notes: There is $1 \mathrm{df}$ associated with each test. SEM, standard error of mean.

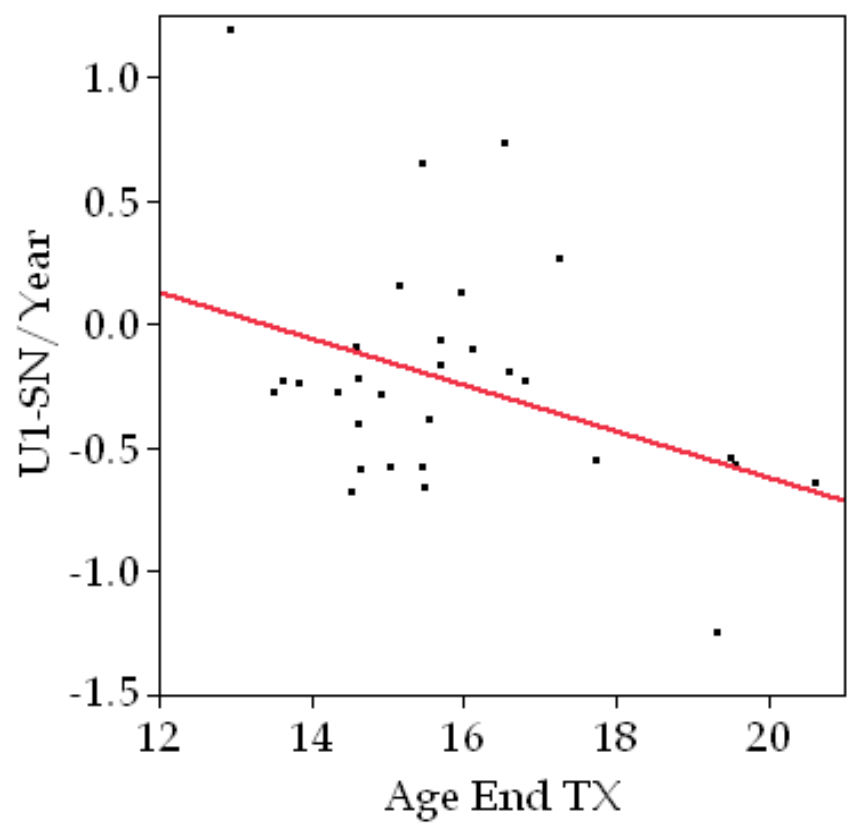

Figure 4-12. Association between the subject's age at the end of treatment against the amount of posttreatment change in the angle U1 to Sella-Nasion. 
upper incisor to upright over time (i.e., the angle gets smaller). The change is small (regression coefficient $=-0.09$ degrees $/$ year), but the trend explains $\left(\mathrm{r}^{2}\right)$ about $14 \%$ of the variance in the change in this angle.

The complementary variable $\left(\mathrm{U}_{\left.1-\mathrm{NA}^{\circ}\right)}\right.$ also achieves statistical significance (Table 4-6). The trend (Figure 4-13) is for the upper incisor to become progressively more upright with age following treatment. The change is subtle, only 0.09 degrees per year (or about 1 degree per decade), but, statistically, the trend with time explains $\left(\mathrm{r}^{2}\right) 14 \%$ percent of the variation.

The distance of L6 mesial to the $\mathrm{W}$ point (on the lingual of the mandibular symphysis) also changed systematically $(\mathrm{P}=0.0219$; Table $4-7)$. Changes are small in these nonextraction cases, but the overall trend is for this measure of arch length to increase after treatment. The specific question asked here is whether this amount of increase is predicted by the subject's age at the end of treatment (Table 4-7), and the answer is yes, it is. As graphed in Figure 4-14, those finishing treatment at the earliest ages have the highest rates of increase, and those finishing late in adolescence experience the slowest (near-zero) changes. Indeed, in the cases treated at the oldest ages in this sample (around 20 years), the posttreatment changes actually are negative (i.e., decreases in arch length).

The fifth and final significant association identified in Table 4-3 is between the subject's age at the end of treatment and change in the Nasolabial Angle (Table 4-8). Most cases in this sample experienced an increase in this angle following treatment, meaning that the upper lip becomes more upright. The question here is whether the amount of change following treatment is predicted by the age at which treatment is completed. The association is significant $(\mathrm{P}=$ 0.0109). The trend (Figure 4-15) for those treated at the earliest ages to experience almost no change, while as age at the end of treatment increases, the amount of uprighting also increases. The regression coefficient is -1.79 per year of age.

\section{Prediction Overview}

In concept, the ANCOVA results are the most reliable of three approaches used here to predict the amount of change following orthodontic treatment. On the other hand, in practice, the duration to the recall examination did not contribute significantly to any of the models, evidently because of the large interindividual variations among the subjects. 
Table 4-6. Results of ANCOVA for U1 to NA .

\begin{tabular}{lcccc}
\hline \multicolumn{1}{c}{ Term } & Estimate & SEM & T-Test & P-Value \\
\hline Intercept & 18.55 & 8.75 & 2.12 & 0.0437 \\
End age & -0.98 & 0.47 & -2.11 & 0.0442 \\
Duration & -0.52 & 0.31 & -1.68 & 0.1050 \\
Interaction & 0.06 & 0.15 & 0.43 & 0.6739 \\
\hline
\end{tabular}

Notes: There is $1 \mathrm{df}$ associated with each test. SEM, standard error of mean.

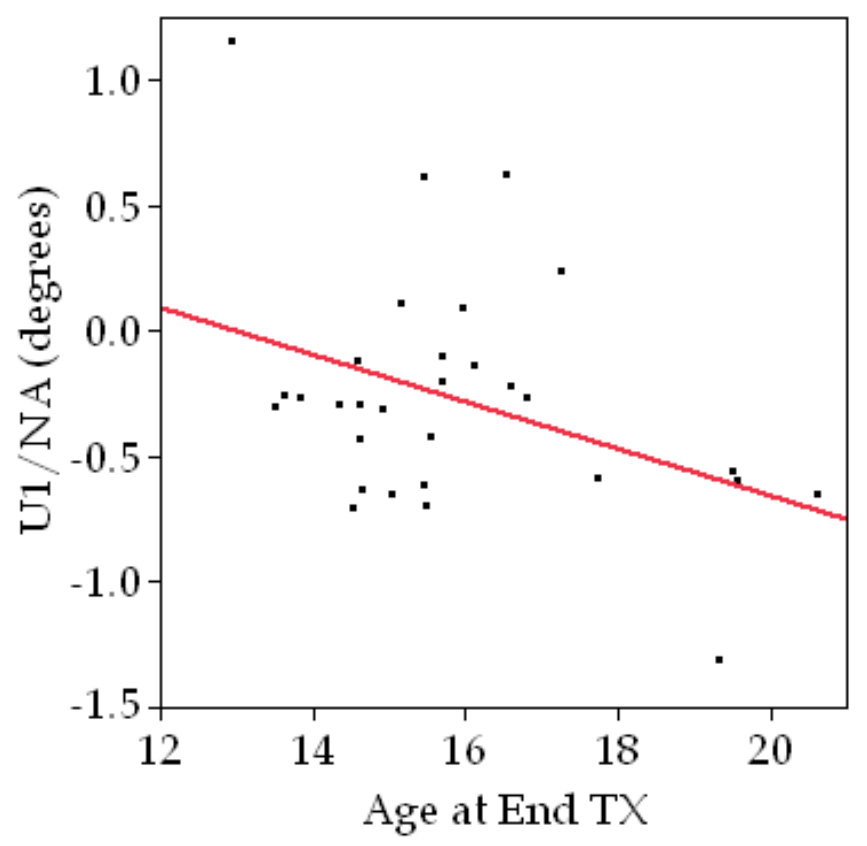

Figure 4-13. Association between the subject's age at the end of treatment against the amount of posttreatment change in the angle U1 to NA. 
Table 4-7. Results of ANCOVA for L6 to W point.

\begin{tabular}{lcccc}
\hline \multicolumn{1}{c}{ Term } & Estimate & SEM & T-Test & P-Value \\
\hline Intercept & 2.55 & 0.96 & 2.66 & 0.0131 \\
End age & -0.12 & 0.05 & -2.44 & 0.0219 \\
Duration & 0.03 & 0.03 & 0.86 & 0.3984 \\
Interaction & 0.03 & 0.02 & 1.76 & 0.0901 \\
\hline
\end{tabular}

Notes: There is $1 \mathrm{df}$ associated with each test. SEM, standard error of mean.

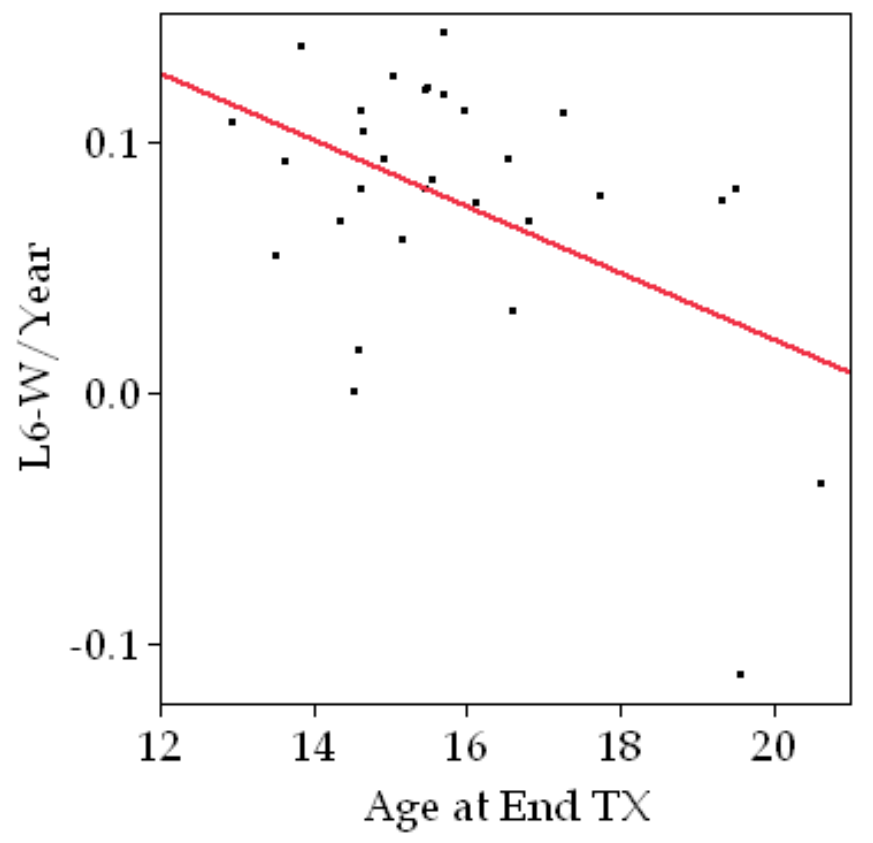

Figure 4-14. Association between the subject's age at the end of treatment against the amount of posttreatment change in the distance L6 to W Point. 
Table 4-8. Results of ANCOVA for nasolabial angle.

\begin{tabular}{lcccc}
\hline \multicolumn{1}{c}{ Term } & Estimate & SEM & T-Test & P-Value \\
\hline Intercept & 19.76 & 12.27 & 1.61 & 0.1195 \\
End age & -1.79 & 0.65 & -2.74 & 0.0109 \\
Duration & 0.72 & 0.43 & 1.66 & 0.1087 \\
Interaction & 0.15 & 0.21 & 0.69 & 0.4937 \\
\hline
\end{tabular}

Notes: There is $1 \mathrm{df}$ associated with each test. SEM, standard error of mean.

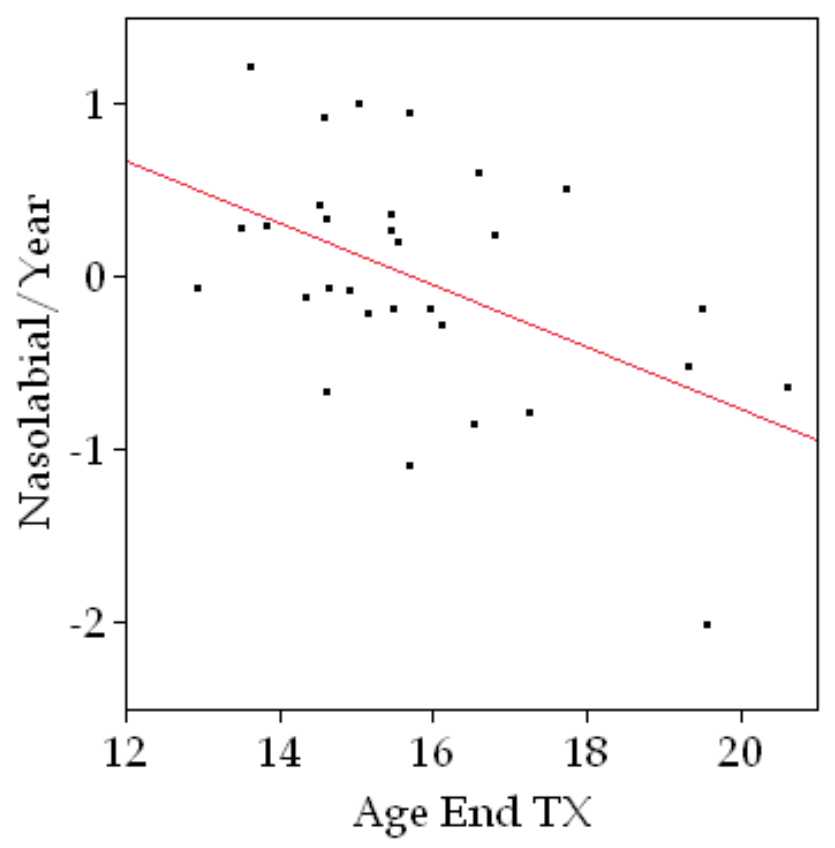

Figure 4-15. Association between the subject's age at the end of treatment against the amount of posttreatment change in the nasolabial angle. 
Perhaps the major finding in these results is that most of the variables have no statistically significant association with either the time of treatment or the duration of time until the recall examination. Again, the evident cause is the considerable subject-by-subject differences in how people change after orthodontic treatment. All of these cases are women. Since men grow more, and grow for a longer interval of time, it is supposed that the predictability of posttreatment changes would be different if a cohort of men were studied.

A salient point in these prediction equations is that - in addition to identifying the nature of the posttreatment change-it also matters when (how old) the subject is at treatment, since this too determines the rate of change. All of these cases were in the 12-to-20 age range at the end of treatment, so it is not as if we were comparing adolescents with older adults. These systematic differences are occurring within the "teenage" years. 


\section{CHAPTER 5. DISCUSSION}

As this and previous studies reveal, post-adolescent growth following treatment has proven to be a significant factor in the amount of overall postadolescent change (Behrents 1985; Driscoll-Gilliland et al. 2001). Although orthodontic treatment is intended to create more "ideal" functional relationships in the short-term, maturational changes within the craniofacial skeleton continue to influence these relationships long after treatment has been concluded. Because posttreatment relationships are not natural relationships developed from a normal, unaltered growth process, there is also the potential for posttreatment change to be influenced by relapse towards the original biological equilibrium (Proffit 1978; Ormiston et al. 2005).

In the present study, the average age at the end of treatment was 16 years, and the average age at recall was 28 years. As such, the interval of measurable posttreatment change occurred between late adolescence and early adulthood. Sinclair and Little (1985) found significant changes for most cephalometric variables in the seven-year period immediately following the peak in adolescent growth in untreated subjects. Behrents (1985) found that the craniofacial complex continues to experience significant change from late adolescence through late adulthood in untreated subjects, although the changes become smaller and more gradual over time. Anticipated posttreatment changes include a general enlargement of the skull, downward and forward growth of the maxilla and mandible, uprighting of the maxillary and mandibular incisors, forward growth of the soft tissue nose and chin, and a flattening of the facial profile. As previous studies have shown (Harris, Gardner and Vaden 1999), most of the growth-related change in the current study is believed to have occurred soon after treatment (i.e. late adolescent growth), which means that the growth rate was not likely to be linear with time.

The following is a discussion analyzing the posttreatment cephalometric changes found for a sample of 30 Caucasian American females, all of whom presented with a Class II division 1 malocclusion at the pretreatment examination. All subjects were treated orthodontically without extractions in the private practice of Dr. Richard A. Williams (Southaven, Mississippi).

\section{Cranial Base}

The linear dimensions of the cranial base analyzed in the present study (Se-Na, Se-Ba, and Na-Ba) all showed small but significant increases. The increases in anterior cranial base length (Se-Na) and total cranial base length (Na- 
Ba) were anticipated, because previous studies have found similar changes (Sinclair and Little 1985). Increases in Sella-Nasion distance in late adolescence and adulthood are, in part, due to bony apposition of the ectocranial surface of the frontal bone. Given the ages of the subjects, most of this growth is most likely due to forward movement of Nasion, since the nasal bone is not strictly part of the cranial base, and it experiences greater growth for a longer period of time than the endochondral bones of the cranial base (Knott 1971). The observed changes in this length may also be related to the involution of the frontal sinuses with age (Rozen 2008).

In contrast, the increase in posterior cranial base length (Se-Ba) was unexpected since fusion of the spheno-occipital synchondroses typically occurs in girls in the early teens (Melsen 1969), which ought to concur with the conclusion of orthodontic treatment. There is conflicting information regarding the specific closure time of the spheno-occipital synchondroses. Studies on dry skulls (Ford 1958; Scott 1958) indicate that the closure occurs in early adulthood (17-20 years or 20-25 years). Melsen (1969) stated that the closure occurs in the interval between eruption of the second and third molars. On the other hand, a study by Ingervall and Thilander (1972) on postmortems disclosed that the closure occurs about 2 years earlier in girls than boys, and that the sphenooccipital synchondroses is never completely closed in girls older than 14 years. The average posttreatment change in the current sample was largely influenced by rather dramatic increases ( $>4 \mathrm{~mm}$ ) observed in four subjects (Figure C-2), and the amount of change observed for these four subjects may have come from continued growth at the spheno-occipital synchondroses. The results of this study suggest that the age of fusion is variable, with fusion occurring later in some individuals than in others. Another opportunity for change in the SellaBasion distance, as mentioned, is bony apposition on the dorsal aspect of the clivus, which would lengthen the Se-Ba distance. However, this seems unlikely, because it would involve bony deposition that could encroach on the spinal cord. We plotted age at the start of treatment against the amount of posttreatment growth of Sella-Basion (Figure 5-1). The slope of the least-squares line is not significant $(P=0.6211)$, which does not support the idea that younger subjects exhibit greater lengthening of the Sella-Basion dimension.

The lack of significant change in Cranial Base Angle is a finding shared with numerous other studies (Israel 1968; Roche and Lewis 1974; Lewis and Roche 1977). Like the current study, prior research found that although the anterior and posterior cranial base continued to show gradual increases with age, there was a proportional pattern of remodeling, maintaining the stability of this angle. In other words, at least after mid-adolescence, the saddle angle for a person appears fixed. 


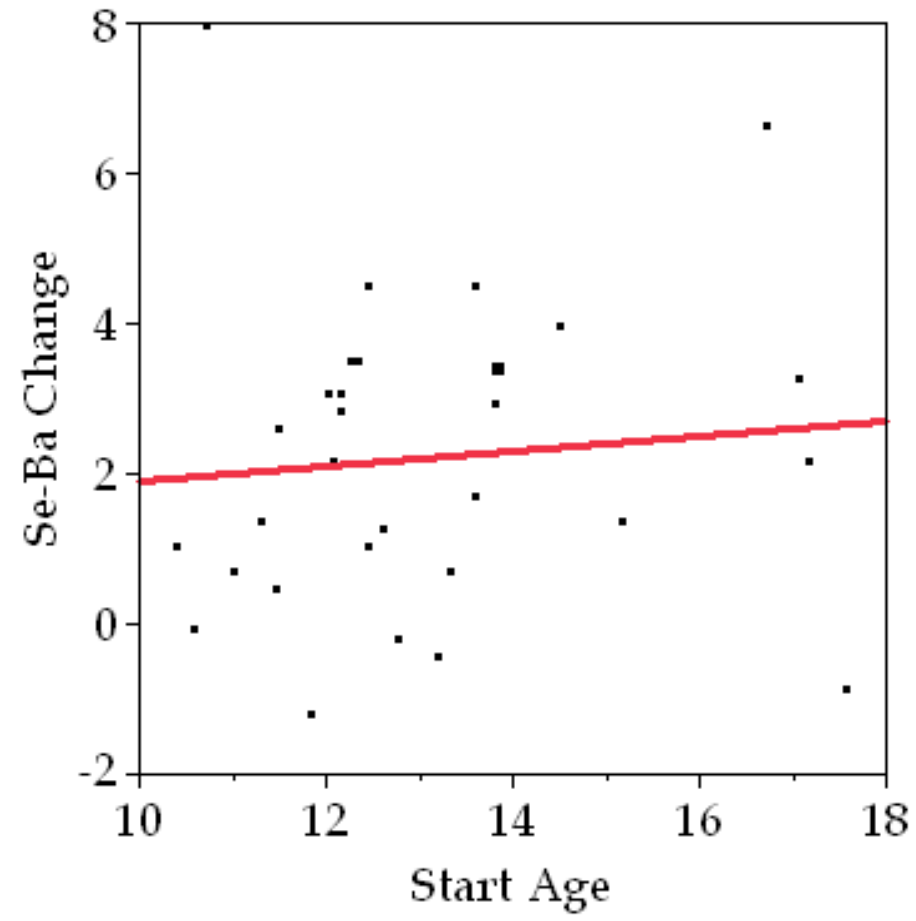

Figure 5-1. Plot of the amount of growth following treatment in the posterior cranial base against the age at the start of treatment.

There is no evidence here that fusion of the spheno-occipital synchondrosis during the mid-teens affects the amount of posttreatment growth. The slope of the least-squares line is not significant $(\mathrm{P}=0.6211)$. Adding the duration of time to the recall examination (not shown) did not improve the fit. 


\section{Midface}

The midface showed appreciable bony growth, similar to, but with greater incremental change than was seen in the cranial base. The horizontal length of the midface (Cd-A) increased, in large part, due to the forward growth of the maxilla in relation to the cranial base. This change of the midface dimension might also be caused, in part, by an increase in the nasal airway from the increased physiologic demands brought on by age (Behrents 1985). The increase in the vertical dimensions of the anterior and posterior midface (Na-ANS , SePNS), shows how that the palatal plane moved downward in both the anterior and posterior regions in conjunction with the downward and forward movement of the nasomaxillary complex as a whole.

\section{Facial Height}

The increases in anterior and posterior facial height in this sample were relatively consistent with the findings of other studies (Sinclair and Little 1985; Driscoll-Gilliland et al. 2001). The untreated females in the sample collected by Sinclair and Little experienced as much growth in posterior facial height as they did in anterior facial height $(5 \mathrm{~mm})$. This was in contrast to the current study and the previous study by Driscoll-Gilliland et al. 2001 that show that the growth of the anterior face exceeded the growth of the posterior face. The ratio of posterior facial height to anterior facial height for the subjects in this study averaged 0.67 at both the posttreatment and recall examinations. It is not surprising that this ratio remained so stable, since other studies have also found the same (Nasjleti and Kowalski 1975). On the other hand, Harris, Gardner and Vaden (1999) found a 2 percent increase in PFH/ AFH ratio in a sample of 36 extraction patients recalled at an average of 14.4 years posttreatment, most of whom were treated with Class II mechanics. They suggested that Class II correction with extractions and Class II treatment mechanics might have a favorable long-term effect on the $\mathrm{PFH} / \mathrm{AFH}$ ratio because of counterclockwise rotation of the mandible associated with a flattening (decrease) of FMA.

It is possible that treatment without extractions has less of a long-term effect on $\mathrm{PFH} / \mathrm{AFH}$ ratio than does extraction treatment, as was shown in this sample and the studies referenced above. 


\section{Maxilla}

The maxilla continued to move downward and forward in relation to the cranial base as evidenced by significant increases in the dimensions Sella-A point and Condylion-A point. This would be expected from normative growth since growth at the circum-maxillary sutures contributes to the downward and forward movement of the maxilla, and resorption of the floor of the nasal cavity and apposition at the palatal vault contributes to its downward drift (Scott 1956; Enlow and Bang 1965).

Positional change of the maxilla in relation to the anterior cranial base as measured by the Sella-Nasion-A point angle (SNA) exhibited an average increase of 0.4 degrees, which was approximately half of the change recorded for the SNB angle in this sample. This is consistent with other studies of both treated and untreated occlusions that found that the mandible typically grows about twice as much as the maxilla during the post-adolescent interval (Sinclair and Little 1985; Harris et al. 1999; Ochoa and Nanda 2004). Behrents (1985) reported that maxillary growth tapers to its adult rate at a much earlier age than does the mandible. Behrents claims that the potential for latent mandibular growth in the third decade of life is far greater than that of latent maxillary growth.

The overall stability in the angulation of the palatal plane relative to SellaNasion (mean change $=0.45^{\circ} ; \mathrm{P}=0.18$ ) is likely the result of craniofacial maturation "overpowering" the tendency of these cases to relapse from the effects of Class II treatment mechanics. Osvaldik-Trapl and Droschl (1978) found that nonextraction treatment with Class II elastics in a sample of 20 Class II adolescent patients with resulted in an average increase of 1 degree in the palatal plane to Sella-Nasion Angle at posttreatment (disclosing clockwise rotation of the palatal plane). Since most of the cases in the current sample were treated with Class II elastic forces, it is likely that these subjects would have also experienced some clockwise rotation of the palatal plane by the posttreatment examination. One might also expect the angulation of the palatal plane to relapse in a counter-clockwise direction following the conclusion of treatment. However, the actual amount of counterclockwise rotation experienced in this sample during the posttreatment interval was small and insignificant. Since this was not something that was expressed over the long-term, it is assumed that either the relapse was very small, or the normal processes of craniofacial maturation compensated for any relapse that may have occurred soon after treatment. The stability of this angle was, however, consistent with the observations of the amount of relative growth in the vertical height of the anterior midface (Na-ANS) and of the posterior midface (Se-PNS). Knowing that these two dimensions increased by nearly the same length (within $0.4 \mathrm{~mm}$ ), one would not expect much change in the angulation of the palatal plane on average. 


\section{Mandible}

There were significant changes in the mandible as evidenced by appreciable increases in Pterygoid Vertical to B point, Condylion to Gnathion, Condylion to Gonion, and Gonion to Pogonion distances. Harris, Gardner and Vaden (1999) found in a sample of 36 patients treated as adolescents that approximately $80 \%$ of the total increases in Cd-Gn, Cd-Go, and Go-Pg length during the posttreatment interval (mean $=14.4$ years) occurred during the first 5.5 years after treatment. Similarly, the high increases in mandibular length $(\mathrm{Cd}-$ $\mathrm{Gn})$, ramus length (Cd-Go), and corpus length (Go-Pg) in the present study are likely to have occurred in the first few years after treatment as the result of parapubertal growth (Hellman 1927; Nanda 1955). The increase in corpus length was likely to be, in large part, a result of growth at the gonial process, whereas the increase in ramus length is assumed to be the result of growth of the condylar process (Nanda 1955). The large range of observed changes among individuals for these three measurements (Figures C-19 through C-21) suggests that prediction of posttreatment changes in the mandible can be difficult. Regardless of the lack of predictability in growth changes that can be taken from these data, the result of the average increases in these distances was a downward and forward translation of the mandible.

The increase in chin prominence measured for this sample (NaPerp-Pg) could have been associated with the counter-clockwise rotation of the mandible, although the amount of rotation observed was only minimally significant. Enlow and Harris (1964) show that Pogonion is appositional over time, which is a more likely explanation for some of the forward displacement of Pogonion. Ricketts (1972) described the arcial growth of the mandible, suggesting that the upward and forward growth of the mandibular alveolus was, in large part, responsible for the increased protrusion of the chin with age. Moss and Salentijn (1970), similar to Ricketts, suggested that the mandible does not follow a linear pattern of growth and proposed that its growth follows a logarithmic spiral. Horizontal mandibular growth should have also contributed to the advancement of the chin relative to Nasion Perpendicular, as other studies have found a positive association between the increase in mandibular corpus length and increasing chin prominence (Stensland et al. 1988).

\section{Maxillomandibular Relationships}

Changes in the anterior-to-posterior relationships between the maxilla and mandible for this sample can be attributed to their differential growth. The observation that all 30 subjects exhibited a negative change in ANB angle (mean $=-0.35$ degrees) shows a systematically greater increase in mandibular length 
when compared to maxillary length in relation to the anterior cranial base. This finding is also supported by the differential increases seen in NaPerp-A and NaPerp-B distances (Tables A-14 and A-19), which disclose that the mandible experienced nearly twice the amount of horizontal growth than did the maxilla. The primary reason for differential growth is that the maxilla shows a rapid deceleration in growth to its adult rate at an earlier age than does the mandible (Behrents 1985; Bishara et al. 1985). According to Behrents, there is also the potential for latent mandibular growth in the third decade of life, which can be a significant contributor to differential growth.

The relative stability of the Frankfort Horizontal Plane to Mandibular Plane Angle (FMA) after treatment for this sample differs from the findings of previous studies that found that FMA typically decreases by about 1-2 degrees during the 10-year period directly following adolescence (Sinclair and Little 1985; Harris et al. 1999). Björk (1963) reported that decreases in FMA during this period are the result of the upward and forward (counterclockwise) rotation of the mandible that is secondary to remodeling at Gonion and along the mandibular border. This makes sense in analyzing the results of this study, since the observed counter-clockwise rotation of the mandible was clinically insignificant (average change in Y-Axis of -0.5 degrees), and this was likely to be associated with the small and insignificant decrease in FMA (-0.5 degrees).

The lack of post-adolescent change in skeletal facial convexity (Na-A-Pg) was anticipated based on the findings of previous longitudinal studies (Bishara and Jakobsen 1985; Harris et al. 1999). The findings of Bishara and Jakobsen show that while an increase in skeletal facial convexity can be expected for long facial types (subjects with high FMA and low PFH/AFH) between 15 and 25 years of age, either no change or decreases in skeletal facial convexity can be expected for average and short facial types. Since the average FMA $\left(20^{\circ}\right)$ and average PFH/AFH ratio (0.67) for the subjects in the present sample fit the criteria established for an average facial type by Bishara and Jakobsen, the stability in facial convexity angle for the current sample was not surprising. It is interesting, however, that the changes in facial convexity for this sample showed a high degree of inter-individual variability, in that the amount of posttreatment change ranged from -5.0 to 5.0 degrees among individuals (Figure C-27). Bishara and Jakobsen attributed the difference in changes in skeletal facial convexity among individuals to differences in the direction of mandibular growth, with longer facial types showing a greater propensity for vertical growth. The findings of Björk (1972) give support to this, since he found that "vertical growers" exhibited backwards (clockwise) rotation of the mandible and increasing facial convexity over time, while "horizontal growers" exhibited forward (counter-clockwise) mandibular rotation and decreasing facial convexity over time. 


\section{Dental Changes}

The significant uprighting of the upper and lower incisors in this sample can be attributed to a combination of maturational changes and relapse of the dentition following treatment. While maturational changes are best observed in untreated samples, changes due to relapse can only be observed in treated samples. In an untreated sample of 26 females studied by Gormely and Richardson (1999), no significant changes were found in the angulation of the upper and lower incisors from 18 years to 28 years of age. Behrents (1985) and Forsberg (1979) reported similar findings in untreated subjects observed during early adulthood, both of whom found that the maxillary incisors uprighted a small amount during this period, but overall, the U1-L1 angle showed an insignificant amount of change. The findings from a longitudinal study by Driscoll-Gilliland et al. in 2001 are of interest, since a direct comparison can be made of treated $(n=44)$ and untreated $(n=43)$ samples recalled at an average of 13 years and 9 years post-adolescence, respectively. They found that while the angulation of the upper and lower incisors showed no significant change in the untreated sample, the U1-L1 angle increased an average of 4.5 degrees in the treated sample due to uprighting of the upper and lower incisors. Similarly, Kim and Little (1999) found significant uprighting of the incisors in their treated sample, as evidenced by a decrease in IMPA by $-3.6^{\circ}$ and an increase in U1-L1 angle by $7.2^{\circ}$. These findings and the findings of the current study show that the upper and lower incisors tend to upright significantly following treatment, whereas the incisors in untreated cases show very little, if any, uprighting during the 10-year period after treatment. Thus, it is reasonable to conclude that dentofacial maturation and growth have less of an influence on incisor angulation changes than does relapse of the dentition after orthodontic treatment.

The increases in overbite and overjet observed in these cases were likely to be, in large part, a result of relapse of the dentition following treatment. Simons and Joondeph (1973) found that subjects with significant overbite and overjet at pretreatment had significantly more overbite and overjet at 10 years out of treatment than patients who did not present with much overbite and overjet at pretreatment. Those patients who had the largest decreases in overbite and overjet during treatment also had the greatest relapse at the recall examination. In contrast to what has been found in treated samples, previous studies of untreated samples during the 10-year period following adolescence found that overbite and overjet either did not change significantly (Behrents 1985; Bishara et al. 1985; Harris 1997), or overbite and overjet actually decreased (Sinclair and Little 1983). The posttreatment increases in overbite and overjet in the current sample were relatively small (mean overbite increase $=0.9 \mathrm{~mm}$; mean overjet increase $=1 \mathrm{~mm}$ ), but the findings of previous studies indicate that these changes 
were primarily the result of posttreatment relapse. The uprighting of the upper and lower incisors in the current sample is assumed to be associated the increase in overbite. Continued eruption of the incisors after treatment could have also contributed to the increase in overbite.

Mesial movement of maxillary and mandibular first molars in this sample is assumed to be the result of maturational changes in the dentition that occur with normative aging, which has been shown to occur in both treated and untreated samples from previous studies (Lundström 1969; Behrents 1985; Sinclair and Little 1985; Harris 1997; Driscoll-Gilliland et al. 2001). The mesial movement of the dentition with age is likely related to the forces of maxillary and mandibular growth. Since the growth of these two bones is primarily directed in downward and forward direction, the teeth and the periodontal ligament fibers surrounding them are also directed more mesially over time (Reitan 1969).

Much of the decrease in maxillary and mandibular arch lengths can be explained by the observed uprighting of the upper and lower incisors and by mesial movement of teeth in the buccal segments. Arch length decreases, similar to mesial molar movement, have been found in both treated and untreated samples with normal occlusions, so this was anticipated due to normal consolidation and attrition of the dentition with age (Sinclair and Little 1983; Behrents 1985; Harris 1997; Driscoll-Gilliland et al. 2001).

\section{Soft Tissue Profile Changes}

The upper and lower lips of the subjects in the current study exhibited a decrease in protrusiveness in relation to Ricketts' E-Plane by $-0.7 \mathrm{~mm}$ and -1.2 $\mathrm{mm}$, respectively. These changes are assumed to be the result of soft tissue maturational changes such as increased flaccidity of the lips and forward growth of the nose and chin, since previous studies of untreated normal occlusions have reported similar findings (Behrents 1985; Bishara et al. 1985; Zierhut et al. 2000). Bishara, Hession, and Peterson (1985) found that the distances of the upper and lower lips to Ricketts' E-plane (Pr-Pg') increased by $0.8 \mathrm{~mm}$ and $1.0 \mathrm{~mm}$, respectively, from 17 to 25 years of age. They concluded that the increases in these distances were caused by a combination of the flattening of the upper and lower lip, forward growth of the nose, and forward growth of Soft-Tissue Pogonion. All of these changes were observed in the current study, given that NaPerp-Pr distance increased by $1.9 \mathrm{~mm}$, and $\mathrm{W}$ point-Pg' distance increased by $1.5 \mathrm{~mm}$. Behrents (1985) attributed decreased lip protrusion, in part, to the uprighting of the incisors with age, which is also supported by the current study. 
The observed in increase in Merrifield's Z Angle from $76^{\circ}$ at the end of treatment to $81^{\circ}$ by the recall examination disclosed that the "most protrusive lip" and soft-tissue chin moved forward relative to the Frankfort Horizontal plane. This finding, without the consideration of other maturational changes, may seem to contradict the finding that the upper and lower lips became more retrusive relative to Ricketts' E-Plane. However, the observed increase in this angle, as previous studies have shown, is caused primarily by the increased protrusion of the chin in relation to the forehead due to continued mandibular growth rather than true lip protrusion (Bishara, Hession and Peterson 1985). Because of this, increases in $\mathrm{Z}$ Angle result in the appearance of a more "flattened" facial profile.

The Nasolabial Angle (Ls-Sn-Pr) and Mentolabial Angle (Li-B'-Pg') did not show significant change in this sample. One might expect the Nasolabial Angle to increase over time (becoming more obtuse), since the upper lip thins and becomes more flaccid with age. Previous studies of untreated subjects have shown that the Nasolabial Angle does become slightly more obtuse over the long-term, but the increases have not been shown to be not statistically significant (Behrents 1985; West and McNamara 1999; Pecora et al. 2008). West and McNamara studied the long-term cephalometric changes of 56 untreated subjects ( 29 females, 27 males) from late adolescence (mean age $=17$ years) into their late $40 \mathrm{~s}$ (mean age $=48$ years). They found that the Nasolabial Angle increased from 119 degrees to 121.5 degrees (mean increase $=2.5$ degrees) in females, while the Nasolabial Angle in males increased from 124 degrees to 124.5 degrees, which was statistically insignificant $(\mathrm{P}>0.05)$. The results of Behrents and Pecora et al. show that, although the increases in the Nasolabial Angle were clinically insignificant, there is significant thinning and elongation of the upper lip with age, and this is consistent with the changes found in the current study (Table A-55). Pecora et al. explained that a decrease in Nasolabial Angle could have occurred in some of the subjects due to structural changes in the nose, including drooping of the nasal tip and columella, but this was not usually the case.

Drooping of the upper and lower lips was an expected change of the facial soft tissues with age, as they are known to become more flaccid as muscular tone decreases (Meema et al. 1973). The increase in Sn-Ls and Sn-Li lengths by $1.7 \mathrm{~mm}$ and $2.3 \mathrm{~mm}$, respectively, in the current study were consistent with the findings of previous studies (Zierhut et al. 2000; Fudalej 2008).

Forward growth of the nose (NaPerp-Pr) and soft tissue chin (W Point$\mathrm{Pg}^{\prime}$ ) can also be attributed to normal growth. The nose grew forward an average of $1.9 \mathrm{~mm}$, and the soft-tissue chin grew forward by $1.5 \mathrm{~mm}$ on average. Both of these changes were expected, as previous studies report similar findings (Singh 
1990; Zierhut et al. 2000). The anterior location of the reference points (NaPerp and $\mathrm{W}$ point) used to measure these two soft tissue distances eliminated the possible influences of maxillary and mandibular growth occurring posterior to these points. An exception to this is the influence of appositional changes at Pogonion on the $\mathrm{W}$ Point-Pg' distance, which resulted in additional forward movement of the overlying soft tissue chin. 


\section{CHAPTER 6. SUMMARY AND CONCLUSIONS}

This longitudinal cephalometric study examined 30 females treated during adolescence by a single, experienced orthodontist and then re-examined them at an average of 28 years of age (mean recall $=12.5$ years out of treatment). As such, the current study examined the changes that occurred from a period (on average) from late adolescence into early adulthood. All of the subjects in this sample presented with a Class II division I malocclusion at pretreatment, and all were treated orthodontically without extractions. Conclusions drawn from this study were:

- The cranial base exhibited significant lengthening in 3 dimensions (Se-Na, Se-Ba, and Na-Ba increased), mostly due to late adolescent growth. The continued increase in Se-Ba distance after adolescence in this sample suggested that the age of fusion of the spheno-occipital synchondroses was variable, with fusion occurring later in some individuals than in others.

- The vertical dimensions of the anterior and posterior face increased significantly, primarily due to growth-related change. The ratio of $\mathrm{PFH} / \mathrm{AFH}$ of 0.67 remained stable over the long-term, disclosing a proportionally balanced amount of growth between the anterior and posterior face.

- Overall, maxillary growth was in a down and forward direction, with total down and forward directional growth (Se-A) totaling $4.3 \mathrm{~mm}$.

- Overall, mandibular growth was in a down and forward direction, with total down and forward directional growth (Cd-Gn) totaling $6.6 \mathrm{~mm}$.

- Late mandibular growth after adolescence exceeds late growth in the maxilla by nearly twice as much

- The mandible continues to lengthen vertically (Cd-Go), horizontally (Go$\mathrm{Pg})$, and diagonally (Cd-Gn), but the large range of observed changes makes predictability of change in the mandible difficult. Most of this change is growth-related, but apposition at Gonion and Pogonion are believed to contribute to the overall change.

- The upper and lower incisors are likely to upright by some degree after non-extraction treatment due to relapse of the dentition in the first few years after treatment. While a small of uprighting has been shown for the upper incisors in previous studies of untreated subjects, larger changes in both dental arches have been isolated to treated samples.

- Overbite and overjet relapse to some degree after treatment, and this should be expected, since many Class II patients possess excessive initial overbite and overjet. 
- Maxillary and mandibular arch lengths decrease after treatment. Studies of untreated subjects show that this is a normal maturational change associated with aging due to consolidation of the dentition. Contributing factors for the decreases observed in the current sample were the mesial movement of the first molars and the uprighting of the upper and lower incisors.

- Soft tissue profiles become progressively more flattened after treatment. This was disclosed in the current sample by the increase in $\mathrm{Z}$ Angle and the increased retrusion of the upper and lower lips relative to the E Plane. This flattening of the facial profile is attributable to several factors:

1. The lips become more flaccid and less prominent with normal aging.

2. The nose and soft tissue chin continue to grow forward.

3. The upper and lower lips droop inferiorly with age.

The present study has shown that significant posttreatment change can be expected during a 12-year period following orthodontic treatment from late adolescence into early adulthood. Craniofacial growth, maturational changes associated with normal aging, and posttreatment relapse of the dentition are changes that occur simultaneously to produce the overall posttreatment change. 


\section{LIST OF REFERENCES}

Ahn JG, Schneider BJ. Cephalometric appraisal of posttreatment vertical changes in adult orthodontic patients. Am J Orthod Dentofacial Orthop 2000;118:378-84.

Akgül AA, Toygar TU. Natural craniofacial changes in the third decade of life: a longitudinal study. Am J Orthod Dentofacial Orthop 2002;122:512-22.

Al Yami EA, Kujipers-Jagtman AM, Van't Hof MA. Stability of orthodontic treatment outcome: follow-up until 10 years postretention. Am J Orthod Dentofacial Orthop 1999;115:300-4.

Bärbel KN, Fishbach H, Schwarze CW. Treatment and postretention changes in dental arch width dimensions-a long-term evaluation of influencing cofactors. Am J Orthod Dentofacial Orthop 1996;109:368-78.

Bishara SE, Chadha JM, Potter RB. Stability of intercanine width, overbite, and overjet correction. Am J Orthod 1973;63:588-94.

Bishara SE, Hession TJ, Peterson LC. Longitudinal soft-tissue profile changes: a study of three analyses. Am J Orthod Dentofacial Orthop 1985;88:209-23.

Bishara SE, Jakobsen JR. Longitudinal changes in three normal facial types. Am J Orthod Dentofacial Orthop 1985;88:466-502.

Behrents RG. Growth in the aging craniofacial skeleton. Monograph No. 17, Craniofacial Growth Series. Ann Arbor: 1985. University of Michigan.

Behrents RG. An atlas of growth in the aging craniofacial skeleton. Monograph No. 18, Craniofacial Growth Series. Ann Arbor: 1986. University of Michigan.

Blake M, Bibby K. Retention and stability: a review of the literature. Am J Orthod Dentofacial Orthop 1998;114:299-306.

Björk A, Skieller V. Facial development and tooth eruption: an implant an implant study at the age of puberty. Am J Orthod 1972;62:339-83.

Bresonis WL, Grewe JM. Treatment and posttreatment changes in orthodontic cases: overbite and overjet. Angle Orthod 1974;44:295-9.

Burke SP, Silveira AM, Goldsmith LJ, Yancey JM, Van Stewart A, Scarfe WC. A meta-analysis of mandibular intercanine width in treatment and postretention. Angle Orthod 1998;68:53-60. 
Buschang PH, Shulman JD. Incisor crowding in untreated persons 15-50 years of age: United States, 1988-1994. Angle Orthod 2003;73:502-8.

Chung C, Mongiovi VD. Craniofacial growth in untreated skeletal Class I subjects with low, average, and high MP-SN angles: a longitudinal study. Am J Orthod Dentofacial Orthop 2003;124:670-8.

Cohen JM. Comparing digital and conventional cephalometric radiographs. Am J Orthod Dentofacial Orthop 2005;128:157-60.

Cook DR, Harris EF, Vaden JL. Comparison of university and private-practice orthodontic treatment outcomes with the American Board of Orthodontics objective grading system. Am J Orthod Dentofacial Orthop 2005;127:70712.

Dahlberg G. Statistical methods for medical and biological students. London: George Allen and Unwin, Ltd, 1940.

De La Cruz A, Sampson P, Little RM, Årtun J, Shapiro PA. Long-term changes in arch form after orthodontic treatment and retention. Am J Orthod Dentofacial Orthop 1995;107:518-30.

Driscoll-Gilliland J, Buschang MA, Behrents RG. An evaluation of growth and stability in untreated and treated subjects. Am J Orthod Dentofacial Orthop 2001;120:588-97

Elms TN, Buschang PH, Alexander RG. Long-term stability of Class II, Division 1 nonextraction cervical facebow therapy: I. Model analysis. Am J Orthod Dentofacial Orthop 1996;109:271-6.

Elms TN, Buschang PH, Alexander RG. Long-term stability of Class II, Division 1 nonextraction cervical facebow therapy: II. Cephalometric analysis. Am J Orthod Dentofacial Orthop 1996;109:386-92.

Enlow DH, Bang S. Growth and remodeling of the human maxilla. Am J Orthod 1965;51:446-64.

Eslambolchi S, Woodside DG, Rossouw PE. A descriptive study of mandibular incisor alignment in untreated subjects. Am J Orthod Dentofacial Orthop 2008;133:343-53.

Finch CE, Schneider EL. Handbook of the biology of aging, $2^{\text {nd }}$ ed. New York: Van Nostrand Rheinhold, 1985.

Forsberg CM. Facial morphology and aging: a longitudinal cephalometric investigation of young adults. Eur J Orthod 1979;1:15-23.

Ford EHR. Growth of human cranial base. Am J Orthod 1958;44:498-506. 
Fudalej P. Long-term changes of upper lip position relative to the incisal edge. Am J Orthod Dentofacial Orthop 2008;133:204-9.

Gormely JS, Richardson ME. Linear and angular changes in dento-facial dimensions in the third decade. Br J Orthod 1999;26:51-5.

Harris EF, Behrents R. The intrinsic stability of Class I molar relationship: a longitudinal study of untreated cases. Am J Orthod Dentofacial Orthop 1988;94:63-7.

Harris EF. A longitudinal study of arch size and arch form in untreated adults. Am J Orthod Dentofacial Orthop 1997;111:419-27.

Harris EF, Gardner RZ, Vaden JL. A longitudinal cephalometric study of postorthodontic craniofacial changes. Am J Orthod Dentofacial Orthop 1999;115:77-82.

Hellman M. Changes in the human face brought on by development. Int J Orthod 1927;13:475-516.

Houston WJ. The analysis of errors in orthodontic measurements. Am J Orthod 1983;83:382-90.

Ingervall B, Thilander B. The human spheno-occipital synchondrosis: I, time of closure appraised macroscopically. Acta Odontol Scand 1972;30:349-56.

Israel $\mathrm{H}$. Continuing growth in the human cranial skeleton. Arch Oral Biol 1968;13:133-8.

Knott VB. Change in cranial base measures of human males and females from age 6 years to early adulthood. Growth 1971;35:145-58.

Lewis AB, Roche AF. The saddle angle: constancy or change? AngleOrthod 1977;47:46-54.

Little RM, Riedel RA, Årtun J. An evaluation of changes in mandibular anterior alignment from 10 to 20 years postretention. Am J Orthod Dentofacial Orthop 1988;93:423-8.

Little RM, Riedel RA, Stein A. Mandibular arch length increase during the mixed dentition: postretention evaluation of stability and relapse. Am J Orthod Dentofacial Orthop 1990;97:393-404.

Little RM, Wallen T, Riedel RA. Stability and relapse of mandibular anterior alignment: first premolar extraction cases treated by traditional edgewise orthodontics. Am J Orthod Dentofacial Orthop 1981;80:349-65. 
Little RM. Stability and relapse: early treatment of arch length deficiency. Am J Orthod Dentofacial Orthop 2002;121:578-81.

Meema S, Reed DB, Meema HE. Age trends of bone mineral mass, muscle width, and subcutaneous fat in normals and osteoporotics. Calcif Tissue Int 1973;12:101-12.

Melsen B. Time of closure of the sphenooccipital synchondrosis determined on dry skulls: a radiographic craniometric study. Acta Odontol Scand 1969;27:73-90.

Moss ML, Salentijn L. Logarithmic growth of the human mandible. Acta Anat 1970;77:341-60.

Nance H. The limitations of orthodontic treatment. Am J Orthod Oral Surg 1947;33:253-301.

Nanda R. The rates of growth of several facial components measured from serial cephalometric roentograms. Am J Orthod 1955;41:658-73.

Nasjleti CE, Kowalski CJ. Stability of upper face height-total face height ratio with increasing age. J Dent Res 1975;54:1241.

Ochoa BK, Nanda RS. Comparison of maxillary and mandibular growth. Am J Orthod Dentofacial Orthop 2004;125:148-59.

Oppenheim A. The crisis in orthodontia. Int J Orthod Dent Child 1934; 20:64044.

Ormiston JP, Huang GJ, Little RM, Decker JD, Seuk GD. Retrospective analysis of long-term stable and unstable orthodontic treatment outcomes. Am J Orthod Dentofacial Orthop 2005;128:568-74.

Pecora NG, Bacetti T, McNamara JA. The aging craniofacial complex: a longitudinal cephalometric study from late adolescence to late adulthood. Am J Orthod Dentofacial Orthop 2008;134:496-505.

Pepicelli A, Woods M, Briggs C. The mandibular muscles and their importance in orthodontics: a contemporary review. Am J Orthod Dentofacial Orthop $2005 ; 128: 774-80$.

Proffit WR. Equilibrium theory revisited: factors influencing position of the teeth. Angle Orthod 1978;48:175-86.

Reitan K. Principles of retention and avoidance of posttreatment relapse. Am J Orthod 1969;55:776-90. 
Ricketts RM. Planning treatment on the basis of the facial pattern and an estimate of its growth. Angle Orthod 1957;27:14-37.

Ricketts RM. A principal of arcial growth of the mandible. Angle Orthod 1972;42:368-86.

Rothe LE, Bollen AM, Little RM, Herring SW, Chaison JB, Chen CS, Hollender LG. Trabecular and cortical bone as risk factors for orthodontic relapse. Am J Orthod Dentofacial Orthop 2006;129:476-84.

Rozen WM, Joseph S, Lo PA. Spontaneous involution of two sinus pericranii: a unique case and review of the literature. J Clin Neurosci 2008;15:833-5.

Sadowsky C, Schneider BJ, BeGole EA, Tahir E. Long-term stability after orthodontic treatment: non-extraction with prolonged retention. Am J Orthod Dentofacial Orthop 1994;106:243-9.

Scott JH. Growth at facial sutures. Am J Orthod 1956;42:381-87.

Scott JH. The growth of human skull. J Dent Assoc S Afr 1958;16:319.

Sokal RR, Rohlf FJ. Biometry: the principles and practice of statistics in biological research, 3rd ed. San Francisco: WH Freeman and Company, 1995.

Shields TE, Little RM, Chapko MK. Stability and relapse of mandibular anterior alignment: a cephalometric appraisal of first premolar extraction cases treated by traditional edgewise orthodontics. Am J Orthod Dentofacial Orthop 1985;87:27-38.

Sinclair PM, Little RM. Dentofacial maturation of untreated normals. Am J Orthod Dentofacial Orthop 1985;88:146-56.

Sinclair PM, Little RM. Maturation of untreated normal occlusions. Am J Orthod Dentofacial Orthop 1983;83:114-23.

Singh RN. Changes in the soft tissue chin after orthodontic treatment. Am J Orthod Dentofacial Orthop 1990;98:41-6.

Stensland A, Wisth PJ, Boe OE. Dentofacial changes in children with negative overjet treated by a combined orthodontic and orthopedic approach. Eur J Orthod 1988;10:39-51.

Tukey JW. Exploratory data analysis. Reading, Mass: Addision-Wesley, 1977.

Udhe MD, Sadowsky C, BeGole EA. Long term stability of dental relationships after orthodontic treatment. Angle Orthod 1983;53:240-52. 
Weinberger BW. Orthodontics, an historical review of its origin and evolution. Vol. 1. St. Louis: CV Mosby; 1926.

West KS, McNamara JA. Changes in the craniofacial complex from adolescence to midadulthood: a cephalometric study. Am J Orthod Dentofacial Orthop 1999;115:175-82.

Zierhut EC, Joondeph DR, Ártun J, Little RM. Long-term profile changes associated with successfully treated extraction and nonextraction Class II division 1 malocclusions. Angle Orthod 2000;70:208-19. 
APPENDIX A.

DESCRIPTIVE STATISTICS AND TESTS FOR POSTTREATMENT CHANGES 
Table A-1. Descriptive statistics and tests for significant posttreatment changes based on repeated measurements for Sella-Nasion (mm).

\begin{tabular}{|c|c|c|c|c|c|c|}
\hline \multirow[b]{2}{*}{ Variable } & \multicolumn{2}{|c|}{ End of Treatment } & \multicolumn{2}{|c|}{ Recall Examination } & \multicolumn{2}{|c|}{ Posttreatment Change } \\
\hline & $\mathrm{T} 1$ & $\mathrm{~T} 2$ & $\mathrm{~T} 1$ & $\mathrm{~T} 2$ & $\mathrm{~T} 1$ & $\mathrm{~T} 2$ \\
\hline Mean & 71.90 & 71.88 & 74.63 & 74.62 & 2.72 & 2.74 \\
\hline Standard deviation & 2.46 & 2.44 & 2.37 & 2.36 & 0.46 & 0.48 \\
\hline Standard error & 0.45 & 0.45 & 0.43 & 0.43 & 0.08 & 0.09 \\
\hline Upper $95 \%$ of mean & 72.82 & 72.80 & 75.51 & 75.51 & 2.89 & 2.92 \\
\hline Lower $95 \%$ of mean & 70.98 & 70.97 & 73.74 & 73.74 & 2.55 & 2.56 \\
\hline Sample size & 30 & 30 & 30 & 30 & 30 & 30 \\
\hline One-sample t-test & & & & & 32.74 & 31.25 \\
\hline P-value & & & & & $<0.0001$ & $<0.0001$ \\
\hline
\end{tabular}

Table A-2. Descriptive statistics and tests for significant posttreatment changes based on repeated measurements for Sella-Basion ( $\mathrm{mm})$.

\begin{tabular}{|c|c|c|c|c|c|c|}
\hline \multirow[b]{2}{*}{ Variable } & \multicolumn{2}{|c|}{ End of Treatment } & \multicolumn{2}{|c|}{$\underline{\text { Recall Examination }}$} & \multicolumn{2}{|c|}{$\underline{\text { Posttreatment Change }}$} \\
\hline & $\mathrm{T} 1$ & $\mathrm{~T} 2$ & $\mathrm{~T} 1$ & $\overline{\mathrm{T} 2}$ & $\mathrm{~T} 1$ & $\mathrm{~T} 2$ \\
\hline Mean & 41.43 & 41.40 & 43.64 & 43.63 & 2.20 & 2.23 \\
\hline Standard deviation & 2.81 & 2.84 & 2.71 & 2.72 & 2.09 & 2.11 \\
\hline Standard error & 0.51 & 0.52 & 0.50 & 0.50 & 0.38 & 0.39 \\
\hline Upper $95 \%$ of mean & 42.48 & 42.47 & 44.65 & 44.65 & 2.98 & 3.02 \\
\hline Lower $95 \%$ of mean & 40.38 & 40.34 & 42.62 & 42.62 & 1.42 & 1.44 \\
\hline Sample size & 30 & 30 & 30 & 30 & 30 & 30 \\
\hline One-sample t-test & & & & & 5.77 & 5.79 \\
\hline P-value & & & & & $<0.0001$ & $<0.0001$ \\
\hline
\end{tabular}


Table A-3. Descriptive statistics and tests for significant posttreatment changes based on repeated measurements for Nasion-Basion (mm).

\begin{tabular}{|c|c|c|c|c|c|c|}
\hline \multirow[b]{2}{*}{ Variable } & \multicolumn{2}{|c|}{ End of Treatment } & \multicolumn{2}{|c|}{$\underline{\text { Recall Examination }}$} & \multicolumn{2}{|c|}{ Posttreatment Change } \\
\hline & $\mathrm{T} 1$ & $\mathrm{~T} 2$ & $\mathrm{~T} 1$ & $\mathrm{~T} 2$ & $\mathrm{~T} 1$ & $\mathrm{~T} 2$ \\
\hline Mean & 104.23 & 104.21 & 109.74 & 109.73 & 5.51 & 5.52 \\
\hline Standard deviation & 4.25 & 4.24 & 3.71 & 3.64 & 1.71 & 1.70 \\
\hline Standard error & 0.78 & 0.77 & 0.68 & 0.66 & 0.31 & 0.31 \\
\hline Upper $95 \%$ of mean & 105.82 & 105.80 & 111.13 & 111.09 & 6.15 & 6.15 \\
\hline Lower $95 \%$ of mean & 102.64 & 102.63 & 108.36 & 108.37 & 4.88 & 4.88 \\
\hline Sample size & 30 & 30 & 30 & 30 & 30 & 30 \\
\hline One-sample t-test & & & & & 17.69 & 17.79 \\
\hline P-value & & & & & $<0.0001$ & $<0.0001$ \\
\hline
\end{tabular}

Table A-4. Descriptive statistics and tests for significant posttreatment changes based on repeated measurements for Nasion-Sella-Basion Angle $\left(^{\circ}\right)$.

\begin{tabular}{|c|c|c|c|c|c|c|}
\hline \multirow[b]{2}{*}{ Variable } & \multicolumn{2}{|c|}{ End of Treatment } & \multicolumn{2}{|c|}{ Recall Examination } & \multicolumn{2}{|c|}{ Posttreatment Change } \\
\hline & $\mathrm{T} 1$ & $\mathrm{~T} 2$ & $\mathrm{~T} 1$ & $\mathrm{~T} 2$ & $\mathrm{~T} 1$ & $\mathrm{~T} 2$ \\
\hline Mean & 133.52 & 133.55 & 133.54 & 133.48 & 0.02 & -0.07 \\
\hline Standard deviation & 3.38 & 3.40 & 3.73 & 3.74 & 2.04 & 2.12 \\
\hline Standard error & 0.62 & 0.62 & 0.68 & 0.68 & 0.37 & 0.39 \\
\hline Upper $95 \%$ of mean & 134.78 & 134.81 & 134.94 & 134.88 & 0.78 & 0.73 \\
\hline Lower $95 \%$ of mean & 132.26 & 132.28 & 132.15 & 132.08 & -0.74 & -0.86 \\
\hline Sample size & 30 & 30 & 30 & 30 & 30 & 30 \\
\hline One-sample t-test & & & & & 0.05 & -0.17 \\
\hline P-value & & & & & 0.9575 & 0.8646 \\
\hline
\end{tabular}


Table A-5. Descriptive statistics and tests for significant posttreatment changes based on repeated measurements for Nasion-Anterior Nasal Spine ( $\mathrm{mm})$.

\begin{tabular}{|c|c|c|c|c|c|c|}
\hline \multirow[b]{2}{*}{ Variable } & \multicolumn{2}{|c|}{ End of Treatment } & \multicolumn{2}{|c|}{ Recall Examination } & \multicolumn{2}{|c|}{ Posttreatment Change } \\
\hline & $\mathrm{T} 1$ & $\mathrm{~T} 2$ & $\mathrm{~T} 1$ & $\mathrm{~T} 2$ & $\mathrm{~T} 1$ & $\mathrm{~T} 2$ \\
\hline Mean & 49.88 & 49.89 & 53.01 & 53.01 & 3.13 & 3.12 \\
\hline Standard deviation & 2.60 & 2.60 & 2.53 & 2.53 & 1.27 & 1.28 \\
\hline Standard error & 0.48 & 0.47 & 0.46 & 0.46 & 0.23 & 0.23 \\
\hline Upper $95 \%$ of mean & 50.85 & 50.86 & 53.95 & 53.95 & 3.60 & 3.60 \\
\hline Lower $95 \%$ of mean & 48.91 & 48.92 & 52.06 & 52.06 & 2.65 & 2.64 \\
\hline Sample size & 30 & 30 & 30 & 30 & 30 & 30 \\
\hline One-sample t-test & & & & & 13.49 & 13.37 \\
\hline P-value & & & & & $<0.0001$ & $<0.0001$ \\
\hline
\end{tabular}

Table A-6. Descriptive statistics and tests for significant posttreatment changes based on repeated measurements for Sella-Posterior Nasal Spine (mm).

\begin{tabular}{|c|c|c|c|c|c|c|}
\hline \multirow[b]{2}{*}{ Variable } & \multicolumn{2}{|c|}{ End of Treatment } & \multicolumn{2}{|c|}{ Recall Examination } & \multicolumn{2}{|c|}{ Posttreatment Change } \\
\hline & $\mathrm{T} 1$ & $\mathrm{~T} 2$ & $\mathrm{~T} 1$ & $\mathrm{~T} 2$ & $\mathrm{~T} 1$ & $\mathrm{~T} 2$ \\
\hline Mean & 52.88 & 52.90 & 55.62 & 55.60 & 2.73 & 2.69 \\
\hline Standard deviation & 2.54 & 2.56 & 2.92 & 2.92 & 2.11 & 2.14 \\
\hline Standard error & 0.46 & 0.47 & 0.53 & 0.53 & 0.38 & 0.39 \\
\hline Upper $95 \%$ of mean & 53.83 & 53.86 & 56.70 & 56.69 & 3.52 & 3.49 \\
\hline Lower $95 \%$ of mean & 51.94 & 51.95 & 54.53 & 54.51 & 1.95 & 1.89 \\
\hline Sample size & 30 & 30 & 30 & 30 & 30 & 30 \\
\hline One-sample t-test & & & & & 7.10 & 6.90 \\
\hline P-value & & & & & $<0.0001$ & $<0.0001$ \\
\hline
\end{tabular}


Table A-7. Descriptive statistics and tests for significant posttreatment changes based on repeated measurements for Condylion-A point (mm).

\begin{tabular}{|c|c|c|c|c|c|c|}
\hline \multirow[b]{2}{*}{ Variable } & \multicolumn{2}{|c|}{ End of Treatment } & \multicolumn{2}{|c|}{$\underline{\text { Recall Examination }}$} & \multicolumn{2}{|c|}{ Posttreatment Change } \\
\hline & $\mathrm{T} 1$ & $\mathrm{~T} 2$ & $\mathrm{~T} 1$ & $\mathrm{~T} 2$ & $\mathrm{~T} 1$ & $\mathrm{~T} 2$ \\
\hline Mean & 88.99 & 88.99 & 92.54 & 92.52 & 3.55 & 3.52 \\
\hline Standard deviation & 4.87 & 4.85 & 4.64 & 4.63 & 1.91 & 1.92 \\
\hline Standard error & 0.89 & 0.89 & 0.85 & 0.85 & 0.35 & 0.35 \\
\hline Upper $95 \%$ of mean & 90.80 & 90.80 & 94.27 & 94.25 & 4.26 & 4.24 \\
\hline Lower $95 \%$ of mean & 87.17 & 87.18 & 90.80 & 90.79 & 2.84 & 2.80 \\
\hline Sample size & 30 & 30 & 30 & 30 & 30 & 30 \\
\hline One-sample t-test & & & & & 10.20 & 10.04 \\
\hline P-value & & & & & $<0.0001$ & $<0.0001$ \\
\hline
\end{tabular}

Table A-8. Descriptive statistics and tests for significant posttreatment changes based on repeated measurements for Nasion-Palatal Plane (mm).

\begin{tabular}{|c|c|c|c|c|c|c|}
\hline \multirow[b]{2}{*}{ Variable } & \multicolumn{2}{|c|}{ End of Treatment } & \multicolumn{2}{|c|}{ Recall Examination } & \multicolumn{2}{|c|}{ Posttreatment Change } \\
\hline & $\mathrm{T} 1$ & $\mathrm{~T} 2$ & $\mathrm{~T} 1$ & $\mathrm{~T} 2$ & $\mathrm{~T} 1$ & $\mathrm{~T} 2$ \\
\hline Mean & 49.88 & 49.86 & 53.01 & 53.02 & 3.13 & 3.17 \\
\hline Standard deviation & 2.60 & 2.61 & 2.53 & 2.56 & 1.27 & 1.28 \\
\hline Standard error & 0.48 & 0.48 & 0.46 & 0.47 & 0.23 & 0.23 \\
\hline Upper $95 \%$ of mean & 50.85 & 50.83 & 53.95 & 53.98 & 3.60 & 3.65 \\
\hline Lower $95 \%$ of mean & 48.91 & 48.88 & 52.06 & 52.07 & 2.65 & 2.69 \\
\hline Sample size & 30 & 30 & 30 & 30 & 30 & 30 \\
\hline One-sample t-test & & & & & 13.49 & 13.58 \\
\hline P-value & & & & & $<0.0001$ & $<0.0001$ \\
\hline
\end{tabular}


Table A-9. Descriptive statistics and tests for significant posttreatment changes based on repeated measurements for Menton-Palatal Plane ( $\mathrm{mm})$.

\begin{tabular}{|c|c|c|c|c|c|c|}
\hline \multirow[b]{2}{*}{ Variable } & \multicolumn{2}{|c|}{ End of Treatment } & \multicolumn{2}{|c|}{$\underline{\text { Recall Examination }}$} & \multicolumn{2}{|c|}{ Posttreatment Change } \\
\hline & $\mathrm{T} 1$ & $\mathrm{~T} 2$ & $\mathrm{~T} 1$ & $\mathrm{~T} 2$ & $\mathrm{~T} 1$ & $\mathrm{~T} 2$ \\
\hline Mean & 64.39 & 64.36 & 68.73 & 68.73 & 4.34 & 4.37 \\
\hline Standard deviation & 5.79 & 5.78 & 6.45 & 6.44 & 2.00 & 1.97 \\
\hline Standard error & 1.06 & 1.05 & 1.18 & 1.18 & 0.36 & 0.36 \\
\hline Upper $95 \%$ of mean & 66.55 & 66.52 & 71.14 & 71.14 & 5.09 & 5.11 \\
\hline Lower $95 \%$ of mean & 62.23 & 62.20 & 66.32 & 66.33 & 3.59 & 3.64 \\
\hline Sample size & 30 & 30 & 30 & 30 & 30 & 30 \\
\hline One-sample t-test & & & & & 11.90 & 12.18 \\
\hline P-value & & & & & $<0.0001$ & $<0.0001$ \\
\hline
\end{tabular}

Table A-10. Descriptive statistics and tests for significant posttreatment changes based on repeated measurements for Nasion-Menton (mm).

\begin{tabular}{|c|c|c|c|c|c|c|}
\hline \multirow[b]{2}{*}{ Variable } & \multicolumn{2}{|c|}{ End of Treatment } & \multicolumn{2}{|c|}{ Recall Examination } & \multicolumn{2}{|c|}{ Posttreatment Change } \\
\hline & $\mathrm{T} 1$ & $\mathrm{~T} 2$ & $\mathrm{~T} 1$ & $\mathrm{~T} 2$ & $\mathrm{~T} 1$ & $\mathrm{~T} 2$ \\
\hline Mean & 112.86 & 112.88 & 120.05 & 120.04 & 7.19 & 7.16 \\
\hline Standard deviation & 6.87 & 6.87 & 7.05 & 7.07 & 2.08 & 2.11 \\
\hline Standard error & 1.25 & 1.25 & 1.29 & 1.29 & 0.38 & 0.39 \\
\hline Upper $95 \%$ of mean & 115.43 & 115.45 & 122.69 & 122.68 & 7.97 & 7.94 \\
\hline Lower $95 \%$ of mean & 110.30 & 110.32 & 117.42 & 117.40 & 6.41 & 6.37 \\
\hline Sample size & 30 & 30 & 30 & 30 & 30 & 30 \\
\hline One-sample t-test & & & & & 18.94 & 18.57 \\
\hline P-value & & & & & $<0.0001$ & $<0.0001$ \\
\hline
\end{tabular}


Table A-11. Descriptive statistics and tests for significant posttreatment changes based on repeated measurements for Sella-Gonion ( $\mathrm{mm})$.

\begin{tabular}{|c|c|c|c|c|c|c|}
\hline \multirow[b]{2}{*}{ Variable } & \multicolumn{2}{|c|}{ End of Treatment } & \multicolumn{2}{|c|}{$\underline{\text { Recall Examination }}$} & \multicolumn{2}{|c|}{ Posttreatment Change } \\
\hline & $\mathrm{T} 1$ & $\mathrm{~T} 2$ & $\mathrm{~T} 1$ & $\mathrm{~T} 2$ & $\mathrm{~T} 1$ & $\mathrm{~T} 2$ \\
\hline Mean & 75.64 & 75.64 & 79.79 & 79.76 & 4.15 & 4.12 \\
\hline Standard deviation & 4.04 & 4.05 & 4.87 & 4.85 & 3.58 & 3.59 \\
\hline Standard error & 0.74 & 0.74 & 0.89 & 0.89 & 0.65 & 0.66 \\
\hline Upper $95 \%$ of mean & 77.15 & 77.15 & 81.60 & 81.58 & 5.48 & 5.46 \\
\hline Lower $95 \%$ of mean & 74.13 & 74.13 & 77.97 & 77.95 & 2.81 & 2.78 \\
\hline Sample size & 30 & 30 & 30 & 30 & 30 & 30 \\
\hline One-sample t-test & & & & & 6.34 & 6.29 \\
\hline P-value & & & & & $<0.0001$ & $<0.0001$ \\
\hline
\end{tabular}

Table A-12. Descriptive statistics and tests for significant posttreatment changes based on repeated measurements for Posterior Facial Height/ Anterior Facial Height Ratio.

\begin{tabular}{|c|c|c|c|c|c|c|}
\hline \multirow[b]{2}{*}{ Variable } & \multicolumn{2}{|c|}{ End of Treatment } & \multicolumn{2}{|c|}{$\underline{\text { Recall Examination }}$} & \multicolumn{2}{|c|}{$\underline{\text { Posttreatment Change }}$} \\
\hline & $\mathrm{T} 1$ & $\mathrm{~T} 2$ & $\mathrm{~T} 1$ & $\mathrm{~T} 2$ & $\mathrm{~T} 1$ & $\mathrm{~T} 2$ \\
\hline Mean & 0.67 & 0.67 & 0.67 & 0.67 & 0.00 & 0.00 \\
\hline Standard deviation & 0.05 & 0.05 & 0.06 & 0.06 & 0.03 & 0.03 \\
\hline Standard error & 0.01 & 0.01 & 0.01 & 0.01 & 0.01 & 0.01 \\
\hline Upper $95 \%$ of mean & 0.69 & 0.69 & 0.69 & 0.69 & 0.01 & 0.01 \\
\hline Lower $95 \%$ of mean & 0.66 & 0.65 & 0.65 & 0.64 & -0.02 & -0.02 \\
\hline Sample size & 30 & 30 & 30 & 30 & 30 & 30 \\
\hline One-sample t-test & & & & & -0.90 & -0.82 \\
\hline P-value & & & & & 0.3771 & 0.4212 \\
\hline
\end{tabular}


Table A-13. Descriptive statistics and tests for significant posttreatment changes based on repeated measurements for Sella-Nasion-A Point Angle $\left(^{\circ}\right)$.

\begin{tabular}{|c|c|c|c|c|c|c|}
\hline \multirow[b]{2}{*}{ Variable } & \multicolumn{2}{|c|}{ End of Treatment } & \multicolumn{2}{|c|}{ Recall Examination } & \multicolumn{2}{|c|}{ Posttreatment Change } \\
\hline & $\mathrm{T} 1$ & $\mathrm{~T} 2$ & $\mathrm{~T} 1$ & $\mathrm{~T} 2$ & $\mathrm{~T} 1$ & $\mathrm{~T} 2$ \\
\hline Mean & 79.92 & 79.86 & 80.36 & 80.36 & 0.44 & 0.51 \\
\hline Standard deviation & 3.71 & 3.68 & 3.73 & 3.73 & 0.17 & 0.41 \\
\hline Standard error & 0.68 & 0.67 & 0.68 & 0.68 & 0.03 & 0.08 \\
\hline Upper $95 \%$ of mean & 81.31 & 81.23 & 81.76 & 81.75 & 0.50 & 0.66 \\
\hline Lower $95 \%$ of mean & 78.54 & 78.48 & 78.97 & 78.97 & 0.38 & 0.35 \\
\hline Sample size & 30 & 30 & 30 & 30 & 30 & 30 \\
\hline One-sample t-test & & & & & 13.90 & 6.70 \\
\hline P-value & & & & & $<0.0001$ & $<0.0001$ \\
\hline
\end{tabular}

Table A-14. Descriptive statistics and tests for significant posttreatment changes based on repeated measurements for Nasion Perpendicular-A point (mm).

\begin{tabular}{|c|c|c|c|c|c|c|}
\hline \multirow[b]{2}{*}{ Variable } & \multicolumn{2}{|c|}{ End of Treatment } & \multicolumn{2}{|c|}{ Recall Examination } & \multicolumn{2}{|c|}{ Posttreatment Change } \\
\hline & $\mathrm{T} 1$ & $\mathrm{~T} 2$ & $\mathrm{~T} 1$ & $\mathrm{~T} 2$ & $\mathrm{~T} 1$ & $\mathrm{~T} 2$ \\
\hline Mean & -0.54 & -0.56 & 0.87 & 0.88 & 1.41 & 1.43 \\
\hline Standard deviation & 2.29 & 2.29 & 2.31 & 2.30 & 1.16 & 1.15 \\
\hline Standard error & 0.42 & 0.42 & 0.42 & 0.42 & 0.21 & 0.21 \\
\hline Upper $95 \%$ of mean & 0.31 & 0.30 & 1.74 & 1.73 & 1.85 & 1.86 \\
\hline Lower $95 \%$ of mean & -1.39 & -1.41 & 0.01 & 0.02 & 0.98 & 1.00 \\
\hline Sample size & 30 & 30 & 30 & 30 & 30 & 30 \\
\hline One-sample t-test & & & & & 6.69 & 6.81 \\
\hline P-value & & & & & $<0.0001$ & $<0.0001$ \\
\hline
\end{tabular}


Table A-15. Descriptive statistics and tests for significant posttreatment changes based on repeated measurements for Pterygoid Vertical-A point (mm).

\begin{tabular}{|c|c|c|c|c|c|c|}
\hline \multirow[b]{2}{*}{ Variable } & \multicolumn{2}{|c|}{ End of Treatment } & \multicolumn{2}{|c|}{ Recall Examination } & \multicolumn{2}{|c|}{ Posttreatment Change } \\
\hline & $\mathrm{T} 1$ & $\mathrm{~T} 2$ & $\mathrm{~T} 1$ & $\mathrm{~T} 2$ & $\mathrm{~T} 1$ & $\mathrm{~T} 2$ \\
\hline Mean & 45.00 & 44.63 & 47.70 & 47.71 & 2.70 & 3.08 \\
\hline Standard deviation & 2.30 & 3.48 & 2.82 & 2.82 & 1.61 & 2.22 \\
\hline Standard error & 0.42 & 0.64 & 0.52 & 0.51 & 0.29 & 0.40 \\
\hline Upper $95 \%$ of mean & 45.86 & 45.93 & 48.76 & 48.76 & 3.30 & 3.91 \\
\hline Lower $95 \%$ of mean & 44.15 & 43.33 & 46.65 & 46.66 & 2.10 & 2.25 \\
\hline Sample size & 30 & 30 & 30 & 30 & 30 & 30 \\
\hline One-sample t-test & & & & & 9.16 & 7.61 \\
\hline P-value & & & & & $<0.0001$ & $<0.0001$ \\
\hline
\end{tabular}

Table A-16. Descriptive statistics and tests for significant posttreatment changes based on repeated measurements for Sella-A point (mm).

\begin{tabular}{|c|c|c|c|c|c|c|}
\hline \multirow[b]{2}{*}{ Variable } & \multicolumn{2}{|c|}{ End of Treatment } & \multicolumn{2}{|c|}{ Recall Examination } & \multicolumn{2}{|c|}{ Posttreatment Change } \\
\hline & $\mathrm{T} 1$ & $\mathrm{~T} 2$ & $\mathrm{~T} 1$ & $\mathrm{~T} 2$ & $\mathrm{~T} 1$ & $\mathrm{~T} 2$ \\
\hline Mean & 81.91 & 81.91 & 86.25 & 86.27 & 4.34 & 4.33 \\
\hline Standard deviation & 3.30 & 3.30 & 3.39 & 3.43 & 1.14 & 1.13 \\
\hline Standard error & 0.60 & 0.60 & 0.62 & 0.64 & 0.21 & 0.21 \\
\hline Upper $95 \%$ of mean & 83.14 & 83.14 & 87.51 & 87.58 & 4.77 & 4.75 \\
\hline Lower $95 \%$ of mean & 80.67 & 80.68 & 84.98 & 84.97 & 3.92 & 3.90 \\
\hline Sample size & 30 & 30 & 30 & 29 & 30 & 29 \\
\hline One-sample t-test & & & & & 20.95 & 20.68 \\
\hline P-value & & & & & $<0.0001$ & $<0.0001$ \\
\hline
\end{tabular}


Table A-17. Descriptive statistics and tests for significant posttreatment changes based on repeated measurements for Palatal Plane-Sella-Nasion $\left({ }^{\circ}\right)$.

\begin{tabular}{|c|c|c|c|c|c|c|}
\hline \multirow[b]{2}{*}{ Variable } & \multicolumn{2}{|c|}{ End of Treatment } & \multicolumn{2}{|c|}{$\underline{\text { Recall Examination }}$} & \multicolumn{2}{|c|}{ Posttreatment Change } \\
\hline & $\mathrm{T} 1$ & $\mathrm{~T} 2$ & $\mathrm{~T} 1$ & $\mathrm{~T} 2$ & $\mathrm{~T} 1$ & $\mathrm{~T} 2$ \\
\hline Mean & 7.13 & 7.14 & 7.58 & 7.61 & 0.45 & 0.47 \\
\hline Standard deviation & 2.95 & 2.93 & 3.15 & 3.15 & 1.84 & 1.82 \\
\hline Standard error & 0.54 & 0.53 & 0.58 & 0.58 & 0.34 & 0.33 \\
\hline Upper $95 \%$ of mean & 8.24 & 8.23 & 8.76 & 8.79 & 1.14 & 1.15 \\
\hline Lower $95 \%$ of mean & 6.03 & 6.04 & 6.41 & 6.43 & -0.24 & -0.21 \\
\hline Sample size & 30 & 30 & 30 & 30 & 30 & 30 \\
\hline One-sample t-test & & & & & 1.34 & 1.43 \\
\hline P-value & & & & & 0.1899 & 0.1644 \\
\hline
\end{tabular}

Table A-18. Descriptive statistics and tests for significant posttreatment changes based on repeated measurements for Sella-Nasion-B Point Angle $\left({ }^{\circ}\right)$.

\begin{tabular}{|c|c|c|c|c|c|c|}
\hline \multirow[b]{2}{*}{ Variable } & \multicolumn{2}{|c|}{ End of Treatment } & \multicolumn{2}{|c|}{$\underline{\text { Recall Examination }}$} & \multicolumn{2}{|c|}{$\underline{\text { Posttreatment Change }}$} \\
\hline & $\mathrm{T} 1$ & $\mathrm{~T} 2$ & $\mathrm{~T} 1$ & $\overline{\mathrm{T} 2}$ & $\mathrm{~T} 1$ & $\mathrm{~T} 2$ \\
\hline Mean & 76.38 & 76.34 & 77.15 & 77.18 & 0.77 & 0.84 \\
\hline Standard deviation & 2.98 & 2.96 & 3.03 & 3.04 & 0.26 & 0.32 \\
\hline Standard error & 0.54 & 0.54 & 0.55 & 0.56 & 0.05 & 0.06 \\
\hline Upper $95 \%$ of mean & 77.49 & 77.45 & 78.28 & 78.32 & 0.87 & 0.96 \\
\hline Lower $95 \%$ of mean & 75.27 & 75.24 & 76.02 & 76.05 & 0.67 & 0.72 \\
\hline Sample size & 30 & 30 & 30 & 30 & 30 & 30 \\
\hline One-sample t-test & & & & & 15.97 & 14.28 \\
\hline P-value & & & & & $<0.0001$ & $<0.0001$ \\
\hline
\end{tabular}


Table A-19. Descriptive statistics and tests for significant posttreatment changes based on repeated measurements for Nasion Perpendicular-B Point (mm).

\begin{tabular}{|c|c|c|c|c|c|c|}
\hline \multirow[b]{2}{*}{ Variable } & \multicolumn{2}{|c|}{ End of Treatment } & \multicolumn{2}{|c|}{ Recall Examination } & \multicolumn{2}{|c|}{ Posttreatment Change } \\
\hline & $\mathrm{T} 1$ & $\mathrm{~T} 2$ & $\mathrm{~T} 1$ & $\mathrm{~T} 2$ & $\mathrm{~T} 1$ & $\mathrm{~T} 2$ \\
\hline Mean & -5.82 & -5.84 & -3.12 & -3.14 & 2.70 & 2.71 \\
\hline Standard deviation & 3.58 & 3.61 & 3.06 & 3.08 & 2.06 & 2.06 \\
\hline Standard error & 0.65 & 0.66 & 0.56 & 0.56 & 0.38 & 0.38 \\
\hline Upper $95 \%$ of mean & -4.48 & -4.50 & -1.98 & -1.99 & 3.47 & 3.48 \\
\hline Lower $95 \%$ of mean & -7.16 & -7.19 & -4.27 & -4.29 & 1.93 & 1.94 \\
\hline Sample size & 30 & 30 & 30 & 30 & 30 & 30 \\
\hline One-sample t-test & & & & & 7.16 & 7.19 \\
\hline P-value & & & & & $<0.0001$ & $<0.0001$ \\
\hline
\end{tabular}

Table A-20. Descriptive statistics and tests for significant posttreatment changes based on repeated measurements for Pterygoid Vertical-B point (mm).

\begin{tabular}{|c|c|c|c|c|c|c|}
\hline \multirow[b]{2}{*}{ Variable } & \multicolumn{2}{|c|}{ End of Treatment } & \multicolumn{2}{|c|}{$\underline{\text { Recall Examination }}$} & \multicolumn{2}{|c|}{$\underline{\text { Posttreatment Change }}$} \\
\hline & $\mathrm{T} 1$ & $\mathrm{~T} 2$ & $\mathrm{~T} 1$ & $\mathrm{~T} 2$ & $\mathrm{~T} 1$ & $\mathrm{~T} 2$ \\
\hline Mean & 44.19 & 44.23 & 46.63 & 46.64 & 2.44 & 2.41 \\
\hline Standard deviation & 2.83 & 2.86 & 2.97 & 2.99 & 0.49 & 0.50 \\
\hline Standard error & 0.52 & 0.52 & 0.54 & 0.55 & 0.09 & 0.09 \\
\hline Upper $95 \%$ of mean & 45.25 & 45.30 & 47.74 & 47.75 & 2.62 & 2.59 \\
\hline Lower $95 \%$ of mean & 43.13 & 43.16 & 45.52 & 45.52 & 2.26 & 2.22 \\
\hline Sample size & 30 & 30 & 30 & 30 & 30 & 30 \\
\hline One-sample t-test & & & & & 27.53 & 26.24 \\
\hline P-value & & & & & $<0.0001$ & $<0.0001$ \\
\hline
\end{tabular}


Table A-21. Descriptive statistics and tests for significant posttreatment changes based on repeated measurements for Condylion-Gnathion ( $\mathrm{mm})$.

\begin{tabular}{|c|c|c|c|c|c|c|}
\hline \multirow[b]{2}{*}{ Variable } & \multicolumn{2}{|c|}{ End of Treatment } & \multicolumn{2}{|c|}{$\underline{\text { Recall Examination }}$} & \multicolumn{2}{|c|}{ Posttreatment Change } \\
\hline & $\mathrm{T} 1$ & $\mathrm{~T} 2$ & $\mathrm{~T} 1$ & $\mathrm{~T} 2$ & $\mathrm{~T} 1$ & $\mathrm{~T} 2$ \\
\hline Mean & 114.25 & 114.22 & 120.83 & 120.83 & 6.59 & 6.61 \\
\hline Standard deviation & 5.35 & 5.34 & 4.79 & 4.78 & 1.91 & 1.88 \\
\hline Standard error & 0.98 & 0.98 & 0.87 & 0.87 & 0.35 & 0.34 \\
\hline Upper $95 \%$ of mean & 116.25 & 116.21 & 122.62 & 122.62 & 7.30 & 7.32 \\
\hline Lower $95 \%$ of mean & 112.25 & 112.22 & 119.04 & 119.05 & 5.87 & 5.91 \\
\hline Sample size & 30 & 30 & 30 & 30 & 30 & 30 \\
\hline One-sample t-test & & & & & 18.91 & 19.23 \\
\hline P-value & & & & & $<0.0001$ & $<0.0001$ \\
\hline
\end{tabular}

Table A-22. Descriptive statistics and tests for significant posttreatment changes based on repeated measurements for Condylion-Gonion ( $\mathrm{mm}$ ).

\begin{tabular}{|c|c|c|c|c|c|c|}
\hline \multirow[b]{2}{*}{ Variable } & \multicolumn{2}{|c|}{ End of Treatment } & \multicolumn{2}{|c|}{ Recall Examination } & \multicolumn{2}{|c|}{ Posttreatment Change } \\
\hline & $\mathrm{T} 1$ & $\mathrm{~T} 2$ & $\mathrm{~T} 1$ & $\mathrm{~T} 2$ & $\mathrm{~T} 1$ & $\mathrm{~T} 2$ \\
\hline Mean & 60.41 & 60.43 & 65.72 & 65.72 & 5.31 & 5.29 \\
\hline Standard deviation & 4.82 & 4.82 & 5.14 & 5.12 & 2.97 & 2.95 \\
\hline Standard error & 0.88 & 0.88 & 0.94 & 0.94 & 0.54 & 0.54 \\
\hline Upper $95 \%$ of mean & 62.20 & 62.23 & 67.64 & 67.63 & 6.42 & 6.39 \\
\hline Lower $95 \%$ of mean & 58.61 & 58.63 & 63.80 & 63.80 & 4.20 & 4.19 \\
\hline Sample size & 30 & 30 & 30 & 30 & 30 & 30 \\
\hline One-sample t-test & & & & & 9.80 & 9.83 \\
\hline P-value & & & & & $<0.0001$ & $<0.0001$ \\
\hline
\end{tabular}


Table A-23. Descriptive statistics and tests for significant posttreatment changes based on repeated measurements for Gonion-Pogonion (mm).

\begin{tabular}{|c|c|c|c|c|c|c|}
\hline \multirow[b]{2}{*}{ Variable } & \multicolumn{2}{|c|}{ End of Treatment } & \multicolumn{2}{|c|}{$\underline{\text { Recall Examination }}$} & \multicolumn{2}{|c|}{ Posttreatment Change } \\
\hline & $\mathrm{T} 1$ & $\mathrm{~T} 2$ & $\mathrm{~T} 1$ & $\mathrm{~T} 2$ & $\mathrm{~T} 1$ & $\mathrm{~T} 2$ \\
\hline Mean & 76.16 & 76.14 & 80.67 & 80.70 & 4.51 & 4.56 \\
\hline Standard deviation & 4.97 & 4.99 & 4.53 & 4.53 & 2.83 & 2.84 \\
\hline Standard error & 0.91 & 0.91 & 0.83 & 0.83 & 0.52 & 0.52 \\
\hline Upper $95 \%$ of mean & 78.02 & 78.01 & 82.36 & 82.39 & 5.57 & 5.62 \\
\hline Lower $95 \%$ of mean & 74.31 & 74.28 & 78.98 & 79.01 & 3.45 & 3.49 \\
\hline Sample size & 30 & 30 & 30 & 30 & 30 & 30 \\
\hline One-sample t-test & & & & & 8.72 & 8.78 \\
\hline P-value & & & & & $<0.0001$ & $<0.0001$ \\
\hline
\end{tabular}

Table A-24. Descriptive statistics and tests for significant posttreatment changes based on repeated measurements for $\mathrm{Y}$-Axis $\left({ }^{\circ}\right)$.

\begin{tabular}{|c|c|c|c|c|c|c|}
\hline \multirow[b]{2}{*}{ Variable } & \multicolumn{2}{|c|}{ End of Treatment } & \multicolumn{2}{|c|}{ Recall Examination } & \multicolumn{2}{|c|}{ Posttreatment Change } \\
\hline & $\mathrm{T} 1$ & $\mathrm{~T} 2$ & $\mathrm{~T} 1$ & $\mathrm{~T} 2$ & $\mathrm{~T} 1$ & $\mathrm{~T} 2$ \\
\hline Mean & 57.22 & 57.23 & 56.72 & 56.77 & -0.50 & -0.46 \\
\hline Standard deviation & 3.89 & 3.91 & 3.90 & 3.96 & 1.31 & 1.28 \\
\hline Standard error & 0.71 & 0.71 & 0.71 & 0.72 & 0.24 & 0.23 \\
\hline Upper $95 \%$ of mean & 58.67 & 58.69 & 58.18 & 58.25 & -0.01 & 0.02 \\
\hline Lower $95 \%$ of mean & 55.77 & 55.77 & 55.26 & 55.29 & -0.99 & -0.94 \\
\hline Sample size & 30 & 30 & 30 & 30 & 30 & 30 \\
\hline One-sample t-test & & & & & -2.09 & -1.96 \\
\hline P-value & & & & & 0.0456 & 0.0592 \\
\hline
\end{tabular}


Table A-25. Descriptive statistics and tests for significant posttreatment changes based on repeated measurements for Nasion Perpendicular-Pogonion (mm).

\begin{tabular}{|c|c|c|c|c|c|c|}
\hline \multirow[b]{2}{*}{ Variable } & \multicolumn{2}{|c|}{ End of Treatment } & \multicolumn{2}{|c|}{$\underline{\text { Recall Examination }}$} & \multicolumn{2}{|c|}{ Posttreatment Change } \\
\hline & $\mathrm{T} 1$ & $\mathrm{~T} 2$ & $\mathrm{~T} 1$ & $\mathrm{~T} 2$ & $\mathrm{~T} 1$ & $\mathrm{~T} 2$ \\
\hline Mean & -3.60 & -3.62 & -1.59 & -1.59 & 2.00 & 2.03 \\
\hline Standard deviation & 4.49 & 4.49 & 4.37 & 4.39 & 2.38 & 2.40 \\
\hline Standard error & 0.82 & 0.82 & 0.80 & 0.80 & 0.44 & 0.44 \\
\hline Upper $95 \%$ of mean & -1.92 & -1.94 & 0.04 & 0.05 & 2.89 & 2.93 \\
\hline Lower $95 \%$ of mean & -5.27 & -5.30 & -3.23 & -3.23 & 1.11 & 1.14 \\
\hline Sample size & 30 & 30 & 30 & 30 & 30 & 30 \\
\hline One-sample t-test & & & & & 4.60 & 4.64 \\
\hline P-value & & & & & $<0.0001$ & $<0.0001$ \\
\hline
\end{tabular}

Table A-26. Descriptive statistics and tests for significant posttreatment changes based on repeated measurements for A point-Nasion-B point Angle $\left(^{\circ}\right)$.

\begin{tabular}{|c|c|c|c|c|c|c|}
\hline \multirow[b]{2}{*}{ Variable } & \multicolumn{2}{|c|}{ End of Treatment } & \multicolumn{2}{|c|}{$\underline{\text { Recall Examination }}$} & \multicolumn{2}{|c|}{$\underline{\text { Posttreatment Change }}$} \\
\hline & $\mathrm{T} 1$ & $\mathrm{~T} 2$ & $\mathrm{~T} 1$ & $\overline{\mathrm{T} 2}$ & $\mathrm{~T} 1$ & $\mathrm{~T} 2$ \\
\hline Mean & 3.51 & 3.54 & 3.21 & 3.18 & -0.30 & -0.36 \\
\hline Standard deviation & 1.94 & 1.96 & 1.99 & 1.98 & 0.28 & 0.34 \\
\hline Standard error & 0.35 & 0.36 & 0.36 & 0.36 & 0.05 & 0.06 \\
\hline Upper $95 \%$ of mean & 4.24 & 4.27 & 3.96 & 3.92 & -0.19 & -0.23 \\
\hline Lower $95 \%$ of mean & 2.79 & 2.81 & 2.47 & 2.44 & -0.41 & -0.49 \\
\hline Sample size & 30 & 30 & 30 & 30 & 30 & 30 \\
\hline One-sample t-test & & & & & -5.81 & -5.85 \\
\hline P-value & & & & & $<0.0001$ & $<0.0001$ \\
\hline
\end{tabular}


Table A-27. Descriptive statistics and tests for significant posttreatment changes based on repeated measurements for 'Wits' Appraisal (mm).

\begin{tabular}{|c|c|c|c|c|c|c|}
\hline \multirow[b]{2}{*}{ Variable } & \multicolumn{2}{|c|}{ End of Treatment } & \multicolumn{2}{|c|}{$\underline{\text { Recall Examination }}$} & \multicolumn{2}{|c|}{ Posttreatment Change } \\
\hline & $\mathrm{T} 1$ & $\mathrm{~T} 2$ & $\mathrm{~T} 1$ & $\mathrm{~T} 2$ & $\mathrm{~T} 1$ & $\mathrm{~T} 2$ \\
\hline Mean & 1.08 & 1.08 & 0.57 & 0.61 & -0.51 & -0.47 \\
\hline Standard deviation & 2.68 & 2.67 & 3.17 & 3.17 & 2.50 & 2.48 \\
\hline Standard error & 0.49 & 0.49 & 0.58 & 0.58 & 0.46 & 0.45 \\
\hline Upper $95 \%$ of mean & 2.08 & 2.08 & 1.76 & 1.80 & 0.43 & 0.46 \\
\hline Lower $95 \%$ of mean & 0.08 & 0.08 & -0.61 & -0.57 & -1.44 & -1.39 \\
\hline Sample size & 30 & 30 & 30 & 30 & 30 & 30 \\
\hline One-sample t-test & & & & & -1.11 & -1.03 \\
\hline P-value & & & & & 0.2756 & 0.3112 \\
\hline
\end{tabular}

Table A-28. Descriptive statistics and tests for significant posttreatment changes based on repeated measurements for Frankfort Horizontal-Mandibular Plane Angle $\left(^{\circ}\right)$.

\begin{tabular}{|c|c|c|c|c|c|c|}
\hline \multirow[b]{2}{*}{ Variable } & \multicolumn{2}{|c|}{ End of Treatment } & \multicolumn{2}{|c|}{ Recall Examination } & \multicolumn{2}{|c|}{ Posttreatment Change } \\
\hline & $\mathrm{T} 1$ & $\mathrm{~T} 2$ & $\mathrm{~T} 1$ & $\mathrm{~T} 2$ & $\mathrm{~T} 1$ & $\mathrm{~T} 2$ \\
\hline Mean & 20.60 & 20.60 & 20.06 & 20.09 & -0.54 & -0.51 \\
\hline Standard deviation & 6.28 & 6.26 & 6.69 & 6.67 & 2.35 & 2.40 \\
\hline Standard error & 1.15 & 1.14 & 1.22 & 1.22 & 0.43 & 0.44 \\
\hline Upper $95 \%$ of mean & 22.94 & 22.94 & 22.56 & 22.58 & 0.34 & 0.39 \\
\hline Lower $95 \%$ of mean & 18.26 & 18.26 & 17.57 & 17.60 & -1.42 & -1.41 \\
\hline Sample size & 30 & 30 & 30 & 30 & 30 & 30 \\
\hline One-sample t-test & & & & & -1.25 & -1.16 \\
\hline P-value & & & & & 0.2218 & 0.2548 \\
\hline
\end{tabular}


Table A-29. Descriptive statistics and tests for significant posttreatment changes based on repeated measurements for Nasion-A point-Pogonion Angle $\left(^{\circ}\right)$.

\begin{tabular}{|c|c|c|c|c|c|c|}
\hline \multirow[b]{2}{*}{ Variable } & \multicolumn{2}{|c|}{ End of Treatment } & \multicolumn{2}{|c|}{$\underline{\text { Recall Examination }}$} & \multicolumn{2}{|c|}{ Posttreatment Change } \\
\hline & $\mathrm{T} 1$ & $\mathrm{~T} 2$ & $\mathrm{~T} 1$ & $\mathrm{~T} 2$ & $\mathrm{~T} 1$ & $\mathrm{~T} 2$ \\
\hline Mean & 3.47 & 3.46 & 3.94 & 3.97 & 0.47 & 0.50 \\
\hline Standard deviation & 4.82 & 4.84 & 4.63 & 4.60 & 2.00 & 2.03 \\
\hline Standard error & 0.88 & 0.88 & 0.85 & 0.84 & 0.37 & 0.37 \\
\hline Upper $95 \%$ of mean & 5.27 & 5.27 & 5.67 & 5.68 & 1.22 & 1.26 \\
\hline Lower $95 \%$ of mean & 1.67 & 1.65 & 2.21 & 2.25 & -0.27 & -0.26 \\
\hline Sample size & 30 & 30 & 30 & 30 & 30 & 30 \\
\hline One-sample t-test & & & & & 1.29 & 1.36 \\
\hline P-value & & & & & 0.2058 & 0.1856 \\
\hline
\end{tabular}

Table A-30. Descriptive statistics and tests for significant posttreatment changes based on repeated measurements for Frankfort Horizontal-Nasion-Pogonion Angle $\left(^{\circ}\right)$.

\begin{tabular}{|c|c|c|c|c|c|c|}
\hline \multirow[b]{2}{*}{ Variable } & \multicolumn{2}{|c|}{ End of Treatment } & \multicolumn{2}{|c|}{ Recall Examination } & \multicolumn{2}{|c|}{ Posttreatment Change } \\
\hline & $\mathrm{T} 1$ & $\mathrm{~T} 2$ & $\mathrm{~T} 1$ & $\mathrm{~T} 2$ & $\mathrm{~T} 1$ & $\mathrm{~T} 2$ \\
\hline Mean & 87.66 & 87.74 & 88.98 & 88.99 & 1.32 & 1.25 \\
\hline Standard deviation & 3.20 & 2.97 & 2.94 & 2.93 & 1.45 & 1.41 \\
\hline Standard error & 0.58 & 0.54 & 0.54 & 0.53 & 0.26 & 0.26 \\
\hline Upper $95 \%$ of mean & 88.85 & 88.85 & 90.08 & 90.08 & 1.86 & 1.78 \\
\hline Lower $95 \%$ of mean & 86.47 & 86.63 & 87.88 & 87.89 & 0.78 & 0.72 \\
\hline Sample size & 30 & 30 & 30 & 30 & 30 & 30 \\
\hline One-sample t-test & & & & & 4.99 & 4.85 \\
\hline P-value & & & & & $<0.0001$ & $<0.0001$ \\
\hline
\end{tabular}


Table A-31. Descriptive statistics and tests for significant posttreatment changes based on repeated measurements for Interincisal Angle $\left(^{\circ}\right)$.

\begin{tabular}{|c|c|c|c|c|c|c|}
\hline \multirow[b]{2}{*}{ Variable } & \multicolumn{2}{|c|}{ End of Treatment } & \multicolumn{2}{|c|}{ Recall Examination } & \multicolumn{2}{|c|}{ Posttreatment Change } \\
\hline & $\mathrm{T} 1$ & $\mathrm{~T} 2$ & $\mathrm{~T} 1$ & $\mathrm{~T} 2$ & $\mathrm{~T} 1$ & $\mathrm{~T} 2$ \\
\hline Mean & 118.68 & 118.51 & 125.24 & 125.26 & 6.56 & 6.75 \\
\hline Standard deviation & 6.98 & 6.68 & 7.59 & 7.58 & 6.25 & 6.29 \\
\hline Standard error & 1.27 & 1.22 & 1.39 & 1.38 & 1.14 & 1.15 \\
\hline Upper $95 \%$ of mean & 121.28 & 121.00 & 128.07 & 128.09 & 8.90 & 9.10 \\
\hline Lower $95 \%$ of mean & 116.07 & 116.02 & 122.41 & 122.43 & 4.23 & 4.40 \\
\hline Sample size & 30 & 30 & 30 & 30 & 30 & 30 \\
\hline One-sample t-test & & & & & 5.75 & 5.88 \\
\hline P-value & & & & & $<0.0001$ & $<0.0001$ \\
\hline
\end{tabular}

Table A-32. Descriptive statistics and tests for significant posttreatment changes based on repeated measurements for Upper 1/Sella-Nasion Angle $\left(^{\circ}\right)$.

\begin{tabular}{|c|c|c|c|c|c|c|}
\hline \multirow[b]{2}{*}{ Variable } & \multicolumn{2}{|c|}{ End of Treatment } & \multicolumn{2}{|c|}{ Recall Examination } & \multicolumn{2}{|c|}{ Posttreatment Change } \\
\hline & $\mathrm{T} 1$ & $\mathrm{~T} 2$ & $\mathrm{~T} 1$ & $\mathrm{~T} 2$ & $\mathrm{~T} 1$ & $\mathrm{~T} 2$ \\
\hline Mean & 107.21 & 107.17 & 104.11 & 104.17 & -3.11 & -3.00 \\
\hline Standard deviation & 5.00 & 5.07 & 7.31 & 7.34 & 5.08 & 5.13 \\
\hline Standard error & 0.91 & 0.93 & 1.33 & 1.34 & 0.93 & 0.94 \\
\hline Upper $95 \%$ of mean & 109.08 & 109.06 & 106.83 & 106.91 & -1.21 & -1.08 \\
\hline Lower $95 \%$ of mean & 105.35 & 105.27 & 101.38 & 101.43 & -5.01 & -4.91 \\
\hline Sample size & 30 & 30 & 30 & 30 & 30 & 30 \\
\hline One-sample t-test & & & & & -3.35 & -3.20 \\
\hline P-value & & & & & 0.0023 & 0.0033 \\
\hline
\end{tabular}


Table A-33. Descriptive statistics and tests for significant posttreatment changes based on repeated measurements for Upper 1/Nasion-A Point Angle $\left(^{\circ}\right)$.

\begin{tabular}{|c|c|c|c|c|c|c|}
\hline \multirow[b]{2}{*}{ Variable } & \multicolumn{2}{|c|}{ End of Treatment } & \multicolumn{2}{|c|}{$\underline{\text { Recall Examination }}$} & \multicolumn{2}{|c|}{ Posttreatment Change } \\
\hline & $\mathrm{T} 1$ & $\mathrm{~T} 2$ & $\mathrm{~T} 1$ & $\overline{\mathrm{T} 2}$ & $\mathrm{~T} 1$ & $\mathrm{~T} 2$ \\
\hline Mean & 27.29 & 27.22 & 23.74 & 23.70 & -3.55 & -3.52 \\
\hline Standard deviation & 4.94 & 4.88 & 6.98 & 6.97 & 5.06 & 5.04 \\
\hline Standard error & 0.90 & 0.89 & 1.27 & 1.27 & 0.92 & 0.92 \\
\hline Upper $95 \%$ of mean & 29.13 & 29.05 & 26.34 & 26.31 & -1.66 & -1.64 \\
\hline Lower $95 \%$ of mean & 25.44 & 25.40 & 21.13 & 21.10 & -5.44 & -5.40 \\
\hline Sample size & 30 & 30 & 30 & 30 & 30 & 30 \\
\hline One-sample t-test & & & & & -3.84 & -3.83 \\
\hline P-value & & & & & 0.0006 & 0.0006 \\
\hline
\end{tabular}

Table A-34. Descriptive statistics and tests for significant posttreatment changes based on repeated measurements for Upper 1/Nasion-A point Distance (mm).

\begin{tabular}{|c|c|c|c|c|c|c|}
\hline \multirow[b]{2}{*}{ Variable } & \multicolumn{2}{|c|}{ End of Treatment } & \multicolumn{2}{|c|}{ Recall Examination } & \multicolumn{2}{|c|}{ Posttreatment Change } \\
\hline & $\mathrm{T} 1$ & $\mathrm{~T} 2$ & $\mathrm{~T} 1$ & $\mathrm{~T} 2$ & $\mathrm{~T} 1$ & $\mathrm{~T} 2$ \\
\hline Mean & 3.10 & 3.13 & 2.87 & 2.89 & -0.23 & -0.24 \\
\hline Standard deviation & 2.22 & 2.22 & 2.22 & 2.23 & 1.65 & 1.66 \\
\hline Standard error & 0.41 & 0.41 & 0.40 & 0.41 & 0.30 & 0.30 \\
\hline Upper $95 \%$ of mean & 3.93 & 3.96 & 3.70 & 3.72 & 0.38 & 0.38 \\
\hline Lower $95 \%$ of mean & 2.27 & 2.30 & 2.04 & 2.06 & -0.85 & -0.86 \\
\hline Sample size & 30 & 30 & 30 & 30 & 30 & 30 \\
\hline One-sample t-test & & & & & -0.78 & -0.80 \\
\hline P-value & & & & & 0.4438 & 0.4277 \\
\hline
\end{tabular}


Table A-35. Descriptive statistics and tests for significant posttreatment changes based on repeated measurements for Lower 1/Nasion-B Point Angle $\left(^{\circ}\right)$.

\begin{tabular}{|c|c|c|c|c|c|c|}
\hline \multirow[b]{2}{*}{ Variable } & \multicolumn{2}{|c|}{ End of Treatment } & \multicolumn{2}{|c|}{$\underline{\text { Recall Examination }}$} & \multicolumn{2}{|c|}{ Posttreatment Change } \\
\hline & $\mathrm{T} 1$ & $\mathrm{~T} 2$ & $\mathrm{~T} 1$ & $\mathrm{~T} 2$ & $\mathrm{~T} 1$ & $\mathrm{~T} 2$ \\
\hline Mean & 30.67 & 30.27 & 27.79 & 27.80 & -2.88 & -2.48 \\
\hline Standard deviation & 5.43 & 5.37 & 4.89 & 4.89 & 5.50 & 4.98 \\
\hline Standard error & 0.99 & 0.98 & 0.89 & 0.89 & 1.00 & 0.91 \\
\hline Upper $95 \%$ of mean & 32.70 & 32.28 & 29.62 & 29.62 & -0.83 & -0.62 \\
\hline Lower $95 \%$ of mean & 28.64 & 28.27 & 25.97 & 25.97 & -4.93 & -4.33 \\
\hline Sample size & 30 & 30 & 30 & 30 & 30 & 30 \\
\hline One-sample t-test & & & & & -2.87 & -2.73 \\
\hline P-value & & & & & 0.0076 & 0.0108 \\
\hline
\end{tabular}

Table A-36. Descriptive statistics and tests for significant posttreatment changes based on repeated measurements for Lower 1 /Nasion-B point Distance (mm).

\begin{tabular}{|c|c|c|c|c|c|c|}
\hline \multirow[b]{2}{*}{ Variable } & \multicolumn{2}{|c|}{ End of Treatment } & \multicolumn{2}{|c|}{ Recall Examination } & \multicolumn{2}{|c|}{ Posttreatment Change } \\
\hline & $\mathrm{T} 1$ & $\mathrm{~T} 2$ & $\mathrm{~T} 1$ & $\mathrm{~T} 2$ & $\mathrm{~T} 1$ & $\mathrm{~T} 2$ \\
\hline Mean & 4.75 & 4.79 & 3.42 & 3.43 & -1.33 & -1.36 \\
\hline Standard deviation & 2.09 & 2.10 & 1.45 & 1.41 & 1.56 & 1.56 \\
\hline Standard error & 0.38 & 0.38 & 0.27 & 0.26 & 0.28 & 0.28 \\
\hline Upper $95 \%$ of mean & 5.53 & 5.58 & 3.96 & 3.95 & -0.75 & -0.78 \\
\hline Lower $95 \%$ of mean & 3.97 & 4.00 & 2.87 & 2.90 & -1.91 & -1.95 \\
\hline Sample size & 30 & 30 & 30 & 30 & 30 & 30 \\
\hline One-sample t-test & & & & & -4.68 & -4.79 \\
\hline P-value & & & & & $<0.0001$ & $<0.0001$ \\
\hline
\end{tabular}


Table A-37. Descriptive statistics and tests for significant posttreatment changes based on repeated measurements for Lower Incisor-Mandibular Plane Angle $\left(^{\circ}\right)$.

\begin{tabular}{|c|c|c|c|c|c|c|}
\hline \multirow[b]{2}{*}{ Variable } & \multicolumn{2}{|c|}{ End of Treatment } & \multicolumn{2}{|c|}{ Recall Examination } & \multicolumn{2}{|c|}{ Posttreatment Change } \\
\hline & $\mathrm{T} 1$ & $\mathrm{~T} 2$ & $\mathrm{~T} 1$ & $\mathrm{~T} 2$ & $\mathrm{~T} 1$ & $\mathrm{~T} 2$ \\
\hline Mean & 103.69 & 103.67 & 99.54 & 99.57 & -4.15 & -4.11 \\
\hline Standard deviation & 7.59 & 7.65 & 7.33 & 7.30 & 6.33 & 6.35 \\
\hline Standard error & 1.39 & 1.40 & 1.34 & 1.33 & 1.16 & 1.16 \\
\hline Upper $95 \%$ of mean & 106.53 & 106.53 & 102.28 & 102.29 & -1.79 & -1.74 \\
\hline Lower $95 \%$ of mean & 100.86 & 100.82 & 96.81 & 96.84 & -6.51 & -6.48 \\
\hline Sample size & 30 & 30 & 30 & 30 & 30 & 30 \\
\hline One-sample t-test & & & & & -3.59 & -3.54 \\
\hline P-value & & & & & 0.0012 & 0.0014 \\
\hline
\end{tabular}

Table A-38. Descriptive statistics and tests for significant posttreatment changes based on repeated measurements for Frankfort Horizontal-Mandibular Incisor Angle $\left(^{\circ}\right)$.

\begin{tabular}{|c|c|c|c|c|c|c|}
\hline \multirow[b]{2}{*}{ Variable } & \multicolumn{2}{|c|}{ End of Treatment } & \multicolumn{2}{|c|}{ Recall Examination } & \multicolumn{2}{|c|}{ Posttreatment Change } \\
\hline & $\mathrm{T} 1$ & $\mathrm{~T} 2$ & $\mathrm{~T} 1$ & $\mathrm{~T} 2$ & $\mathrm{~T} 1$ & $\mathrm{~T} 2$ \\
\hline Mean & 55.39 & 55.40 & 60.00 & 60.03 & 4.61 & 4.63 \\
\hline Standard deviation & 6.41 & 6.41 & 4.87 & 4.86 & 6.06 & 6.11 \\
\hline Standard error & 1.17 & 1.17 & 0.89 & 0.89 & 1.11 & 1.12 \\
\hline Upper $95 \%$ of mean & 57.79 & 57.79 & 61.82 & 61.84 & 6.87 & 6.91 \\
\hline Lower $95 \%$ of mean & 53.00 & 53.01 & 58.18 & 58.22 & 2.34 & 2.35 \\
\hline Sample size & 30 & 30 & 30 & 30 & 30 & 30 \\
\hline One-sample t-test & & & & & 4.16 & 4.15 \\
\hline P-value & & & & & 0.0003 & 0.0003 \\
\hline
\end{tabular}


Table A-39. Descriptive statistics and tests for significant posttreatment changes based on repeated measurements for Upper 1/A Point.-Pogonion (mm).

\begin{tabular}{|c|c|c|c|c|c|c|}
\hline \multirow[b]{2}{*}{ Variable } & \multicolumn{2}{|c|}{ End of Treatment } & \multicolumn{2}{|c|}{ Recall Examination } & \multicolumn{2}{|c|}{ Posttreatment Change } \\
\hline & $\mathrm{T} 1$ & $\mathrm{~T} 2$ & $\mathrm{~T} 1$ & $\mathrm{~T} 2$ & $\mathrm{~T} 1$ & $\mathrm{~T} 2$ \\
\hline Mean & 4.53 & 4.57 & 4.55 & 4.57 & 0.02 & 0.00 \\
\hline Standard deviation & 1.76 & 1.75 & 1.59 & 1.60 & 1.15 & 1.14 \\
\hline Standard error & 0.32 & 0.32 & 0.29 & 0.29 & 0.21 & 0.21 \\
\hline Upper $95 \%$ of mean & 5.19 & 5.22 & 5.15 & 5.16 & 0.45 & 0.43 \\
\hline Lower $95 \%$ of mean & 3.88 & 3.91 & 3.96 & 3.97 & -0.41 & -0.43 \\
\hline Sample size & 30 & 30 & 30 & 30 & 30 & 30 \\
\hline One-sample t-test & & & & & 0.10 & 0.00 \\
\hline P-value & & & & & 0.9248 & 1.0000 \\
\hline
\end{tabular}

Table A-40. Descriptive statistics and tests for significant posttreatment changes based on repeated measurements for Lower 1/A Point-Pogonion (mm).

\begin{tabular}{|c|c|c|c|c|c|c|}
\hline \multirow[b]{2}{*}{ Variable } & \multicolumn{2}{|c|}{ End of Treatment } & \multicolumn{2}{|c|}{$\underline{\text { Recall Examination }}$} & \multicolumn{2}{|c|}{ Posttreatment Change } \\
\hline & $\mathrm{T} 1$ & $\mathrm{~T} 2$ & $\mathrm{~T} 1$ & $\mathrm{~T} 2$ & $\mathrm{~T} 1$ & $\mathrm{~T} 2$ \\
\hline Mean & 1.78 & 1.74 & 0.85 & 0.88 & -0.93 & -0.86 \\
\hline Standard deviation & 1.82 & 1.81 & 1.33 & 1.33 & 1.26 & 1.27 \\
\hline Standard error & 0.33 & 0.33 & 0.24 & 0.24 & 0.23 & 0.23 \\
\hline Upper $95 \%$ of mean & 2.46 & 2.42 & 1.35 & 1.38 & -0.46 & -0.39 \\
\hline Lower $95 \%$ of mean & 1.10 & 1.07 & 0.36 & 0.38 & -1.40 & -1.34 \\
\hline Sample size & 30 & 30 & 30 & 30 & 30 & 30 \\
\hline One-sample t-test & & & & & -4.05 & -3.72 \\
\hline P-value & & & & & 0.0004 & 0.0008 \\
\hline
\end{tabular}


Table A-41. Descriptive statistics and tests for significant posttreatment changes based on repeated measurements for Down's Occlusal Plane-Frankfort Horizontal Angle $\left(^{\circ}\right)$.

\begin{tabular}{|c|c|c|c|c|c|c|}
\hline \multirow[b]{2}{*}{ Variable } & \multicolumn{2}{|c|}{ End of Treatment } & \multicolumn{2}{|c|}{$\underline{\text { Recall Examination }}$} & \multicolumn{2}{|c|}{ Posttreatment Change } \\
\hline & $\mathrm{T} 1$ & $\mathrm{~T} 2$ & $\mathrm{~T} 1$ & $\mathrm{~T} 2$ & $\mathrm{~T} 1$ & $\mathrm{~T} 2$ \\
\hline Mean & 6.29 & 6.30 & 4.41 & 4.38 & -1.87 & -1.84 \\
\hline Standard deviation & 2.95 & 2.96 & 3.81 & 4.47 & 3.09 & 3.09 \\
\hline Standard error & 0.54 & 0.54 & 0.70 & 3.82 & 0.56 & 0.56 \\
\hline Upper $95 \%$ of mean & 7.39 & 7.41 & 5.84 & 0.70 & -0.72 & -0.68 \\
\hline Lower $95 \%$ of mean & 5.19 & 5.20 & 2.99 & 5.89 & -3.03 & -2.99 \\
\hline Sample size & 30 & 30 & 30 & 3.0412549 & 30 & 30 \\
\hline One-sample t-test & & & & & -3.33 & -3.26 \\
\hline P-value & & & & & 0.0024 & 0.0029 \\
\hline
\end{tabular}

Table A-42. Descriptive statistics and tests for significant posttreatment changes based on repeated measurements for Overbite (mm).

\begin{tabular}{|c|c|c|c|c|c|c|}
\hline \multirow[b]{2}{*}{ Variable } & \multicolumn{2}{|c|}{ End of Treatment } & \multicolumn{2}{|c|}{$\underline{\text { Recall Examination }}$} & \multicolumn{2}{|c|}{$\underline{\text { Posttreatment Change }}$} \\
\hline & $\mathrm{T} 1$ & $\mathrm{~T} 2$ & $\mathrm{~T} 1$ & $\overline{\mathrm{T} 2}$ & $\mathrm{~T} 1$ & $\mathrm{~T} 2$ \\
\hline Mean & 2.12 & 2.16 & 3.02 & 3.06 & 0.90 & 0.90 \\
\hline Standard deviation & 0.95 & 0.95 & 1.31 & 1.31 & 0.84 & 0.85 \\
\hline Standard error & 0.17 & 0.17 & 0.24 & 0.24 & 0.15 & 0.15 \\
\hline Upper $95 \%$ of mean & 2.48 & 2.51 & 3.51 & 3.55 & 1.21 & 1.22 \\
\hline Lower $95 \%$ of mean & 1.77 & 1.80 & 2.53 & 2.57 & 0.59 & 0.58 \\
\hline Sample size & 30 & 30 & 30 & 30 & 30 & 30 \\
\hline One-sample t-test & & & & & 5.89 & 5.81 \\
\hline P-value & & & & & $<0.0001$ & $<0.0001$ \\
\hline
\end{tabular}


Table A-43. Descriptive statistics and tests for significant posttreatment changes based on repeated measurements for Overjet (mm).

\begin{tabular}{|c|c|c|c|c|c|c|}
\hline \multirow[b]{2}{*}{ Variable } & \multicolumn{2}{|c|}{ End of Treatment } & \multicolumn{2}{|c|}{$\underline{\text { Recall Examination }}$} & \multicolumn{2}{|c|}{ Posttreatment Change } \\
\hline & $\mathrm{T} 1$ & $\mathrm{~T} 2$ & $\mathrm{~T} 1$ & $\mathrm{~T} 2$ & $\mathrm{~T} 1$ & $\mathrm{~T} 2$ \\
\hline Mean & 2.81 & 2.77 & 3.76 & 3.78 & 0.95 & 1.00 \\
\hline Standard deviation & 0.72 & 0.69 & 0.77 & 0.78 & 1.06 & 1.05 \\
\hline Standard error & 0.13 & 0.13 & 0.14 & 0.14 & 0.19 & 0.19 \\
\hline Upper $95 \%$ of mean & 3.08 & 3.03 & 4.05 & 4.07 & 1.34 & 1.39 \\
\hline Lower $95 \%$ of mean & 2.54 & 2.51 & 3.47 & 3.49 & 0.55 & 0.61 \\
\hline Sample size & 30 & 30 & 30 & 30 & 30 & 30 \\
\hline One-sample t-test & & & & & 4.91 & 5.25 \\
\hline P-value & & & & & $<0.0001$ & $<0.0001$ \\
\hline
\end{tabular}

Table A-44. Descriptive statistics and tests for significant posttreatment changes based on repeated measurements for Lower 1-W point (mm).

\begin{tabular}{|c|c|c|c|c|c|c|}
\hline \multirow[b]{2}{*}{ Variable } & \multicolumn{2}{|c|}{ End of Treatment } & \multicolumn{2}{|c|}{ Recall Examination } & \multicolumn{2}{|c|}{ Posttreatment Change } \\
\hline & $\mathrm{T} 1$ & $\mathrm{~T} 2$ & $\mathrm{~T} 1$ & $\mathrm{~T} 2$ & $\mathrm{~T} 1$ & $\mathrm{~T} 2$ \\
\hline Mean & 9.49 & 9.53 & 9.44 & 9.44 & -0.05 & -0.09 \\
\hline Standard deviation & 1.84 & 1.87 & 1.65 & 1.65 & 1.05 & 1.03 \\
\hline Standard error & 0.34 & 0.34 & 0.30 & 0.30 & 0.19 & 0.19 \\
\hline Upper $95 \%$ of mean & 10.18 & 10.22 & 10.06 & 10.05 & 0.34 & 0.30 \\
\hline Lower $95 \%$ of mean & 8.80 & 8.83 & 8.82 & 8.82 & -0.44 & -0.48 \\
\hline Sample size & 30 & 30 & 30 & 30 & 30 & 30 \\
\hline One-sample t-test & & & & & -0.28 & -0.48 \\
\hline P-value & & & & & 0.7821 & 0.6367 \\
\hline
\end{tabular}


Table A-45. Descriptive statistics and tests for significant posttreatment changes based on repeated measurements for Distal Lower 6-W point (mm).

\begin{tabular}{|c|c|c|c|c|c|c|}
\hline \multirow[b]{2}{*}{ Variable } & \multicolumn{2}{|c|}{ End of Treatment } & \multicolumn{2}{|c|}{ Recall Examination } & \multicolumn{2}{|c|}{ Posttreatment Change } \\
\hline & $\mathrm{T} 1$ & $\mathrm{~T} 2$ & $\mathrm{~T} 1$ & $\mathrm{~T} 2$ & $\mathrm{~T} 1$ & $\mathrm{~T} 2$ \\
\hline Mean & 15.65 & 15.67 & 14.72 & 13.84 & -0.93 & -1.83 \\
\hline Standard deviation & 1.93 & 1.88 & 2.14 & 5.52 & 0.58 & 4.98 \\
\hline Standard error & 0.35 & 0.34 & 0.39 & 1.01 & 0.11 & 0.91 \\
\hline Upper $95 \%$ of mean & 16.37 & 16.37 & 15.52 & 15.90 & -0.71 & 0.03 \\
\hline Lower $95 \%$ of mean & 14.93 & 14.97 & 13.92 & 11.77 & -1.14 & -3.69 \\
\hline Sample size & 30 & 30 & 30 & 30 & 30 & 30 \\
\hline One-sample t-test & & & & & -8.75 & -2.01 \\
\hline P-value & & & & & $<0.0001$ & 0.0533 \\
\hline
\end{tabular}

Table A-46. Descriptive statistics and tests for significant posttreatment changes based on repeated measurements for Upper 1-Pterygoid Vertical (mm).

\begin{tabular}{|c|c|c|c|c|c|c|}
\hline \multirow[b]{2}{*}{ Variable } & \multicolumn{2}{|c|}{ End of Treatment } & \multicolumn{2}{|c|}{ Recall Examination } & \multicolumn{2}{|c|}{ Posttreatment Change } \\
\hline & $\mathrm{T} 1$ & $\mathrm{~T} 2$ & $\mathrm{~T} 1$ & $\mathrm{~T} 2$ & $\mathrm{~T} 1$ & $\mathrm{~T} 2$ \\
\hline Mean & 51.27 & 51.32 & 54.23 & 54.26 & 2.95 & 2.93 \\
\hline Standard deviation & 4.21 & 4.19 & 4.05 & 4.07 & 1.86 & 1.86 \\
\hline Standard error & 0.77 & 0.77 & 0.74 & 0.74 & 0.34 & 0.34 \\
\hline Upper $95 \%$ of mean & 52.85 & 52.89 & 55.74 & 55.77 & 3.65 & 3.63 \\
\hline Lower $95 \%$ of mean & 49.70 & 49.76 & 52.72 & 52.74 & 2.26 & 2.24 \\
\hline Sample size & 30 & 30 & 30 & 30 & 30 & 30 \\
\hline One-sample t-test & & & & & 8.68 & 8.65 \\
\hline P-value & & & & & $<0.0001$ & $<0.0001$ \\
\hline
\end{tabular}


Table A-47. Descriptive statistics and tests for significant posttreatment changes based on repeated measurements for Upper 6-Pterygoid Vertical (mm).

\begin{tabular}{|c|c|c|c|c|c|c|}
\hline \multirow[b]{2}{*}{ Variable } & \multicolumn{2}{|c|}{ End of Treatment } & \multicolumn{2}{|c|}{ Recall Examination } & \multicolumn{2}{|c|}{ Posttreatment Change } \\
\hline & $\mathrm{T} 1$ & $\mathrm{~T} 2$ & $\mathrm{~T} 1$ & $\mathrm{~T} 2$ & $\mathrm{~T} 1$ & $\mathrm{~T} 2$ \\
\hline Mean & 16.39 & 16.40 & 18.75 & 18.76 & 2.37 & 2.36 \\
\hline Standard deviation & 3.27 & 3.28 & 2.98 & 2.97 & 1.75 & 1.75 \\
\hline Standard error & 0.61 & 0.61 & 0.55 & 0.55 & 0.32 & 0.33 \\
\hline Upper $95 \%$ of mean & 17.63 & 17.65 & 19.88 & 19.89 & 3.03 & 3.03 \\
\hline Lower $95 \%$ of mean & 15.14 & 15.16 & 17.62 & 17.63 & 1.70 & 1.69 \\
\hline Sample size & 29 & 29 & 29 & 29 & 29 & 29 \\
\hline One-sample t-test & & & & & 7.28 & 7.25 \\
\hline P-value & & & & & $<0.0001$ & $<0.0001$ \\
\hline
\end{tabular}

Table A-48. Descriptive statistics and tests for significant posttreatment changes based on repeated measurements for Distal Lower 6-Lower 1 (mm).

\begin{tabular}{|c|c|c|c|c|c|c|}
\hline \multirow[b]{2}{*}{ Variable } & \multicolumn{2}{|c|}{ End of Treatment } & \multicolumn{2}{|c|}{ Recall Examination } & \multicolumn{2}{|c|}{ Posttreatment Change } \\
\hline & $\mathrm{T} 1$ & $\mathrm{~T} 2$ & $\mathrm{~T} 1$ & $\mathrm{~T} 2$ & $\mathrm{~T} 1$ & $\mathrm{~T} 2$ \\
\hline Mean & 35.19 & 35.18 & 33.95 & 33.93 & -1.24 & -1.25 \\
\hline Standard deviation & 1.77 & 1.75 & 1.79 & 1.79 & 0.57 & 0.58 \\
\hline Standard error & 0.32 & 0.32 & 0.33 & 0.33 & 0.10 & 0.11 \\
\hline Upper $95 \%$ of mean & 35.85 & 35.83 & 34.62 & 34.60 & -1.03 & -1.03 \\
\hline Lower $95 \%$ of mean & 34.53 & 34.53 & 33.28 & 33.26 & -1.46 & -1.46 \\
\hline Sample size & 30 & 30 & 30 & 30 & 30 & 30 \\
\hline One-sample t-test & & & & & 11.93 & 11.77 \\
\hline P-value & & & & & $<0.0001$ & $<0.0001$ \\
\hline
\end{tabular}


Table A-49. Descriptive statistics and tests for significant posttreatment changes based on repeated measurements for Distal Upper 6-Upper 1 (mm).

\begin{tabular}{|c|c|c|c|c|c|c|}
\hline \multirow[b]{2}{*}{ Variable } & \multicolumn{2}{|c|}{ End of Treatment } & \multicolumn{2}{|c|}{ Recall Examination } & \multicolumn{2}{|c|}{ Posttreatment Change } \\
\hline & $\mathrm{T} 1$ & $\mathrm{~T} 2$ & $\mathrm{~T} 1$ & $\mathrm{~T} 2$ & $\mathrm{~T} 1$ & $\mathrm{~T} 2$ \\
\hline Mean & 40.71 & 40.71 & 38.98 & 38.99 & -1.73 & -1.72 \\
\hline Standard deviation & 2.53 & 2.52 & 2.66 & 2.67 & 0.66 & 0.66 \\
\hline Standard error & 0.47 & 0.47 & 0.49 & 0.49 & 0.12 & 0.12 \\
\hline Upper $95 \%$ of mean & 41.67 & 41.67 & 39.99 & 40.00 & -1.48 & -1.47 \\
\hline Lower $95 \%$ of mean & 39.74 & 39.75 & 37.97 & 37.97 & -1.97 & -1.97 \\
\hline Sample size & 29 & 29 & 29 & 29 & 29 & 29 \\
\hline One-sample t-test & & & & & 14.15 & 14.06 \\
\hline P-value & & & & & $<0.0001$ & $<0.0001$ \\
\hline
\end{tabular}

Table A-50. Descriptive statistics and tests for significant posttreatment changes based on repeated measurements for Z Angle $\left({ }^{\circ}\right)$.

\begin{tabular}{|c|c|c|c|c|c|c|}
\hline \multirow[b]{2}{*}{ Variable } & \multicolumn{2}{|c|}{ End of Treatment } & \multicolumn{2}{|c|}{ Recall Examination } & \multicolumn{2}{|c|}{ Posttreatment Change } \\
\hline & $\mathrm{T} 1$ & $\mathrm{~T} 2$ & $\mathrm{~T} 1$ & $\mathrm{~T} 2$ & $\mathrm{~T} 1$ & $\mathrm{~T} 2$ \\
\hline Mean & 76.37 & 76.34 & 80.88 & 80.91 & 4.50 & 4.57 \\
\hline Standard deviation & 6.66 & 6.66 & 6.11 & 6.15 & 4.14 & 4.13 \\
\hline Standard error & 1.22 & 1.22 & 1.12 & 1.12 & 0.76 & 0.75 \\
\hline Upper $95 \%$ of mean & 78.86 & 78.83 & 83.16 & 83.21 & 6.05 & 6.12 \\
\hline Lower $95 \%$ of mean & 73.88 & 73.85 & 78.59 & 78.62 & 2.96 & 3.03 \\
\hline Sample size & 30 & 30 & 30 & 30 & 30 & 30 \\
\hline One-sample t-test & & & & & 5.96 & 6.07 \\
\hline P-value & & & & & $<0.0001$ & $<0.0001$ \\
\hline
\end{tabular}


Table A-51. Descriptive statistics and tests for significant posttreatment changes based on repeated measurements for E Plane-Labrale Superius (mm).

\begin{tabular}{|c|c|c|c|c|c|c|}
\hline \multirow[b]{2}{*}{ Variable } & \multicolumn{2}{|c|}{ End of Treatment } & \multicolumn{2}{|c|}{$\underline{\text { Recall Examination }}$} & \multicolumn{2}{|c|}{ Posttreatment Change } \\
\hline & $\mathrm{T} 1$ & $\mathrm{~T} 2$ & $\mathrm{~T} 1$ & $\mathrm{~T} 2$ & $\mathrm{~T} 1$ & $\mathrm{~T} 2$ \\
\hline Mean & -4.37 & -4.39 & -5.05 & -5.05 & -0.67 & -0.66 \\
\hline Standard deviation & 2.22 & 2.21 & 1.96 & 1.96 & 0.97 & 0.99 \\
\hline Standard error & 0.40 & 0.40 & 0.36 & 0.36 & 0.18 & 0.18 \\
\hline Upper $95 \%$ of mean & -3.55 & -3.56 & -4.32 & -4.32 & -0.31 & -0.29 \\
\hline Lower $95 \%$ of mean & -5.20 & -5.22 & -5.78 & -5.78 & -1.03 & -1.03 \\
\hline Sample size & 30 & 30 & 30 & 30 & 30 & 30 \\
\hline One-sample t-test & & & & & -3.82 & -3.67 \\
\hline P-value & & & & & 0.0007 & 0.001 \\
\hline
\end{tabular}

Table A-52. Descriptive statistics and tests for significant posttreatment changes based on repeated measurements for E Plane-Labrale Inferius ( $\mathrm{mm}$ ).

\begin{tabular}{|c|c|c|c|c|c|c|}
\hline \multirow[b]{2}{*}{ Variable } & \multicolumn{2}{|c|}{ End of Treatment } & \multicolumn{2}{|c|}{$\underline{\text { Recall Examination }}$} & \multicolumn{2}{|c|}{$\underline{\text { Posttreatment Change }}$} \\
\hline & $\mathrm{T} 1$ & $\mathrm{~T} 2$ & $\mathrm{~T} 1$ & $\overline{\mathrm{T} 2}$ & $\mathrm{~T} 1$ & $\mathrm{~T} 2$ \\
\hline Mean & -1.72 & -1.64 & -2.91 & -2.94 & -1.19 & -1.30 \\
\hline Standard deviation & 1.78 & 1.86 & 1.69 & 1.69 & 1.17 & 1.40 \\
\hline Standard error & 0.32 & 0.34 & 0.31 & 0.31 & 0.21 & 0.26 \\
\hline Upper $95 \%$ of mean & -1.06 & -0.95 & -2.28 & -2.31 & -0.75 & -0.77 \\
\hline Lower $95 \%$ of mean & -2.39 & -2.33 & -3.55 & -3.57 & -1.63 & -1.82 \\
\hline Sample size & 30 & 30 & 30 & 30 & 30 & 30 \\
\hline One-sample t-test & & & & & -5.57 & -5.07 \\
\hline P-value & & & & & $<0.0001$ & $<0.0001$ \\
\hline
\end{tabular}


Table A-53. Descriptive statistics and tests for significant posttreatment changes based on repeated measurements for Nasolabial Angle (Labrale Superius-Subnasale-Pronasale).

\begin{tabular}{|c|c|c|c|c|c|c|}
\hline \multirow[b]{2}{*}{ Variable } & \multicolumn{2}{|c|}{ End of Treatment } & \multicolumn{2}{|c|}{ Recall Examination } & \multicolumn{2}{|c|}{ Posttreatment Change } \\
\hline & $\mathrm{T} 1$ & $\mathrm{~T} 2$ & $\mathrm{~T} 1$ & $\mathrm{~T} 2$ & $\mathrm{~T} 1$ & $\mathrm{~T} 2$ \\
\hline Mean & 107.62 & 107.61 & 107.93 & 107.90 & 0.31 & 0.29 \\
\hline Standard deviation & 10.31 & 10.31 & 9.66 & 9.65 & 7.56 & 7.59 \\
\hline Standard error & 1.88 & 1.88 & 1.76 & 1.76 & 1.38 & 1.39 \\
\hline Upper $95 \%$ of mean & 111.47 & 111.46 & 111.54 & 111.50 & 3.13 & 3.12 \\
\hline Lower $95 \%$ of mean & 103.77 & 103.76 & 104.32 & 104.30 & -2.51 & -2.54 \\
\hline Sample size & 30 & 30 & 30 & 30 & 30 & 30 \\
\hline One-sample t-test & & & & & 0.22 & 0.21 \\
\hline P-value & & & & & 0.824 & 0.8356 \\
\hline
\end{tabular}

Table A-54. Descriptive statistics and tests for significant posttreatment changes based on repeated measurements for Mentolabial Angle (Labrale Inferius-Soft Tissue B Point-Soft Tissue Pogonion).

\begin{tabular}{|c|c|c|c|c|c|c|}
\hline \multirow[b]{2}{*}{ Variable } & \multicolumn{2}{|c|}{ End of Treatment } & \multicolumn{2}{|c|}{ Recall Examination } & \multicolumn{2}{|c|}{ Posttreatment Change } \\
\hline & $\mathrm{T} 1$ & $\mathrm{~T} 2$ & $\mathrm{~T} 1$ & $\mathrm{~T} 2$ & $\mathrm{~T} 1$ & $\mathrm{~T} 2$ \\
\hline Mean & 5.00 & 5.02 & 3.29 & 3.31 & -1.71 & -1.71 \\
\hline Standard deviation & 2.10 & 2.11 & 2.25 & 2.23 & 1.58 & 1.57 \\
\hline Standard error & 0.38 & 0.39 & 0.41 & 0.41 & 0.29 & 0.29 \\
\hline Upper $95 \%$ of mean & 5.78 & 5.81 & 4.13 & 4.14 & -1.12 & -1.13 \\
\hline Lower $95 \%$ of mean & 4.22 & 4.23 & 2.45 & 2.48 & -2.30 & -2.30 \\
\hline Sample size & 30 & 30 & 30 & 30 & 30 & 30 \\
\hline One-sample t-test & & & & & -5.93 & -5.96 \\
\hline P-value & & & & & $<0.0001$ & $<0.0001$ \\
\hline
\end{tabular}


Table A-55. Descriptive statistics and tests for significant posttreatment changes based on repeated measurements for Subnasale-Labrale Superius (mm).

\begin{tabular}{|c|c|c|c|c|c|c|}
\hline \multirow[b]{2}{*}{ Variable } & \multicolumn{2}{|c|}{ End of Treatment } & \multicolumn{2}{|c|}{ Recall Examination } & \multicolumn{2}{|c|}{ Posttreatment Change } \\
\hline & $\mathrm{T} 1$ & $\mathrm{~T} 2$ & $\mathrm{~T} 1$ & $\mathrm{~T} 2$ & $\mathrm{~T} 1$ & $\mathrm{~T} 2$ \\
\hline Mean & 18.64 & 18.63 & 20.32 & 20.34 & 1.69 & 1.71 \\
\hline Standard deviation & 2.43 & 2.43 & 2.55 & 2.53 & 1.44 & 1.43 \\
\hline Standard error & 0.44 & 0.44 & 0.46 & 0.46 & 0.26 & 0.26 \\
\hline Upper $95 \%$ of mean & 19.54 & 19.54 & 21.27 & 21.29 & 2.22 & 2.25 \\
\hline Lower $95 \%$ of mean & 17.73 & 17.72 & 19.37 & 19.40 & 1.15 & 1.18 \\
\hline Sample size & 30 & 30 & 30 & 30 & 30 & 30 \\
\hline One-sample t-test & & & & & 6.42 & 6.57 \\
\hline P-value & & & & & $<0.0001$ & $<0.0001$ \\
\hline
\end{tabular}

Table A-56. Descriptive statistics and tests for significant posttreatment changes based on repeated measurements for Subnasale-Labrale Inferius (mm).

\begin{tabular}{|c|c|c|c|c|c|c|}
\hline \multirow[b]{2}{*}{ Variable } & \multicolumn{2}{|c|}{ End of Treatment } & \multicolumn{2}{|c|}{ Recall Examination } & \multicolumn{2}{|c|}{ Posttreatment Change } \\
\hline & $\mathrm{T} 1$ & $\mathrm{~T} 2$ & $\mathrm{~T} 1$ & $\mathrm{~T} 2$ & $\mathrm{~T} 1$ & $\mathrm{~T} 2$ \\
\hline Mean & 25.55 & 25.54 & 27.80 & 27.79 & 2.25 & 2.25 \\
\hline Standard deviation & 3.70 & 3.69 & 3.56 & 3.57 & 2.21 & 2.20 \\
\hline Standard error & 0.67 & 0.67 & 0.65 & 0.65 & 0.40 & 0.40 \\
\hline Upper $95 \%$ of mean & 26.93 & 26.92 & 29.13 & 29.13 & 3.07 & 3.07 \\
\hline Lower $95 \%$ of mean & 24.17 & 24.17 & 26.47 & 26.46 & 1.42 & 1.43 \\
\hline Sample size & 30 & 30 & 30 & 30 & 30 & 30 \\
\hline One-sample t-test & & & & & 5.58 & 5.61 \\
\hline P-value & & & & & $<0.0001$ & $<0.0001$ \\
\hline
\end{tabular}


Table A-57. Descriptive statistics and tests for significant posttreatment changes based on repeated measurements for Nasion Perpendicular-Pronasale ( $\mathrm{mm})$.

\begin{tabular}{|c|c|c|c|c|c|c|}
\hline \multirow[b]{2}{*}{ Variable } & \multicolumn{2}{|c|}{ End of Treatment } & \multicolumn{2}{|c|}{ Recall Examination } & \multicolumn{2}{|c|}{ Posttreatment Change } \\
\hline & $\mathrm{T} 1$ & $\mathrm{~T} 2$ & $\mathrm{~T} 1$ & $\mathrm{~T} 2$ & $\mathrm{~T} 1$ & $\mathrm{~T} 2$ \\
\hline Mean & 30.59 & 30.61 & 32.48 & 32.50 & 1.89 & 1.89 \\
\hline Standard deviation & 3.14 & 3.13 & 3.24 & 3.24 & 0.64 & 0.60 \\
\hline Standard error & 0.57 & 0.57 & 0.59 & 0.59 & 0.12 & 0.11 \\
\hline Upper $95 \%$ of mean & 31.77 & 31.78 & 33.69 & 33.71 & 2.12 & 2.11 \\
\hline Lower $95 \%$ of mean & 29.42 & 29.44 & 31.27 & 31.29 & 1.65 & 1.66 \\
\hline Sample size & 30 & 30 & 30 & 30 & 30 & 30 \\
\hline One-sample t-test & & & & & 16.27 & 17.16 \\
\hline P-value & & & & & $<0.0001$ & $<0.0001$ \\
\hline
\end{tabular}

Table A-58. Descriptive statistics and tests for significant posttreatment changes based on repeated measurements for W Point-Soft Tissue Pogonion (mm).

\begin{tabular}{|c|c|c|c|c|c|c|}
\hline \multirow[b]{2}{*}{ Variable } & \multicolumn{2}{|c|}{ End of Treatment } & \multicolumn{2}{|c|}{$\underline{\text { Recall Examination }}$} & \multicolumn{2}{|c|}{$\underline{\text { Posttreatment Change }}$} \\
\hline & $\mathrm{T} 1$ & $\mathrm{~T} 2$ & $\mathrm{~T} 1$ & $\overline{\mathrm{T} 2}$ & $\mathrm{~T} 1$ & $\mathrm{~T} 2$ \\
\hline Mean & 24.62 & 24.62 & 26.15 & 26.15 & 1.53 & 1.53 \\
\hline Standard deviation & 2.72 & 2.71 & 2.75 & 2.73 & 0.60 & 0.63 \\
\hline Standard error & 0.50 & 0.49 & 0.50 & 0.50 & 0.11 & 0.12 \\
\hline Upper $95 \%$ of mean & 25.64 & 25.64 & 27.18 & 27.17 & 1.76 & 1.77 \\
\hline Lower $95 \%$ of mean & 23.60 & 23.61 & 25.13 & 25.13 & 1.31 & 1.29 \\
\hline Sample size & 30 & 30 & 30 & 30 & 30 & 30 \\
\hline One-sample t-test & & & & & 13.98 & 13.26 \\
\hline P-value & & & & & $<0.0001$ & $<0.0001$ \\
\hline
\end{tabular}


APPENDIX B.

RESULTS OF MIXED-MODEL ANOVA TESTING FOR POSTTREATMENT CHANGES 
Table B-1. Results of mixed-model ANOVA testing for posttreatment changes.

\begin{tabular}{|c|c|c|c|c|c|c|c|c|c|c|}
\hline \multirow[b]{2}{*}{ Variable } & \multicolumn{2}{|c|}{ End of TX } & \multicolumn{2}{|c|}{ Recall Exam } & \multicolumn{2}{|c|}{ Growth } & \multicolumn{2}{|c|}{ Repetitions } & \multicolumn{2}{|c|}{ Interaction } \\
\hline & $\mathrm{T} 1$ & $\overline{\mathrm{T}} 2$ & $\overline{\mathrm{T} 1}$ & $\mathrm{~T} 2$ & F-Ratio & $\overline{\text { P-Value }}$ & F Ratio & P-Value & F Ratio & P-Value \\
\hline Se-Na & 71.90 & 71.88 & 74.63 & 74.62 & 19.25 & $<0.0001$ & 0.72 & 0.4009 & 0.42 & 0.5190 \\
\hline Se-Ba & 41.43 & 41.40 & 43.64 & 43.63 & 9.58 & 0.0030 & 2.36 & 0.1302 & 1.78 & 0.1878 \\
\hline $\mathrm{Na}-\mathrm{Ba}$ & 104.23 & 104.21 & 109.74 & 109.73 & 28.98 & $<0.0001$ & 0.73 & 0.3958 & 0.01 & 0.9036 \\
\hline Na-Se-Ba & 133.52 & 133.55 & 133.54 & 133.48 & 0.00 & 0.9798 & 0.35 & 0.5587 & 1.62 & 0.2076 \\
\hline Na-ANS & 49.88 & 49.89 & 53.01 & 53.01 & 22.24 & $<0.0001$ & 0.05 & 0.8306 & 0.16 & 0.6922 \\
\hline Se-PNS & 52.88 & 52.90 & 55.62 & 55.60 & 14.71 & 0.0003 & 0.05 & 0.8272 & 3.53 & 0.0654 \\
\hline $\mathrm{Cd}-\mathrm{A}$ & 88.99 & 88.99 & 92.54 & 92.52 & 8.32 & 0.0055 & 0.31 & 0.5815 & 1.00 & 0.3216 \\
\hline Na-PP & 49.88 & 49.86 & 53.01 & 53.02 & 22.40 & $<0.0001$ & 0.37 & 0.5477 & 5.34 & 0.0244 \\
\hline Me-PP & 64.39 & 64.36 & 68.73 & 68.73 & 7.59 & 0.0078 & 2.58 & 0.1136 & 5.13 & 0.0272 \\
\hline $\mathrm{Na}-\mathrm{Me}$ & 112.86 & 112.88 & 120.05 & 120.04 & 15.91 & 0.0002 & 0.08 & 0.7794 & 1.88 & 0.1759 \\
\hline Se-Go & 75.64 & 75.64 & 79.79 & 79.76 & 12.83 & 0.0007 & 1.12 & 0.2946 & 2.39 & 0.1279 \\
\hline $\mathrm{PFH} / \mathrm{AFH}$ & 0.67 & 0.67 & 0.67 & 0.67 & 0.16 & 0.6922 & 0.82 & 0.3702 & 0.00 & 0.9693 \\
\hline SNA & 79.92 & 79.92 & 80.36 & 80.36 & 0.21 & 0.6482 & 0.00 & 1.0000 & 0.00 & 1.0000 \\
\hline NaPerp-A & -0.54 & -0.56 & 0.87 & 0.88 & 5.76 & 0.0196 & 0.65 & 0.4218 & 1.47 & 0.2299 \\
\hline $\mathrm{PtV}-\mathrm{A}$ & 45.00 & 44.96 & 47.70 & 47.71 & 16.87 & 0.0001 & 3.29 & 0.0750 & 4.48 & 0.0387 \\
\hline Se-A & 81.91 & 81.91 & 86.29 & 86.27 & 24.83 & $<0.0001$ & 0.45 & 0.5062 & 1.16 & 0.2858 \\
\hline
\end{tabular}


Table B-1. Continued.

\begin{tabular}{|c|c|c|c|c|c|c|c|c|c|c|}
\hline \multirow[b]{2}{*}{ Variable } & \multicolumn{2}{|c|}{ End of TX } & \multicolumn{2}{|c|}{ Recall Exam } & \multicolumn{2}{|c|}{ Growth } & \multicolumn{2}{|c|}{ Repetitions } & \multicolumn{2}{|c|}{ Interaction } \\
\hline & $\overline{\mathrm{T} 1}$ & $\mathrm{~T} 2$ & $\overline{\mathrm{T} 1}$ & $\mathrm{~T} 2$ & F Ratio & $\overline{\text { P-Value }}$ & F Ratio & $\overline{\text { P-Value }}$ & F Ratio & $\overline{\text { P-Value }}$ \\
\hline PP-SN & 7.13 & 7.14 & 7.58 & 7.61 & 0.34 & 0.5596 & 1.67 & 0.2011 & 1.01 & 0.3187 \\
\hline SNB & 76.38 & 76.34 & 77.15 & 77.18 & 1.08 & 0.3034 & 0.02 & 0.8974 & 7.40 & 0.0086 \\
\hline NaPerp-B & -5.82 & -5.84 & -3.12 & -3.14 & 9.81 & 0.0027 & 2.73 & 0.1038 & 0.20 & 0.6539 \\
\hline PtV-B & 44.19 & 44.23 & 46.63 & 46.64 & 10.39 & 0.0021 & 3.71 & 0.0590 & 1.89 & 0.1742 \\
\hline Cd-Gn & 114.25 & 114.22 & 120.83 & 120.83 & 25.38 & $<0.0001$ & 1.41 & 0.2407 & 1.32 & 0.2556 \\
\hline Cd-Go & 60.41 & 60.43 & 65.72 & 65.72 & 17.02 & 0.0001 & 1.80 & 0.1851 & 2.14 & 0.1492 \\
\hline Go-Pg & 76.16 & 76.14 & 80.67 & 80.70 & 13.60 & 0.0005 & 0.01 & 0.9062 & 5.94 & 0.0179 \\
\hline Y-Axis & 57.22 & 57.23 & 56.72 & 56.77 & 0.23 & 0.6368 & 1.52 & 0.2233 & 0.85 & 0.3597 \\
\hline NaPerp-Pg & -3.60 & -3.62 & -1.59 & -1.59 & 3.10 & 0.0835 & 0.49 & 0.4863 & 1.59 & 0.2123 \\
\hline ANB & 3.51 & 3.54 & 3.21 & 3.18 & 0.42 & 0.5186 & 0.04 & 0.8333 & 3.62 & 0.0621 \\
\hline AO-BO & 1.08 & 1.08 & 0.57 & 0.61 & 0.41 & 0.5229 & 3.74 & 0.0579 & 3.74 & 0.0579 \\
\hline FMA & 20.60 & 20.60 & 20.06 & 20.09 & 0.10 & 0.7554 & 0.84 & 0.3639 & 0.84 & 0.3639 \\
\hline Na-A-Pg & 3.47 & 3.46 & 3.94 & 3.97 & 0.16 & 0.6904 & 0.53 & 0.4678 & 1.73 & 0.1935 \\
\hline FH-Na-Pg & 87.66 & 87.74 & 88.98 & 88.99 & 2.73 & 0.1038 & 1.22 & 0.2732 & 0.72 & 0.3983 \\
\hline U1/L1 & 118.68 & 118.61 & 125.24 & 125.26 & 12.42 & 0.0008 & 0.42 & 0.5215 & 1.16 & 0.2868 \\
\hline U1/SN & 107.21 & 107.17 & 104.11 & 104.17 & 3.54 & 0.0650 & 0.15 & 0.7029 & 6.40 & 0.0141 \\
\hline
\end{tabular}


Table B-1. Continued.

\begin{tabular}{|c|c|c|c|c|c|c|c|c|c|c|}
\hline \multirow[b]{2}{*}{ Variable } & \multicolumn{2}{|c|}{ End of TX } & \multicolumn{2}{|c|}{ Recall Exam } & \multicolumn{2}{|c|}{ Growth } & \multicolumn{2}{|c|}{ Repetitions } & \multicolumn{2}{|c|}{ Interaction } \\
\hline & $\mathrm{T} 1$ & $\mathrm{~T} 2$ & $\overline{\mathrm{T} 1}$ & $\mathrm{~T} 2$ & F Ratio & $\overline{\text { P-Value }}$ & F Ratio & P-Value & F Ratio & P-Value \\
\hline $\mathrm{U} 1-\mathrm{NA}{ }^{\circ}$ & 27.29 & 27.22 & 23.74 & 23.70 & 5.15 & 0.0269 & 8.16 & 0.0059 & 0.79 & 0.3790 \\
\hline U1-NA mm & 3.10 & 3.13 & 2.87 & 2.89 & 0.17 & 0.6793 & 5.56 & 0.0217 & 0.22 & 0.6389 \\
\hline L1-NB ${ }^{\circ}$ & 30.67 & 30.64 & 27.79 & 27.80 & 4.61 & 0.0359 & 1.19 & 0.2792 & 1.78 & 0.1871 \\
\hline L1-NB mm & 4.75 & 4.79 & 3.42 & 3.43 & 8.44 & 0.0052 & 7.26 & 0.0092 & 2.84 & 0.0975 \\
\hline IMPA & 103.69 & 103.67 & 99.54 & 99.57 & 4.58 & 0.0365 & 0.01 & 0.9425 & 0.89 & 0.3502 \\
\hline FMIA & 55.39 & 55.40 & 60.00 & 60.03 & 9.89 & 0.0026 & 1.88 & 0.1754 & 0.76 & 0.3863 \\
\hline U1-Apg & 4.53 & 4.57 & 4.55 & 4.57 & 0.00 & 0.9817 & 5.55 & 0.0219 & 1.02 & 0.3168 \\
\hline L1-APg & 1.78 & 1.74 & 0.85 & 0.88 & 4.76 & 0.0332 & 0.67 & 0.4162 & 16.76 & 0.0001 \\
\hline D Occ Pl-FH & 6.29 & 6.30 & 4.41 & 4.47 & 4.44 & 0.0395 & 9.34 & 0.0034 & 2.56 & 0.1148 \\
\hline Overbite & 2.12 & 2.16 & 3.02 & 3.06 & 9.26 & 0.0035 & 6.99 & 0.0105 & 0.00 & 1.0000 \\
\hline Overjet & 2.81 & 2.77 & 3.76 & 3.78 & 26.01 & $<0.0001$ & 0.58 & 0.4499 & 6.69 & 0.0122 \\
\hline L1-W Pt & 9.49 & 9.53 & 9.44 & 9.44 & 0.03 & 0.8749 & 2.64 & 0.1095 & 3.95 & 0.0517 \\
\hline Distal L6-W Pt & 15.65 & 15.67 & 14.72 & 14.72 & 3.24 & 0.0769 & 0.12 & 0.7330 & 0.64 & 0.4270 \\
\hline U1-PtV & 51.27 & 51.32 & 54.23 & 54.26 & 7.62 & 0.0077 & 6.98 & 0.0106 & 0.44 & 0.5116 \\
\hline U6-PtV & 16.39 & 16.40 & 18.75 & 18.76 & 8.27 & 0.0057 & 1.09 & 0.3015 & 0.07 & 0.7953 \\
\hline Distal L6-L1 & 35.19 & 35.18 & 33.95 & 33.93 & 7.39 & 0.0087 & 2.11 & 0.1518 & 0.09 & 0.7655 \\
\hline
\end{tabular}


Table B-1. Continued.

\begin{tabular}{lccccccccccc}
\hline & \multicolumn{2}{c}{ End of TX } & \multicolumn{2}{c}{ Recall Exam } & & \multicolumn{2}{c}{ Growth } & \multicolumn{2}{c}{ Repetitions } & \multicolumn{2}{c}{ Interaction } \\
Variable & T1 & T2 & & T1 & T2 & F Ratio & P-Value & F Ratio & P-Value & F Ratio & P-Value \\
\hline Distal U6-U1 & 40.71 & 40.71 & 38.98 & 38.99 & 6.39 & 0.0143 & 0.26 & 0.6096 & 0.09 & 0.7602 \\
Z Angle & 76.37 & 76.34 & 80.88 & 80.91 & 7.54 & 0.0080 & 0.01 & 0.9226 & 4.19 & 0.0451 \\
E Plane-Ls & -4.37 & -4.39 & -5.05 & -5.05 & 1.54 & 0.2204 & 1.08 & 0.3040 & 0.20 & 0.6583 \\
E Plane-Li & -1.72 & 1.73 & -2.91 & -2.90 & 6.99 & 0.0105 & 0.09 & 0.7671 & 0.35 & 0.5541 \\
Nasolabial Angle & 107.62 & 107.61 & 107.93 & 107.90 & 0.01 & 0.9078 & 0.45 & 0.5061 & 0.11 & 0.7392 \\
Li-B'-Pg' & 125.35 & 125.38 & 127.77 & 127.76 & 1.67 & 0.2011 & 0.04 & 0.8499 & 0.23 & 0.6364 \\
Sn-Ls & 18.64 & 18.63 & 20.32 & 20.34 & 7.03 & 0.0103 & 0.26 & 0.6096 & 1.05 & 0.3087 \\
Sn-Li & 25.55 & 25.54 & 27.80 & 27.79 & 5.76 & 0.0197 & 0.41 & 0.5232 & 0.02 & 0.8982 \\
NaPerp-Pr & 30.59 & 30.61 & 32.48 & 32.50 & 5.26 & 0.0255 & 2.18 & 0.1452 & 0.00 & 1.0000 \\
W Pt-Pg' & 24.62 & 24.62 & 26.15 & 26.15 & 4.73 & 0.0337 & 0.03 & 0.8721 & 0.03 & 0.8721 \\
\hline
\end{tabular}




\section{APPENDIX C.}

GRAPHICAL REPRESENTATIONS OF THE DISTRIBUTIONS OF POSTTREATMENT CHANGE FOR EACH CEPHALOMETRIC VARIABLE 


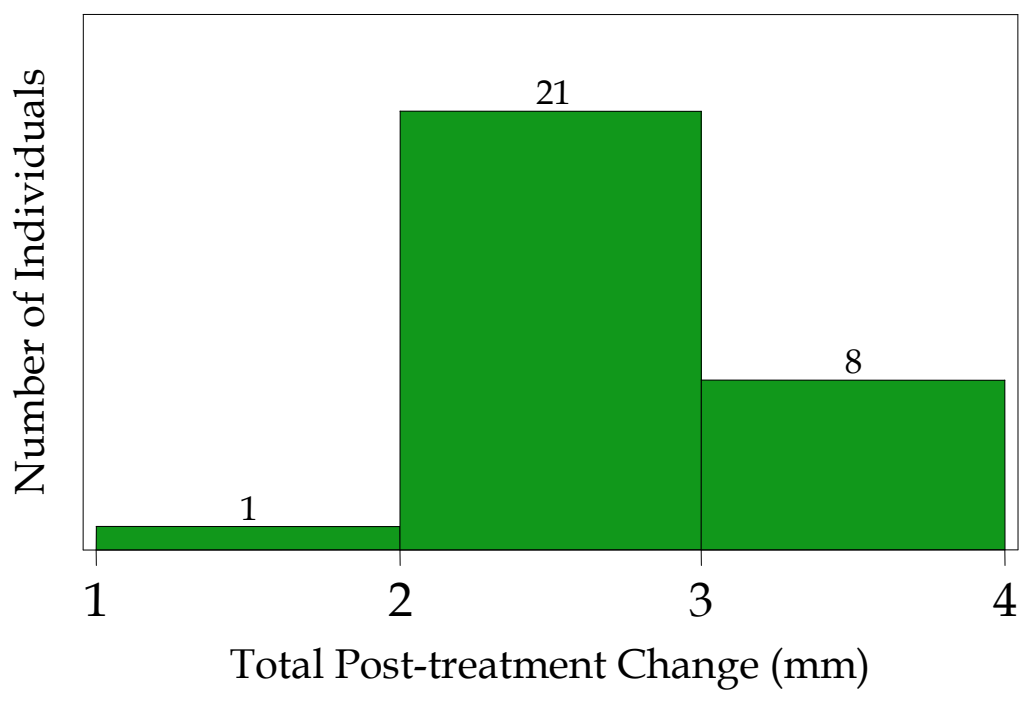

Figure C-1. Plot of the distribution of posttreatment changes in Sella-Nasion distance among the 30 women in the sample. Numbers atop each bar are the numbers of cases in that interval.

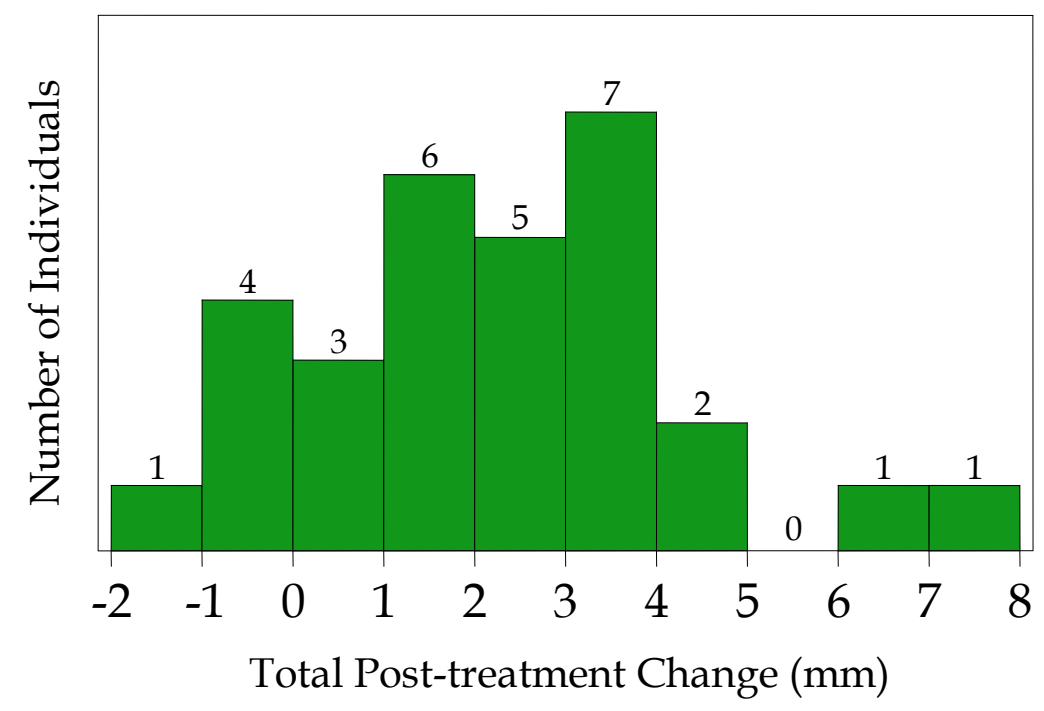

Figure C-2. Plot of the distribution of posttreatment changes in Sella-Basion distance among the 30 women in the sample. Numbers atop each bar are the numbers of cases in that interval. 


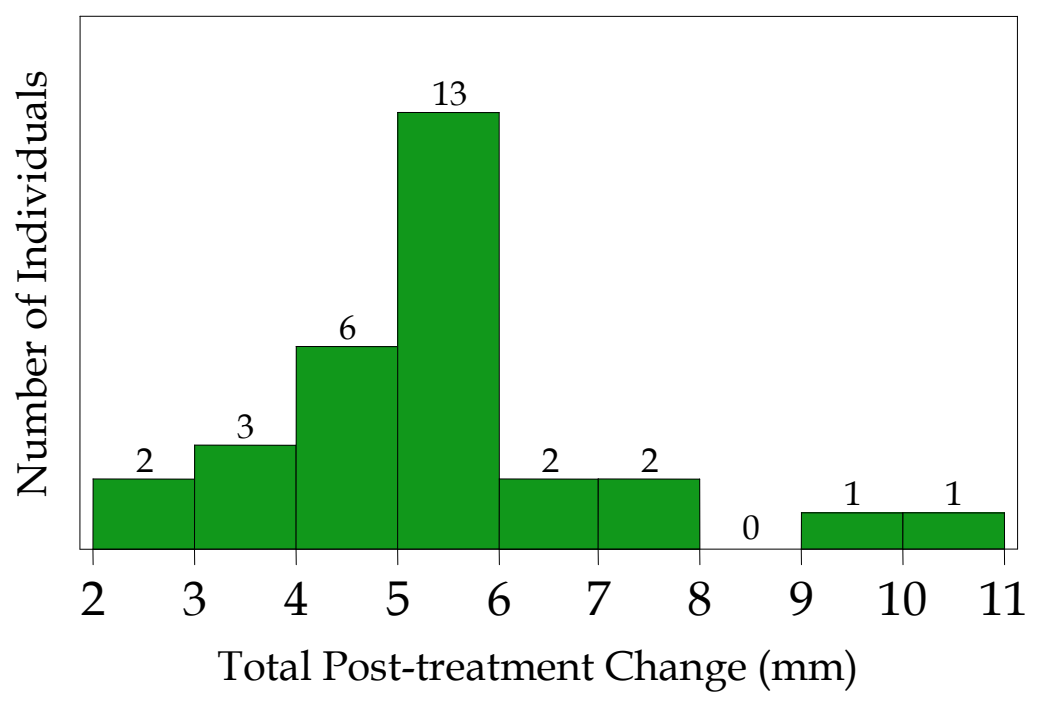

Figure C-3. Plot of the distribution of posttreatment changes in Nasion-Basion distance among the 30 women in the sample. Numbers atop each bar are the numbers of cases in that interval.

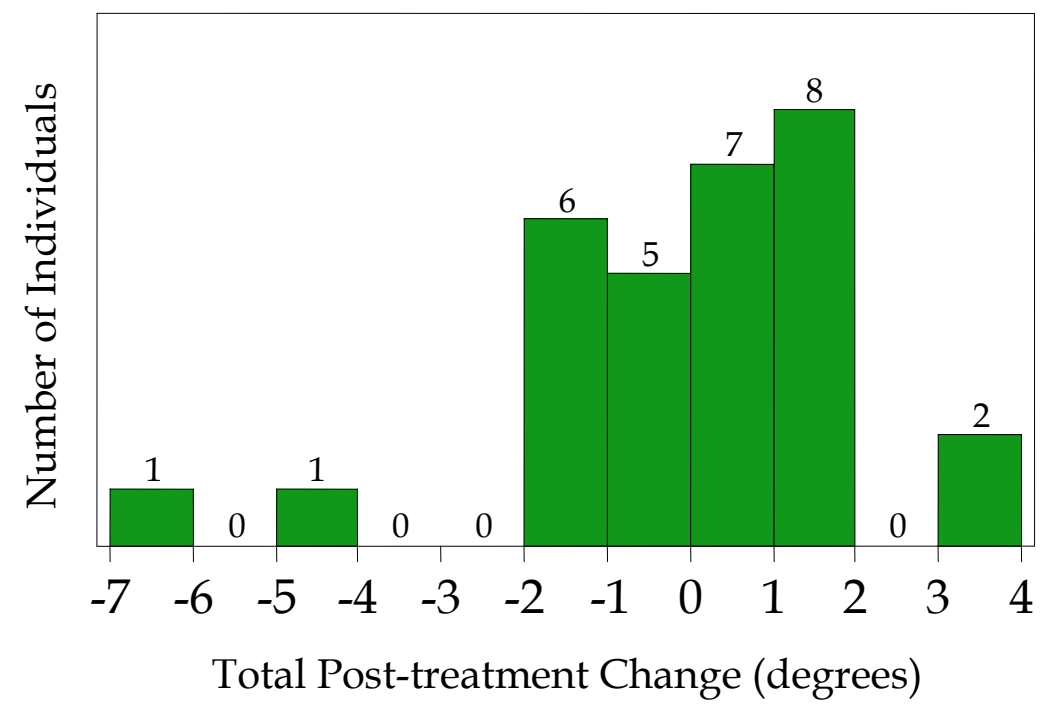

Figure C-4. Plot of the distribution of posttreatment changes in Nasion-SellaBasion angle among the 30 women in the sample. Numbers atop each bar are the numbers of cases in that interval. 


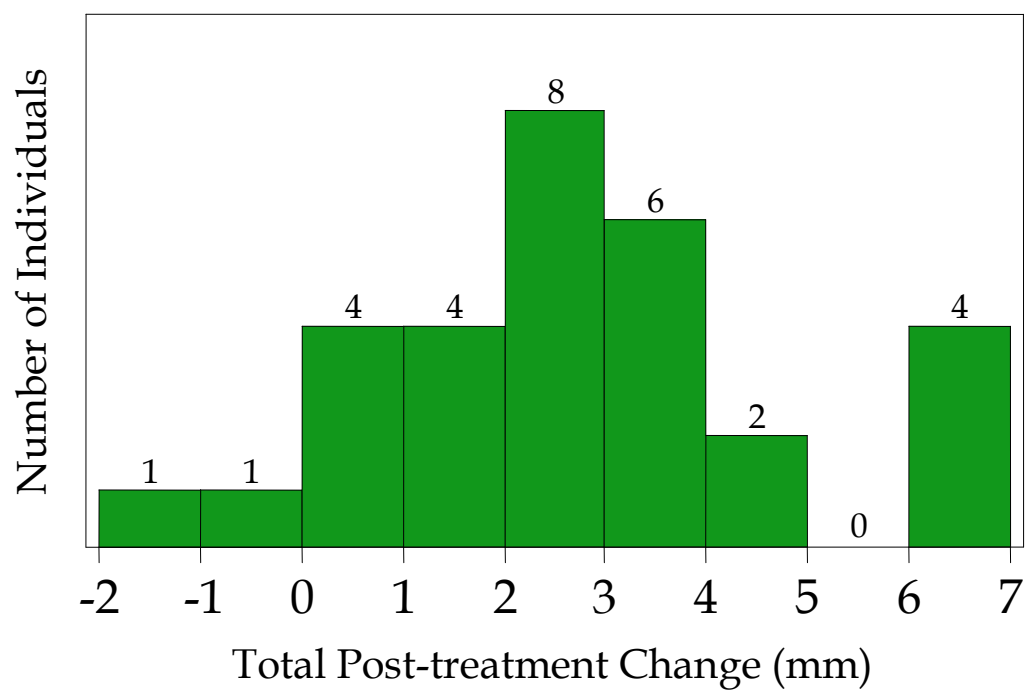

Figure C-5. Plot of the distribution of posttreatment changes in Sella-Posterior Nasal Spine distance among the 30 women in the sample. Numbers atop each bar are the numbers of cases in that interval.

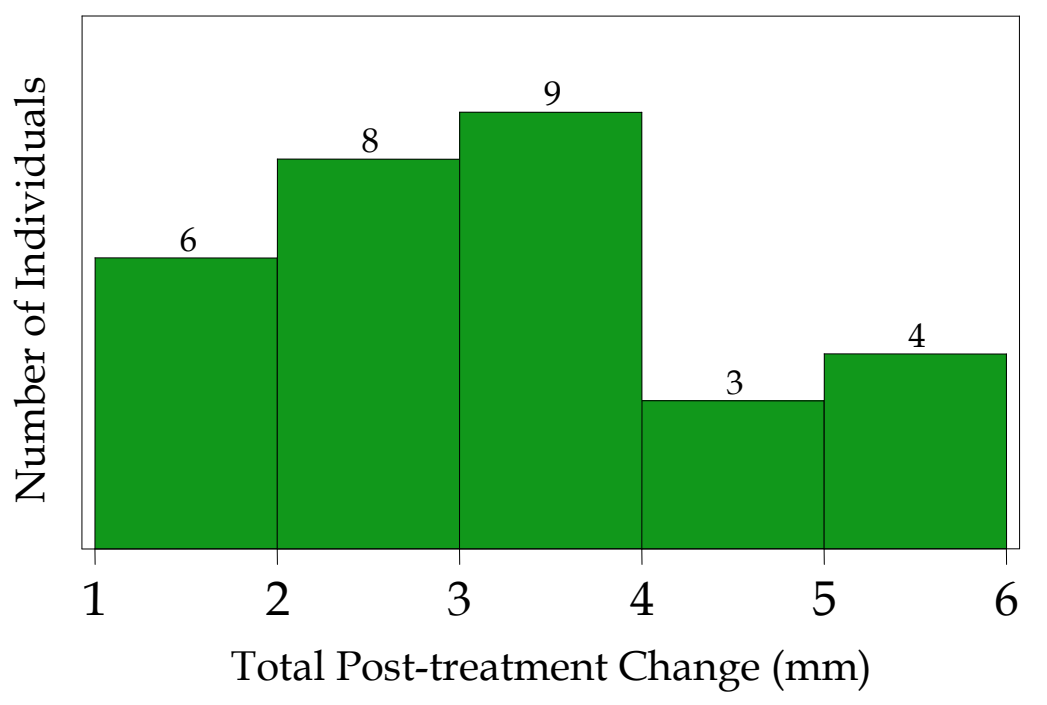

Figure C-6. Plot of the distribution of posttreatment changes in Nasion-Palatal Plane distance among the 30 women in the sample. Numbers atop each bar are the numbers of cases in that interval. 


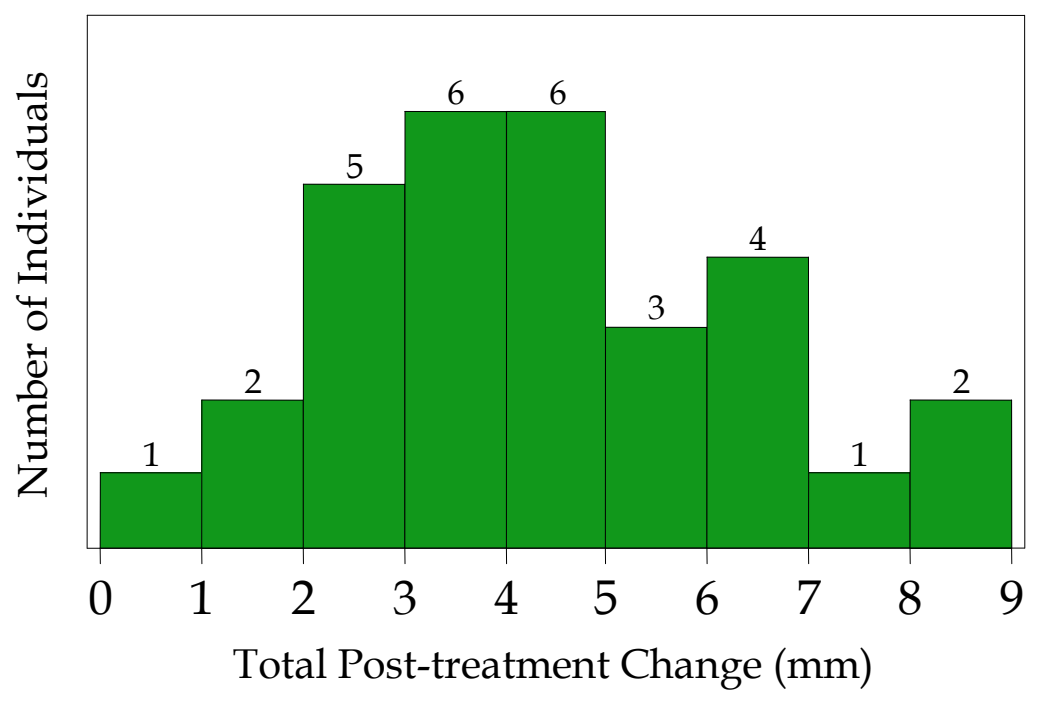

Figure C-7. Plot of the distribution of posttreatment changes in Menton-Palatal Plane distance among the 30 women in the sample. Numbers atop each bar are the numbers of cases in that interval.

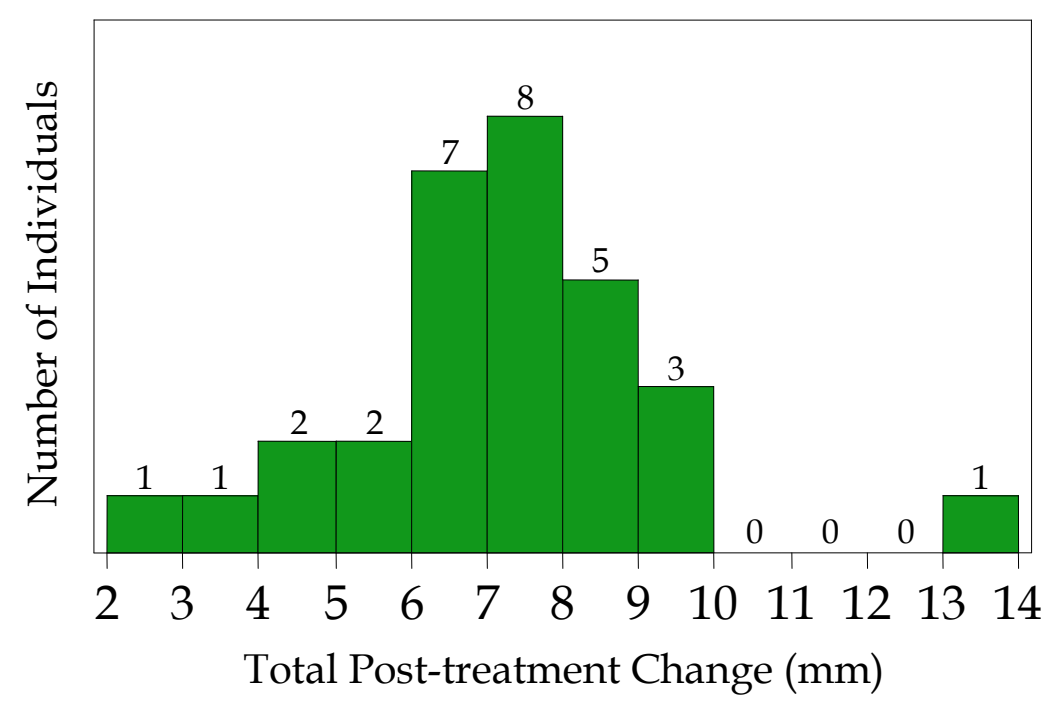

Figure C-8. Plot of the distribution of posttreatment changes in Nasion-Menton distance among the 30 women in the sample. Numbers atop each bar are the numbers of cases in that interval. 


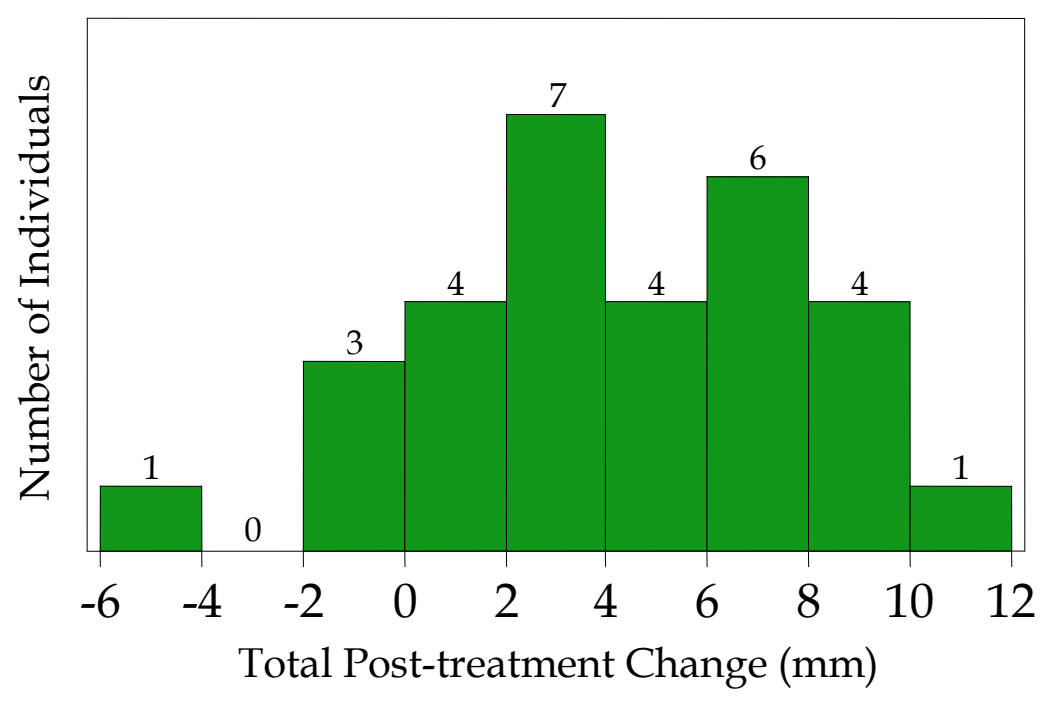

Figure C-9. Plot of the distribution of posttreatment changes in Sella-Gonion distance among the 30 women in the sample. Numbers atop each bar are the numbers of cases in that interval.

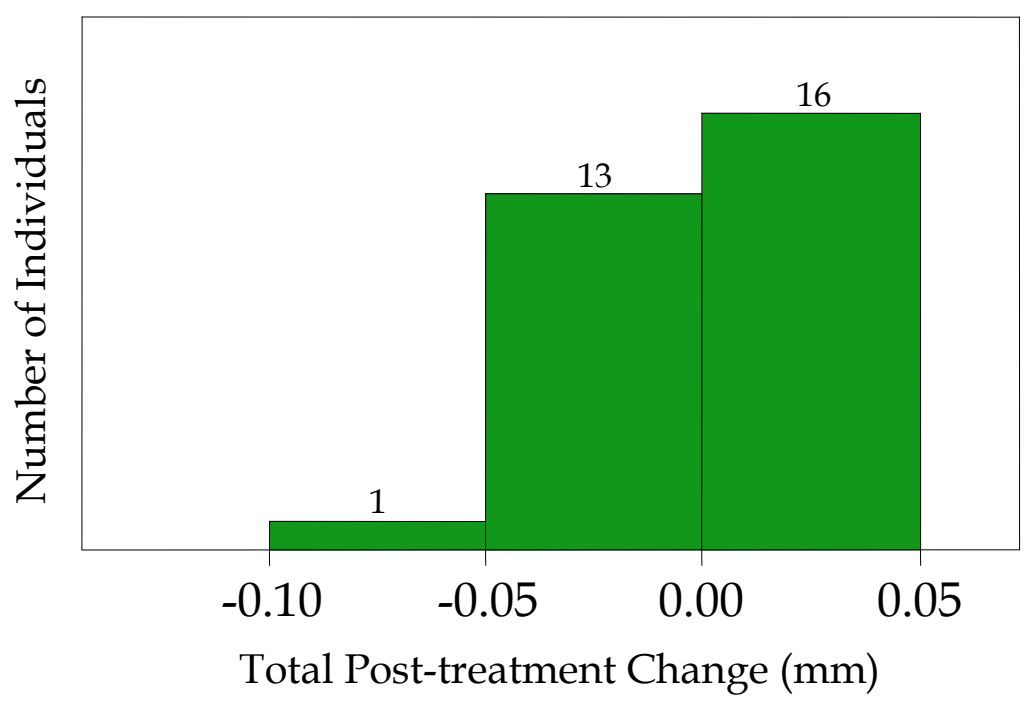

Figure C-10. Plot of the distribution of posttreatment changes in Posterior Facial Height/Anterior Facial Height ratio. among the 30 women in the sample. Numbers atop each bar are the numbers of cases in that interval. 


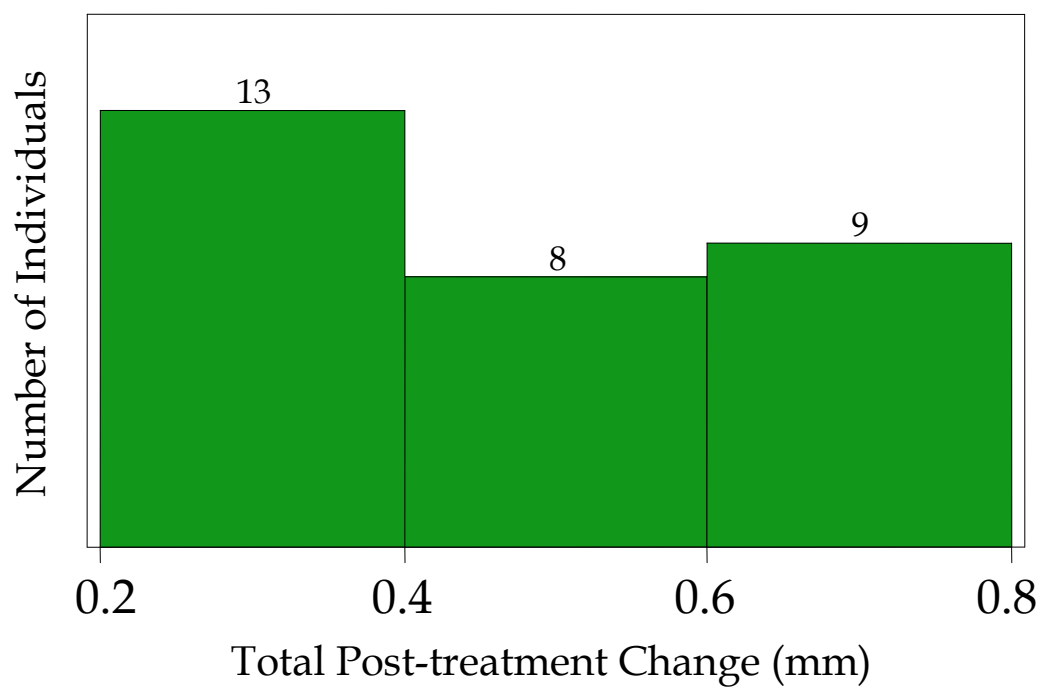

Figure C-11. Plot of the distribution of posttreatment changes in Sella-Nasion-A Point angle among the 30 women in the sample. Numbers atop each bar are the numbers of cases in that interval.

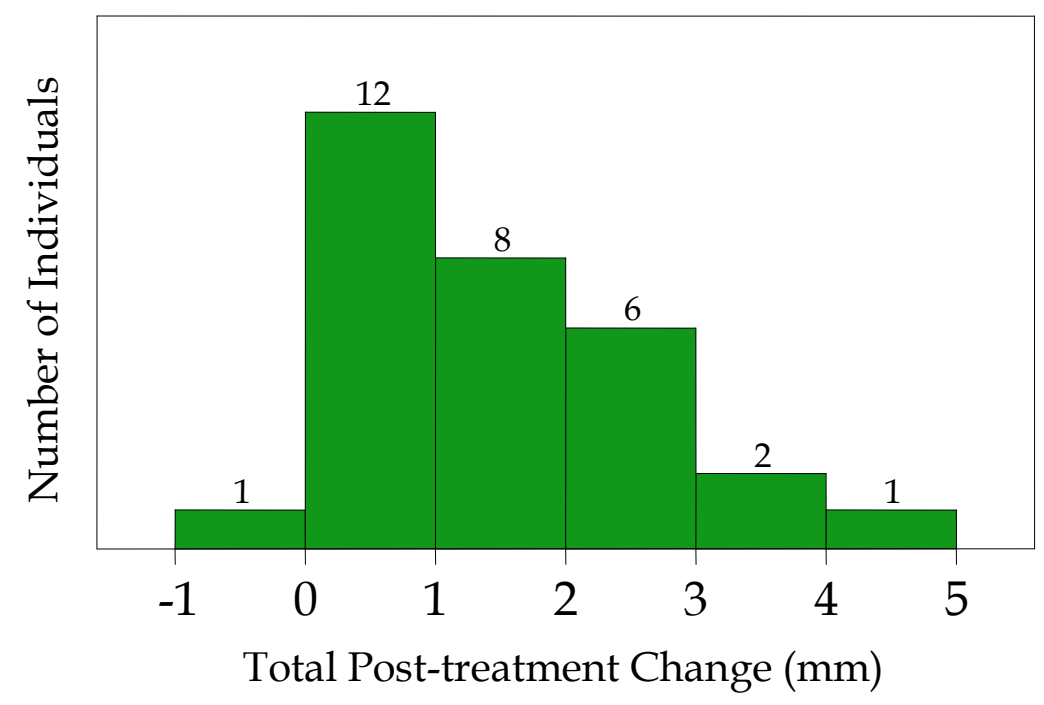

Figure C-12. Plot of the distribution of posttreatment changes in Nasion Perpendicular-A Point distance among the 30 women in the sample. Numbers atop each bar are the numbers of cases in that interval. 


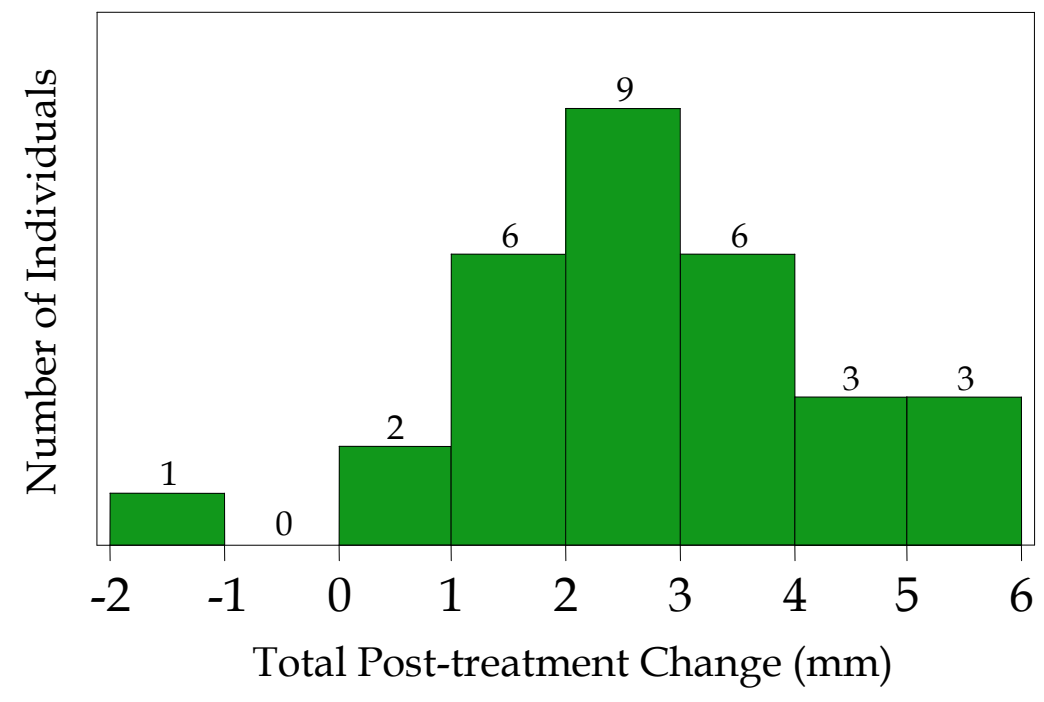

Figure C-13. Plot of the distribution of posttreatment changes in Pterygoid Vertical-A Point distance among the 30 women in the sample. Numbers atop each bar are the numbers of cases in that interval.

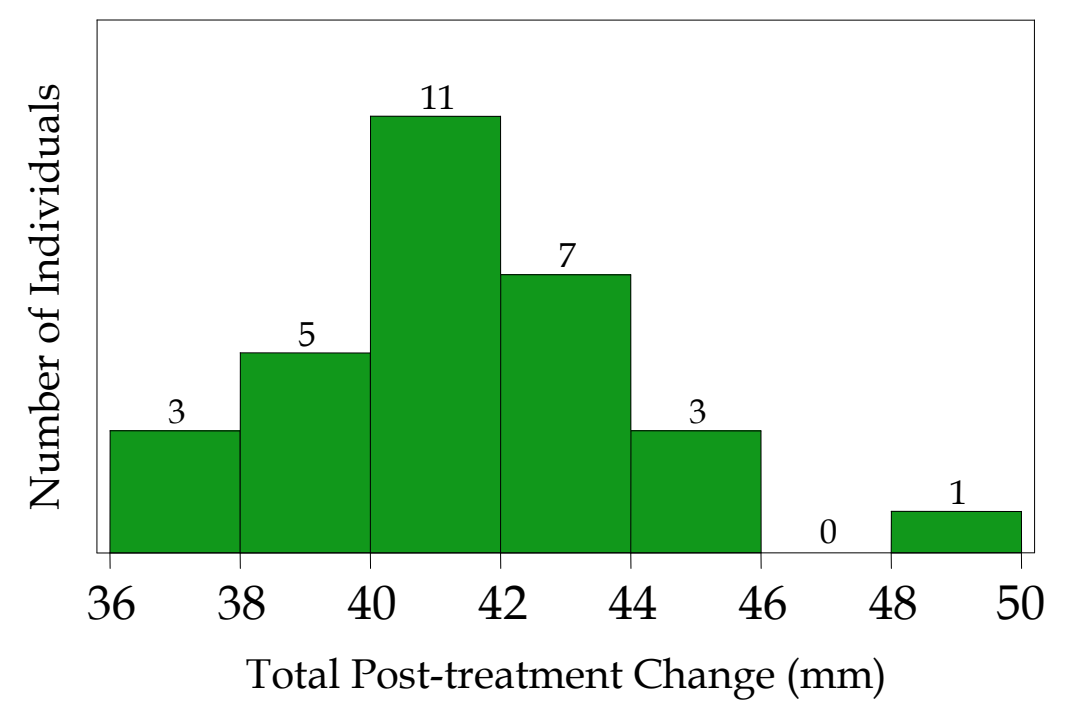

Figure C-14. Plot of the distribution of posttreatment changes in Sella-A Point distance among the 30 women in the sample. Numbers atop each bar are the numbers of cases in that interval. 


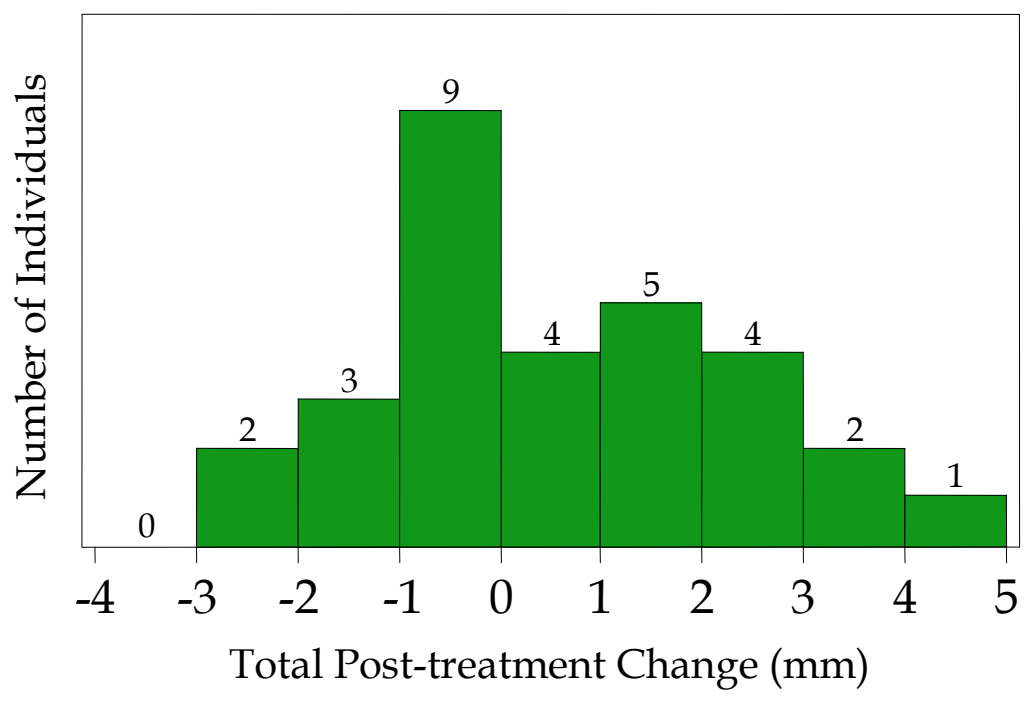

Figure C-15. Plot of the distribution of posttreatment changes in Palatal Plane to Sella-Nasion distance among the 30 women in the sample. Numbers atop each bar are the numbers of cases in that interval.

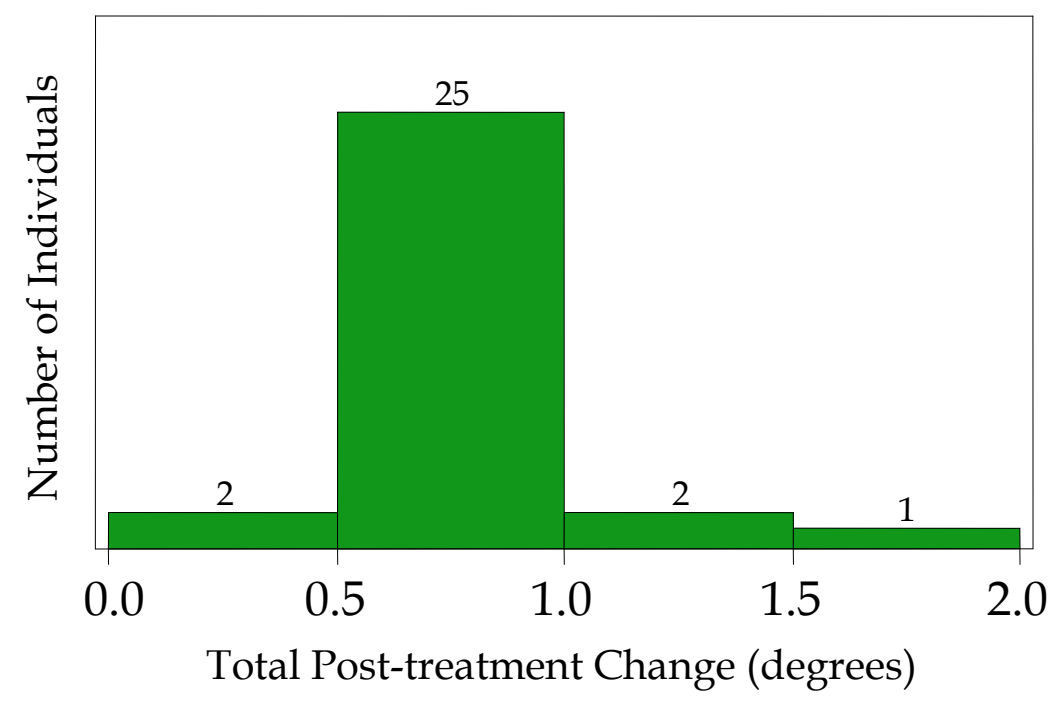

Figure C-16. Plot of the distribution of posttreatment changes in Sella-Nasion-B Point angle among the 30 women in the sample. Numbers atop each bar are the numbers of cases in that interval. 


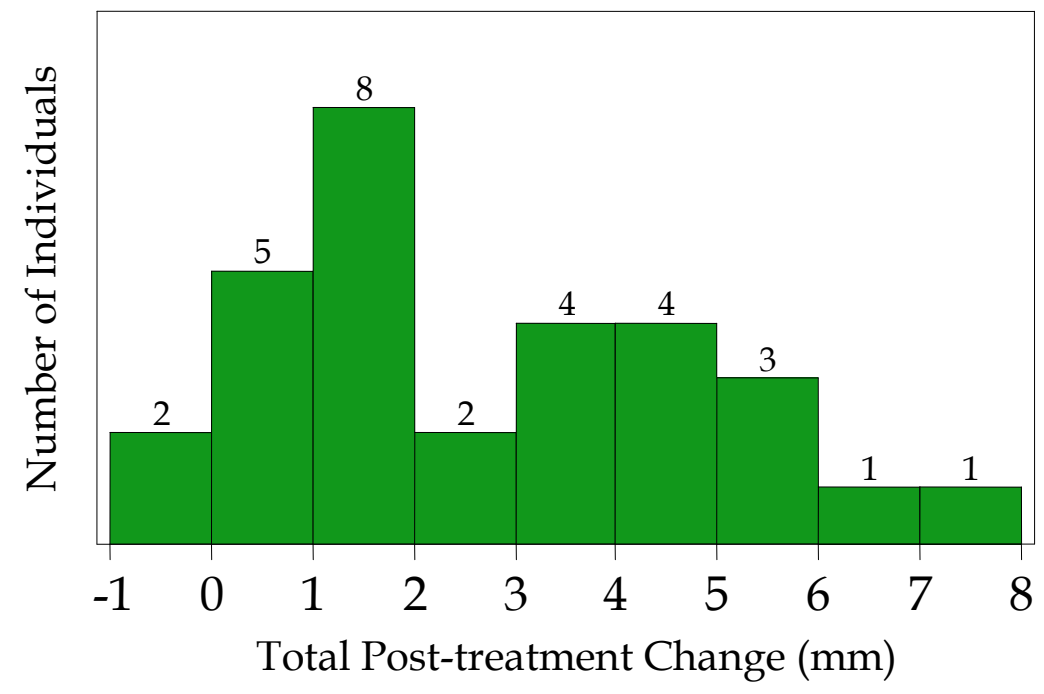

Figure C-17. Plot of the distribution of posttreatment changes in Nasion Perpendicular-B point distance among the 30 women in the sample. Numbers atop each bar are the numbers of cases in that interval.

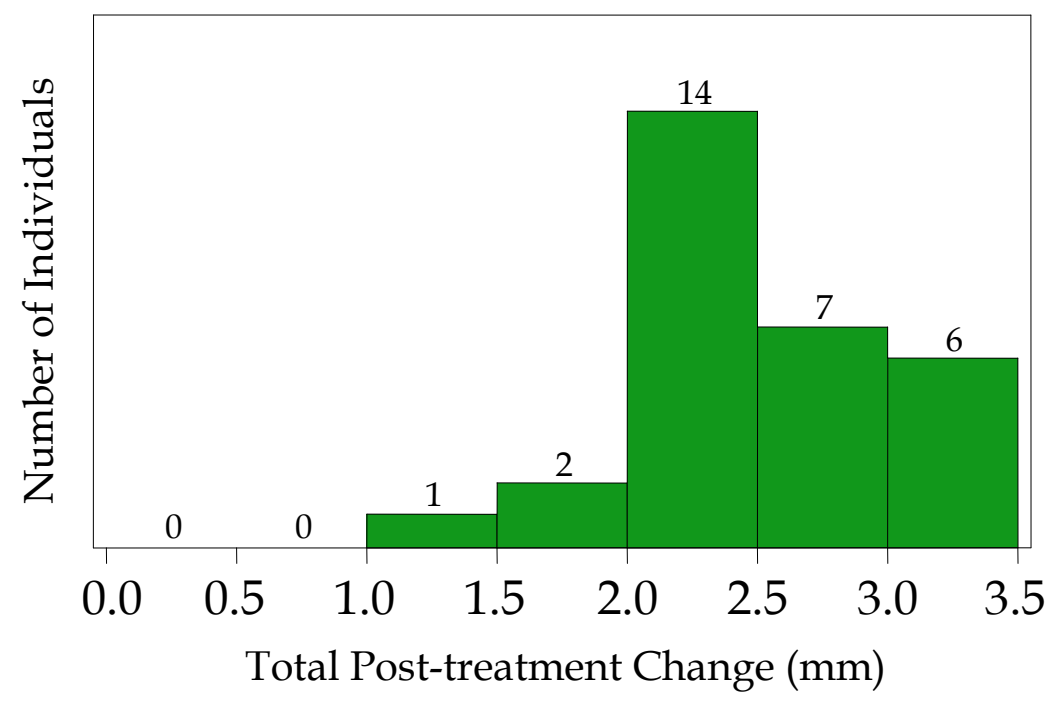

Figure C-18. Plot of the distribution of posttreatment changes in Pterygoid Vertical-B Point distance among the 30 women in the sample. Numbers atop each bar are the numbers of cases in that interval. 


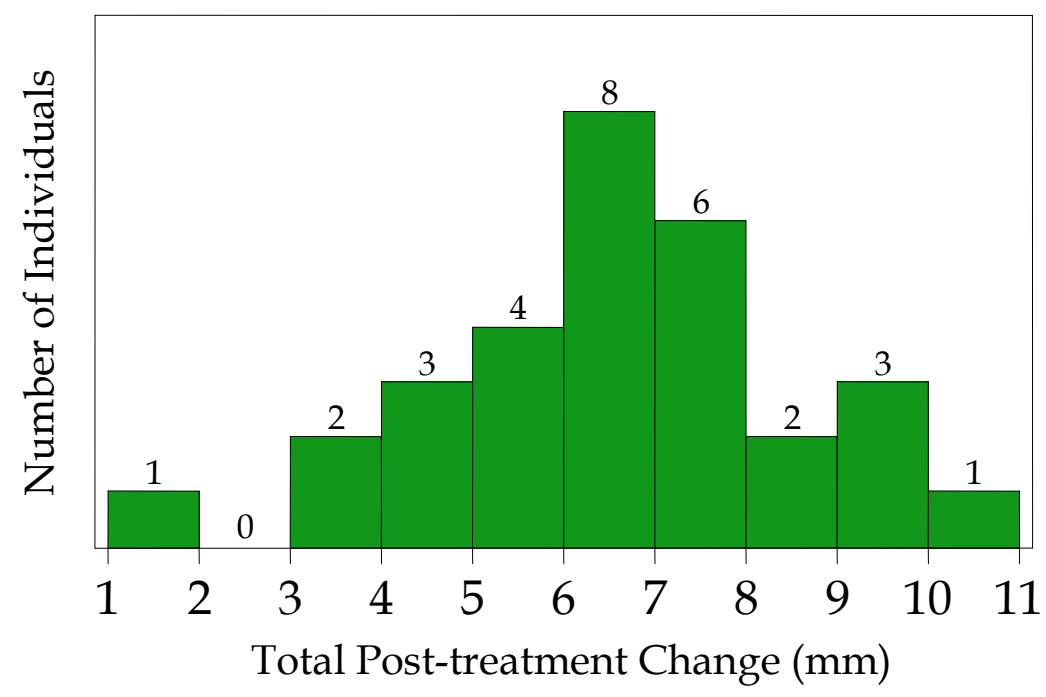

Figure C-19. Plot of the distribution of posttreatment changes in CondylionGnathion distance among the 30 women in the sample. Numbers atop each bar are the numbers of cases in that interval.

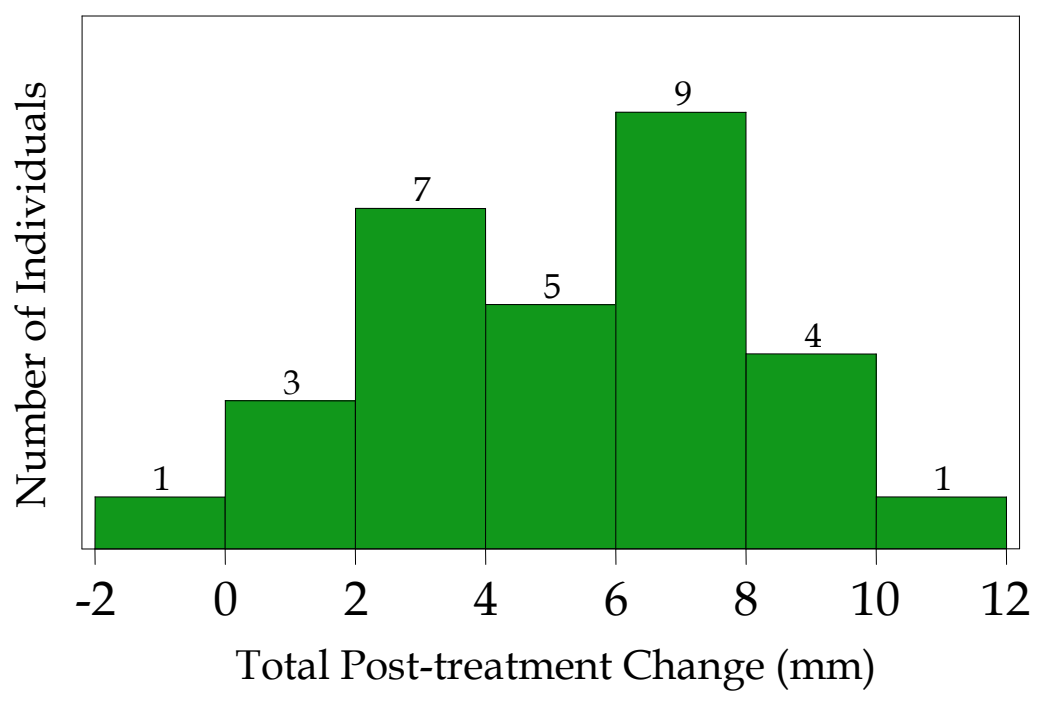

Figure C-20. Plot of the distribution of posttreatment changes in CondylionGonion distance among the 30 women in the sample. Numbers atop each bar are the numbers of cases in that interval. 


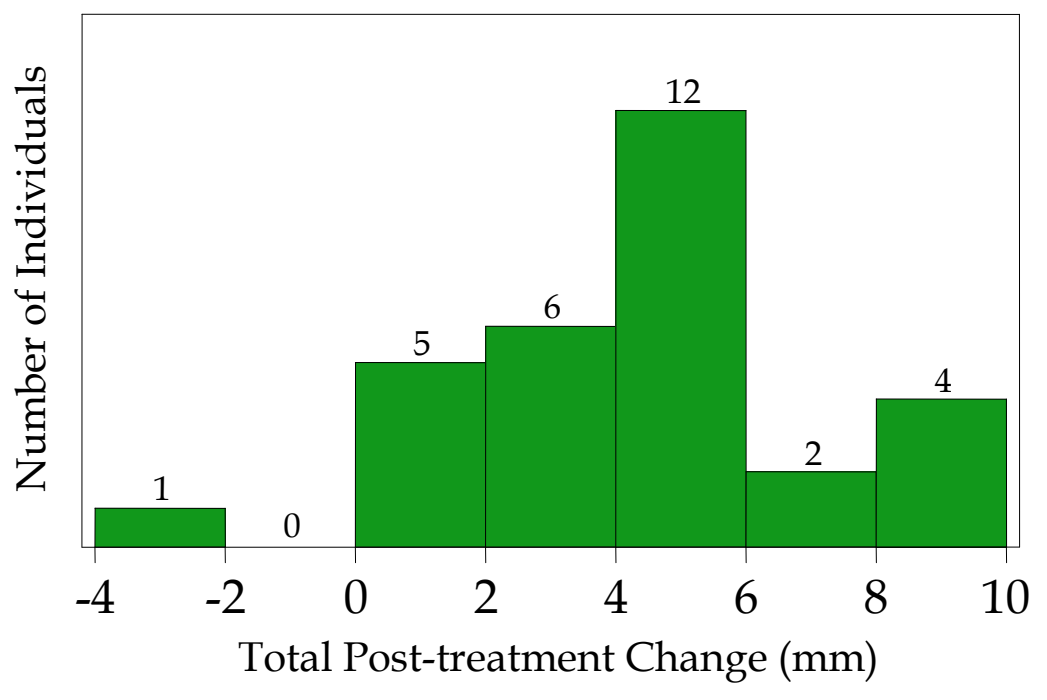

Figure C-21. Plot of the distribution of posttreatment changes in GonionPogonion distance among the 30 women in the sample. Numbers atop each bar are the numbers of cases in that interval.

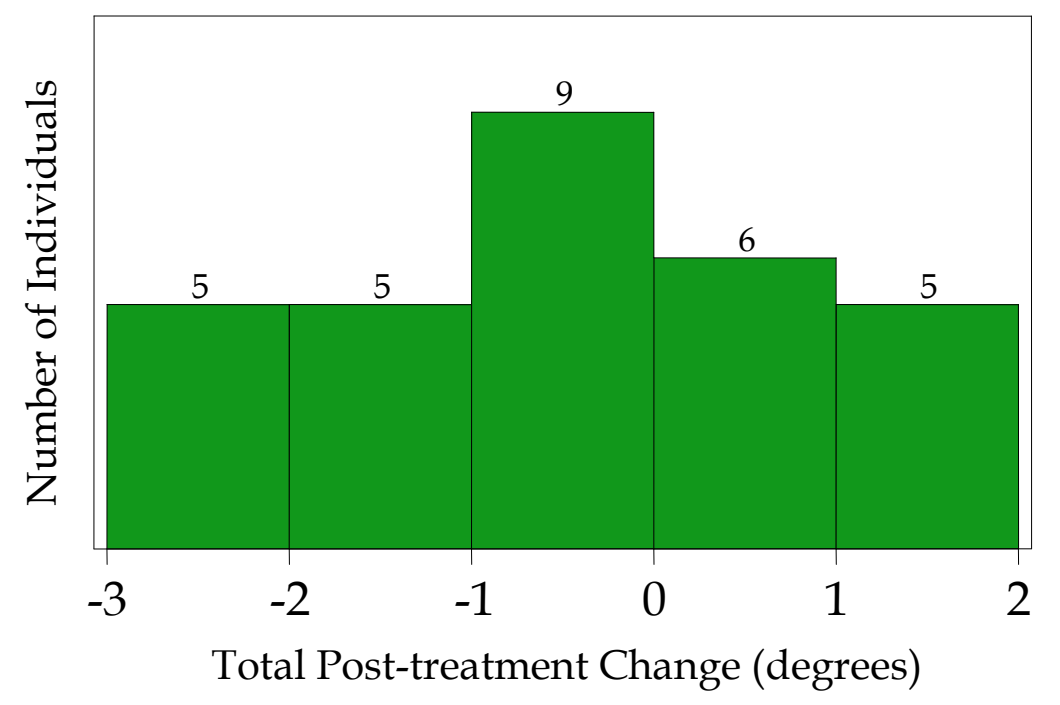

Figure C-22. Plot of the distribution of posttreatment changes in Y-Axis angle among the 30 women in the sample. Numbers atop each bar are the numbers of cases in that interval. 


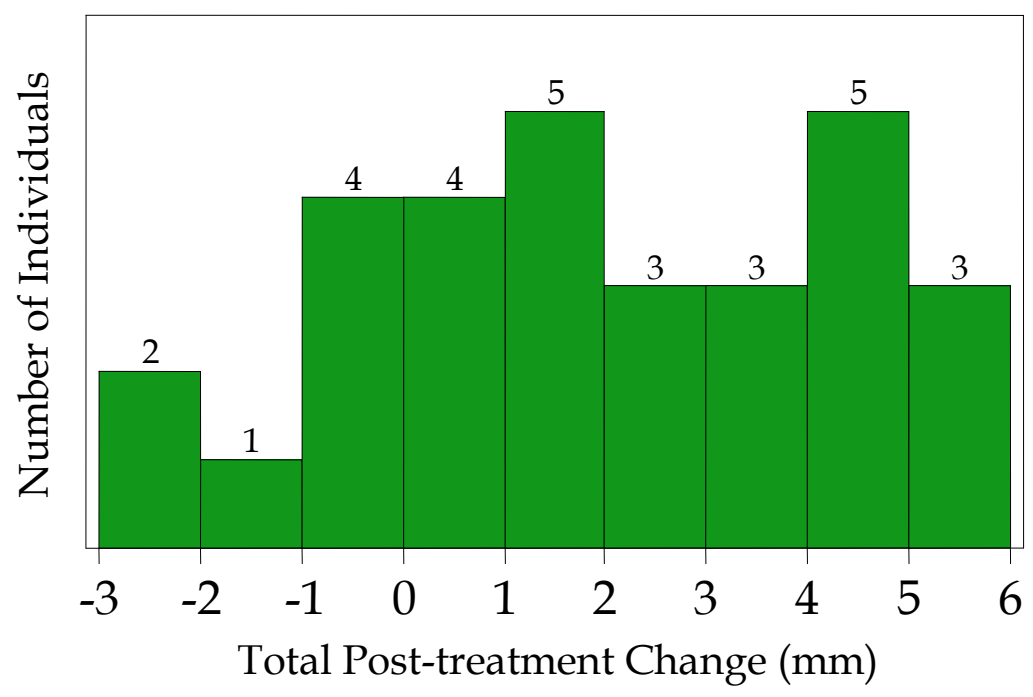

Figure C-23. Plot of the distribution of posttreatment changes in Nasion Perpendicular-Pogonion distance among the 30 women in the sample. Numbers atop each bar are the numbers of cases in that interval.

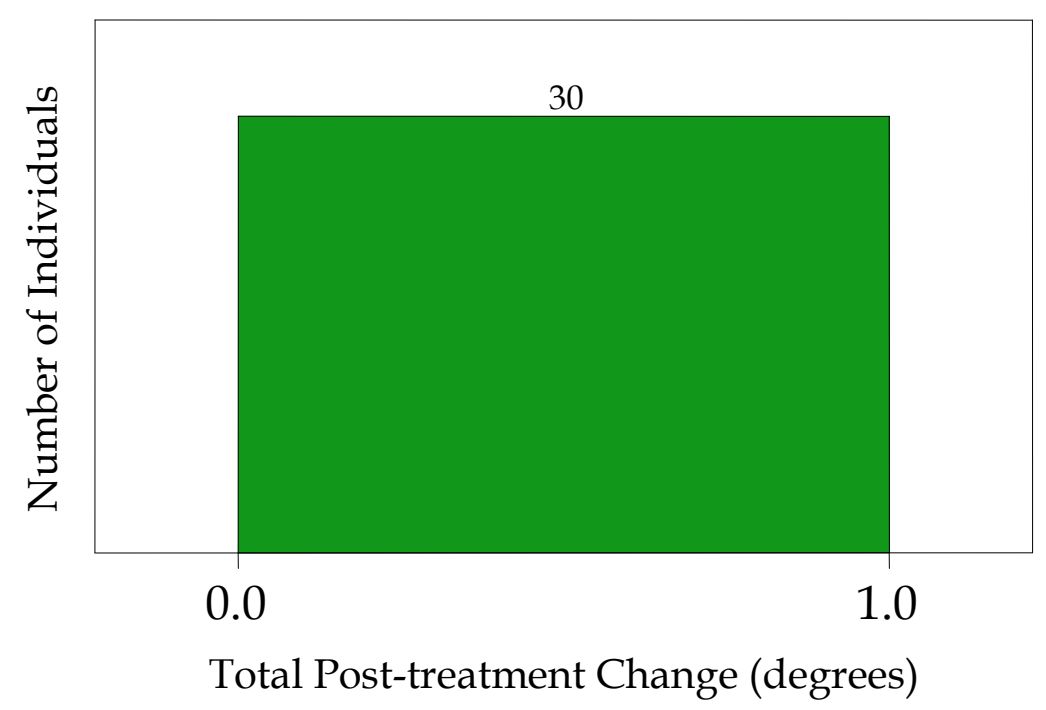

Figure C-24. Plot of the distribution of posttreatment changes in A Point-Nasion$B$ Point angle among the 30 women in the sample. Numbers atop each bar are the numbers of cases in that interval. 


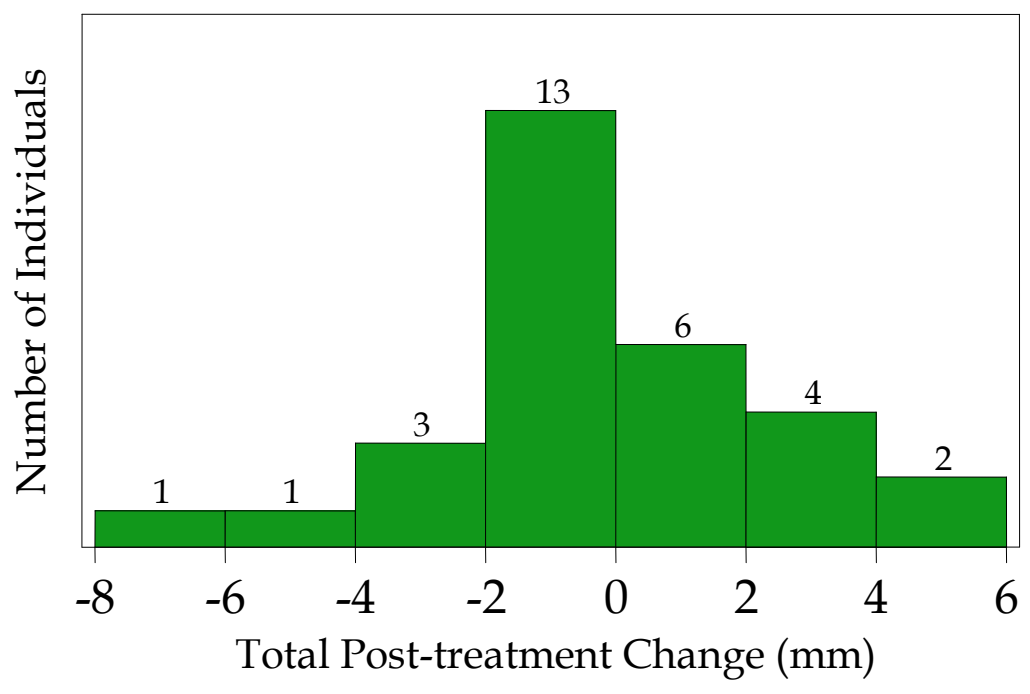

Figure C-25. Plot of the distribution of posttreatment changes in Wits Appraisal distance among the 30 women in the sample. Numbers atop each bar are the numbers of cases in that interval.

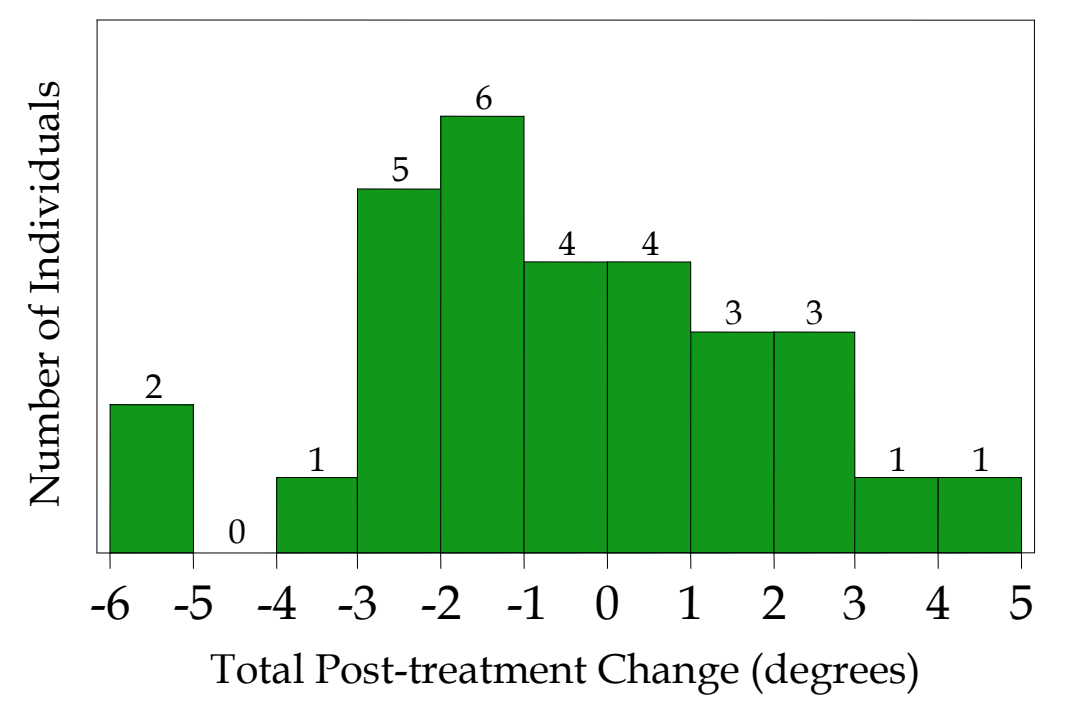

Figure C-26. Plot of the distribution of posttreatment changes in Frankfort Horizontal-Mandibular Plane Angle among the 30 women in the sample. Numbers atop each bar are the numbers of cases in that interval. 


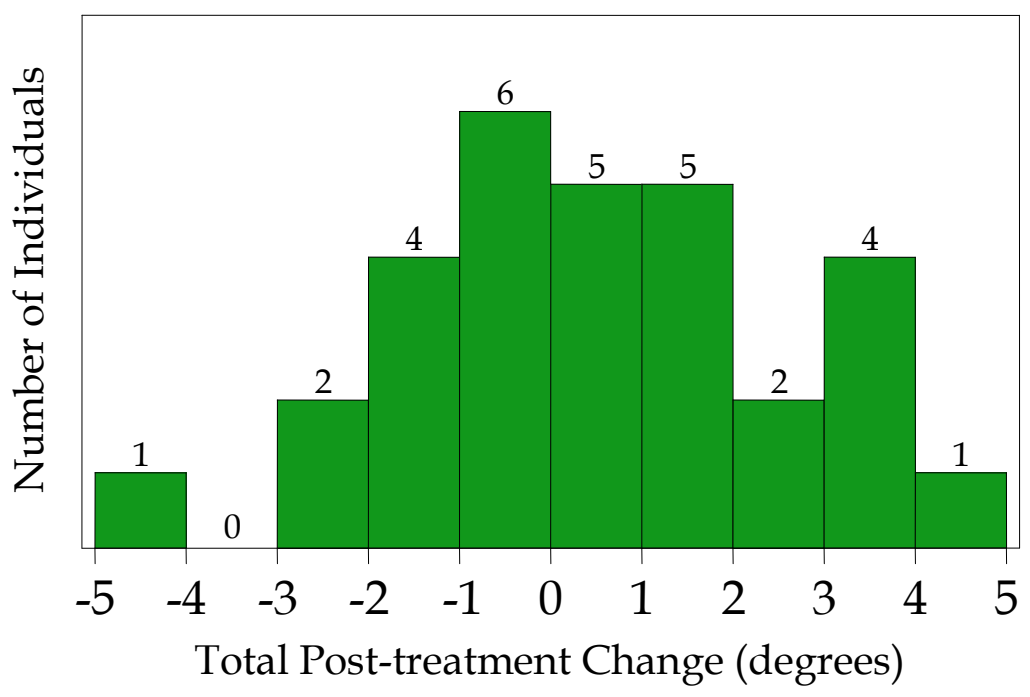

Figure C-27. Plot of the distribution of posttreatment changes in Facial Convexity (Nasion-A Point- Pogonion angle) among the 30 women in the sample. Numbers atop each bar are the numbers of cases in that interval.

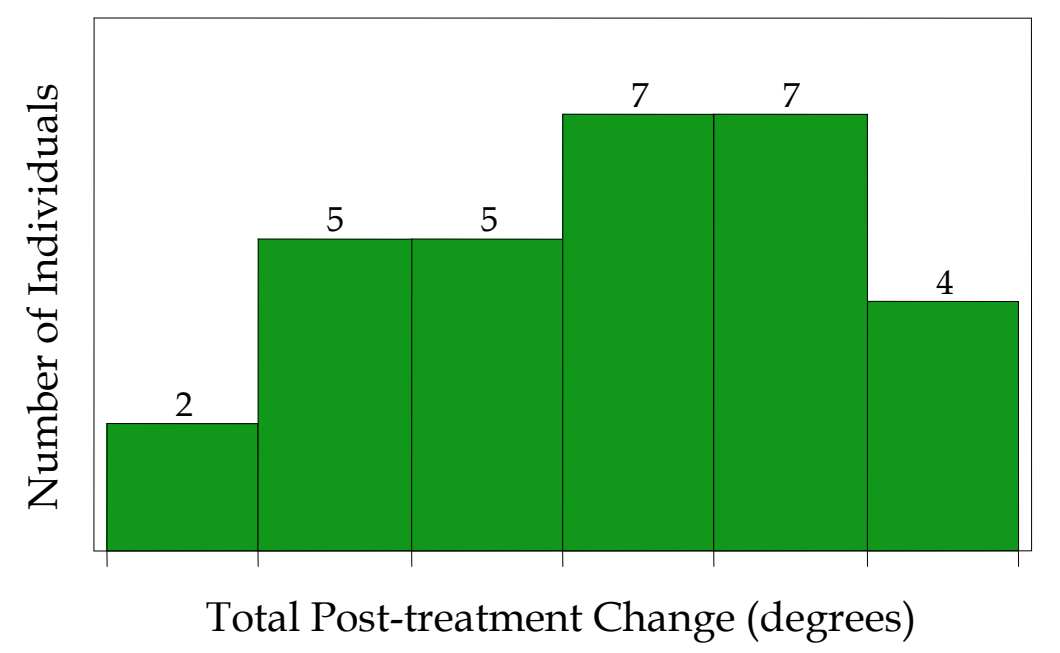

Figure C-28. Plot of the distribution of posttreatment changes in Frankfort Horizontal to Nasion-Pogonion angle among the 30 women in the sample. Numbers atop each bar are the numbers of cases in that interval. 


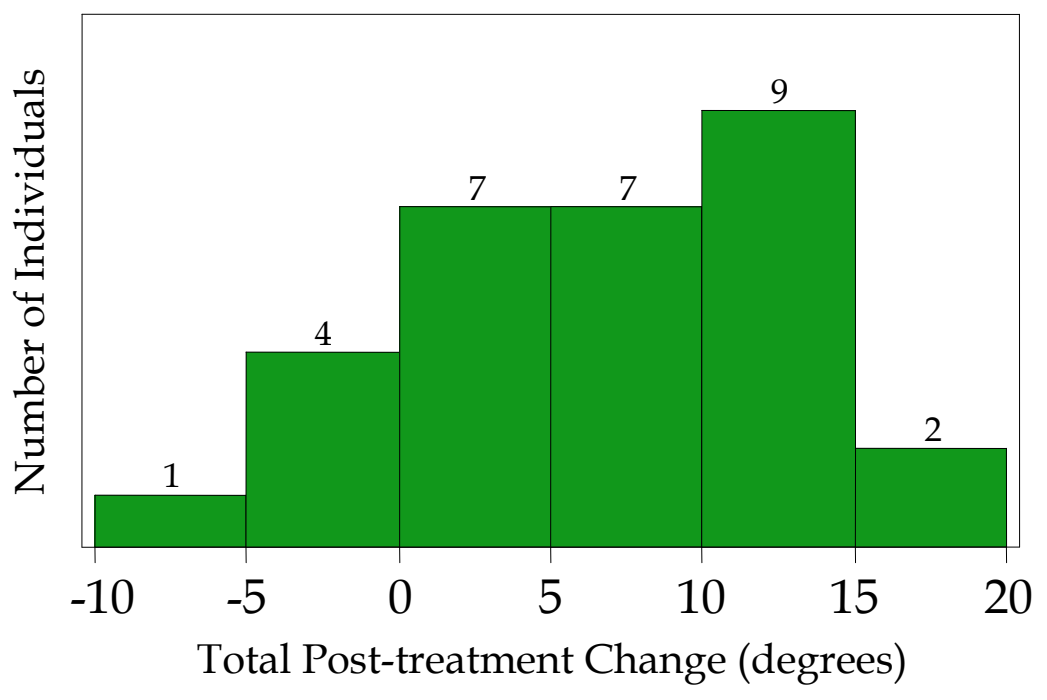

Figure C-29. Plot of the distribution of posttreatment changes in Interincisal Angle (Upper 1/Lower 1) among the 30 women in the sample. Numbers atop each bar are the numbers of cases in that interval.

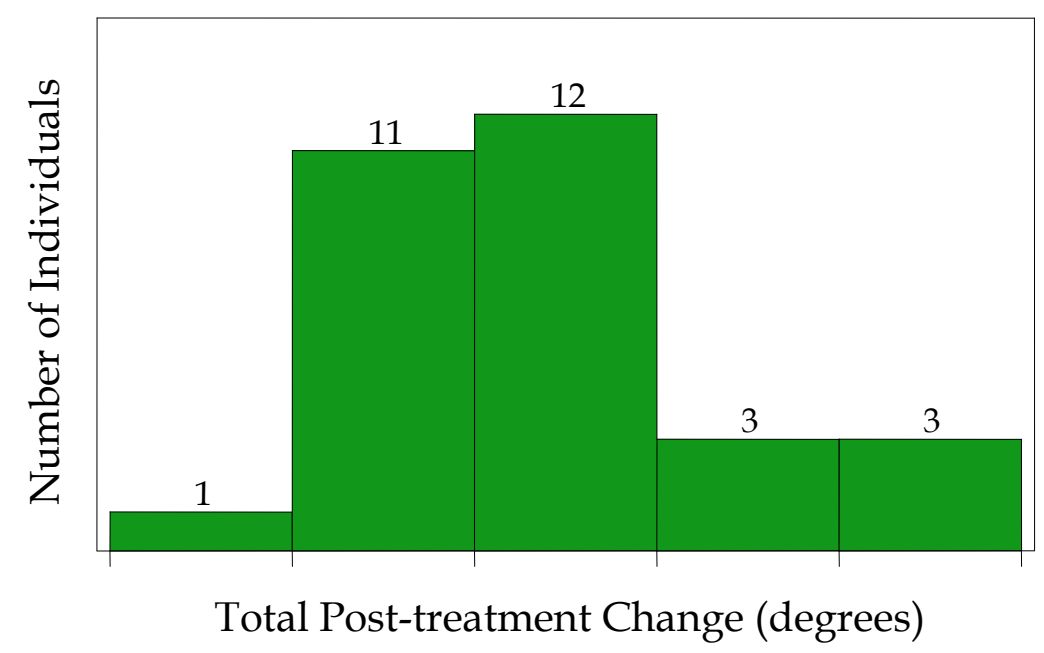

Figure C-30. Plot of the distribution of posttreatment changes in Upper 1 to SellaNasion angle among the 30 women in the sample. Numbers atop each bar are the numbers of cases in that interval. 


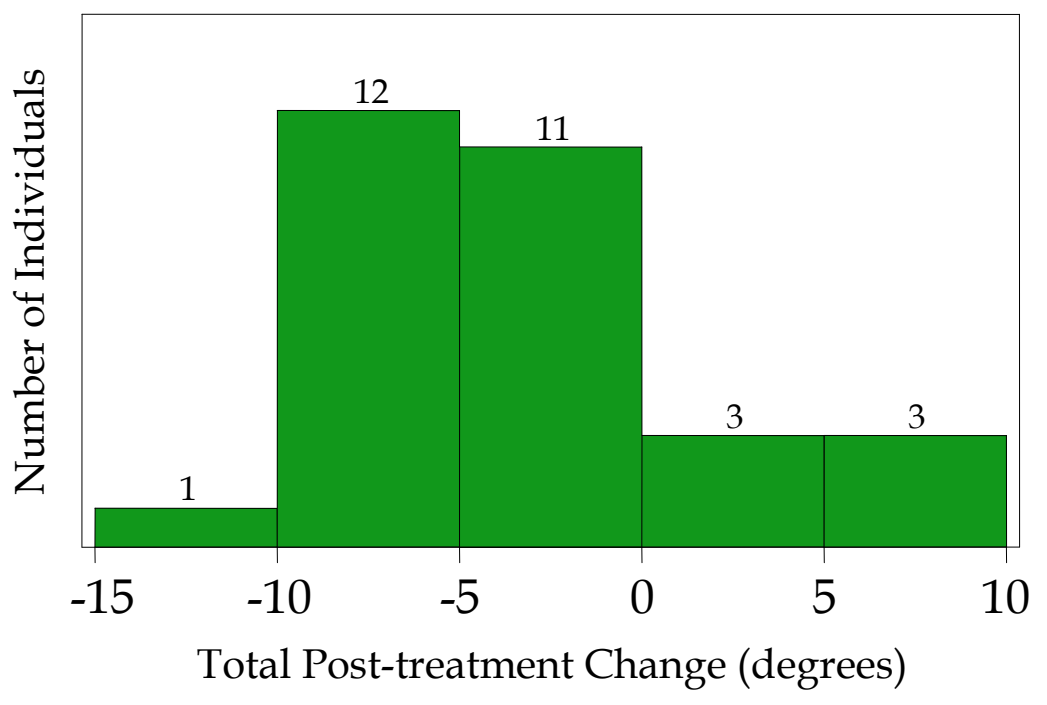

Figure C-31. Plot of the distribution of posttreatment changes in Upper 1 to Nasion-A point angle among the 30 women in the sample. Numbers atop each bar are the numbers of cases in that interval.

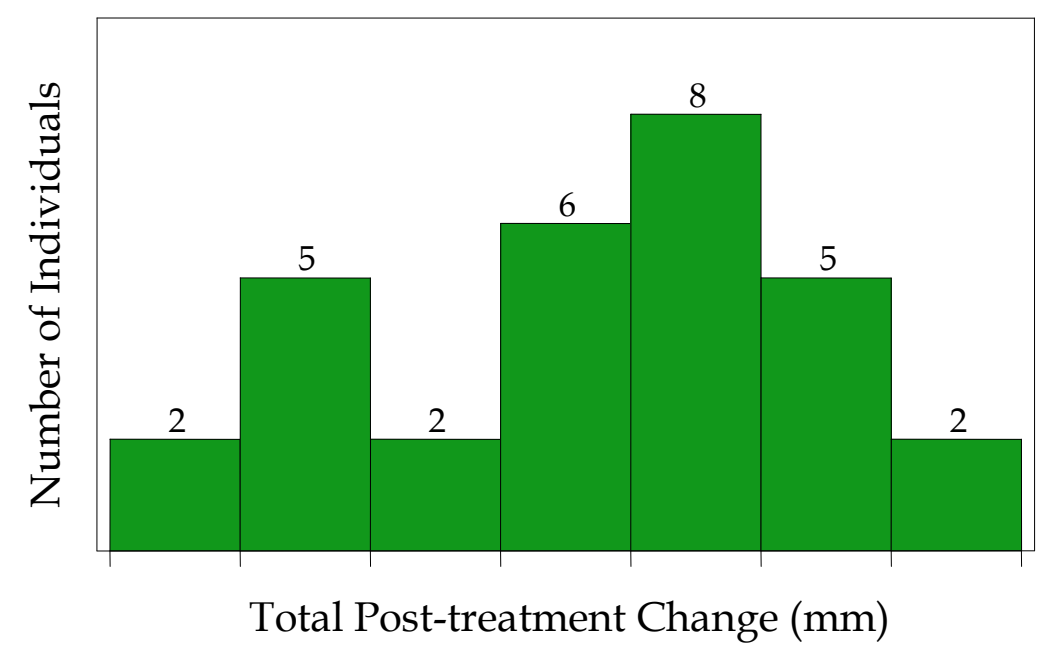

Figure C-32. Plot of the distribution of posttreatment changes in Upper 1 to Nasion-A point distance among the 30 women in the sample. Numbers atop each bar are the numbers of cases in that interval. 


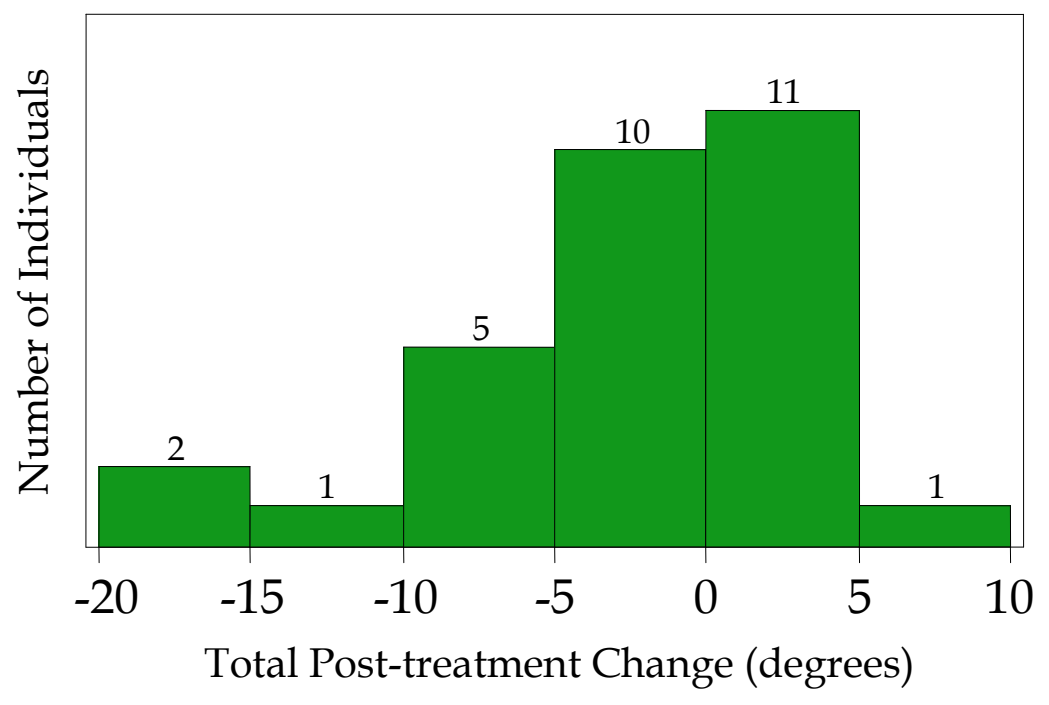

Figure C-33. Plot of the distribution of posttreatment changes in Lower 1 to Nasion-B point angle among the 30 women in the sample. Numbers atop each bar are the numbers of cases in that interval.

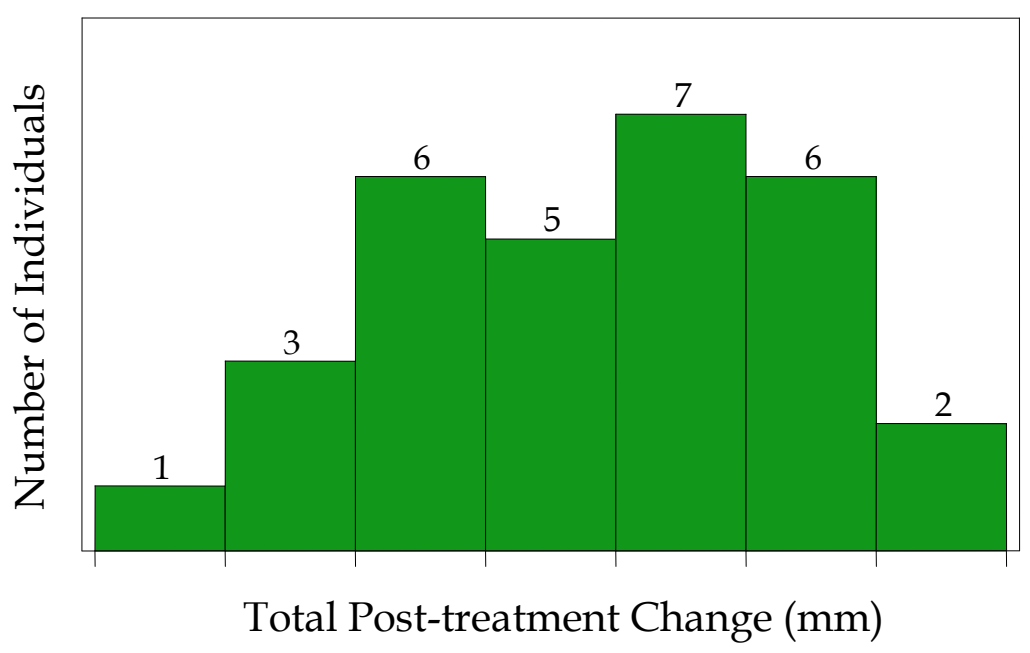

Figure C-34. Plot of the distribution of posttreatment changes in Lower 1 to Nasion-B point distance among the 30 women in the sample. Numbers atop each bar are the numbers of cases in that interval. 


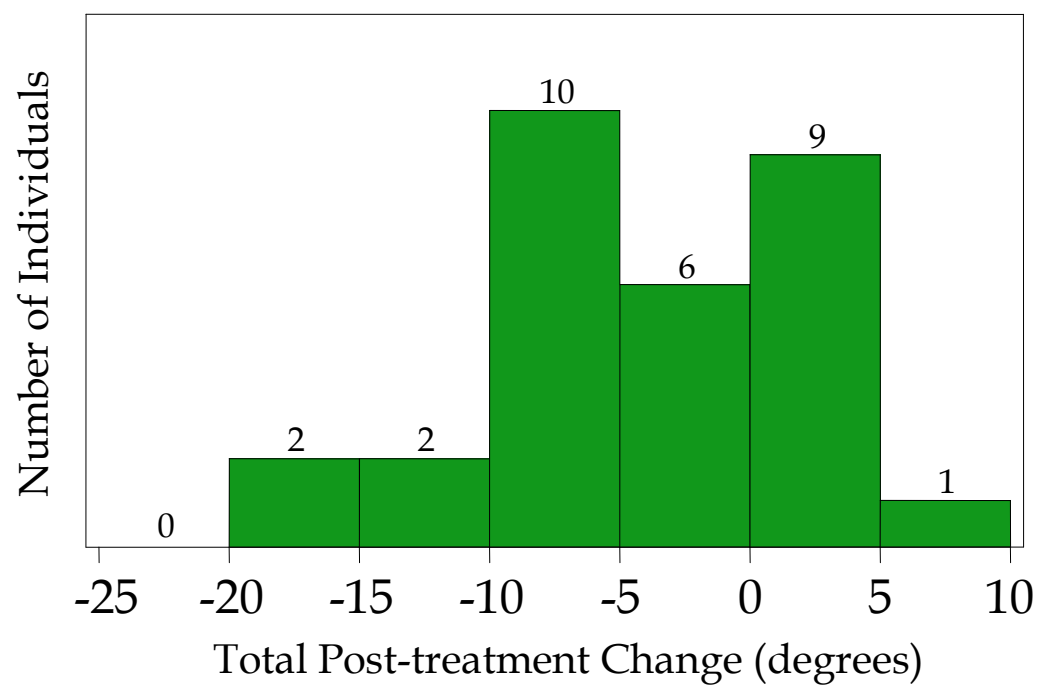

Figure C-35. Plot of the distribution of posttreatment changes in Lower IncisorMandibular Plane angle among the 30 women in the sample. Numbers atop each bar are the numbers of cases in that interval.

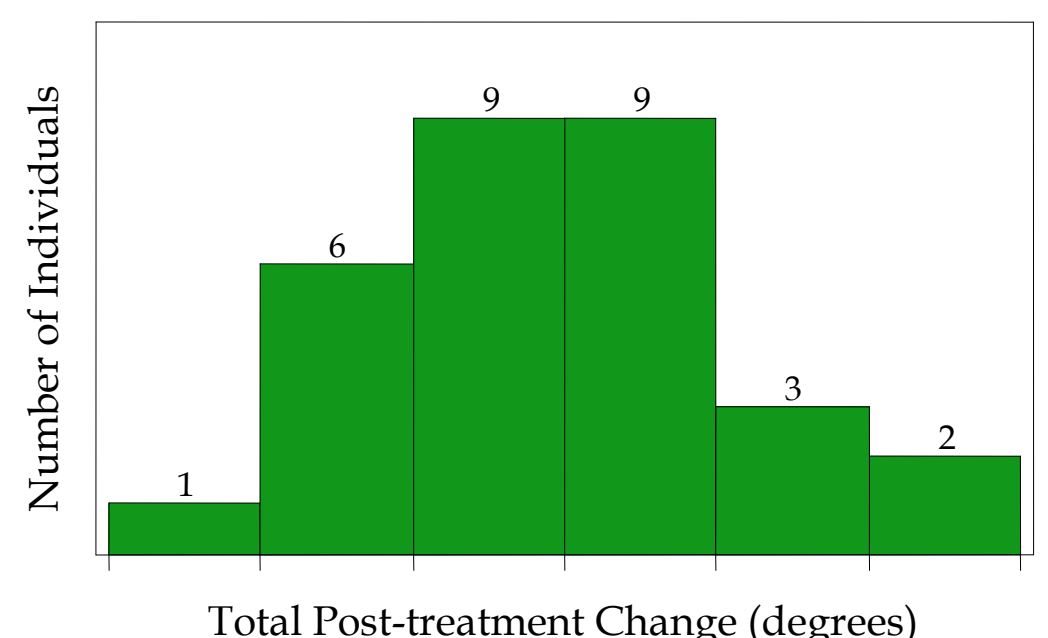

Figure C-36. Plot of the distribution of posttreatment changes in Frankfort Horizontal-Mandibular Incisor angle among the 30 women in the sample. Numbers atop each bar are the numbers of cases in that interval. 


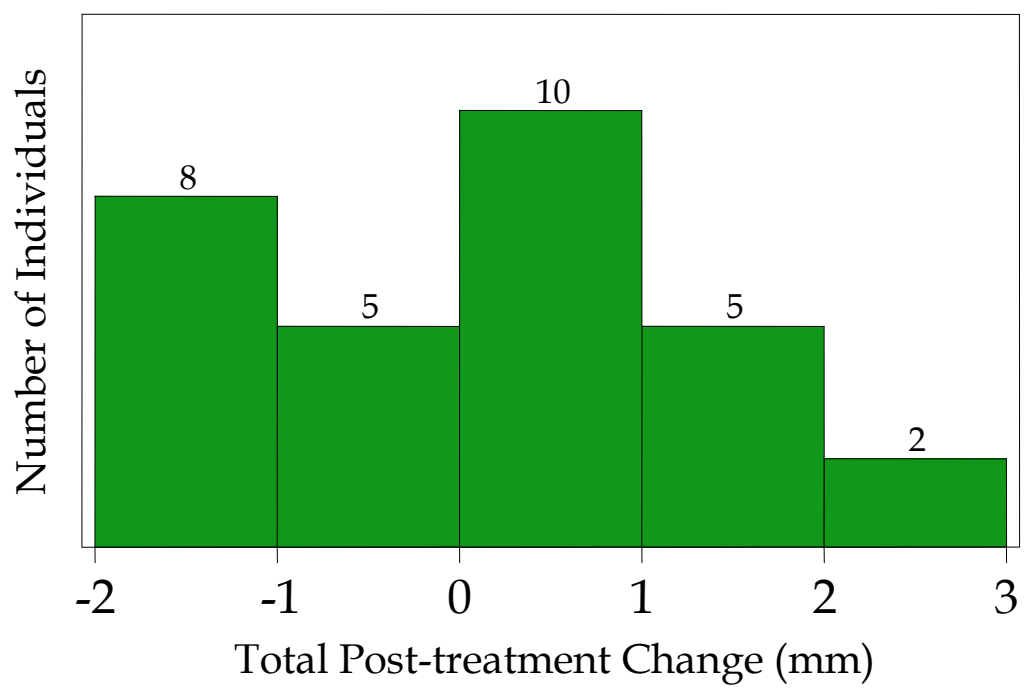

Figure C-37. Plot of the distribution of posttreatment changes in Upper 1 to A point-Pogonion distance among the 30 women in the sample. Numbers atop each bar are the numbers of cases in that interval.

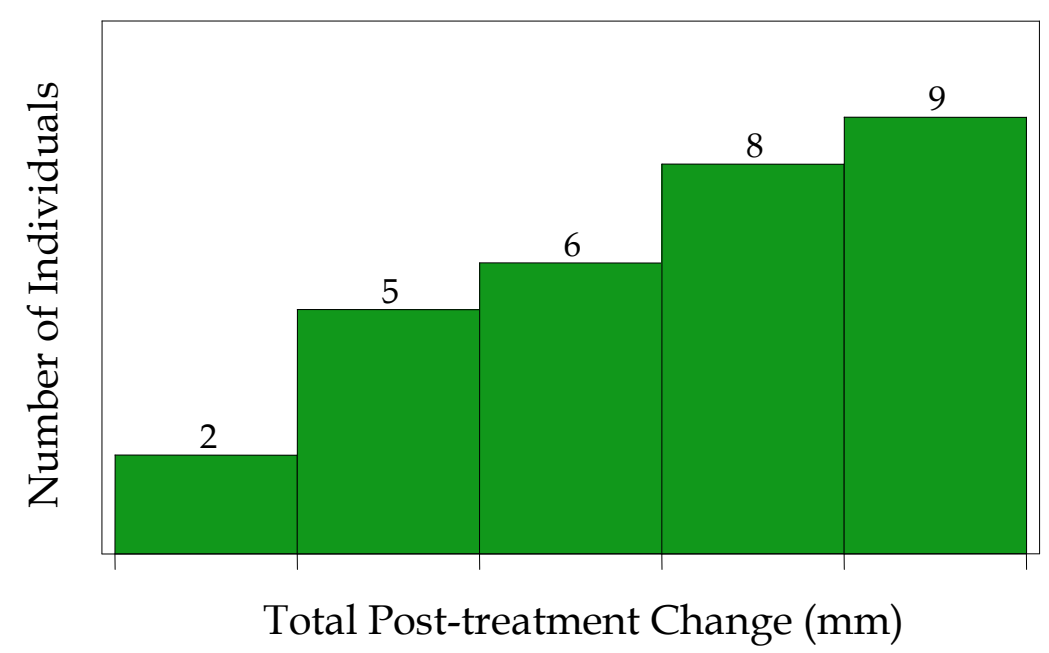

Figure C-38. Plot of the distribution of posttreatment changes in Lower 1 to A point-Pogonion distance among the 30 women in the sample. Numbers atop each bar are the numbers of cases in that interval. 


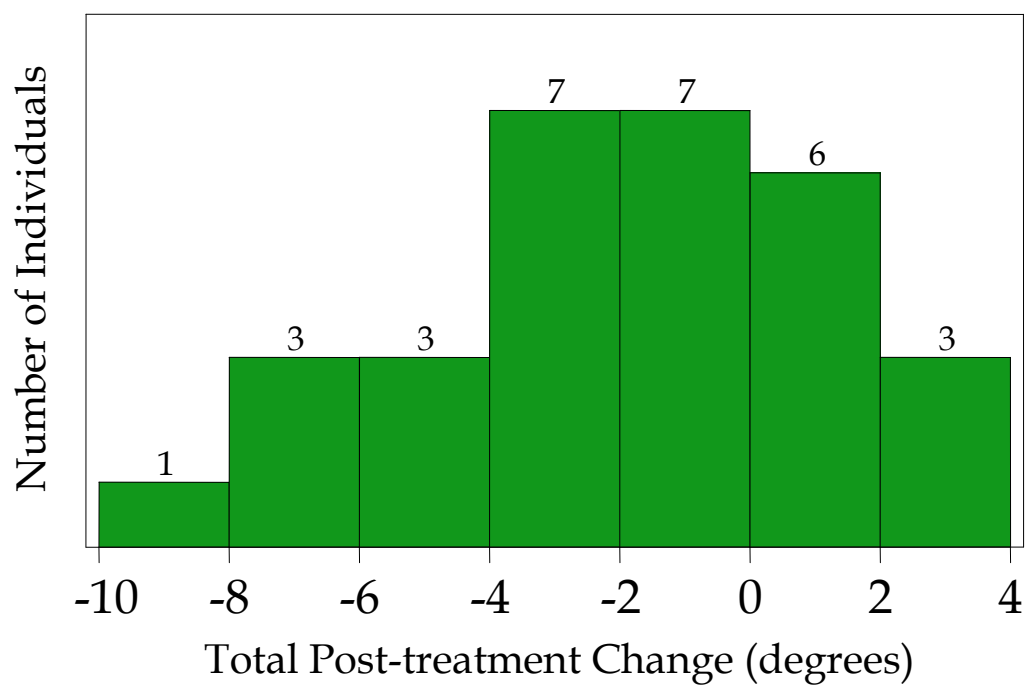

Figure C-39. Plot of the distribution of posttreatment changes in Occlusal PlaneFrankfort Horizontal Plane angle among the 30 women in the sample. Numbers atop each bar are the numbers of cases in that interval.

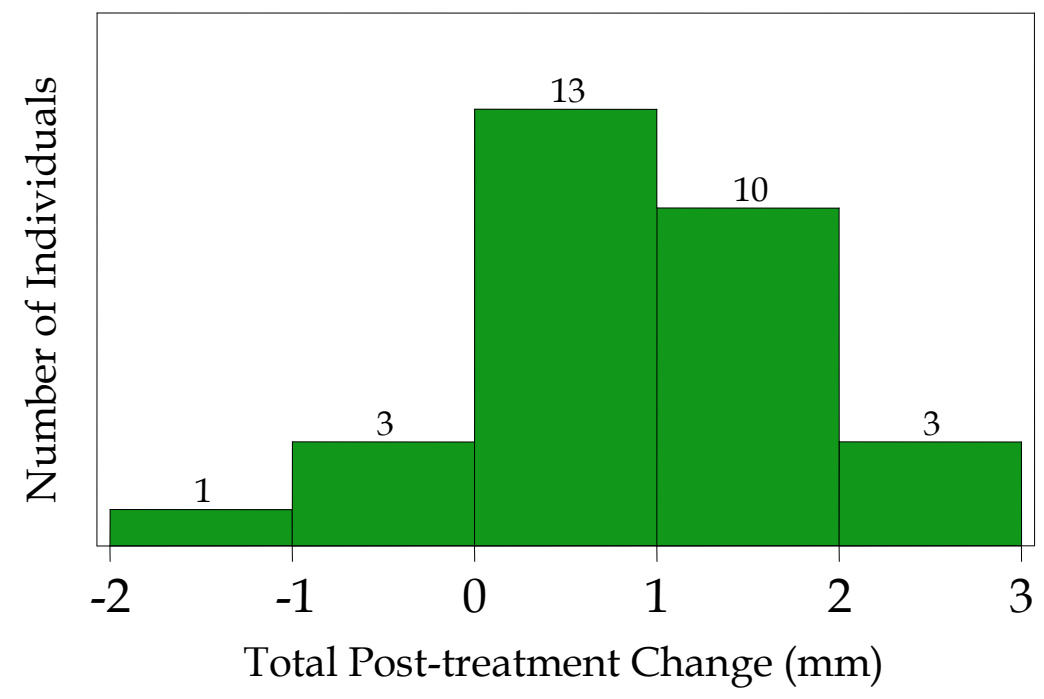

Figure C-40. Plot of the distribution of posttreatment changes in Overbite distance among the 30 women in the sample. Numbers atop each bar are the numbers of cases in that interval. 


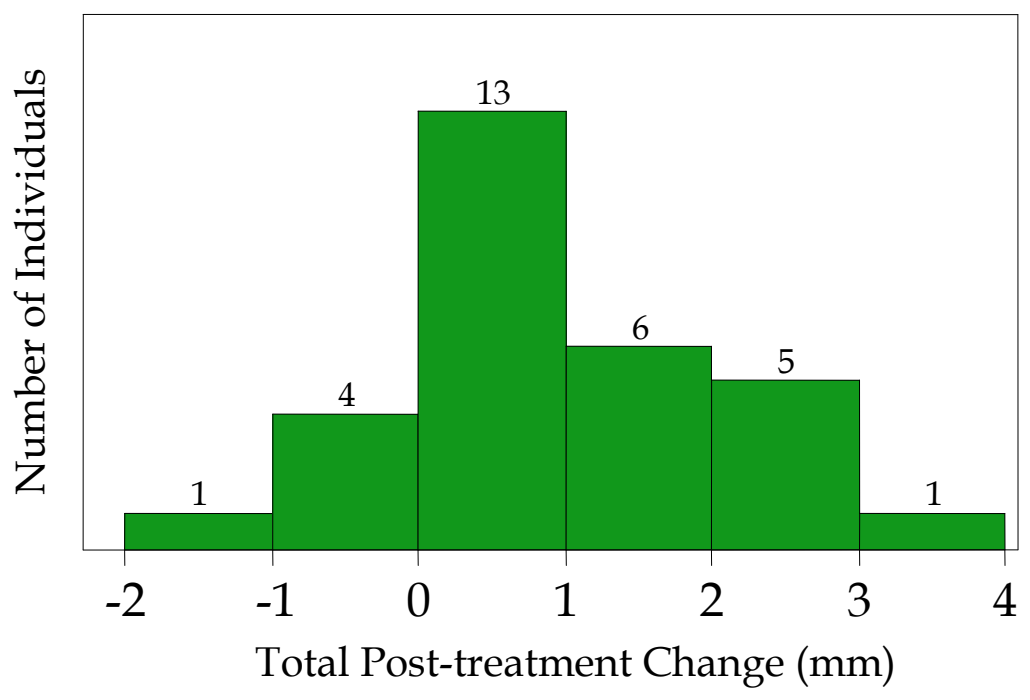

Figure C-41. Plot of the distribution of posttreatment changes in Overjet distance among the 30 women in the sample. Numbers atop each bar are the numbers of cases in that interval.

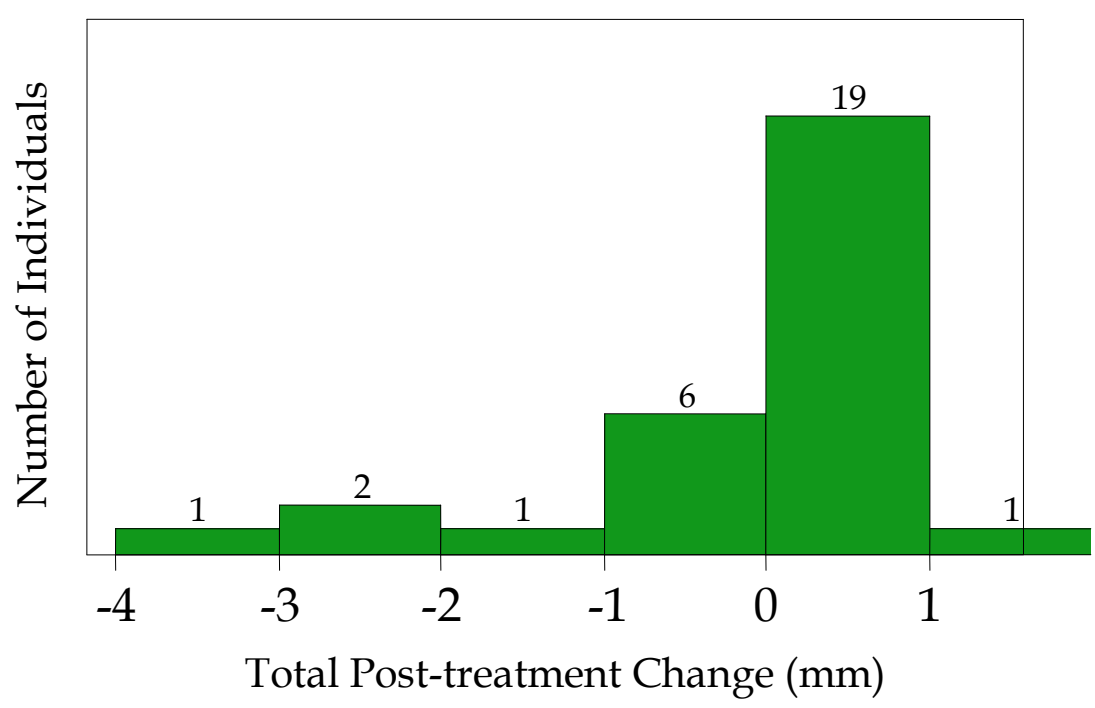

Figure C-42. Plot of the distribution of posttreatment changes in Lower 1-W point distance among the 30 women in the sample. Numbers atop each bar are the numbers of cases in that interval. 


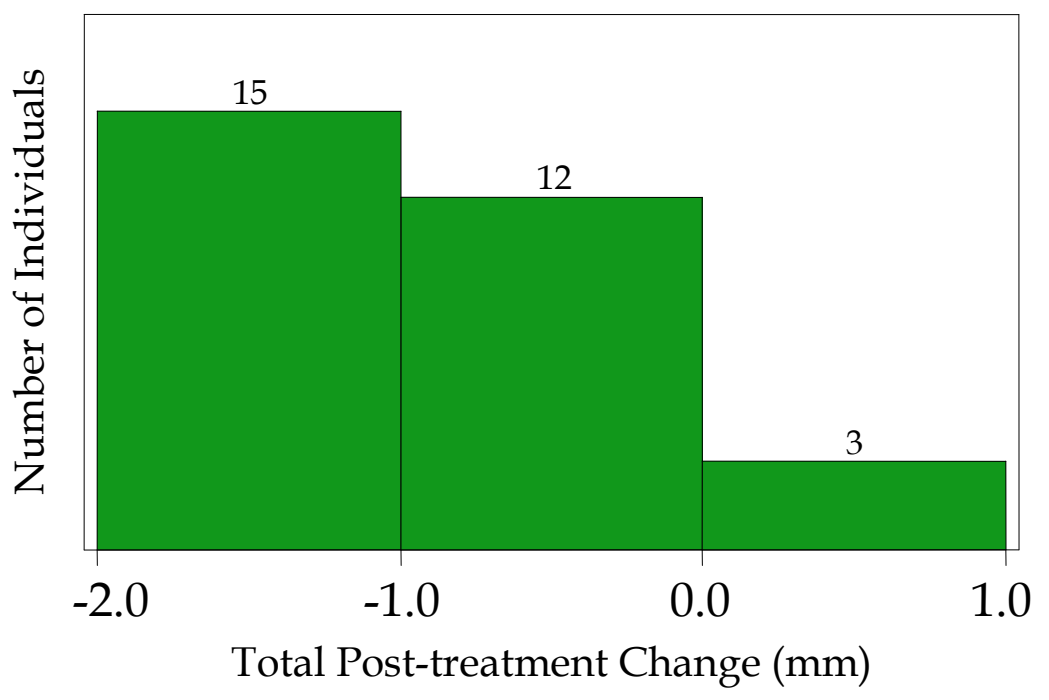

Figure C-43. Plot of the distribution of posttreatment changes in Distal Lower 6$\mathrm{W}$ point distance among the 30 women in the sample. Numbers atop each bar are the numbers of cases in that interval.

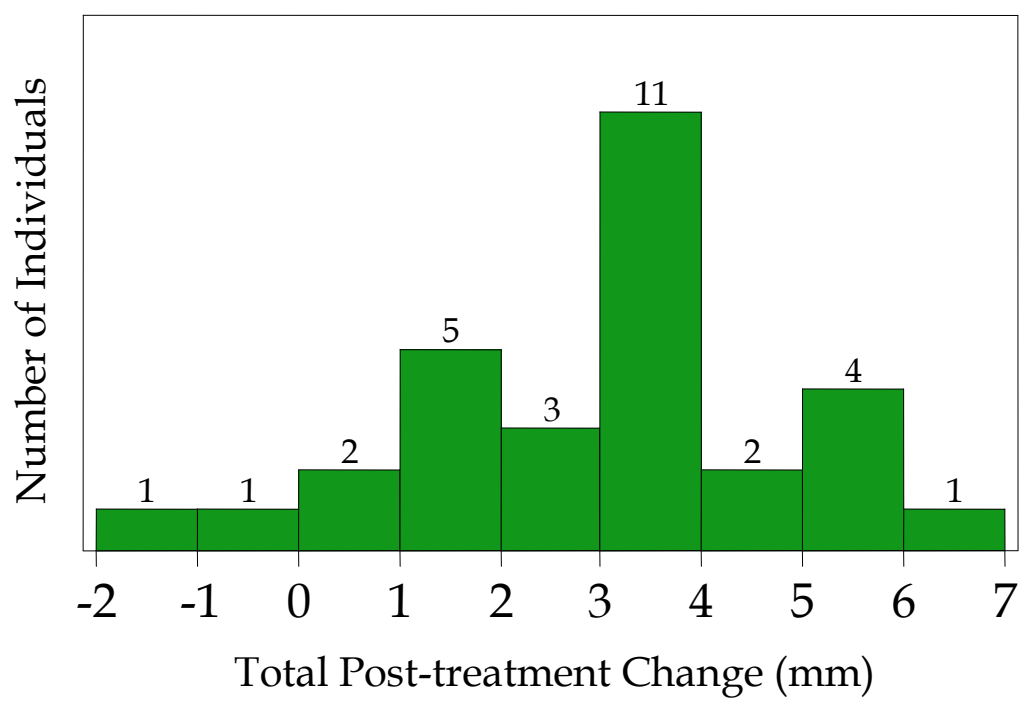

Figure C-44. Plot of the distribution of posttreatment changes in Upper 1Pterygoid Vertical distance among the 30 women in the sample. Numbers atop each bar are the numbers of cases in that interval. 


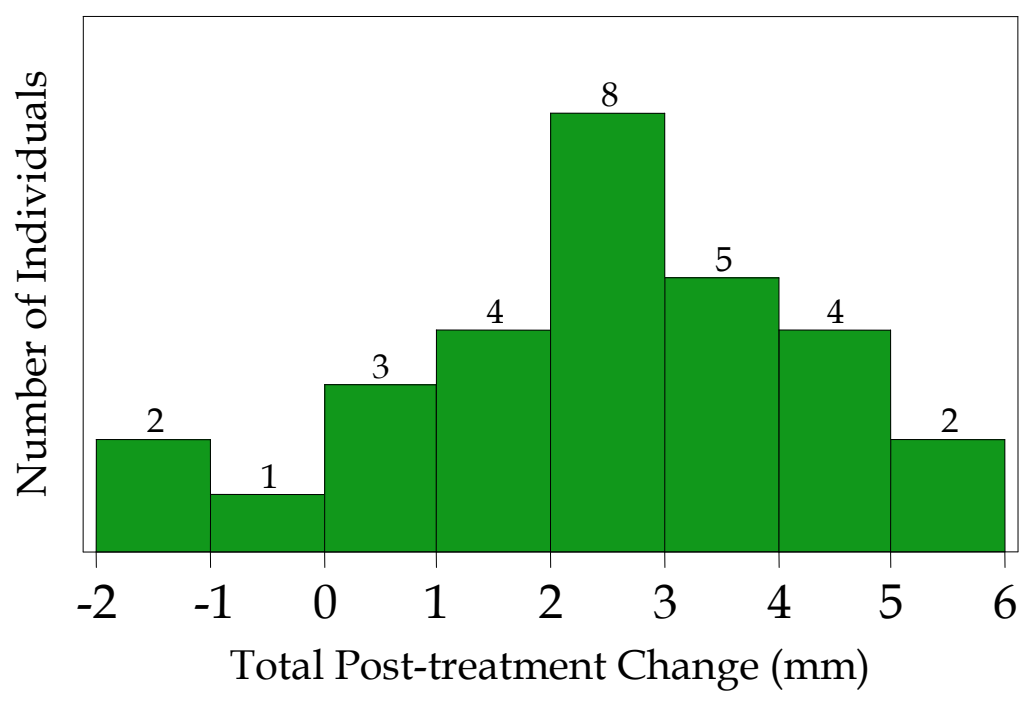

Figure C-45. Plot of the distribution of posttreatment changes in Upper 6Pterygoid Vertical distance among the 30 women in the sample. Numbers atop each bar are the numbers of cases in that interval.

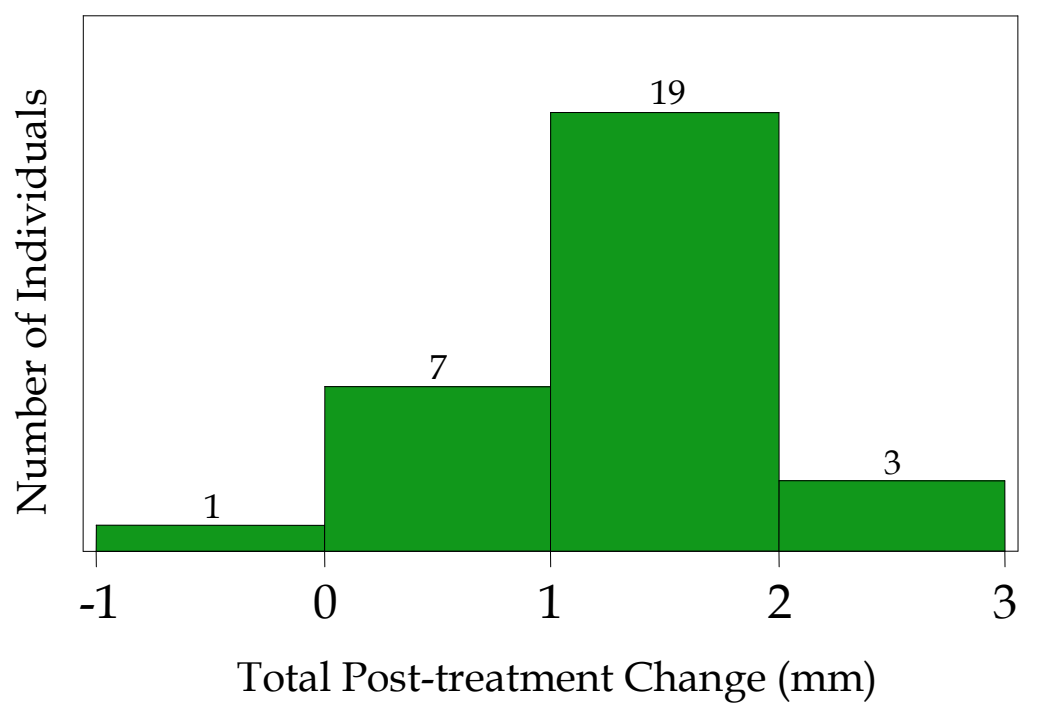

Figure C-46. Plot of the distribution of posttreatment changes in Mandibular Arch Length (Distal Lower 6-L1) among the 30 women in the sample. Numbers atop each bar are the numbers of cases in that interval. 


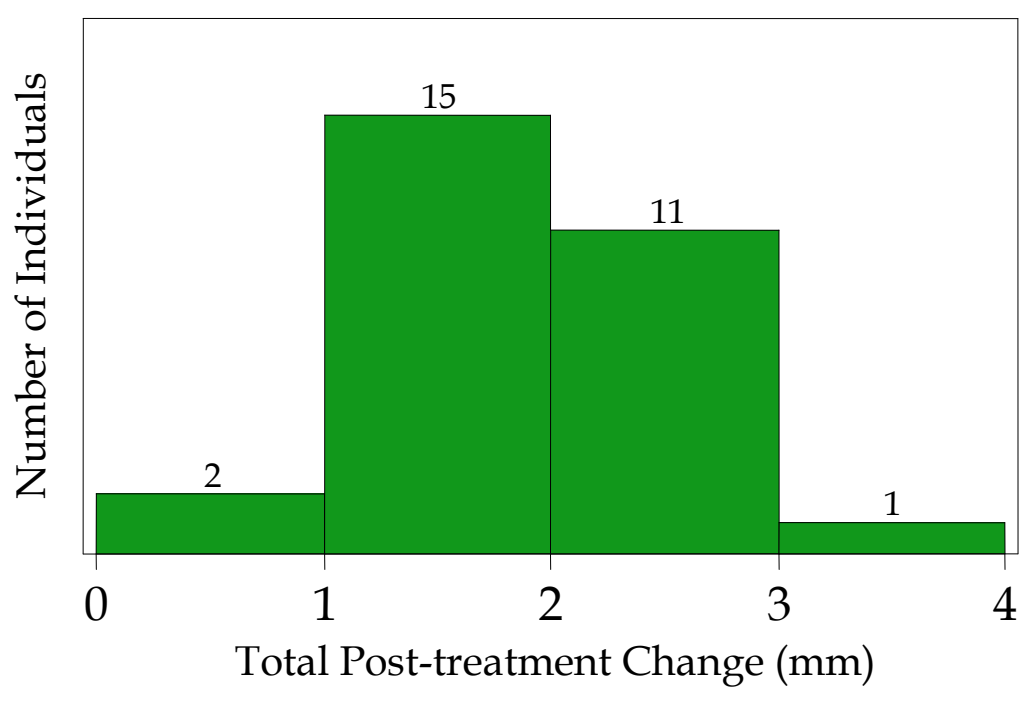

Figure C-47. Plot of the distribution of posttreatment changes in Maxillary Arch Length (Distal U6-U1) among the 30 women in the sample. Numbers atop each bar are the numbers of cases in that interval.

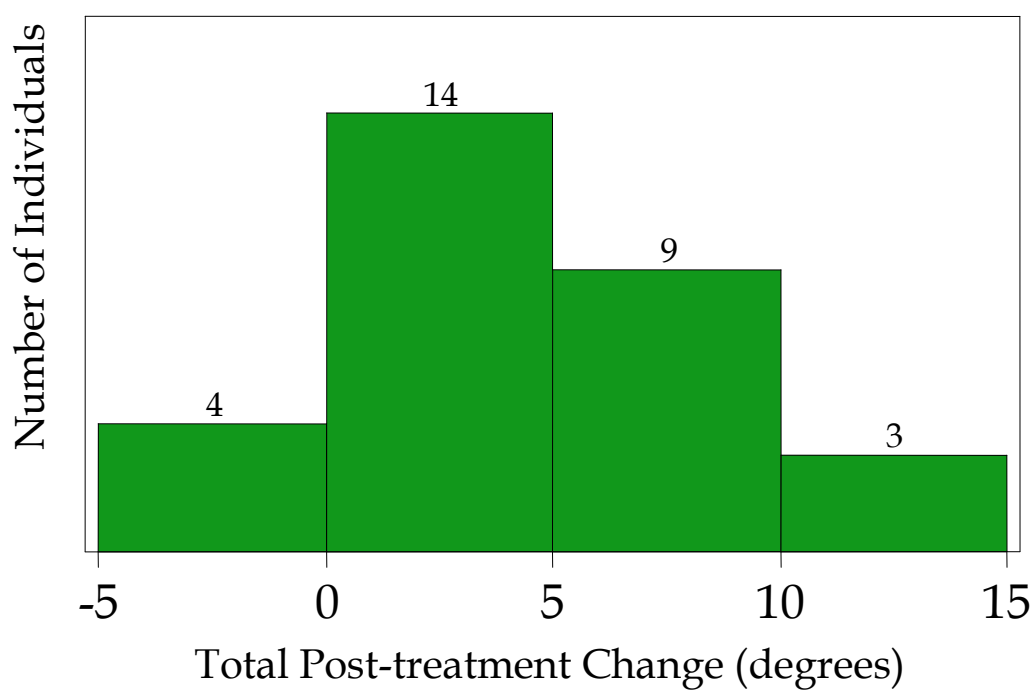

Figure C-48. Plot of the distribution of posttreatment changes in Z Angle among the 30 women in the sample. Numbers atop each bar are the numbers of cases in that interval. 


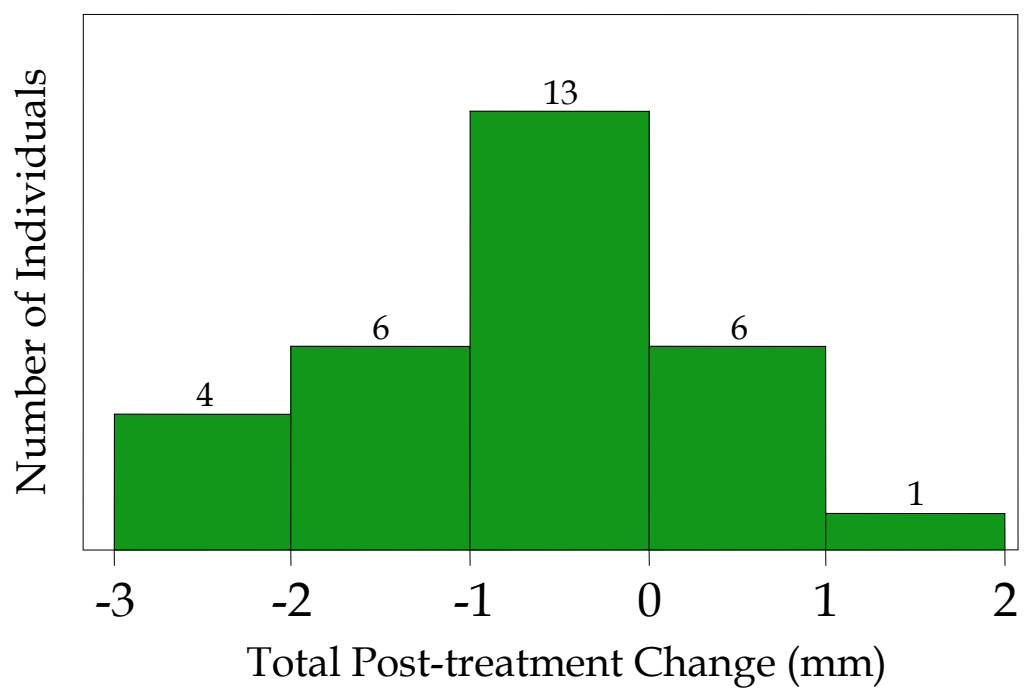

Figure C-49. Plot of the distribution of posttreatment changes in E Plane-Labrale Superius distance among the 30 women in the sample. Numbers atop each bar are the numbers of cases in that interval.

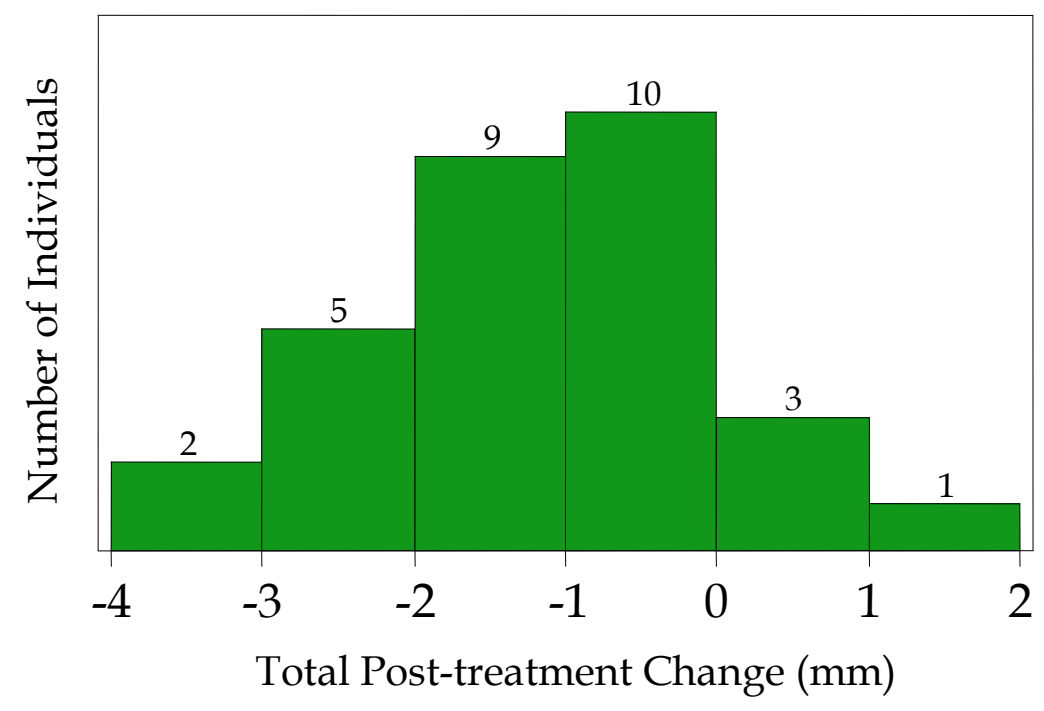

Figure C-50. Plot of the distribution of posttreatment changes in E Plane-Labrale Inferius distance among the 30 women in the sample. Numbers atop each bar are the numbers of cases in that interval. 


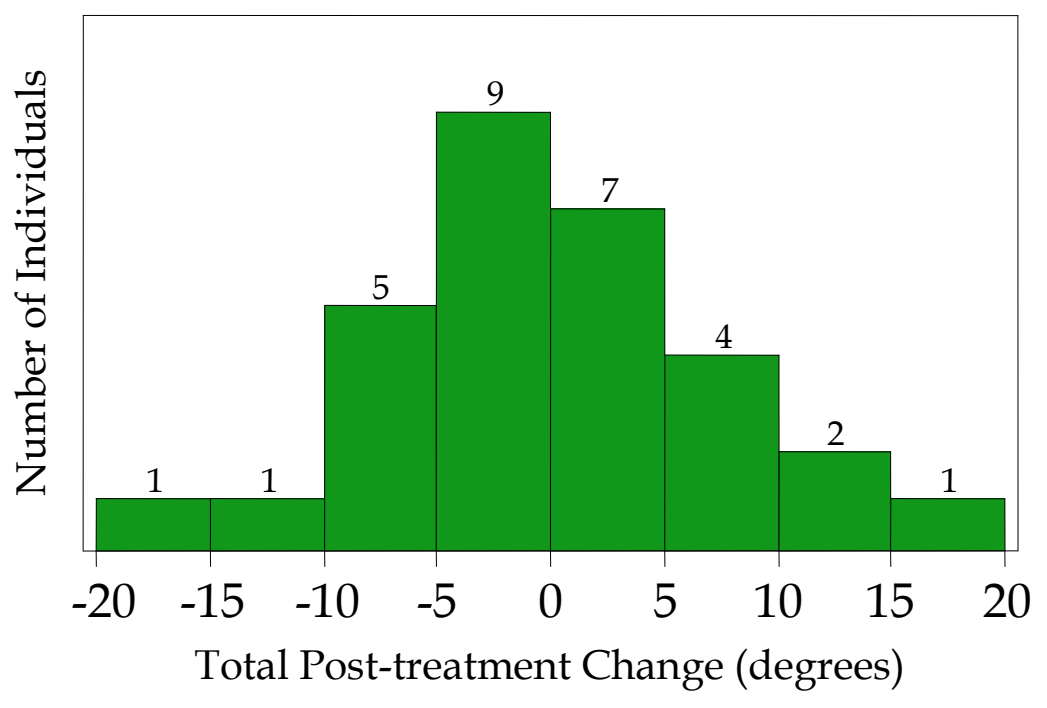

Figure C-51. Plot of the distribution of posttreatment changes in Nasolabial Angle (Pronasale-Subnasale-Labrale Superius) among the 30 women in the sample. Numbers atop each bar are the numbers of cases in that interval.

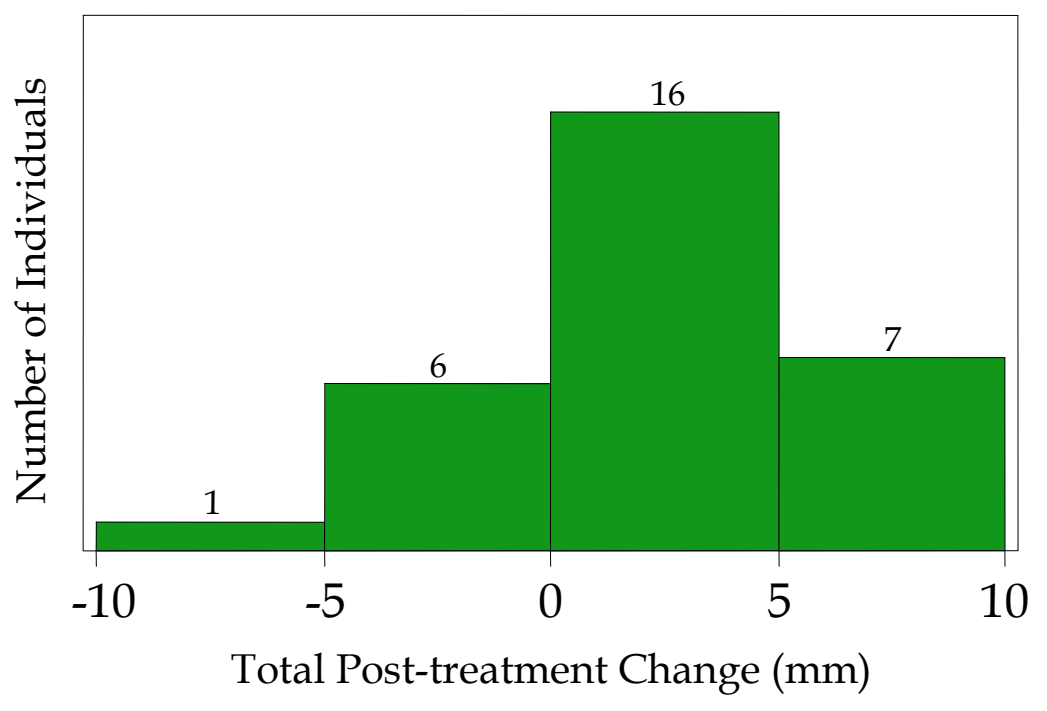

Figure C-52. Plot of the distribution of posttreatment changes in Mentolabial Angle (Labrale Inferius-Soft Tissue B Point-Soft Tissue Pogonion) distance among the 30 women in the sample. Numbers atop each bar are the numbers of cases in that interval. 


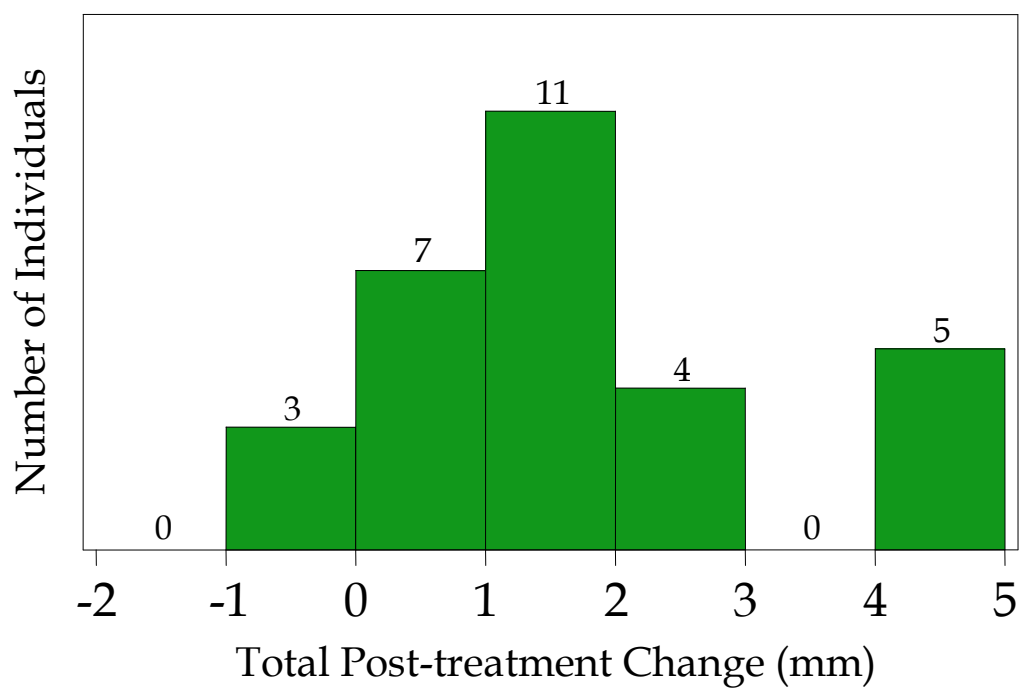

Figure C-53. Plot of the distribution of posttreatment changes in SubnasaleLabrale Superius distance among the 30 women in the sample. Numbers atop each bar are the numbers of cases in that interval.

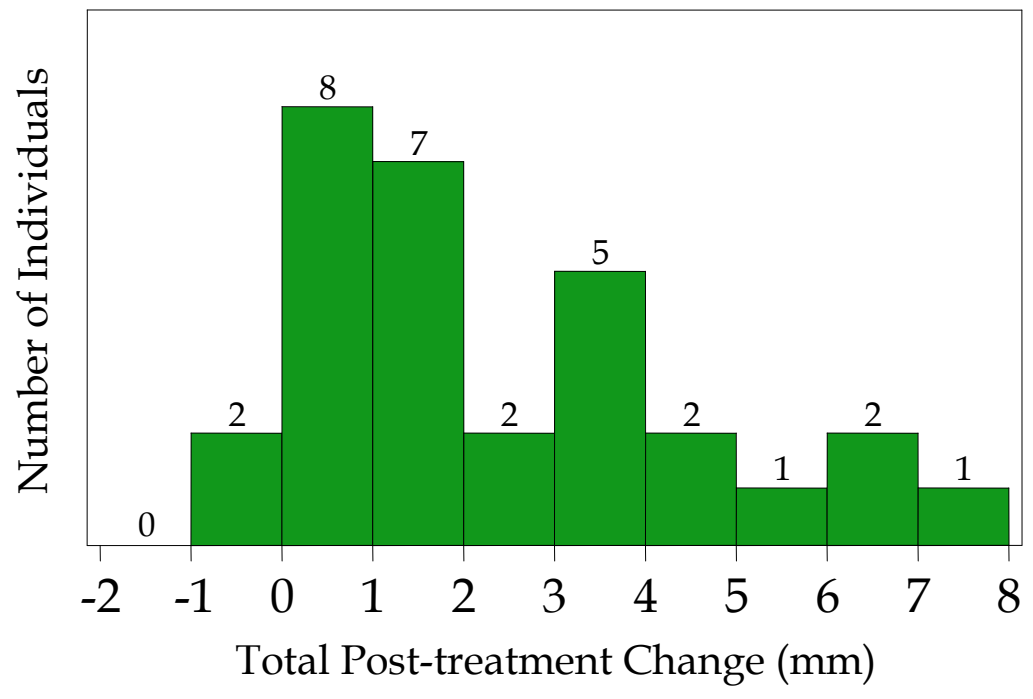

Figure C-54. Plot of the distribution of posttreatment changes in SubnasaleLabrale Inferius distance among the 30 women in the sample. Numbers atop each bar are the numbers of cases in that interval. 


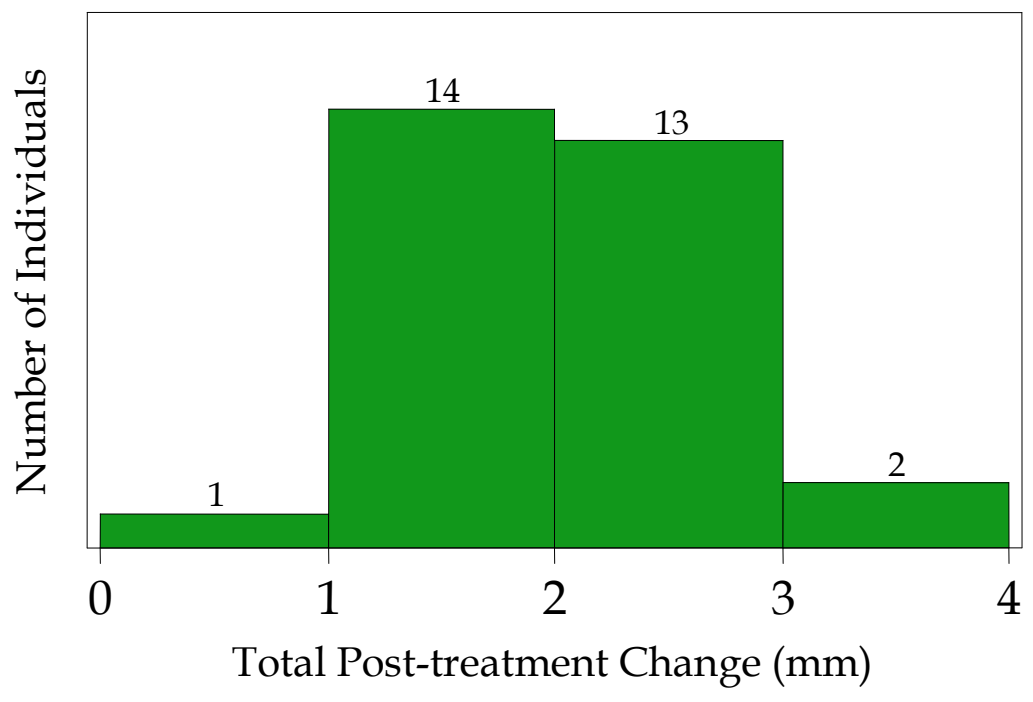

Figure C-55. Plot of the distribution of posttreatment changes in Nasion Perpendicular-Pronasale distance among the 30 women in the sample. Numbers atop each bar are the numbers of cases in that interval.

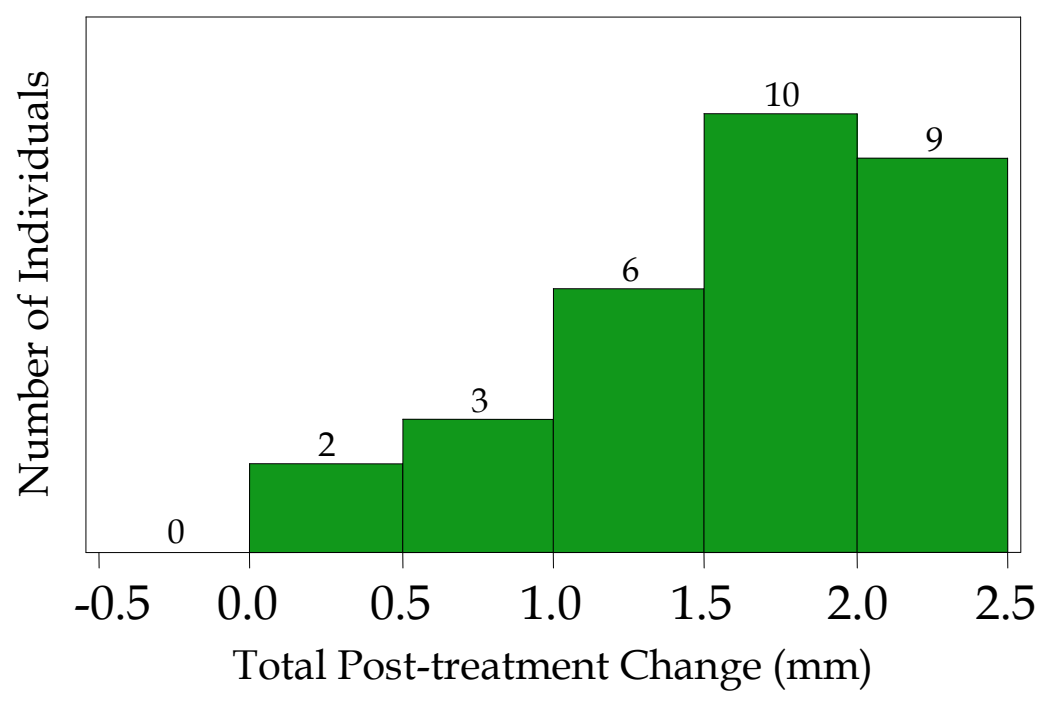

Figure C-56. Plot of the distribution of posttreatment changes in W PointPogonion distance among the 30 women in the sample. Numbers atop each bar are the numbers of cases in that interval. 
APPENDIX D.

GRAPHICAL REPRESENTATIONS OF CEPHALOMETRIC VARIABLES 


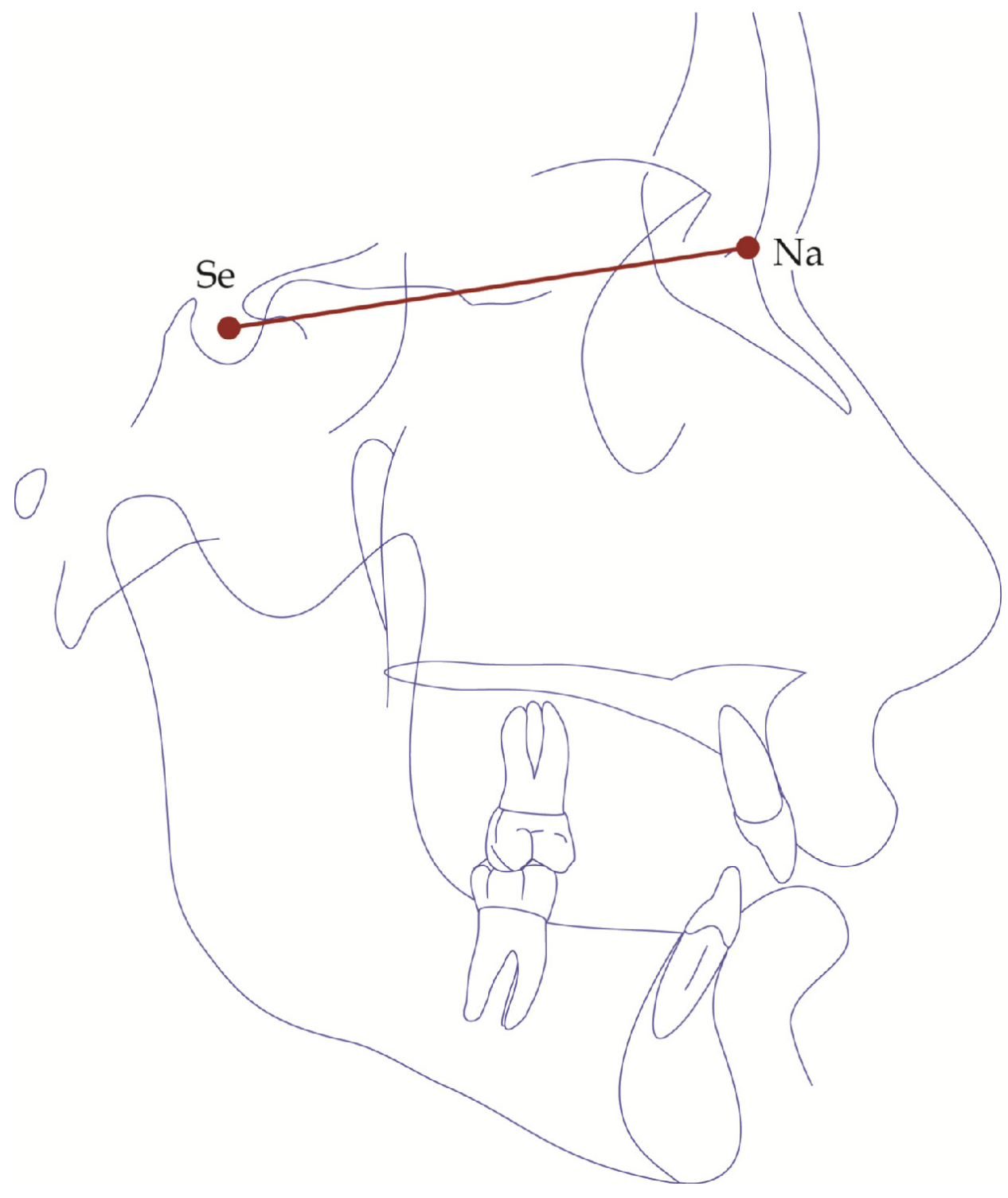

Figure D-1. Schematic tracing of a lateral cephalogram showing construction of Se-Na (mm). 


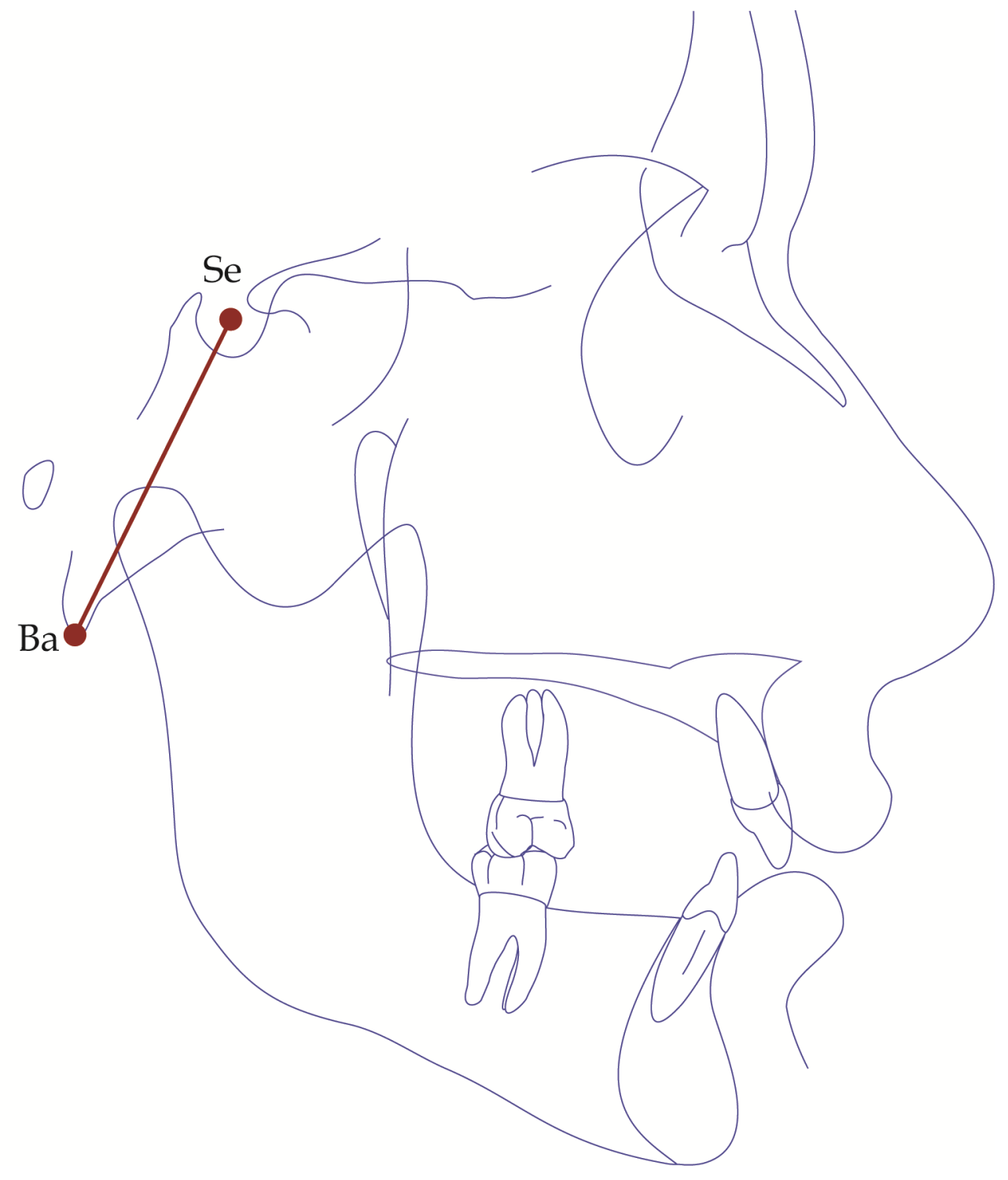

Figure D-2. Schematic tracing of a lateral cephalogram showing construction of Se-Ba (mm). 


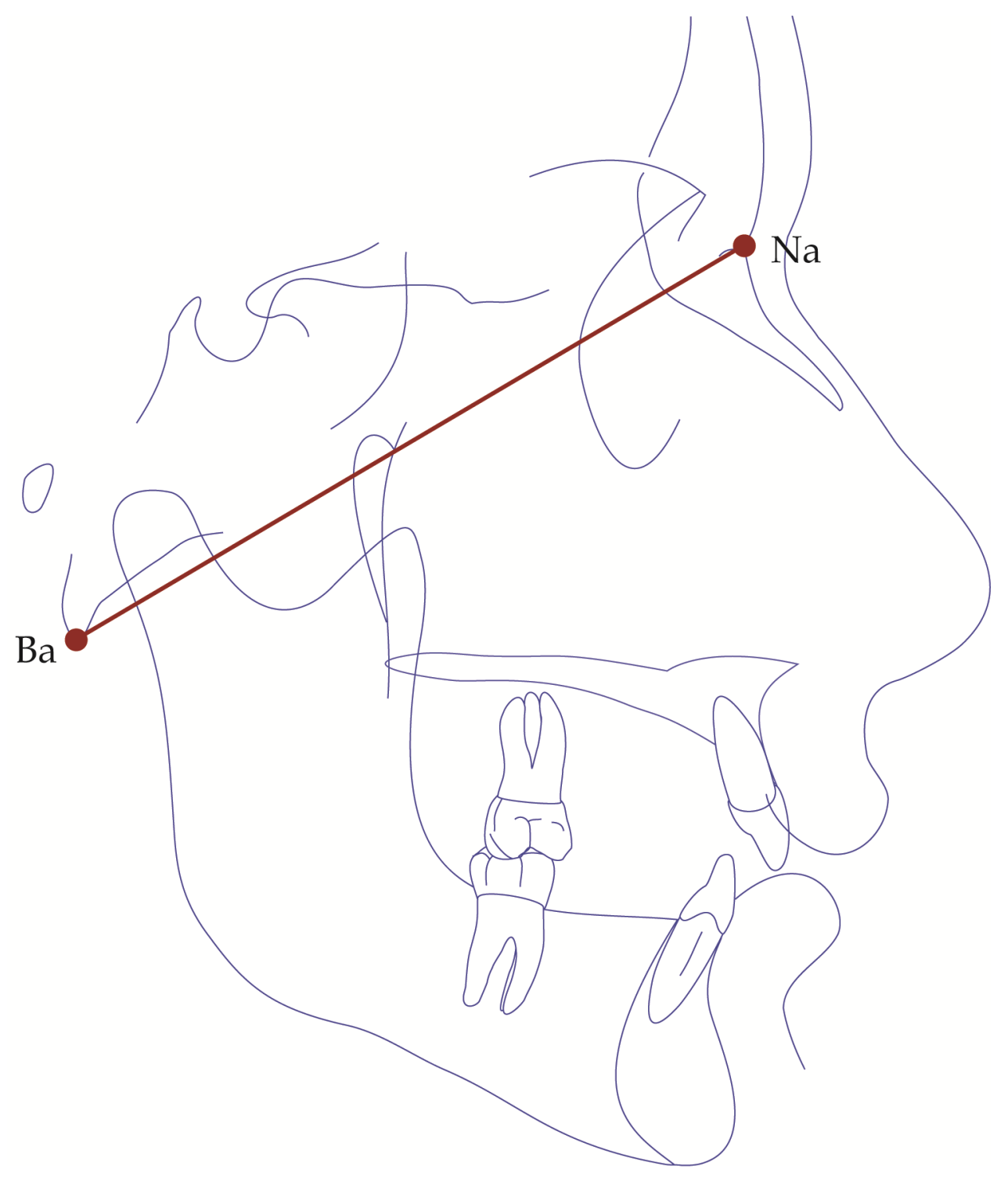

Figure D-3. Schematic tracing of a lateral cephalogram showing construction of $\mathrm{Na}-\mathrm{Ba}(\mathrm{mm})$. 


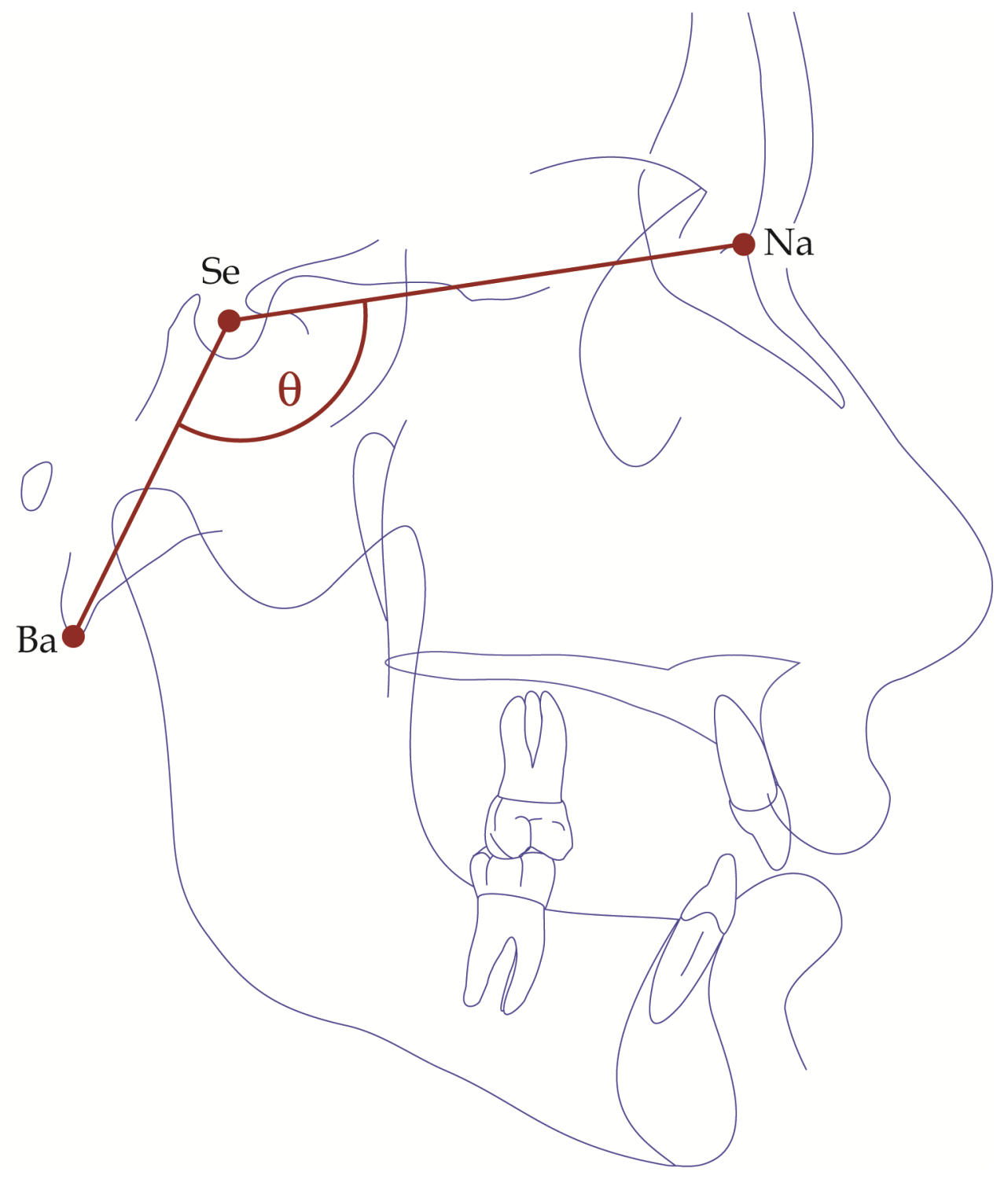

Figure D-4. Schematic tracing of a lateral cephalogram showing construction of Na-Se-Ba angle. 


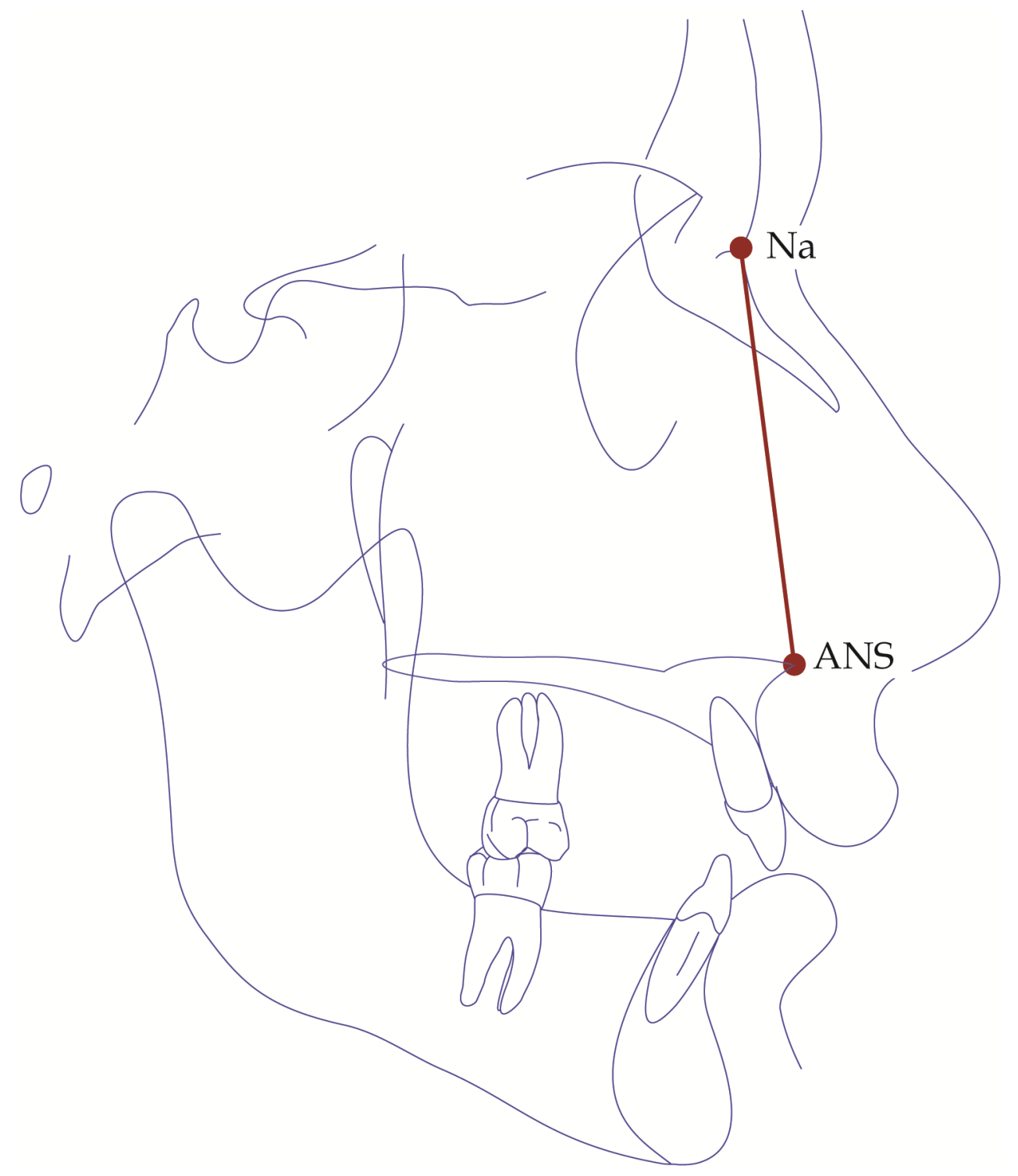

Figure D-5. Schematic tracing of a lateral cephalogram showing construction of Na-ANS (mm). 


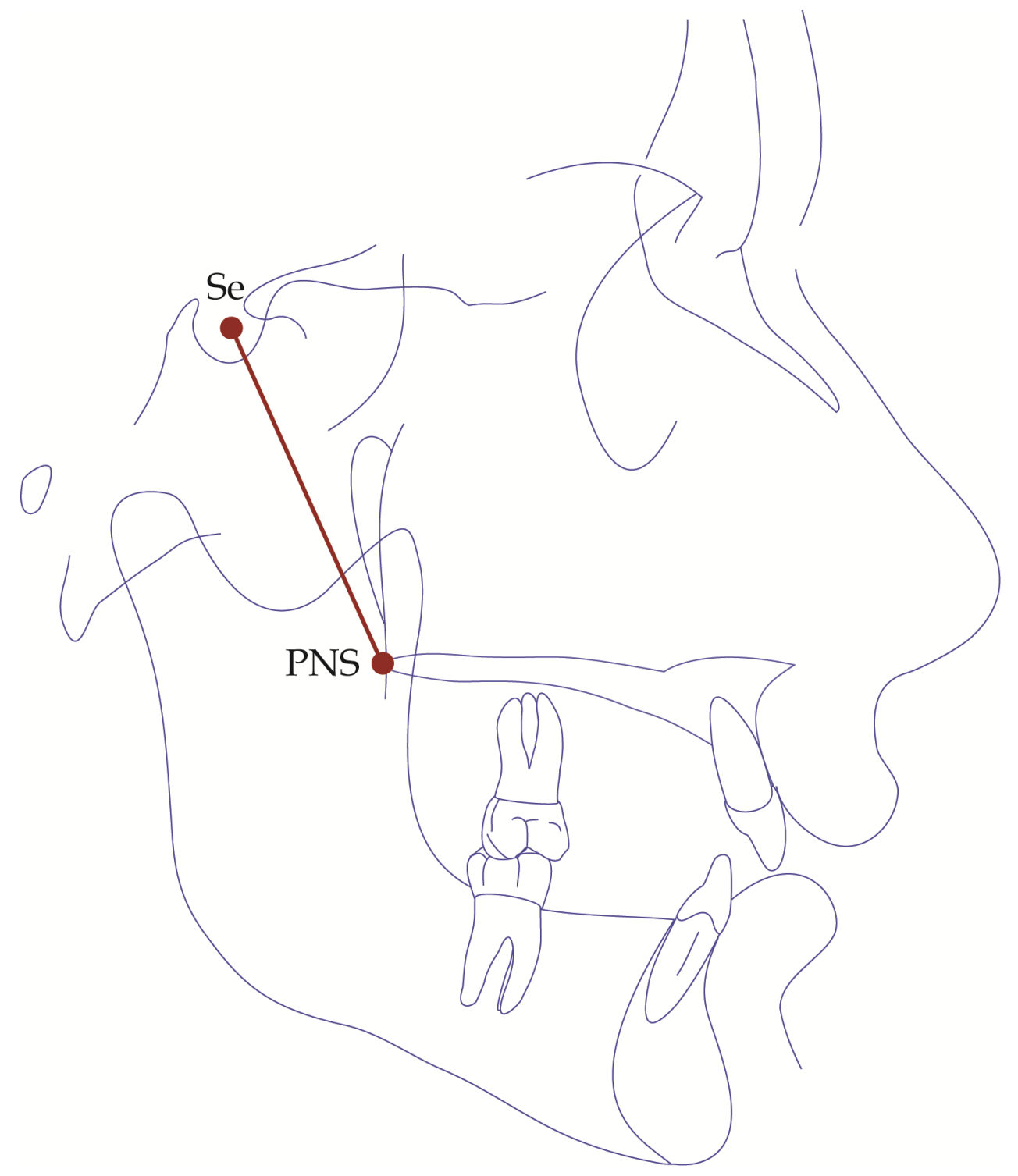

Figure D-6. Schematic tracing of a lateral cephalogram showing construction of Se-PNS (mm). 


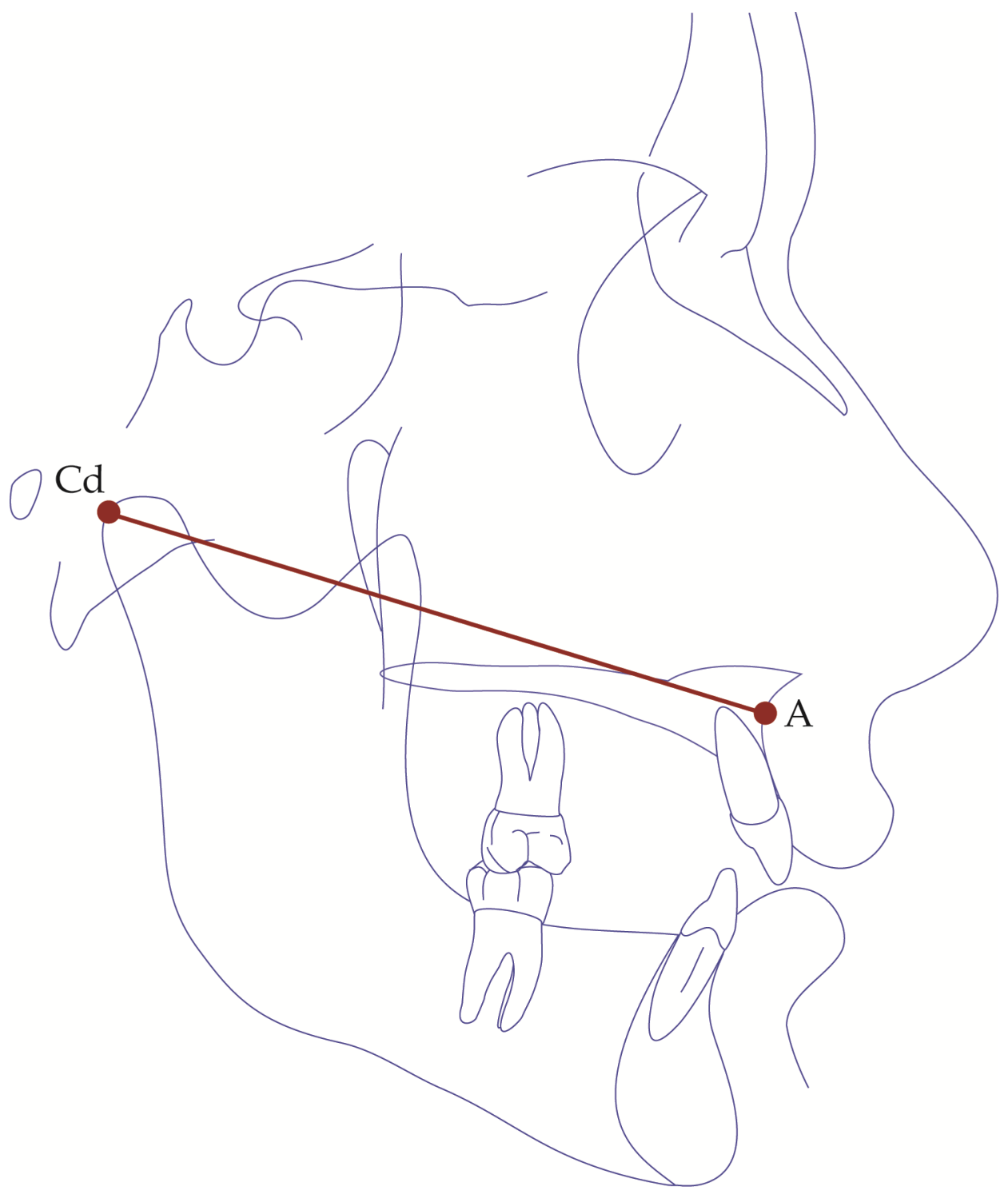

Figure D-7. Schematic tracing of a lateral cephalogram showing construction of Cd-A (mm). 


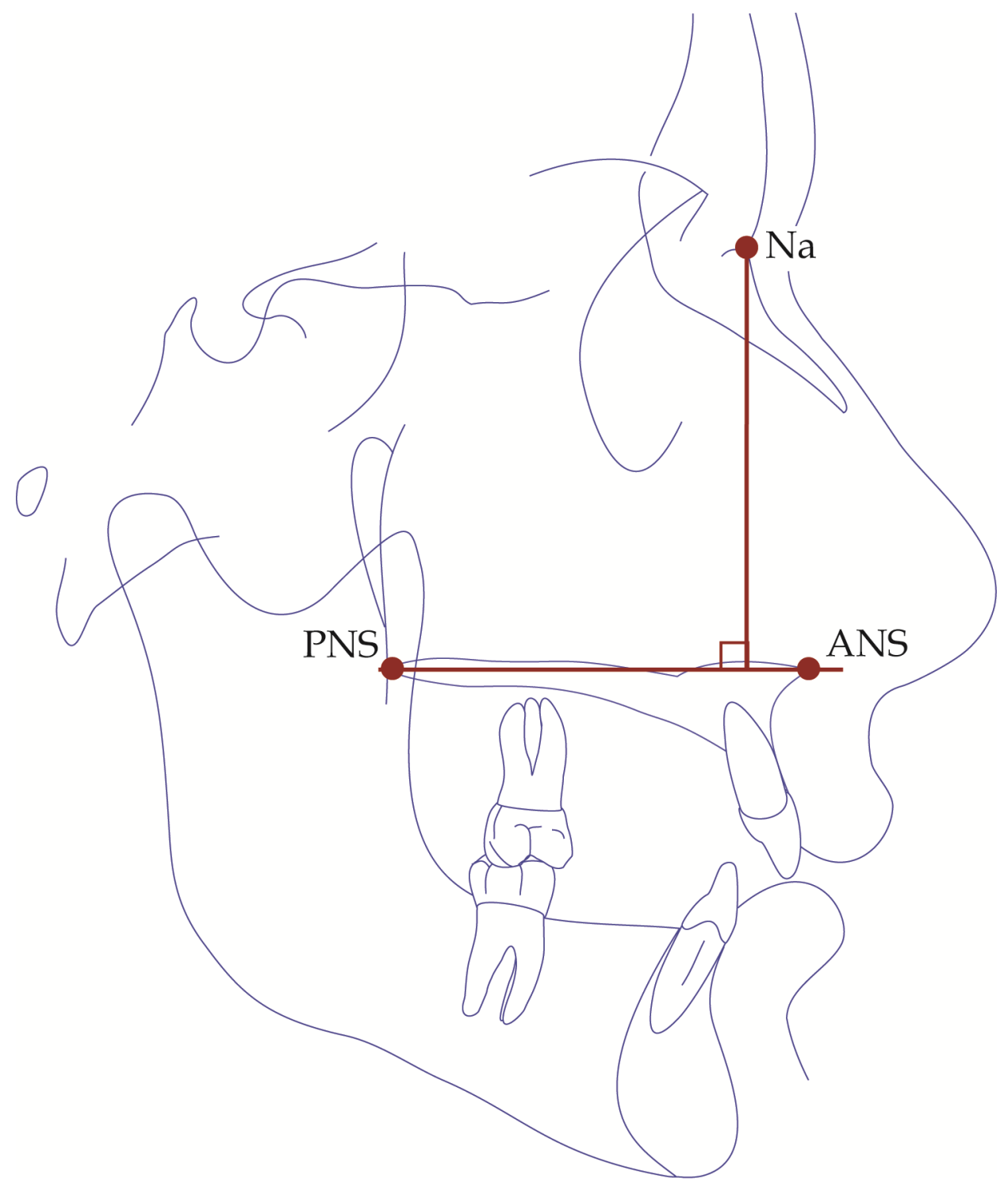

Figure D-8. Schematic tracing of a lateral cephalogram showing construction of $\mathrm{Na}-\mathrm{PP}(\mathrm{mm})$. 


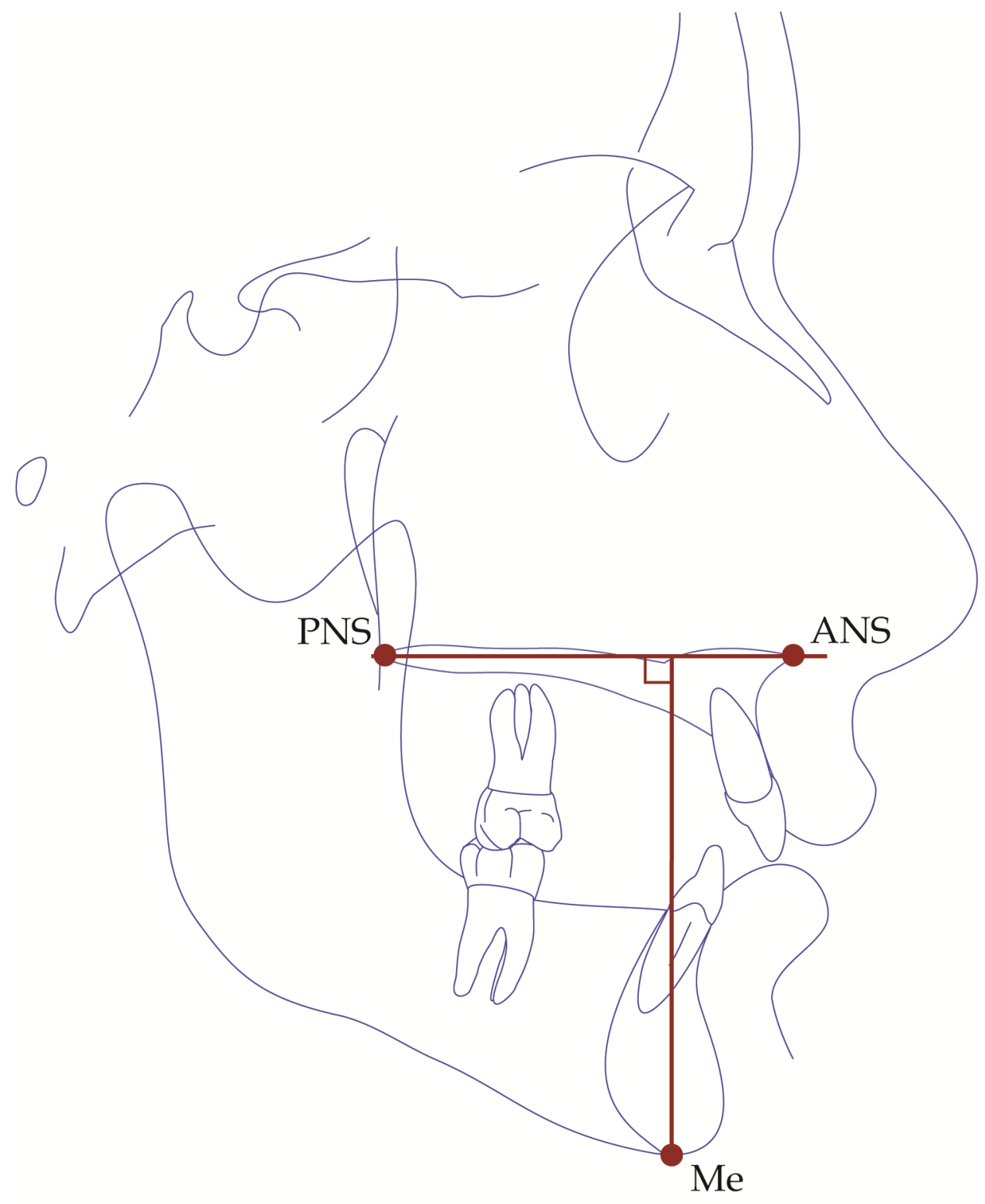

Figure D-9. Schematic tracing of a lateral cephalogram showing construction of Me-PP (mm). 


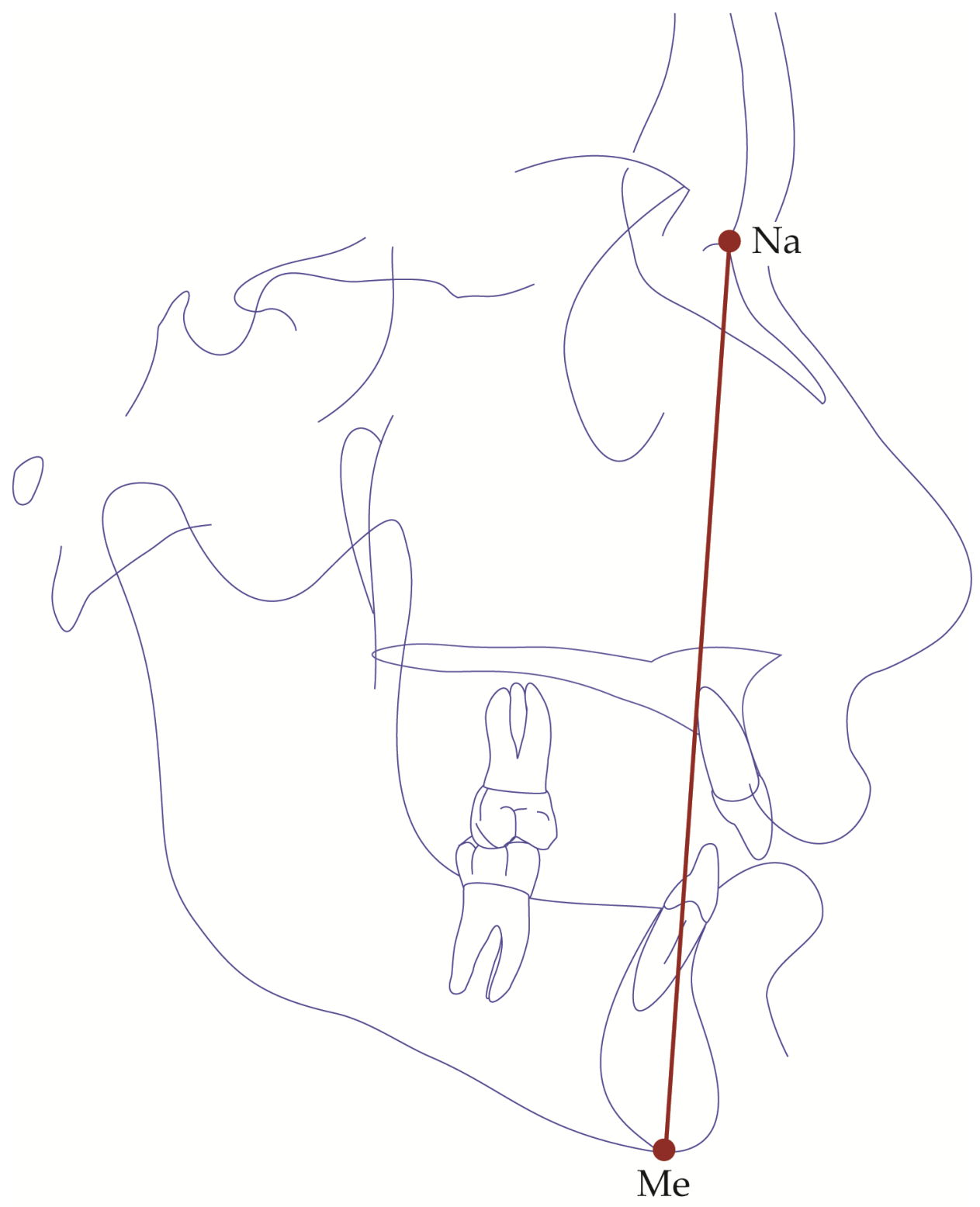

Figure D-10. Schematic tracing of a lateral cephalogram showing construction of $\mathrm{Na}-\mathrm{Me}(\mathrm{mm})$. 


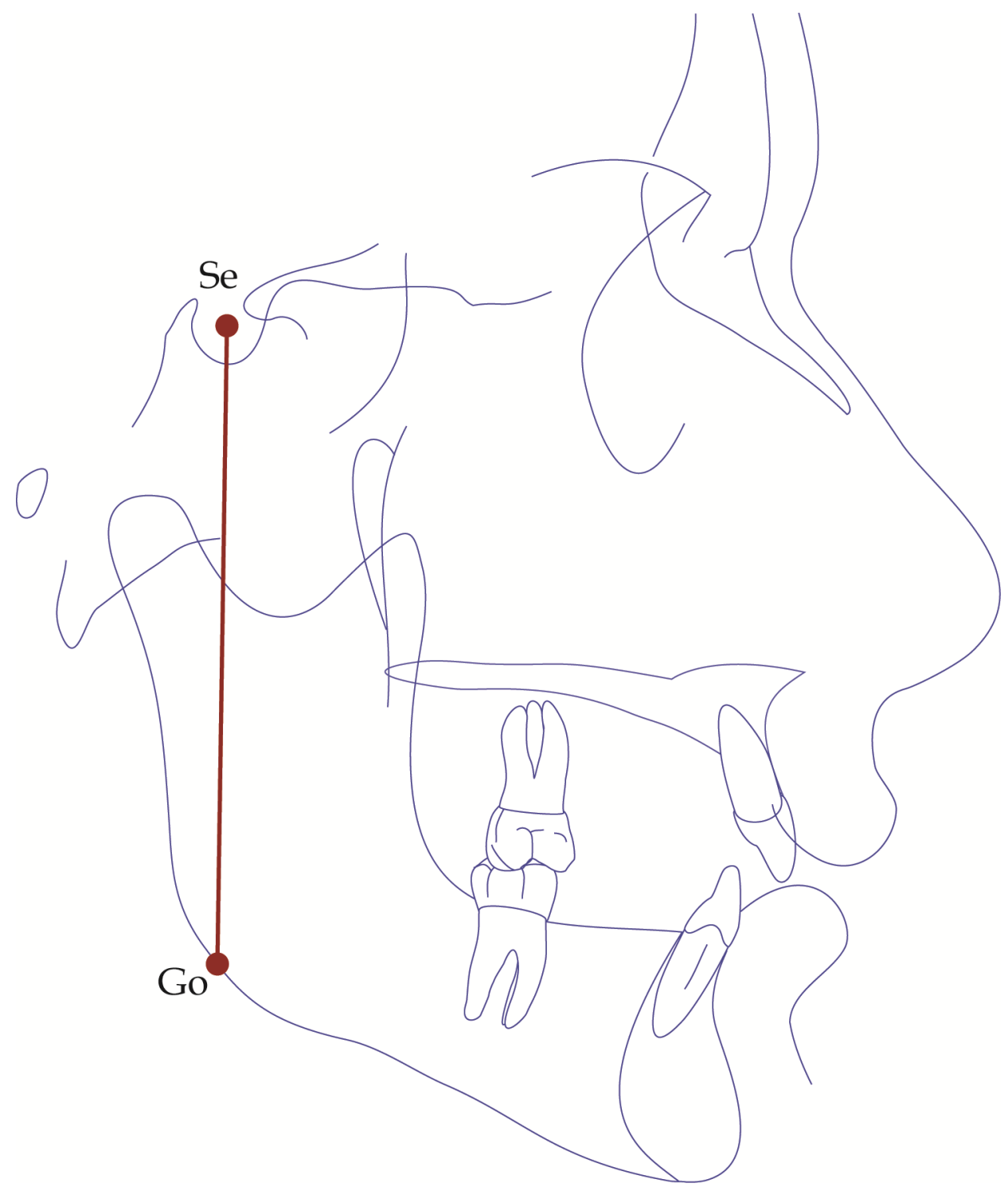

Figure D-11. Schematic tracing of a lateral cephalogram showing construction of Se-Go (mm). 


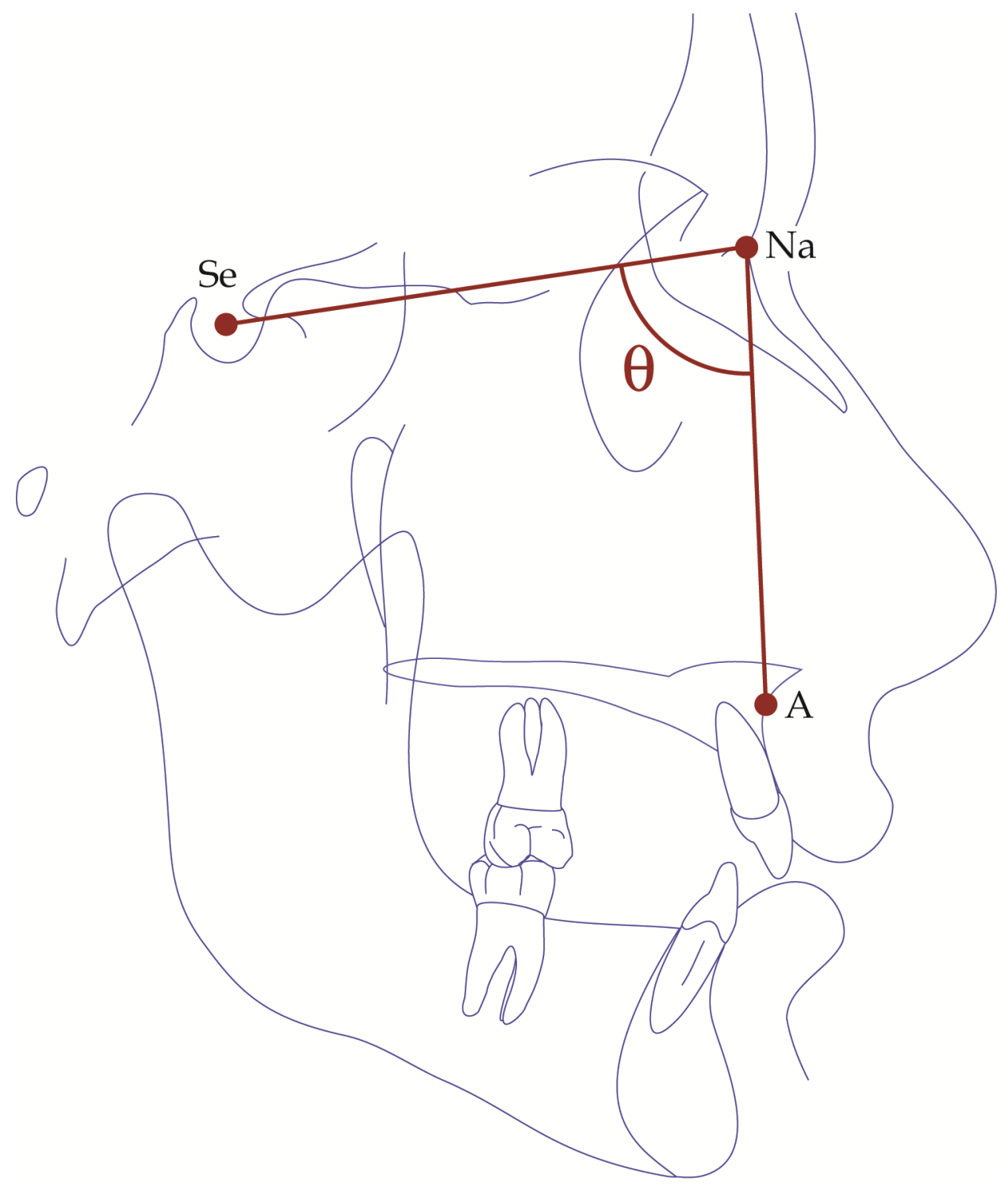

Figure D-12. Schematic tracing of a lateral cephalogram showing construction of SNA angle. 


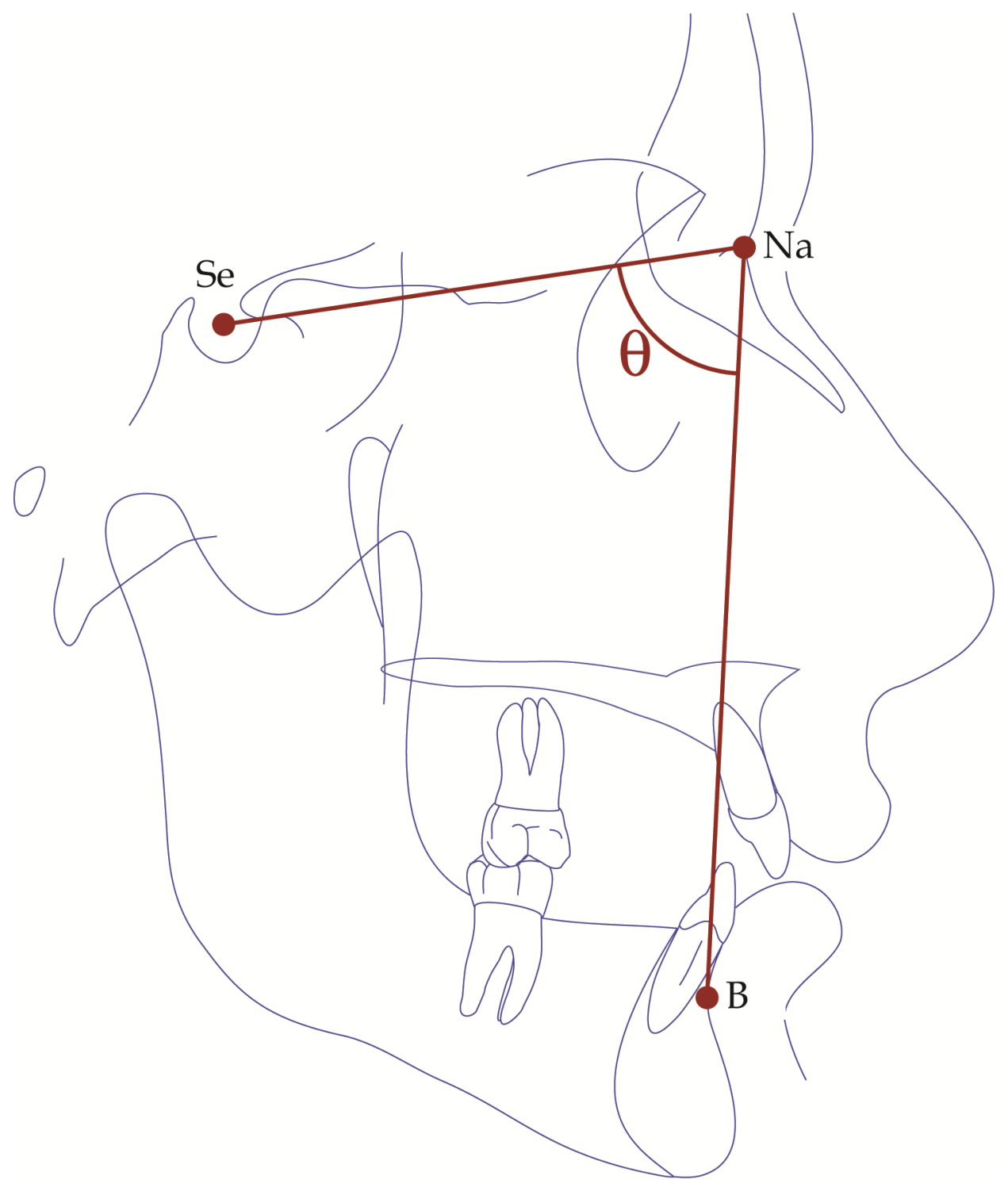

Figure D-13. Schematic tracing of a lateral cephalogram showing construction of SNB angle. 


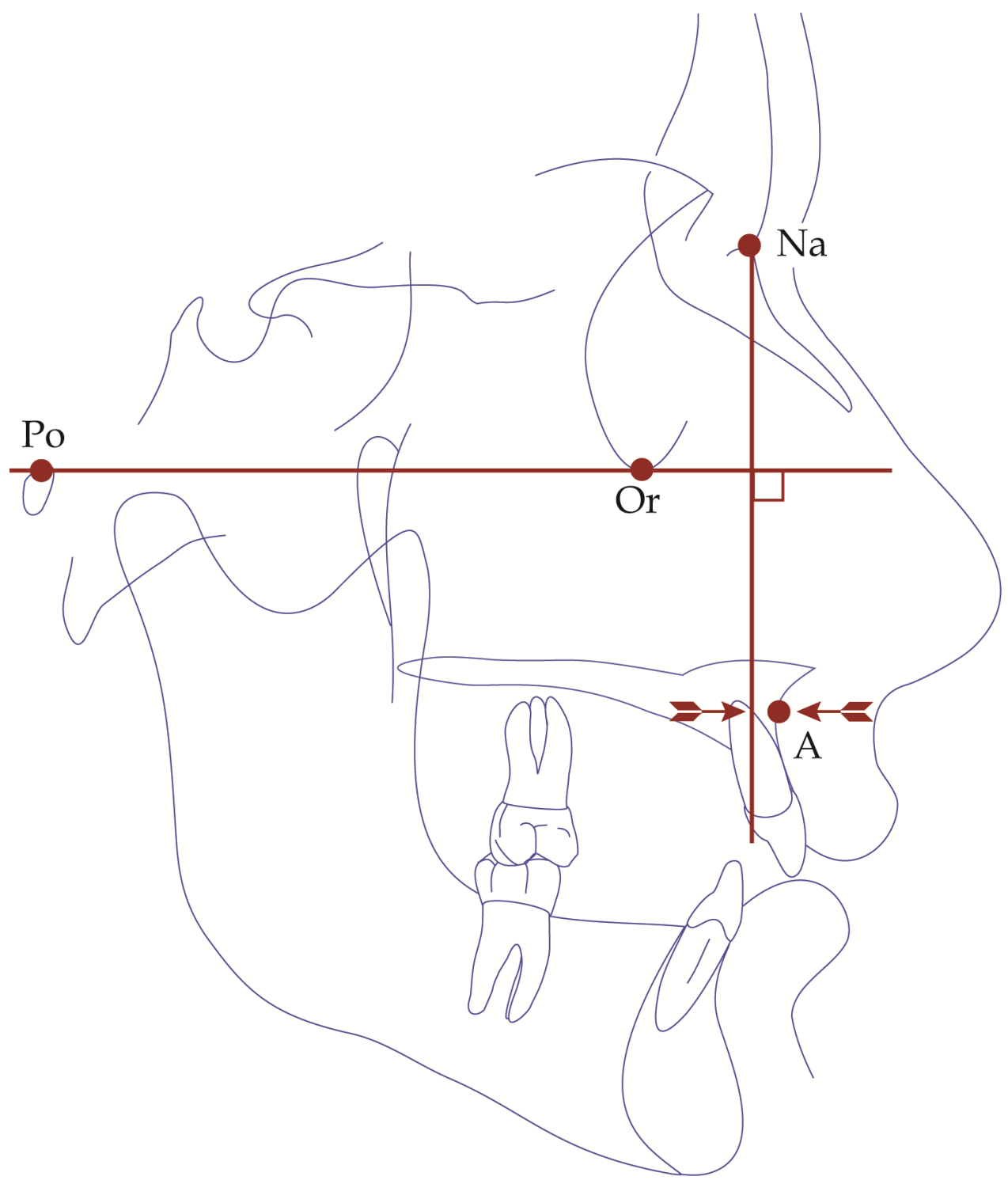

Figure D-14. Schematic tracing of a lateral cephalogram showing construction of NaPerp-A (mm). 


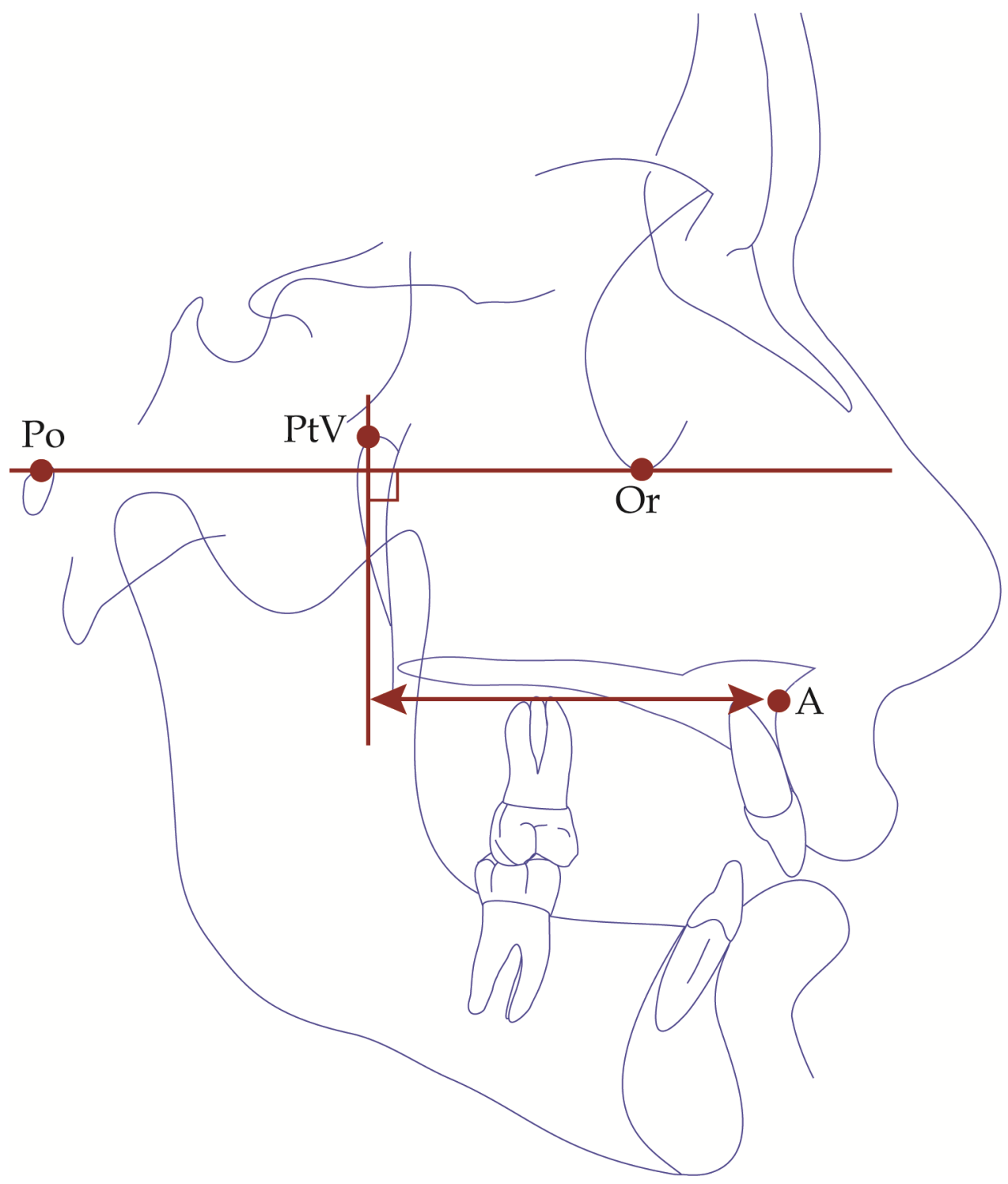

Figure D-15. Schematic tracing of a lateral cephalogram showing construction of PtV-A (mm). 


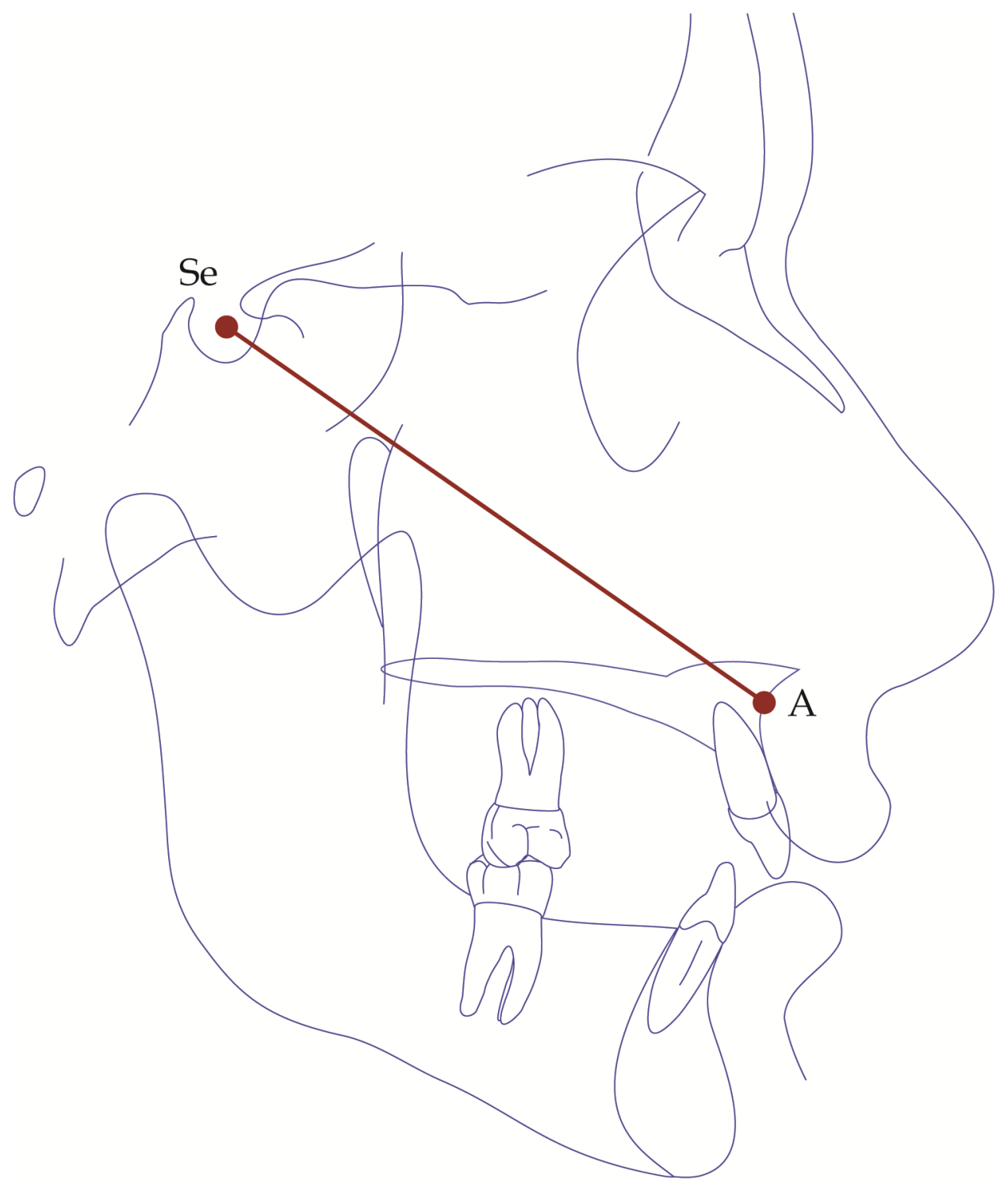

Figure D-16. Schematic tracing of a lateral cephalogram showing construction of Se-A (mm). 


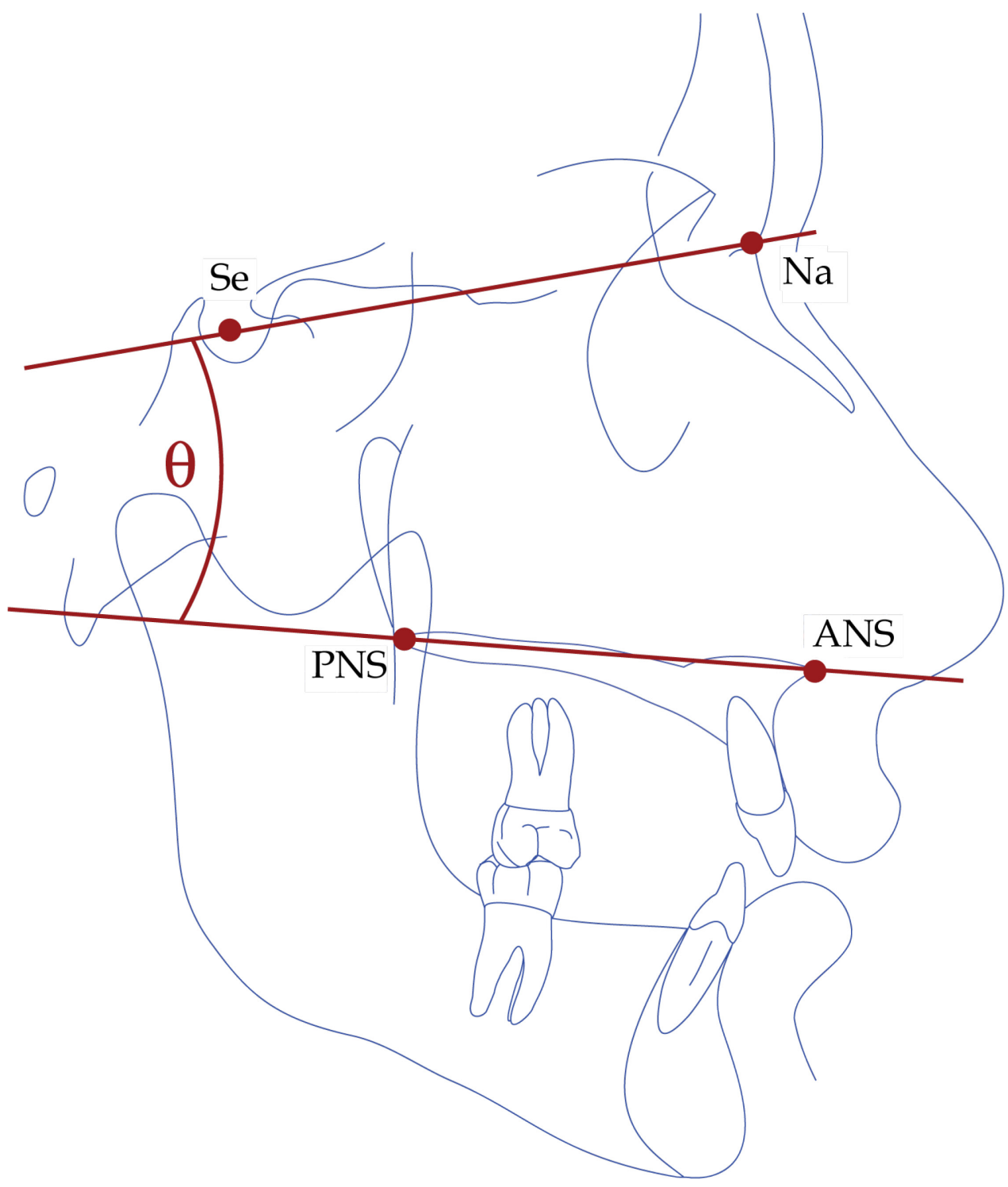

Figure D-17. Schematic tracing of a lateral cephalogram showing construction of PP-SN angle. 


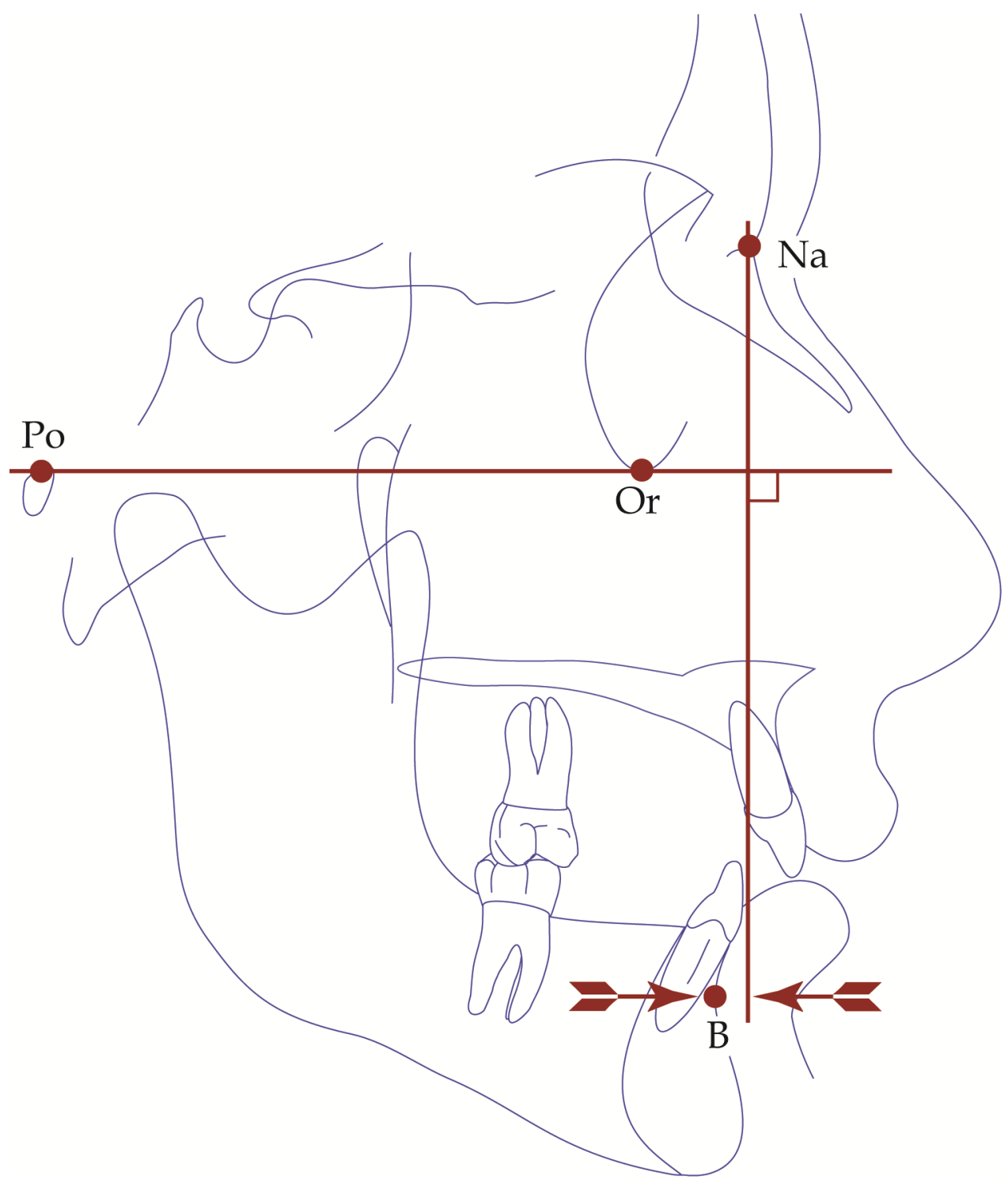

Figure D-18. Schematic tracing of a lateral cephalogram showing construction of NaPerp-B (mm). 


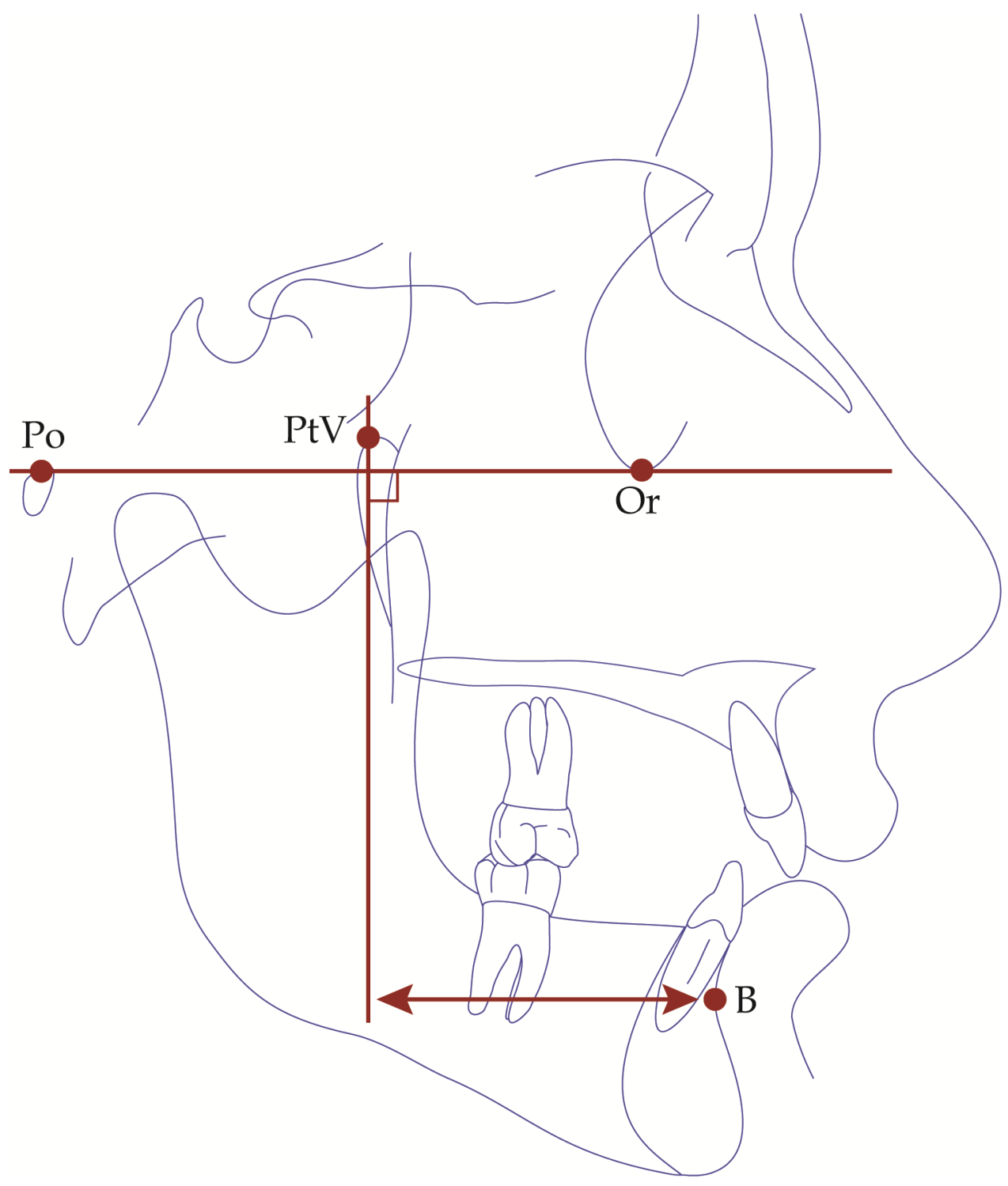

Figure D-19. Schematic tracing of a lateral cephalogram showing construction of $\mathrm{PtV}-\mathrm{B}(\mathrm{mm})$. 


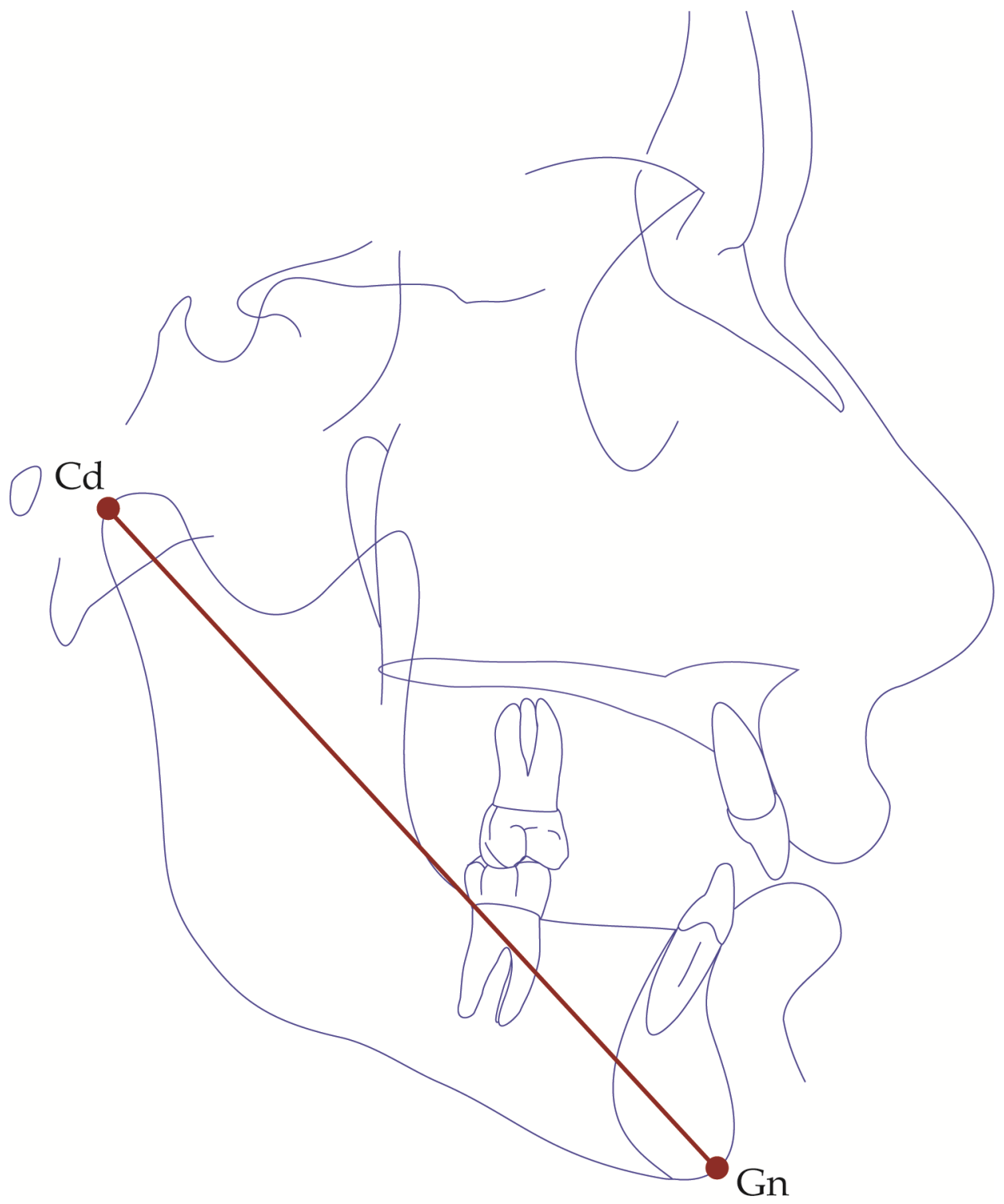

Figure D-20. Schematic tracing of a lateral cephalogram showing construction of Cd-Gn (mm). 


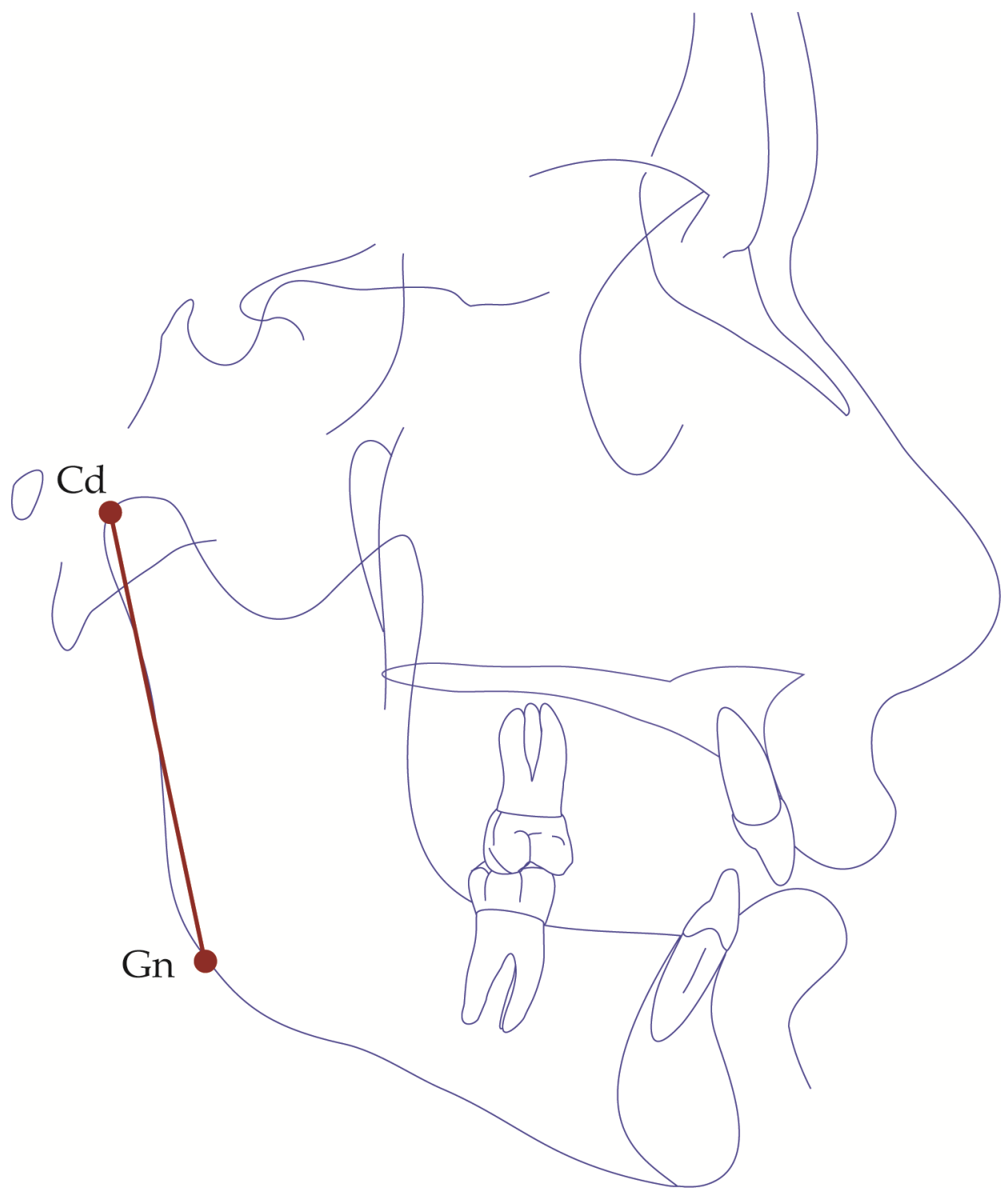

Figure D-21. Schematic tracing of a lateral cephalogram showing construction of Cd-Go (mm). 


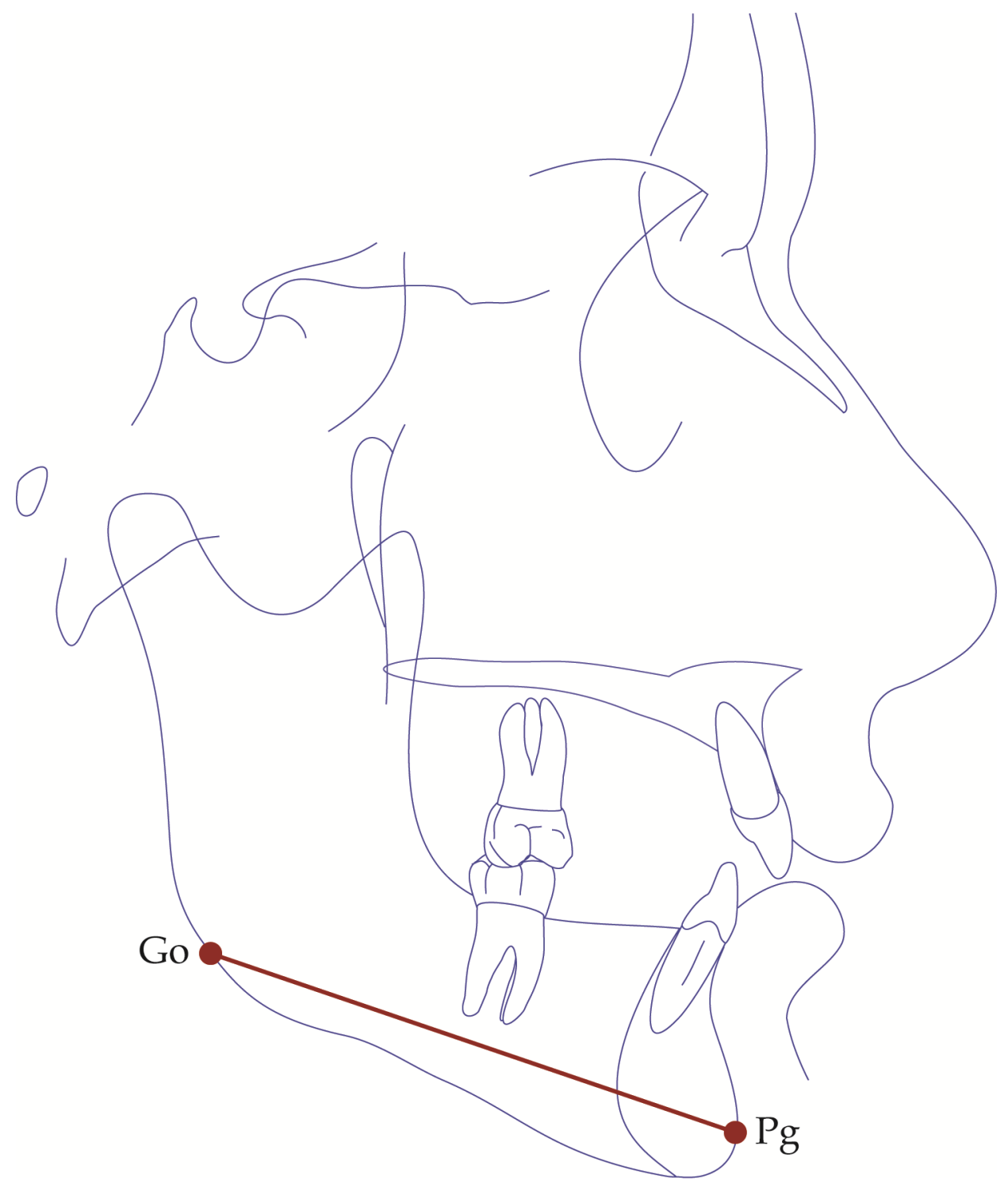

Figure D-22. Schematic tracing of a lateral cephalogram showing construction of Go-Pg (mm). 


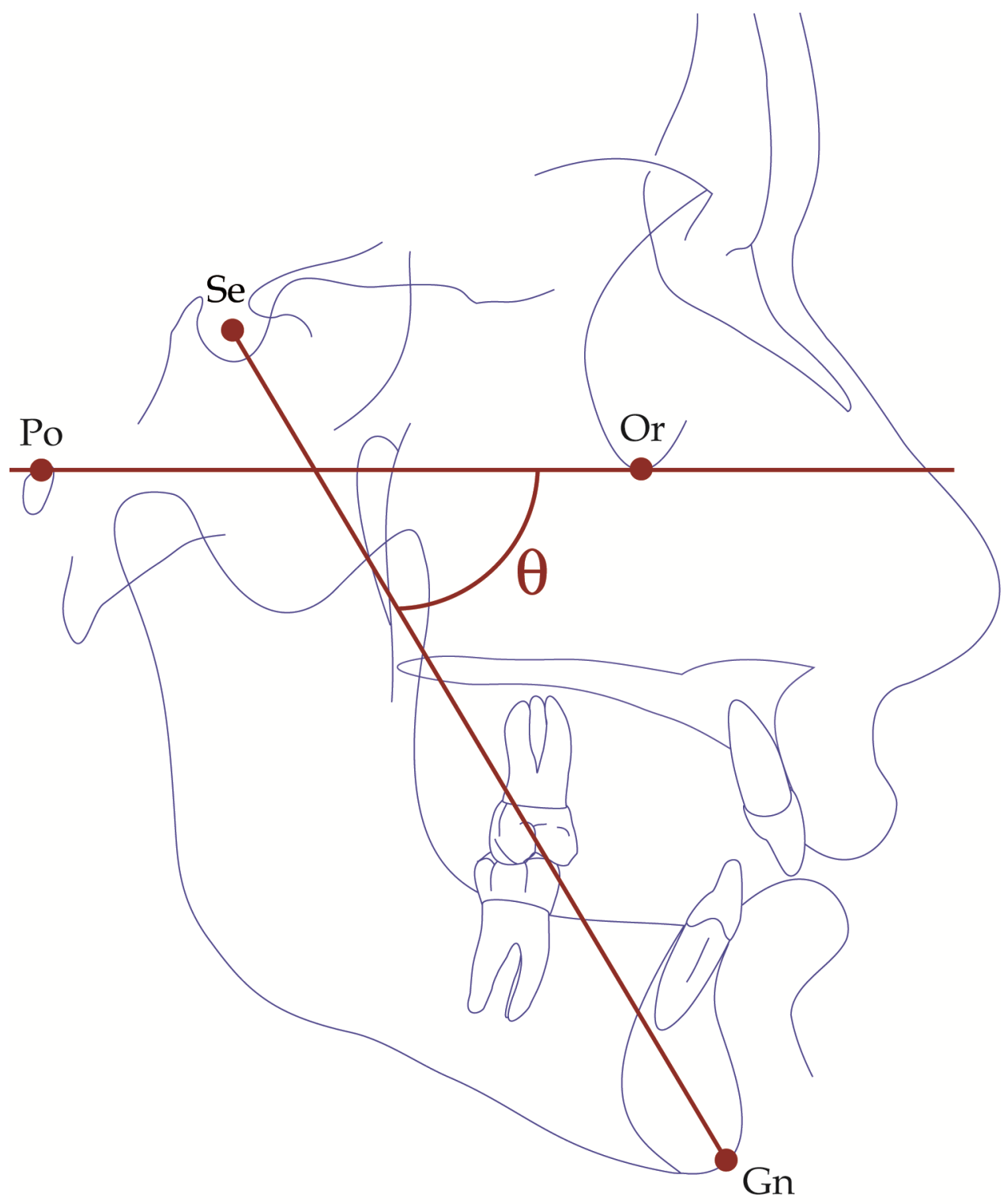

Figure D-23. Schematic tracing of a lateral cephalogram showing construction of Y-Axis angle. 


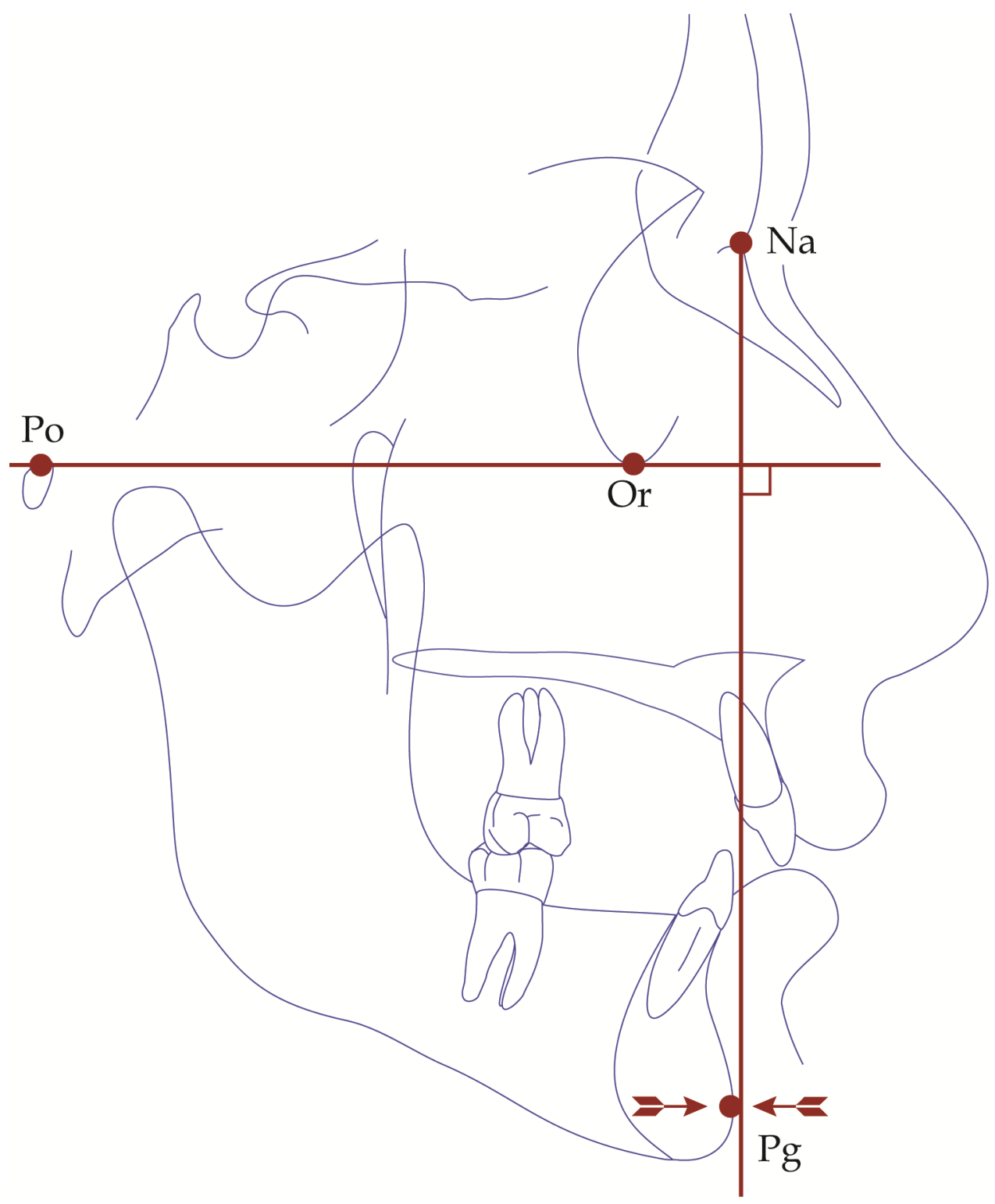

Figure D-24. Schematic tracing of a lateral cephalogram showing construction of NaPerp-Pg (mm). 


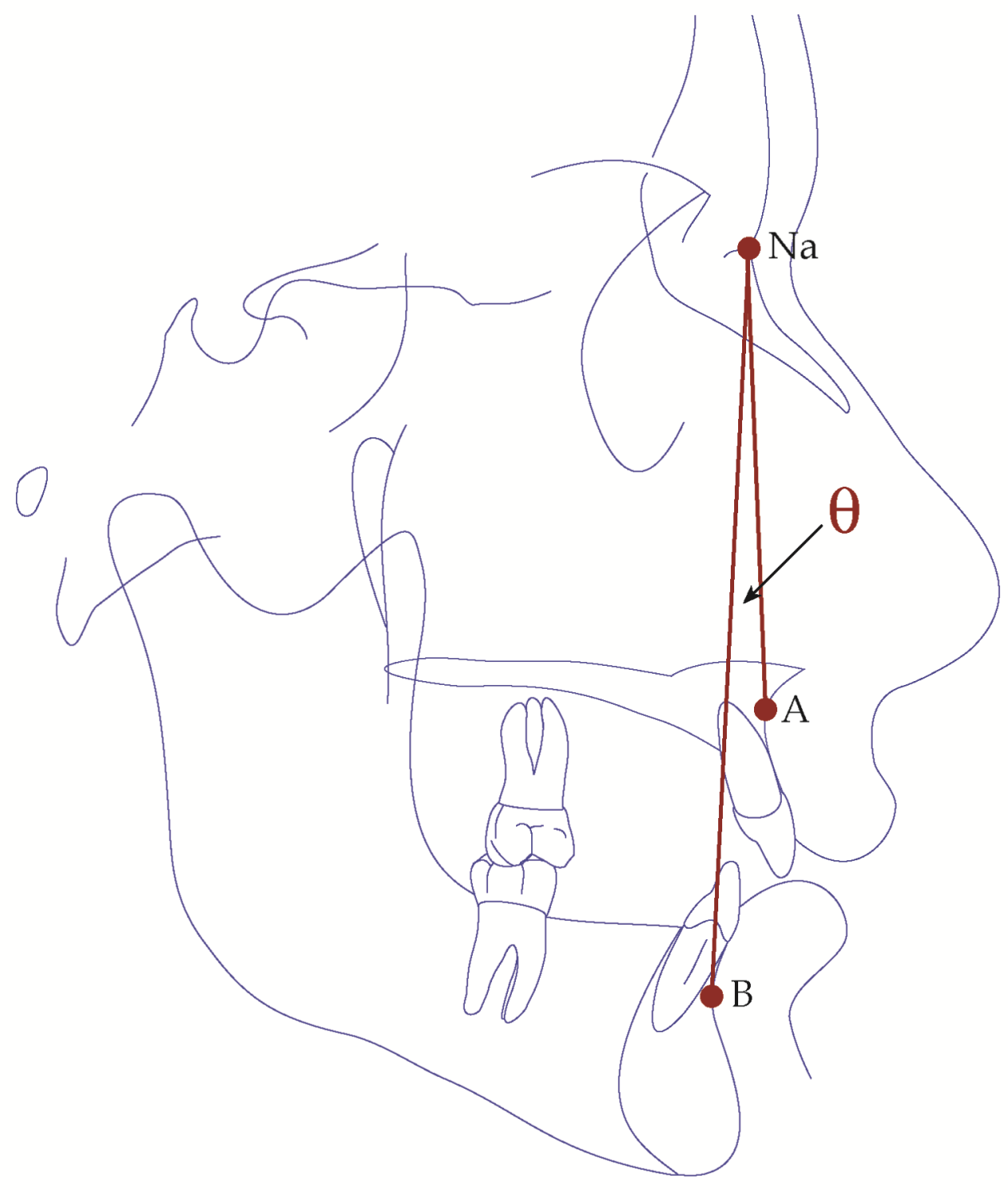

Figure D-25. Schematic tracing of a lateral cephalogram showing construction of ANB angle. 


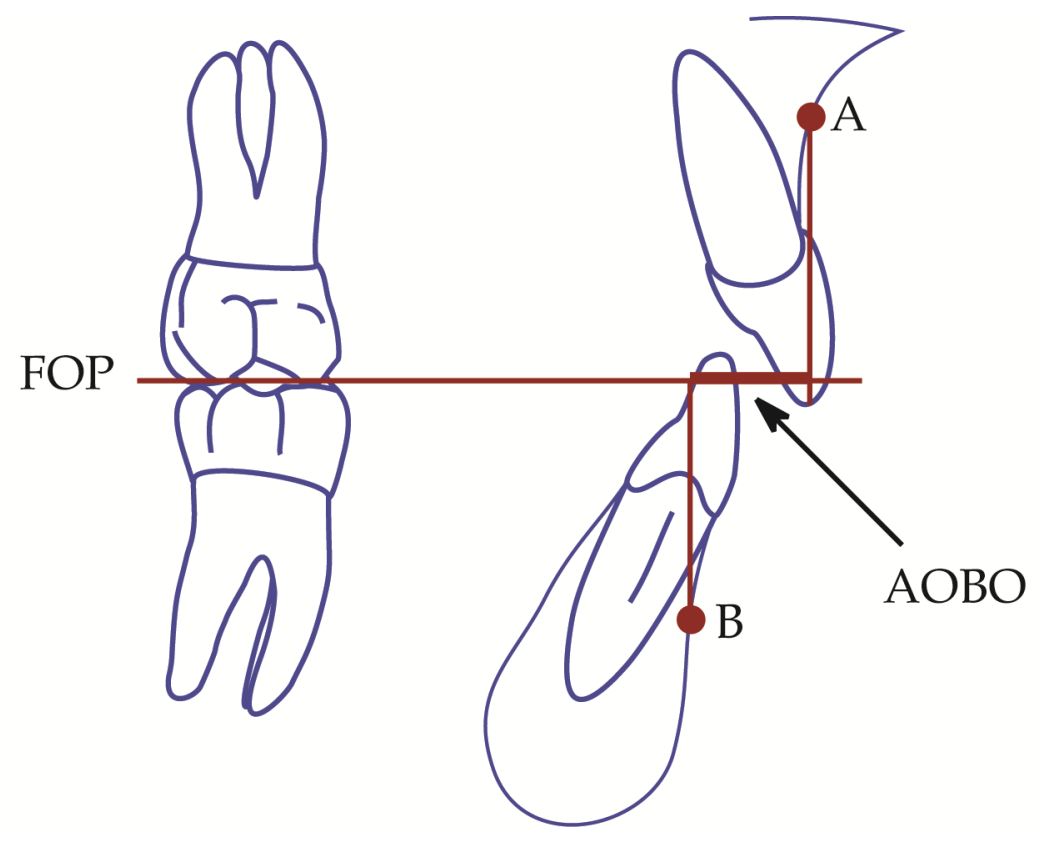

Figure D-26. Schematic tracing of a lateral cephalogram showing construction of $\mathrm{AO}-\mathrm{BO}(\mathrm{mm})$. 


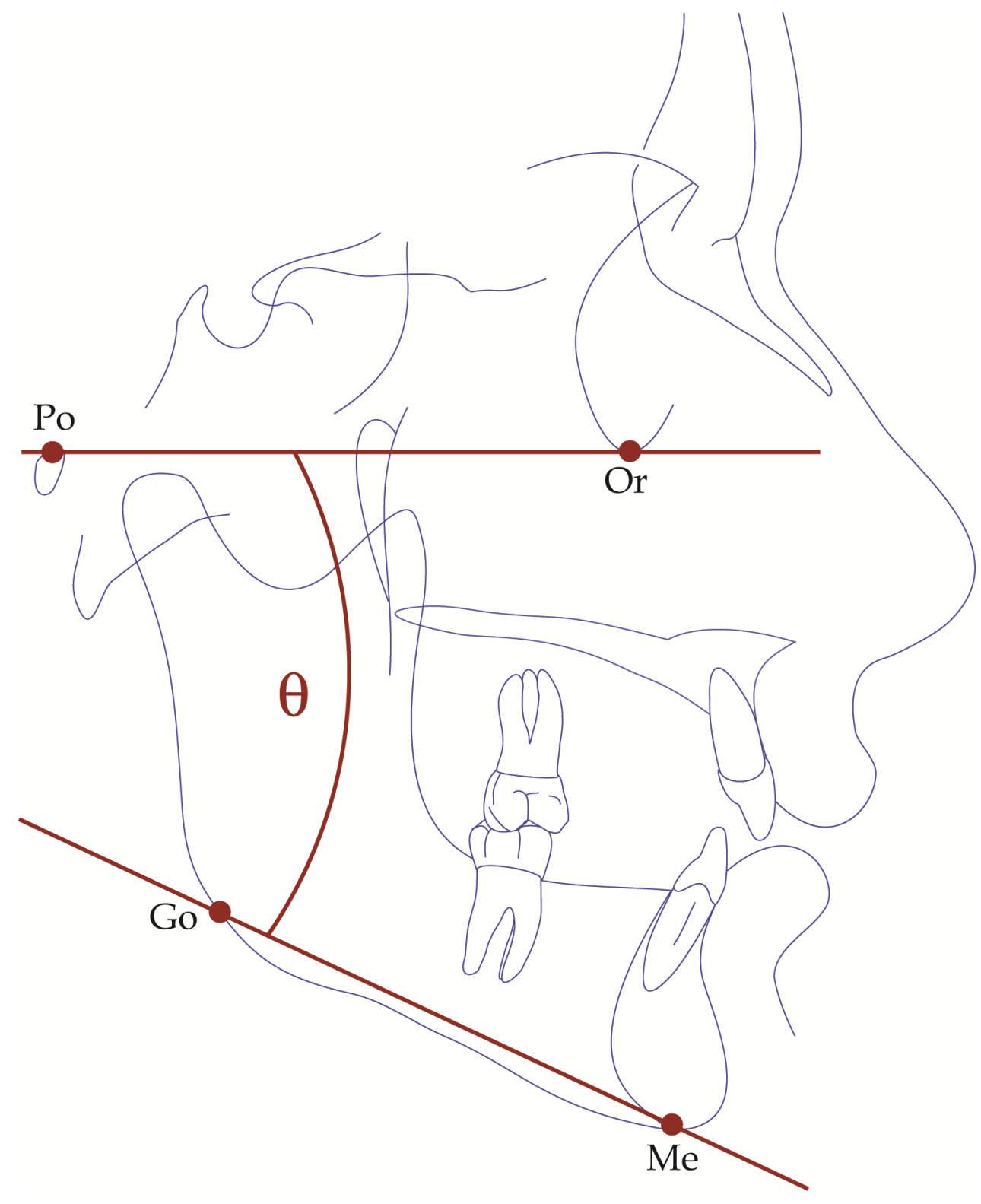

Figure D-27. Schematic tracing of a lateral cephalogram showing construction of FMA angle. 


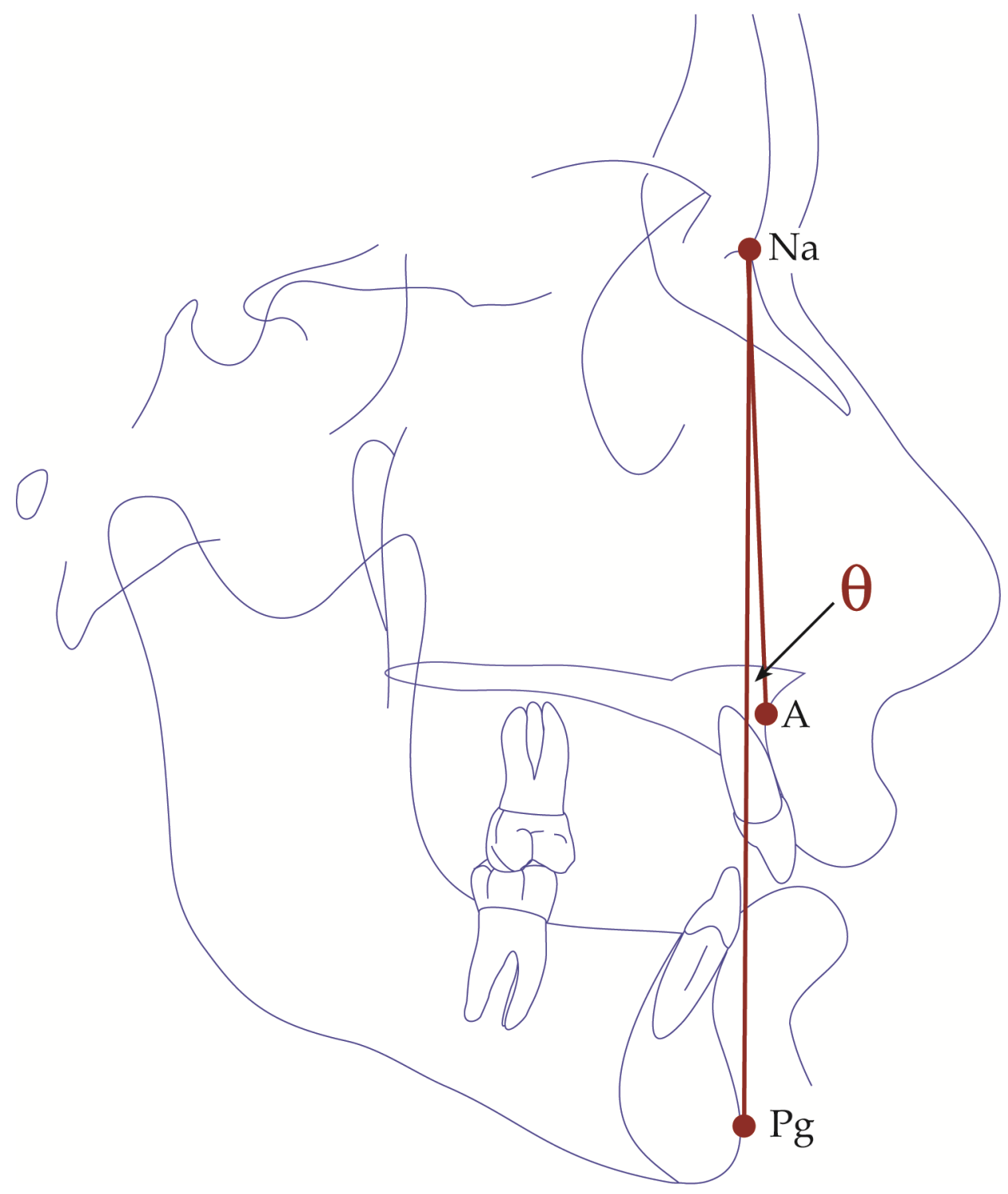

Figure D-28. Schematic tracing of a lateral cephalogram showing construction of Na-A-Pg angle. 


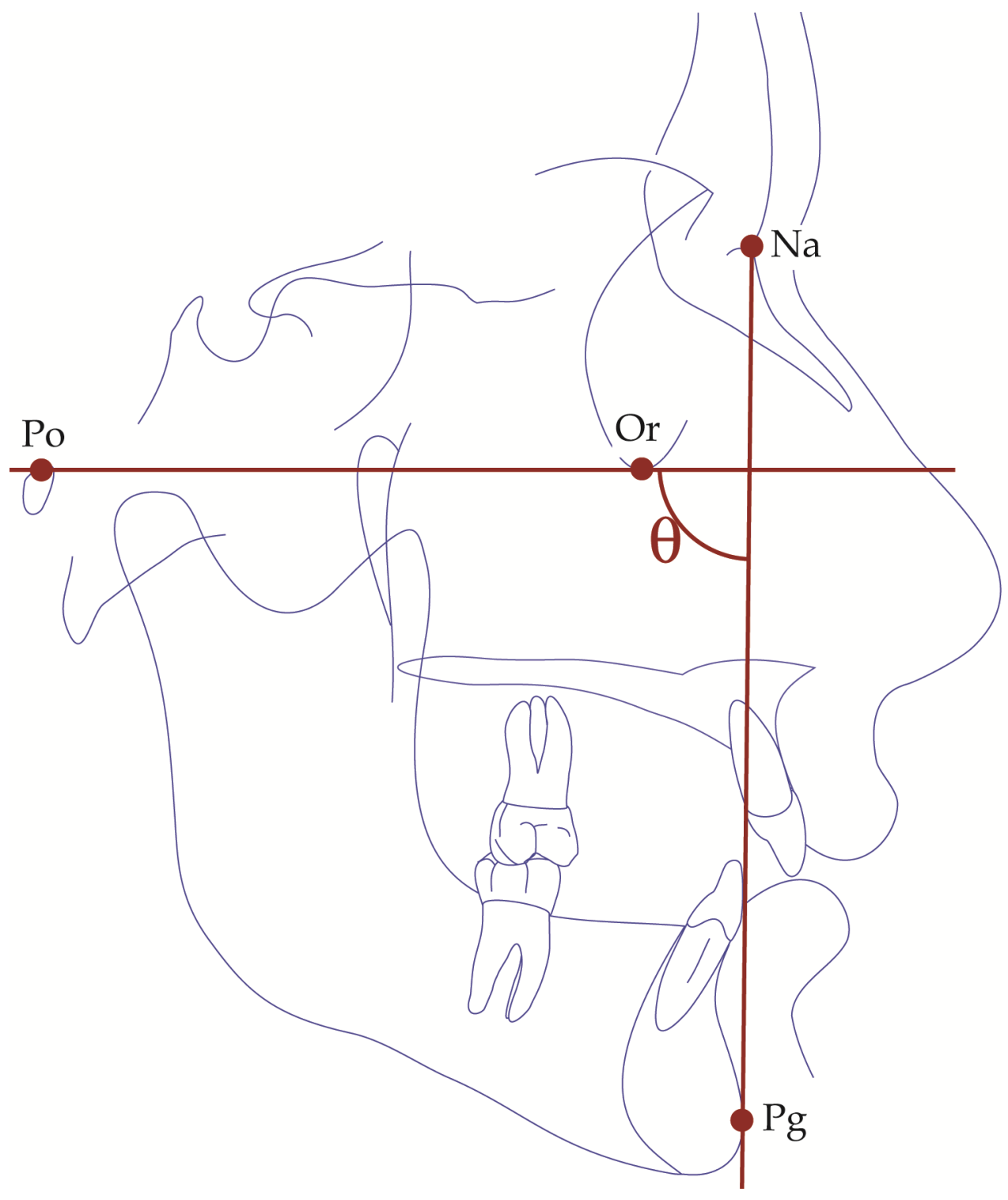

Figure D-29. Schematic tracing of a lateral cephalogram showing construction of FH-Na-Pg angle. 


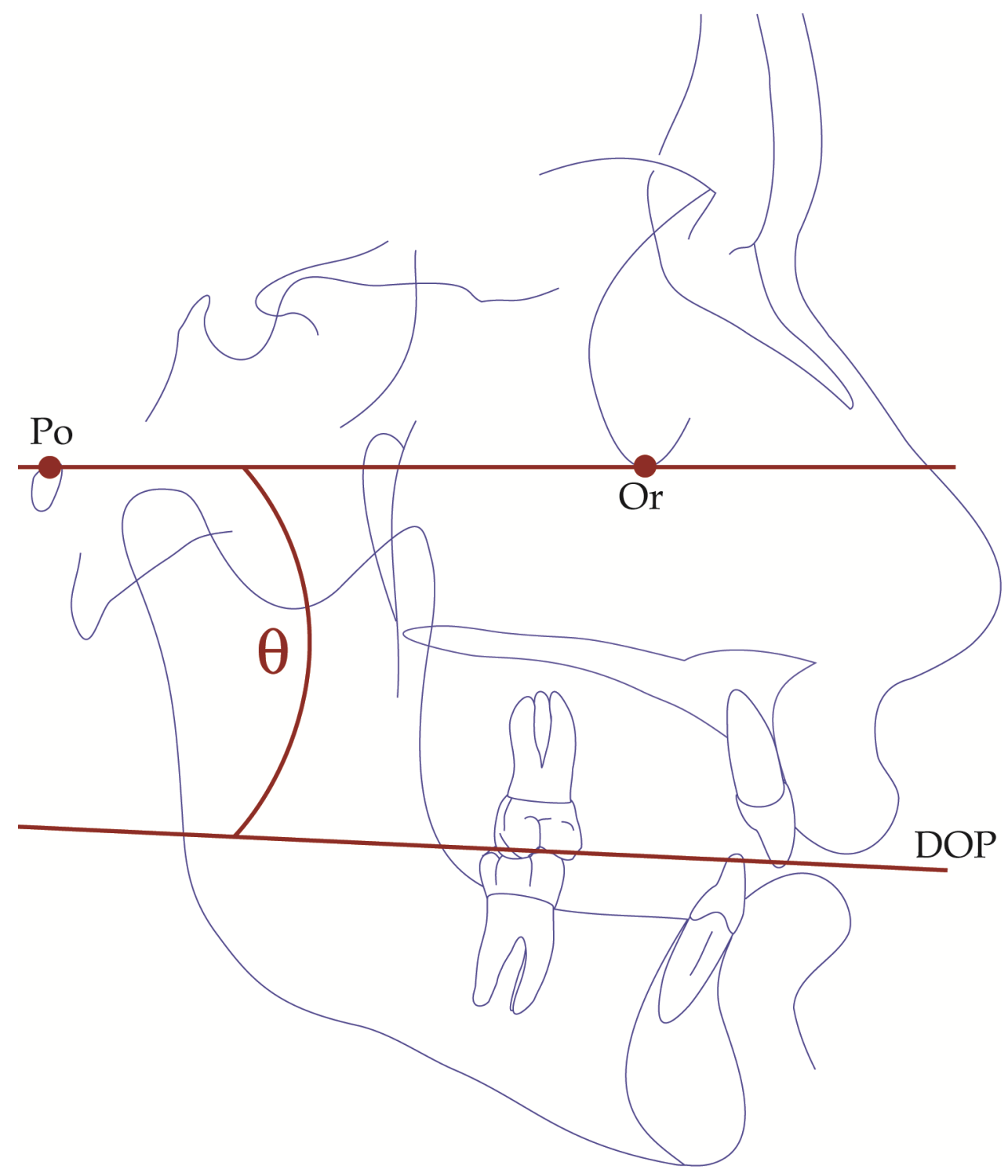

Figure D-30. Schematic tracing of a lateral cephalogram showing construction of DOP-FH angle. 


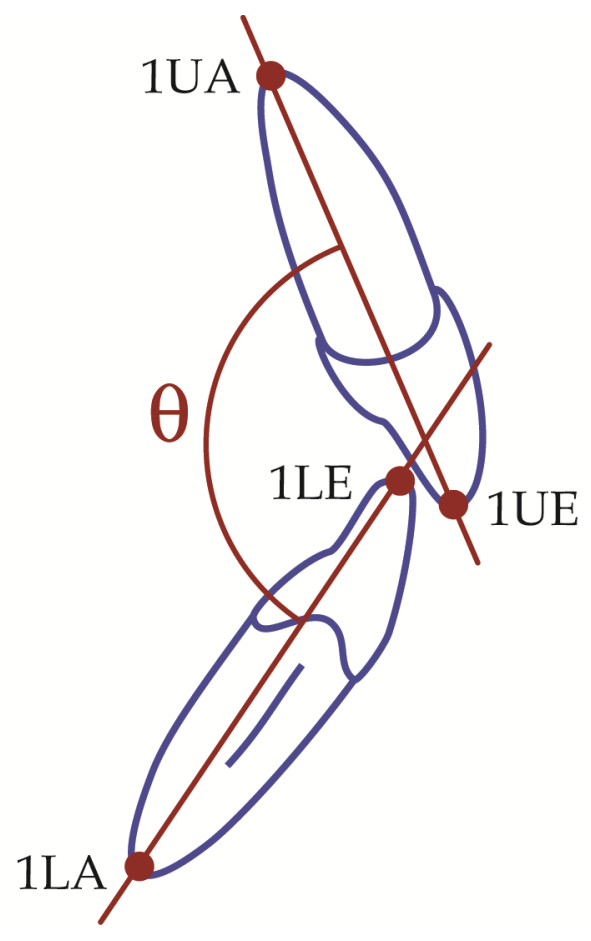

Figure D-31. Schematic tracing showing construction of U1-L1 angle. 


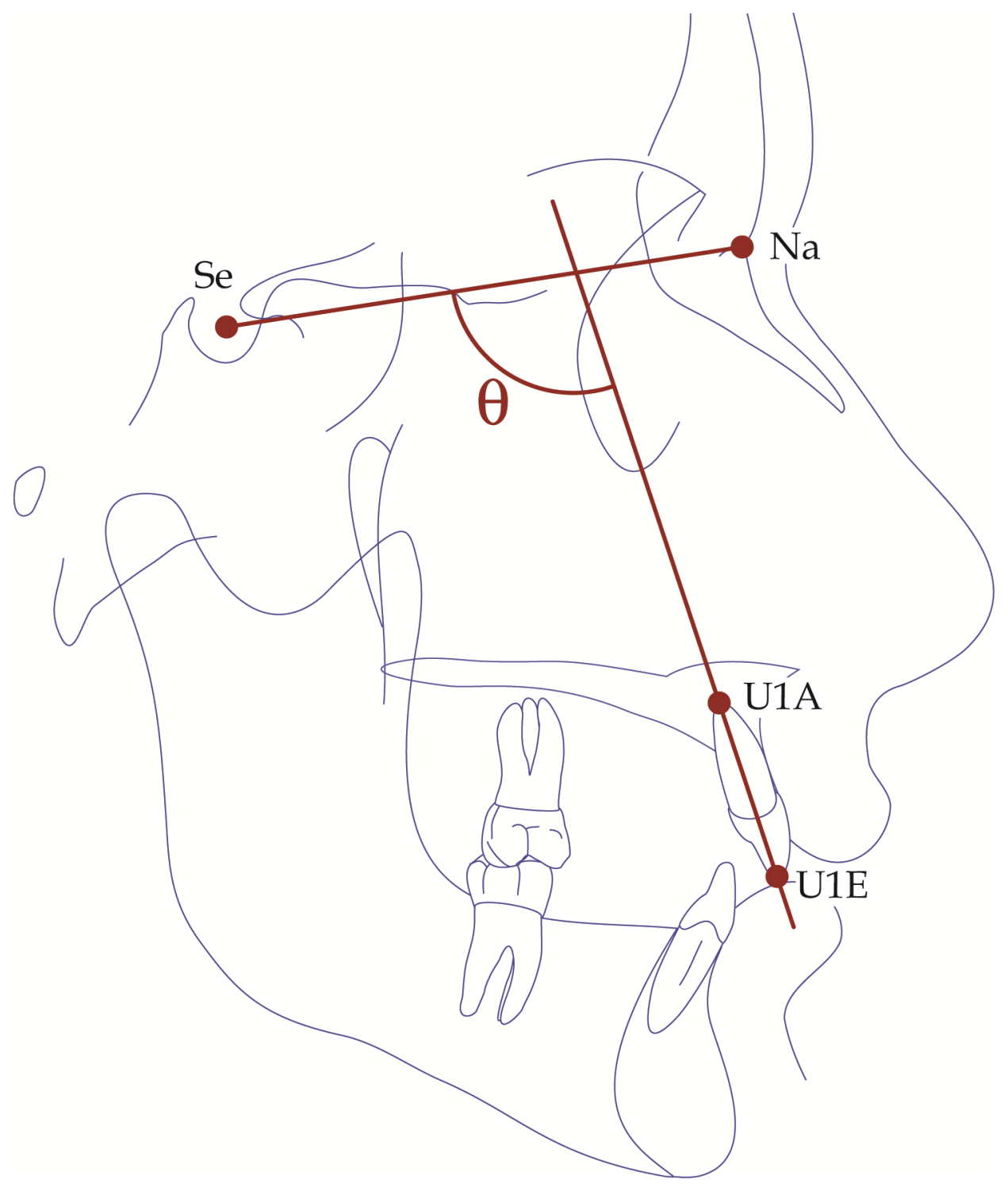

Figure D-32. Schematic tracing of a lateral cephalogram showing construction of U1-SN angle. 


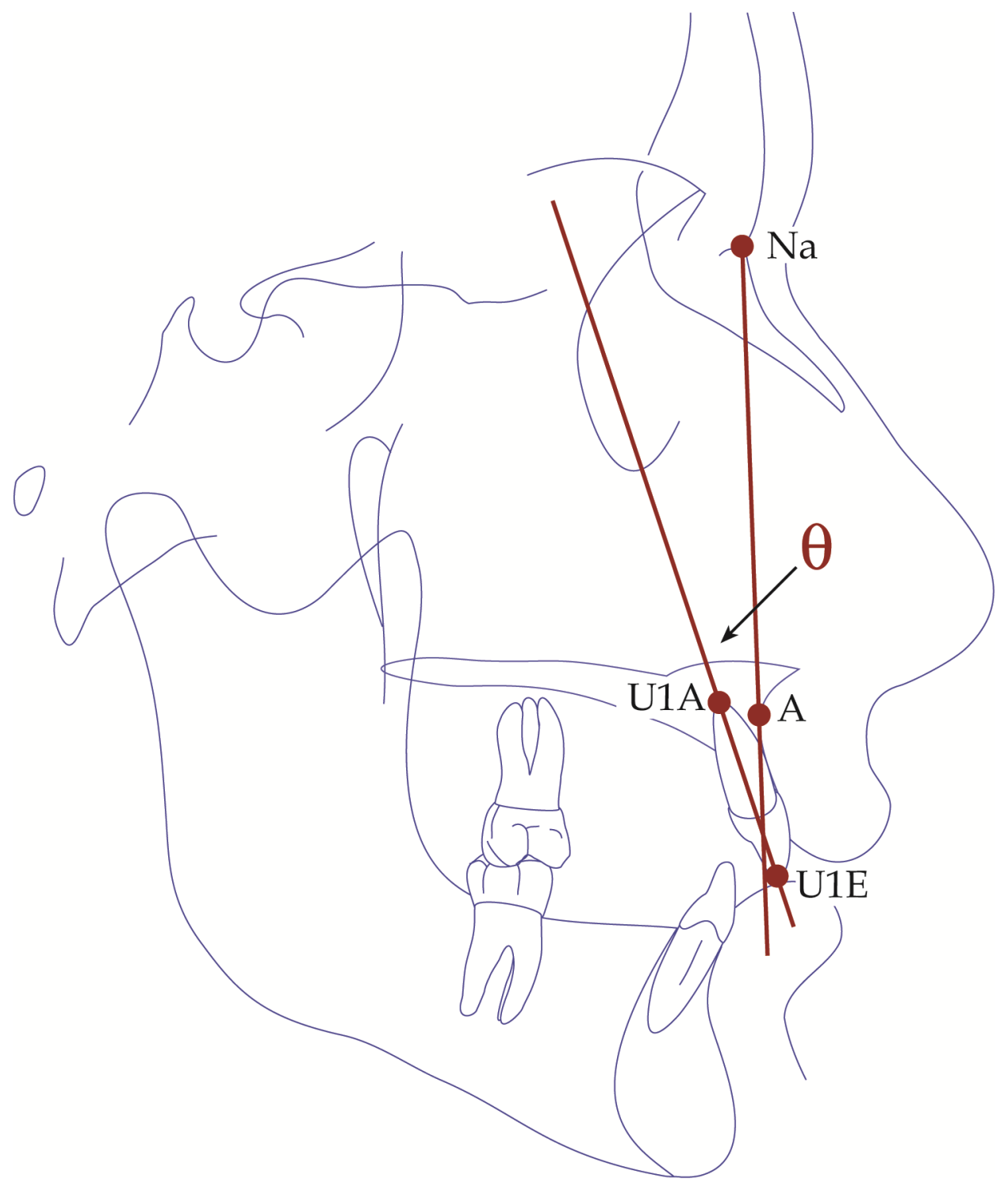

Figure D-33. Schematic tracing of a lateral cephalogram showing construction of U1-NA angle. 


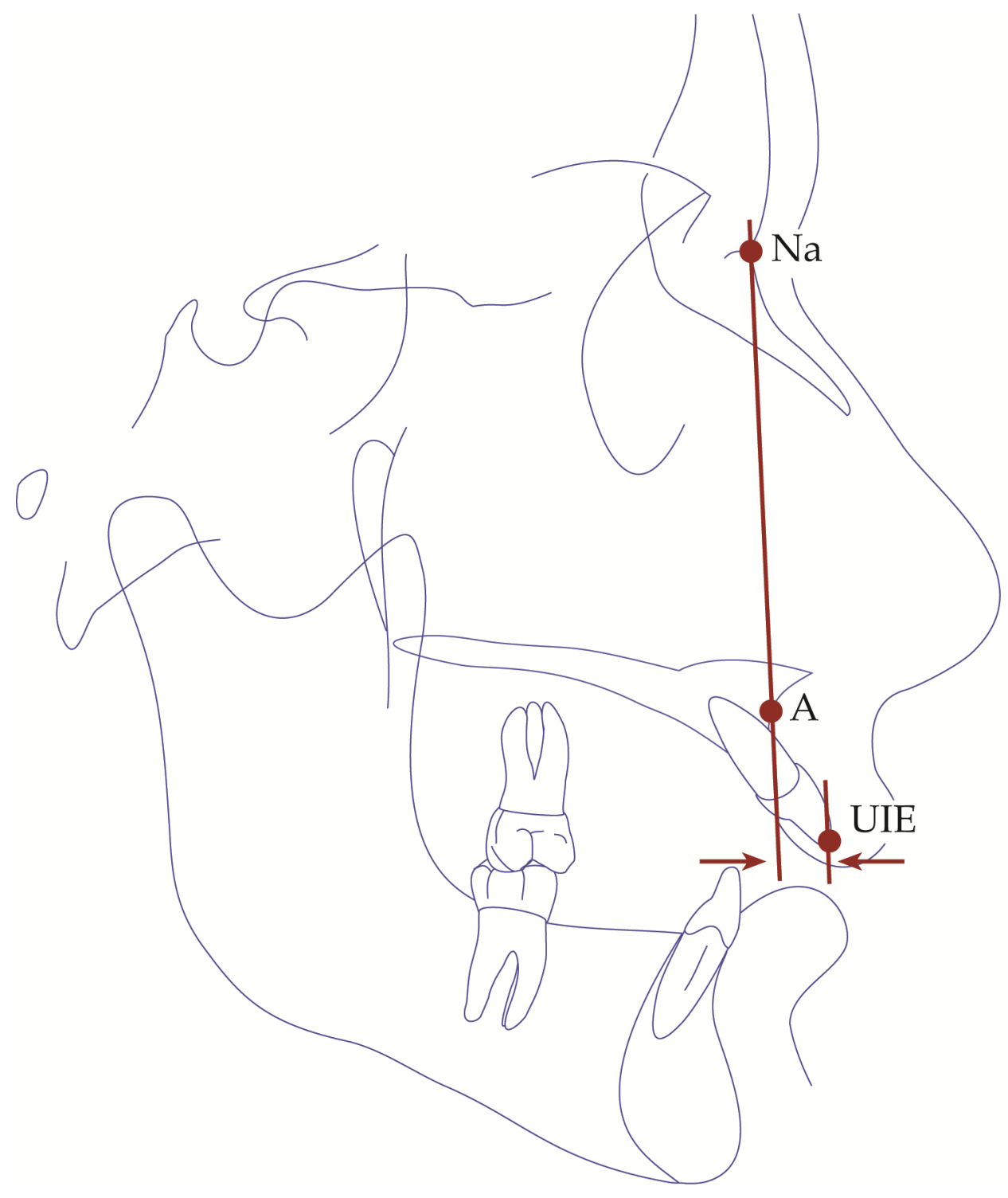

Figure D-34. Schematic tracing of a lateral cephalogram showing construction of U1-NA (mm). 


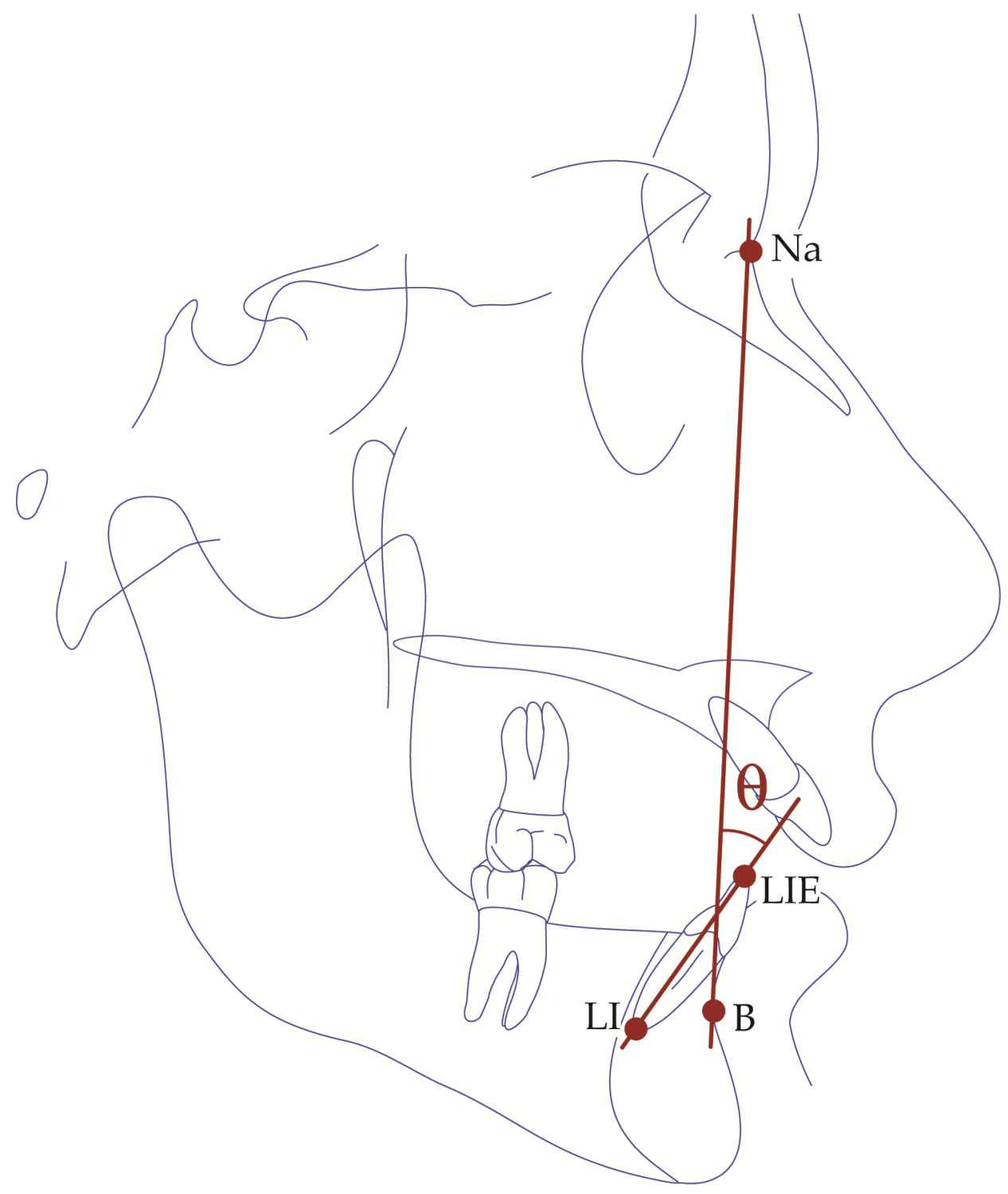

Figure D-35. Schematic tracing of a lateral cephalogram showing construction of L1-NB angle. 


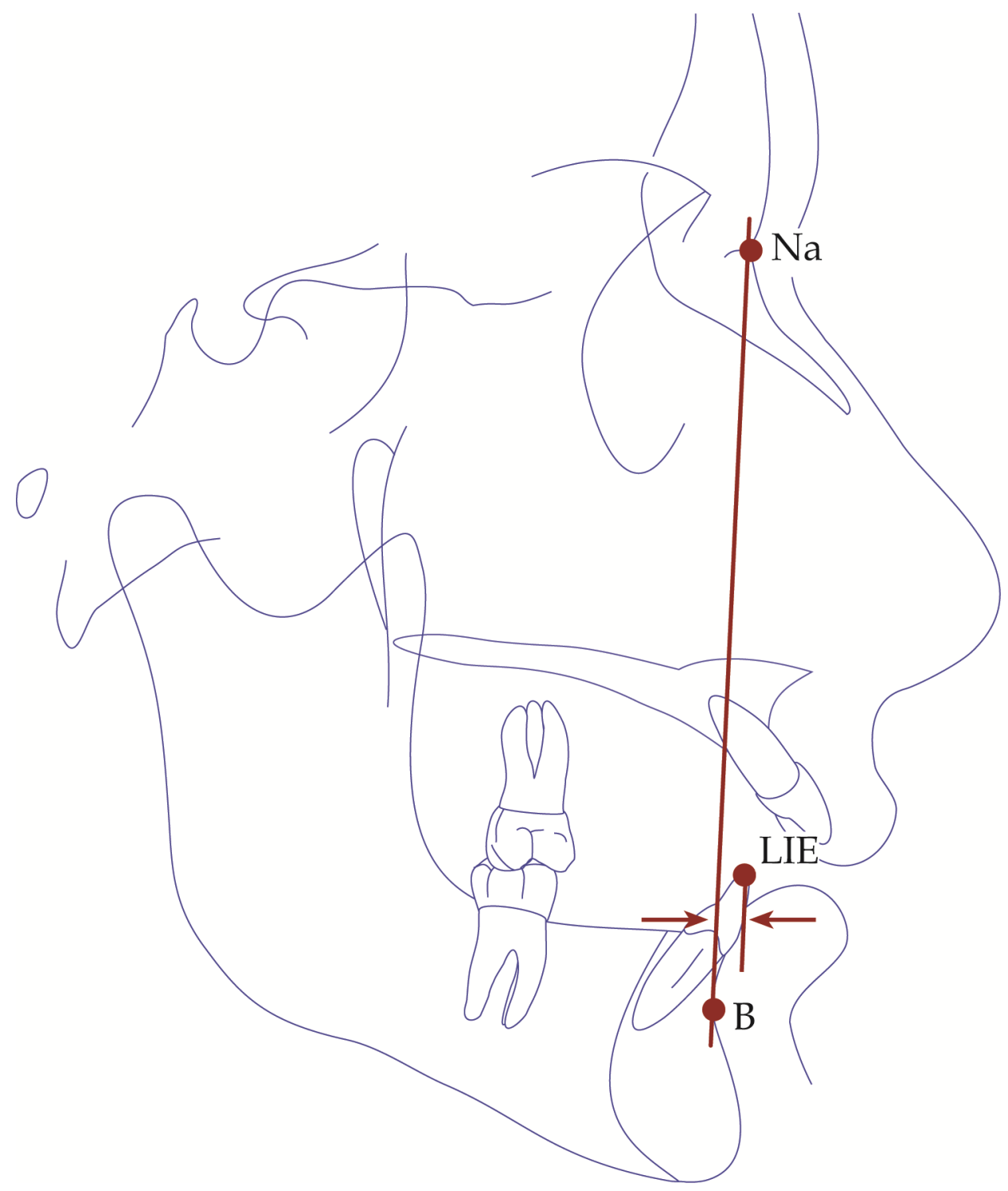

Figure D-36. Schematic tracing of a lateral cephalogram showing construction of L1-NB (mm). 


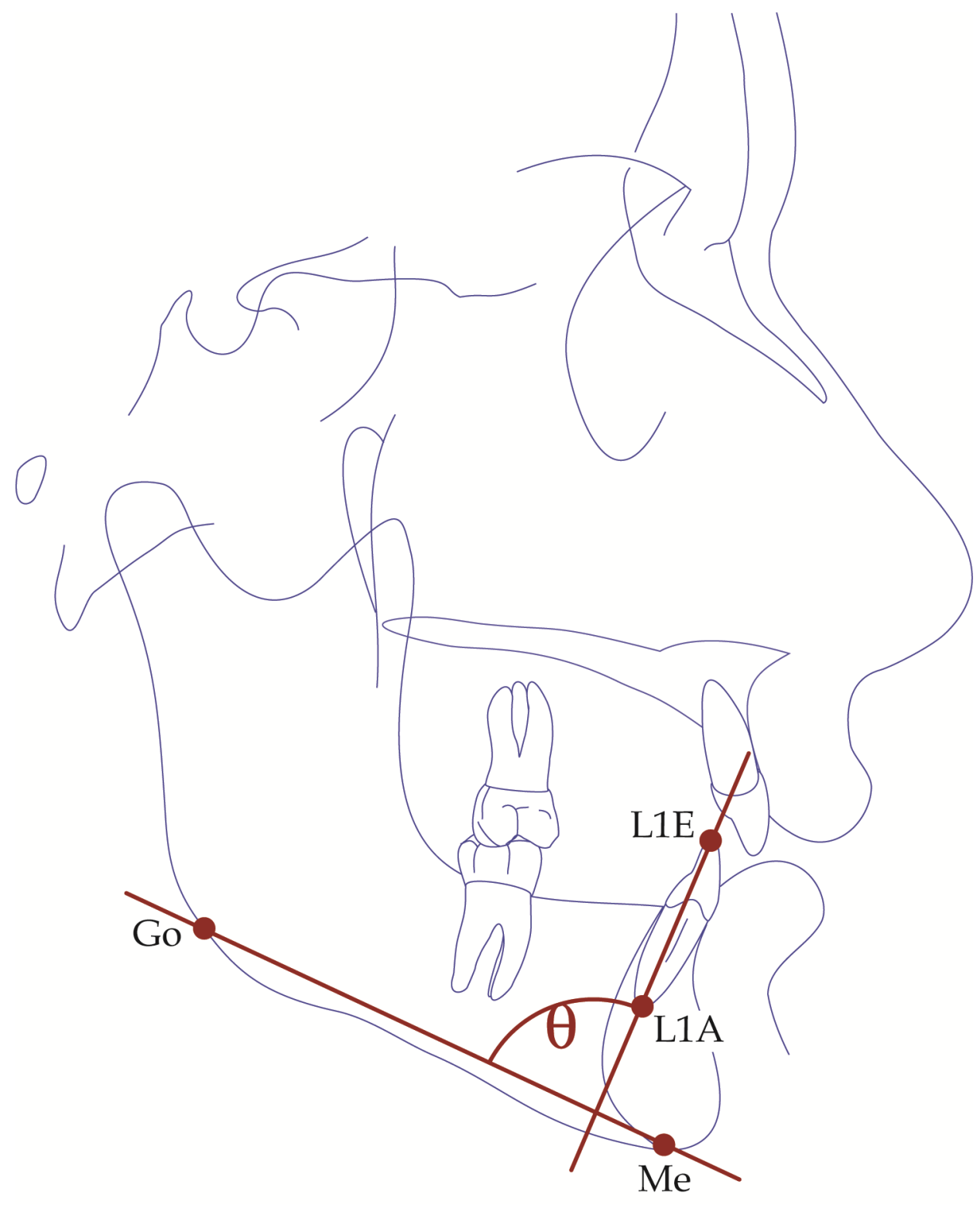

Figure D-37. Schematic tracing of a lateral cephalogram showing construction of IMPA angle. 


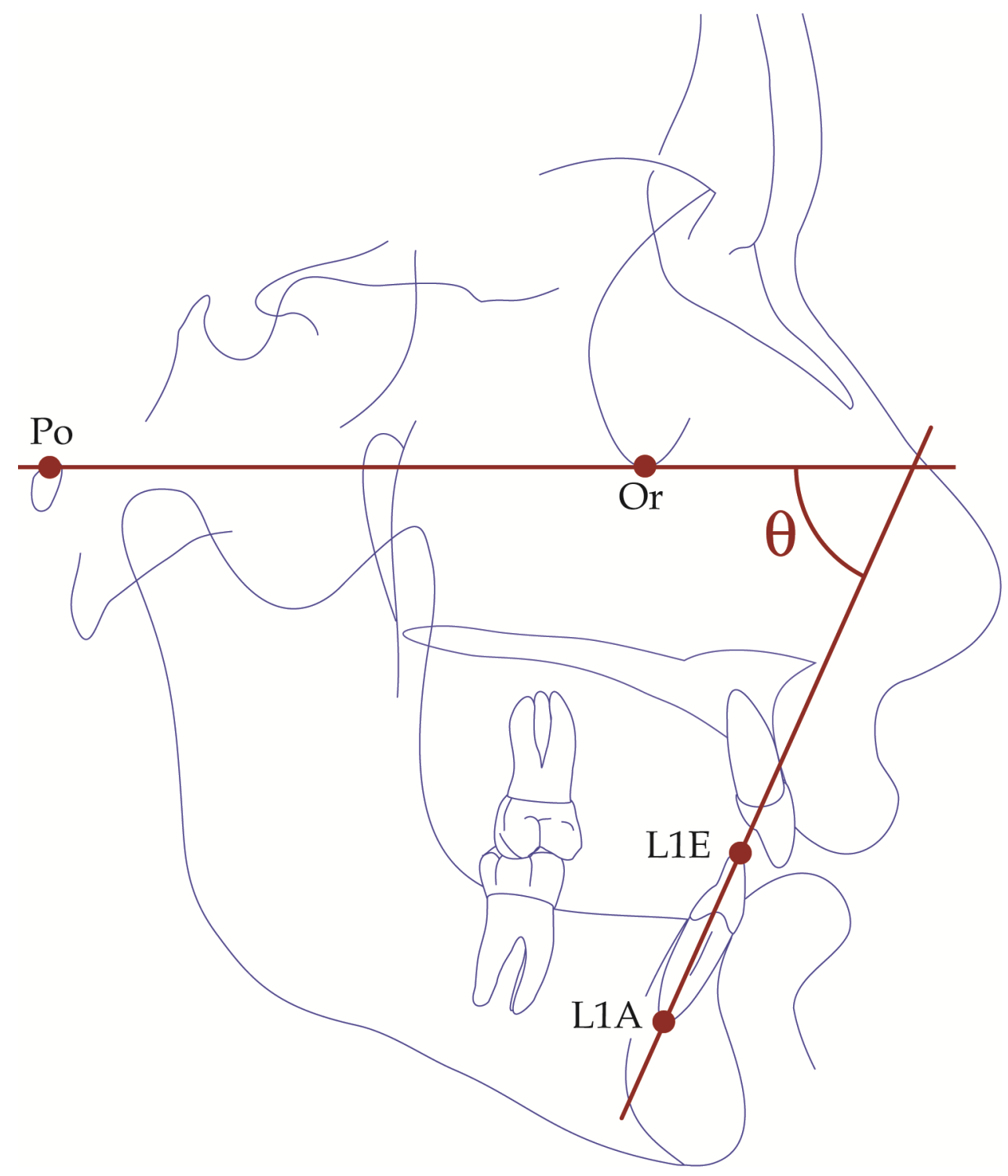

Figure D-38. Schematic tracing of a lateral cephalogram showing construction of FMIA angle. 


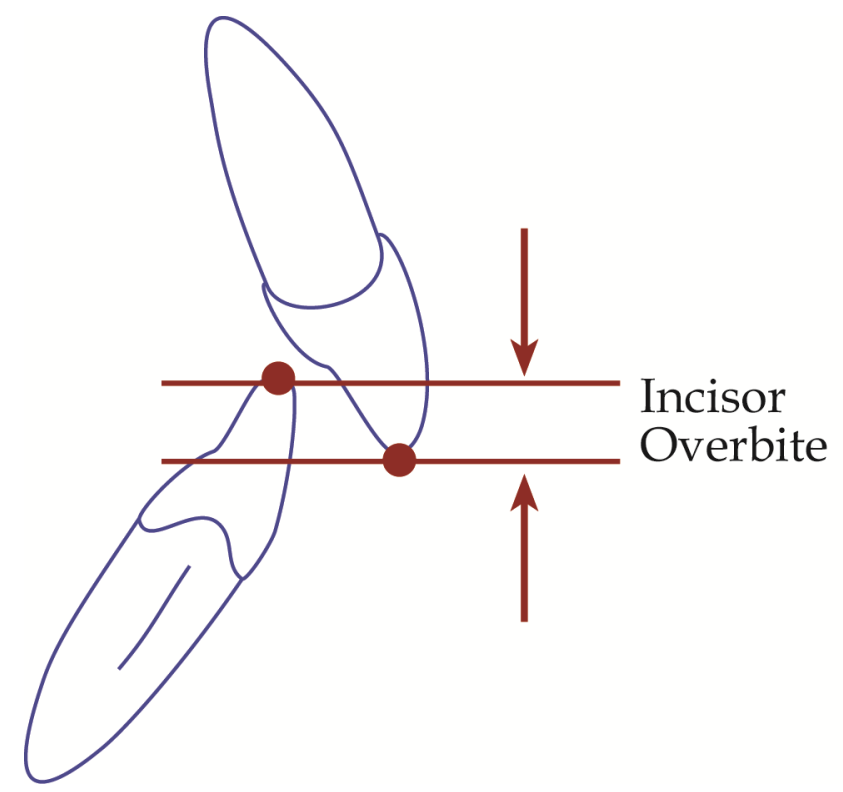

Figure D-39. Schematic tracing showing construction of Overbite (mm). 


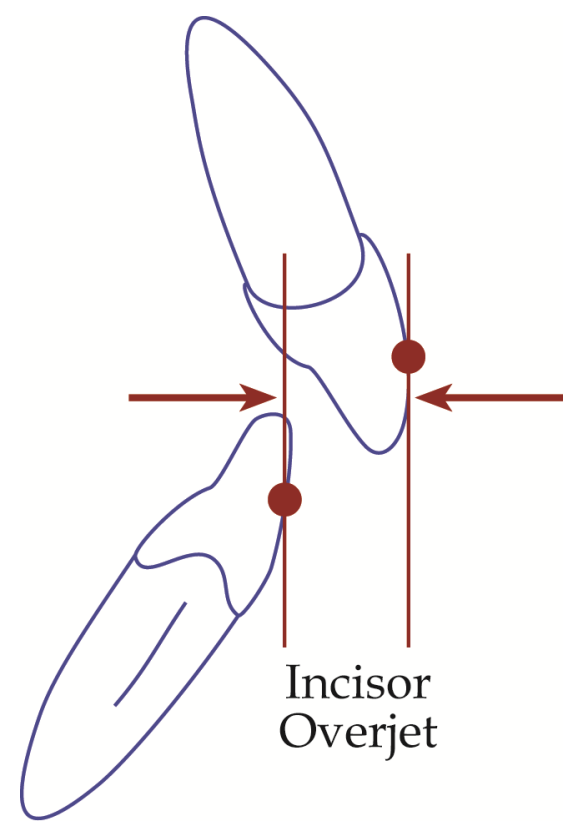

Figure D-40. Schematic tracing showing construction of Overjet (mm). 


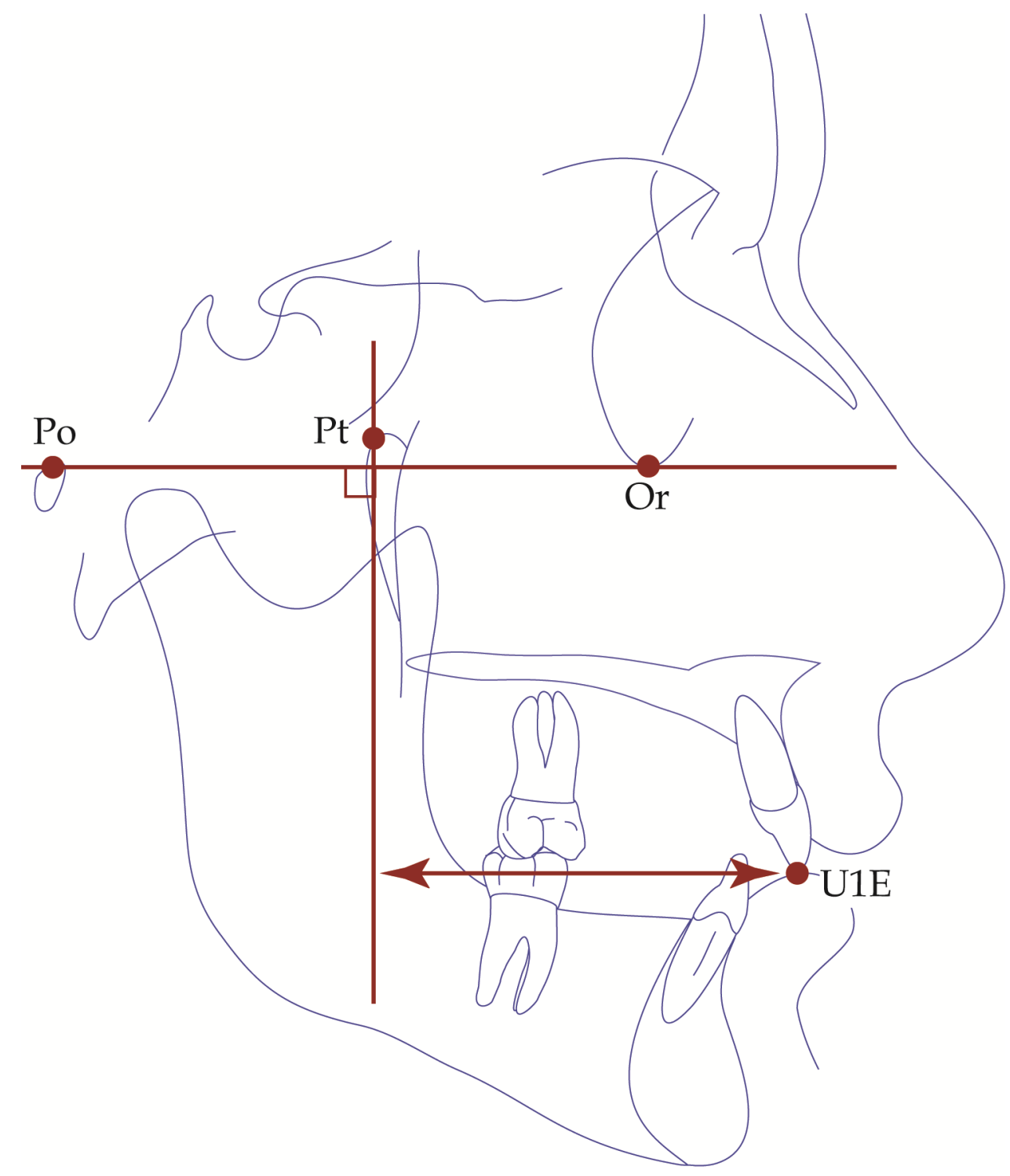

Figure D-41. Schematic tracing of a lateral cephalogram showing construction of $\mathrm{U} 1-\mathrm{PtV}$ (mm). 

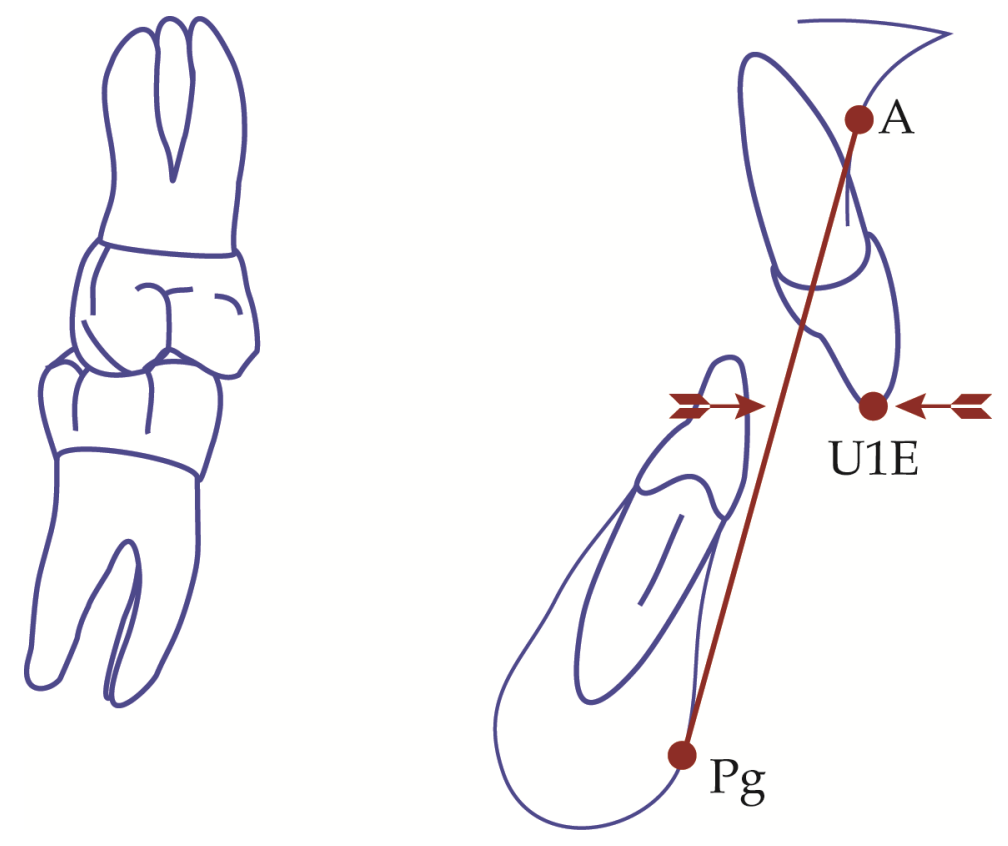

Figure D-42. Schematic tracing showing construction of U1-A-Pg (mm). 

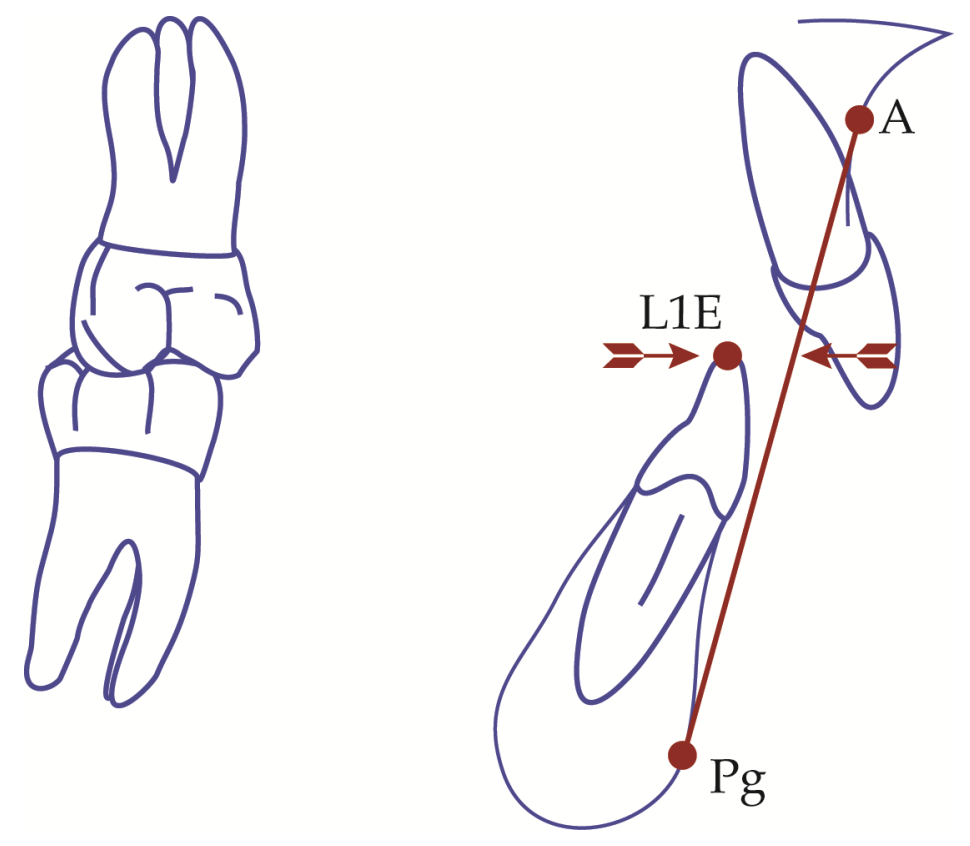

Figure D-43. Schematic tracing showing construction of L1-A-Pg (mm). 


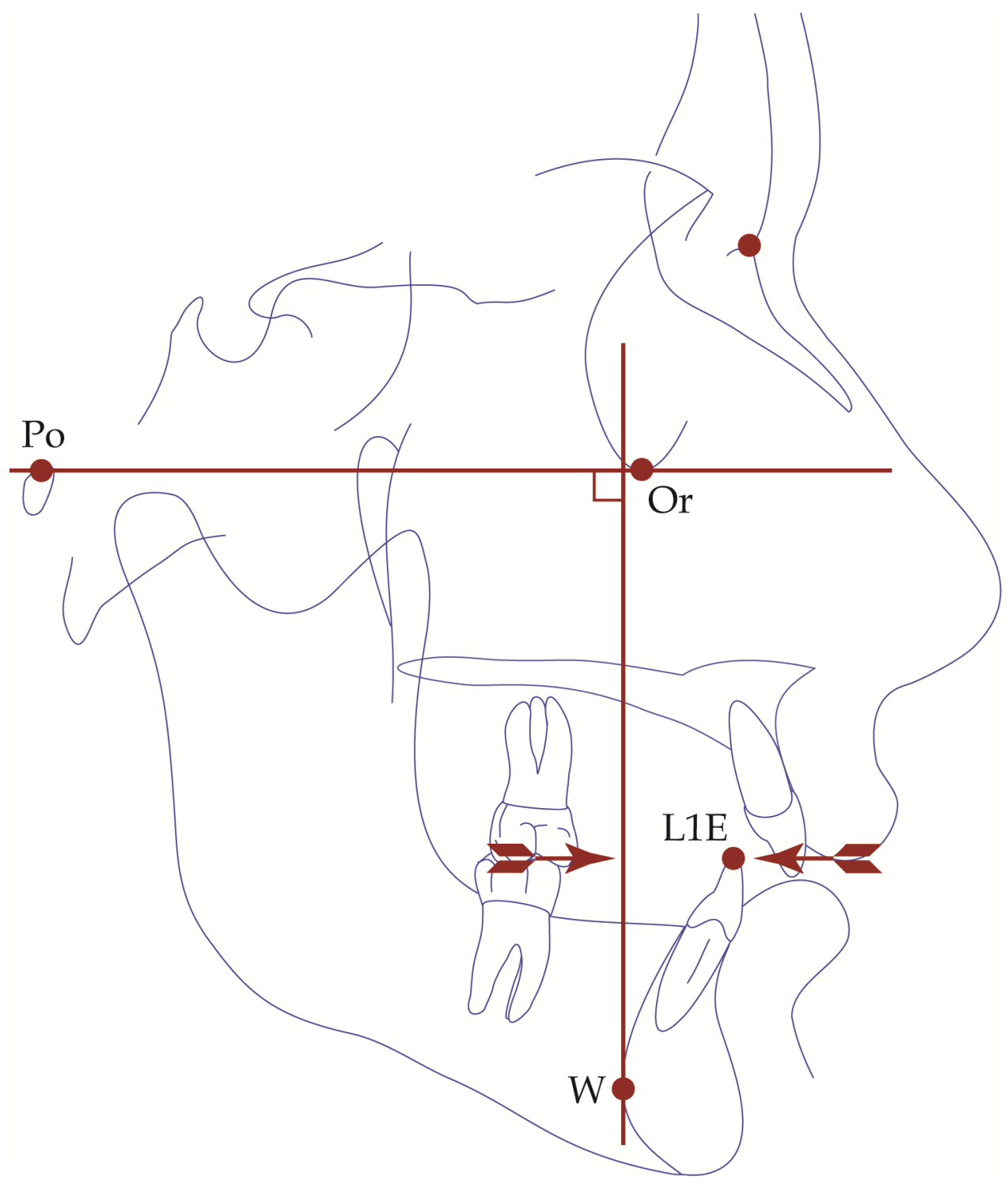

Figure D-44. Schematic tracing of a lateral cephalogram showing construction of L1-W point (mm). 


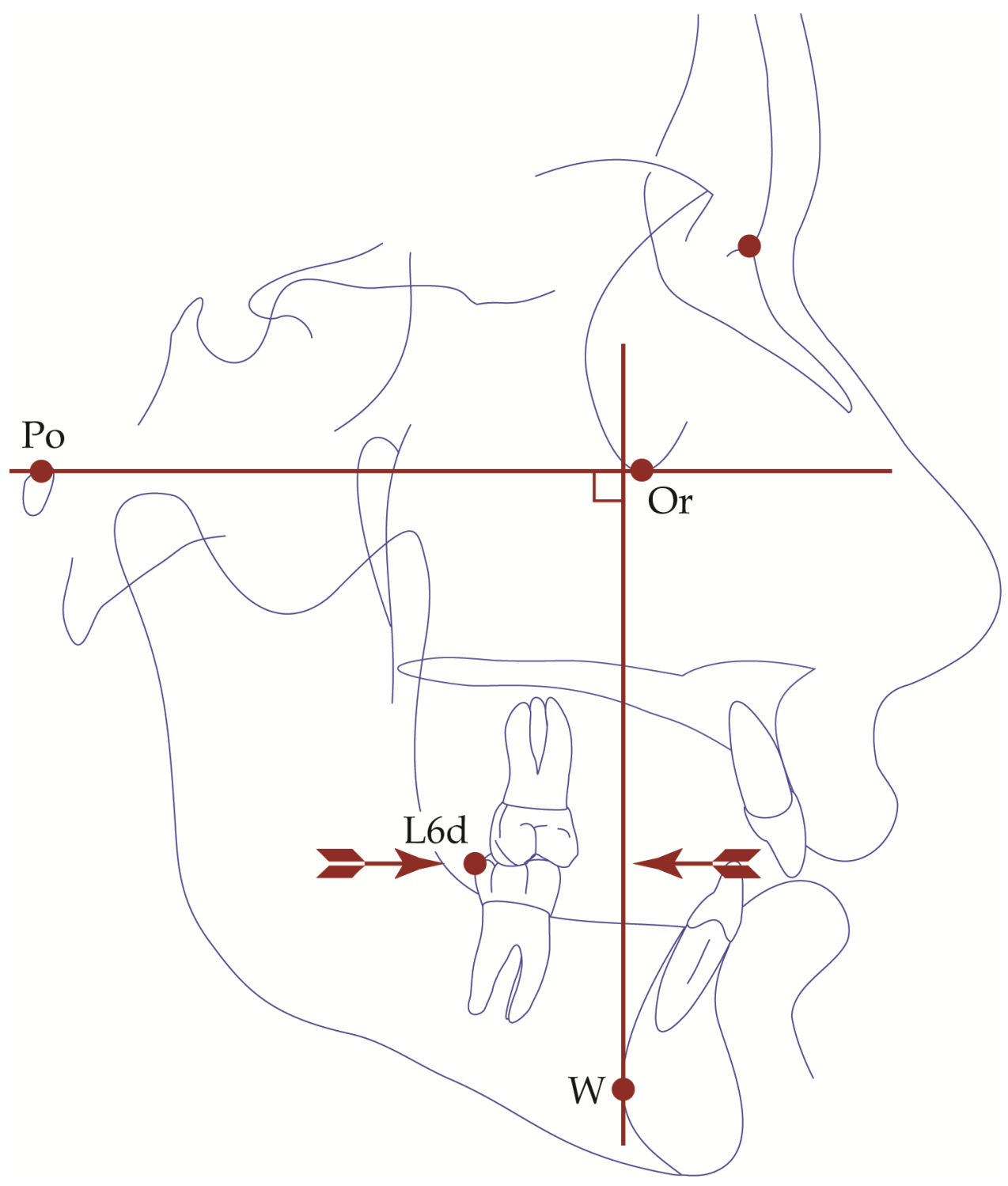

Figure D-45. Schematic tracing of a lateral cephalogram showing construction of Distal L6-W point (mm). 


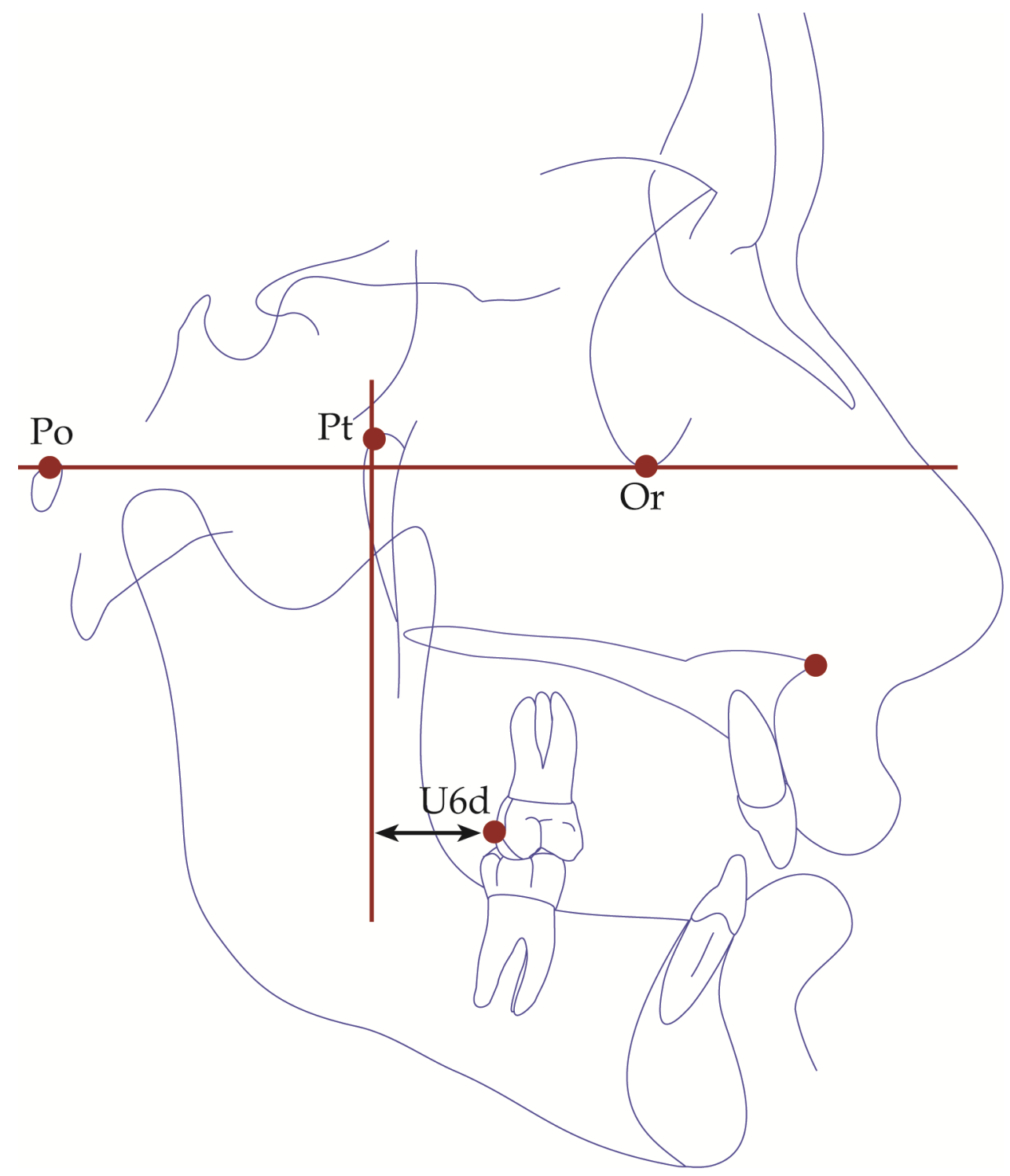

Figure D-46. Schematic tracing of a lateral cephalogram showing construction of Distal U6-PtV (mm). 


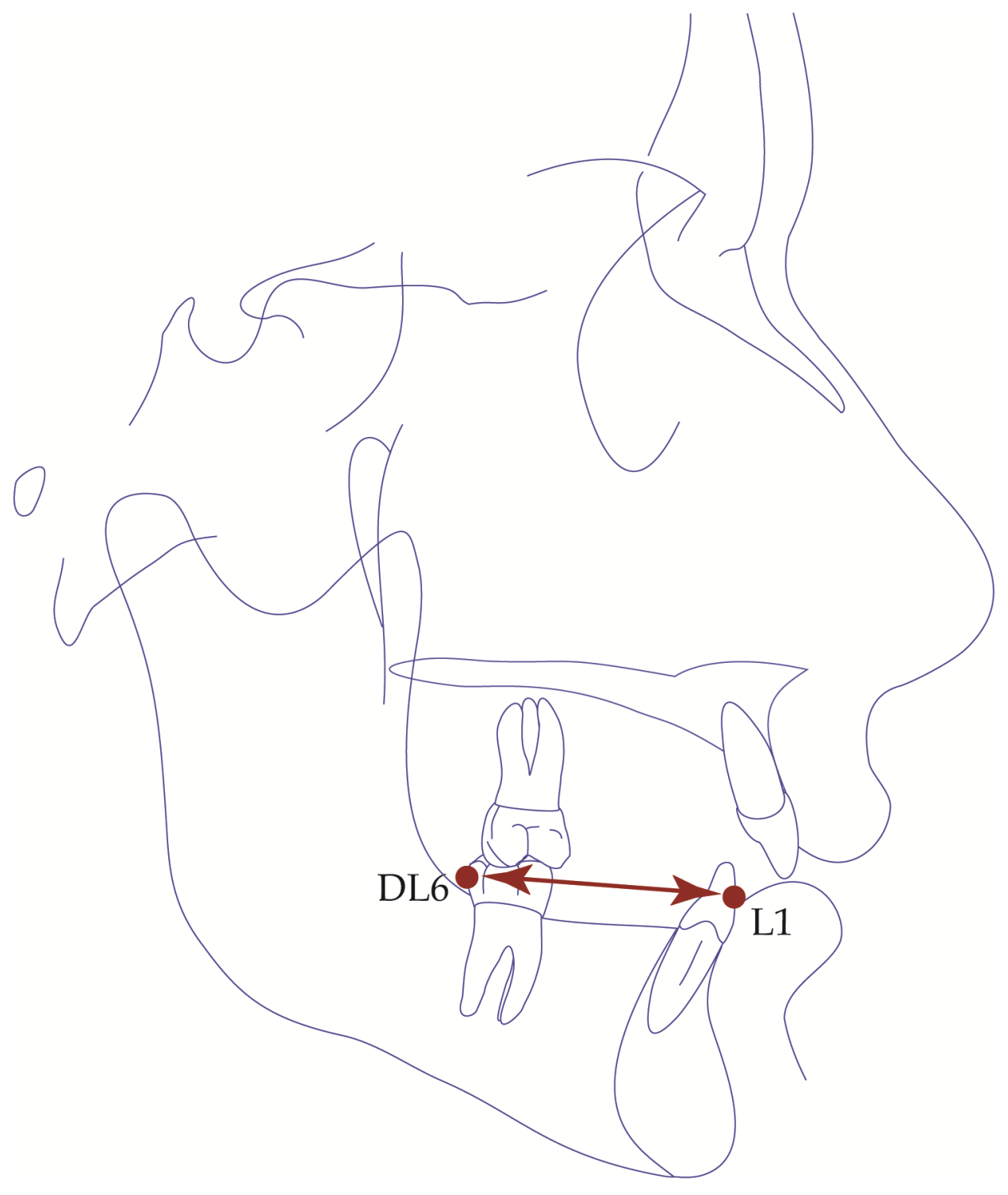

Figure D-47. Schematic tracing of a lateral cephalogram showing construction of Distal L6-L1 (mm). 


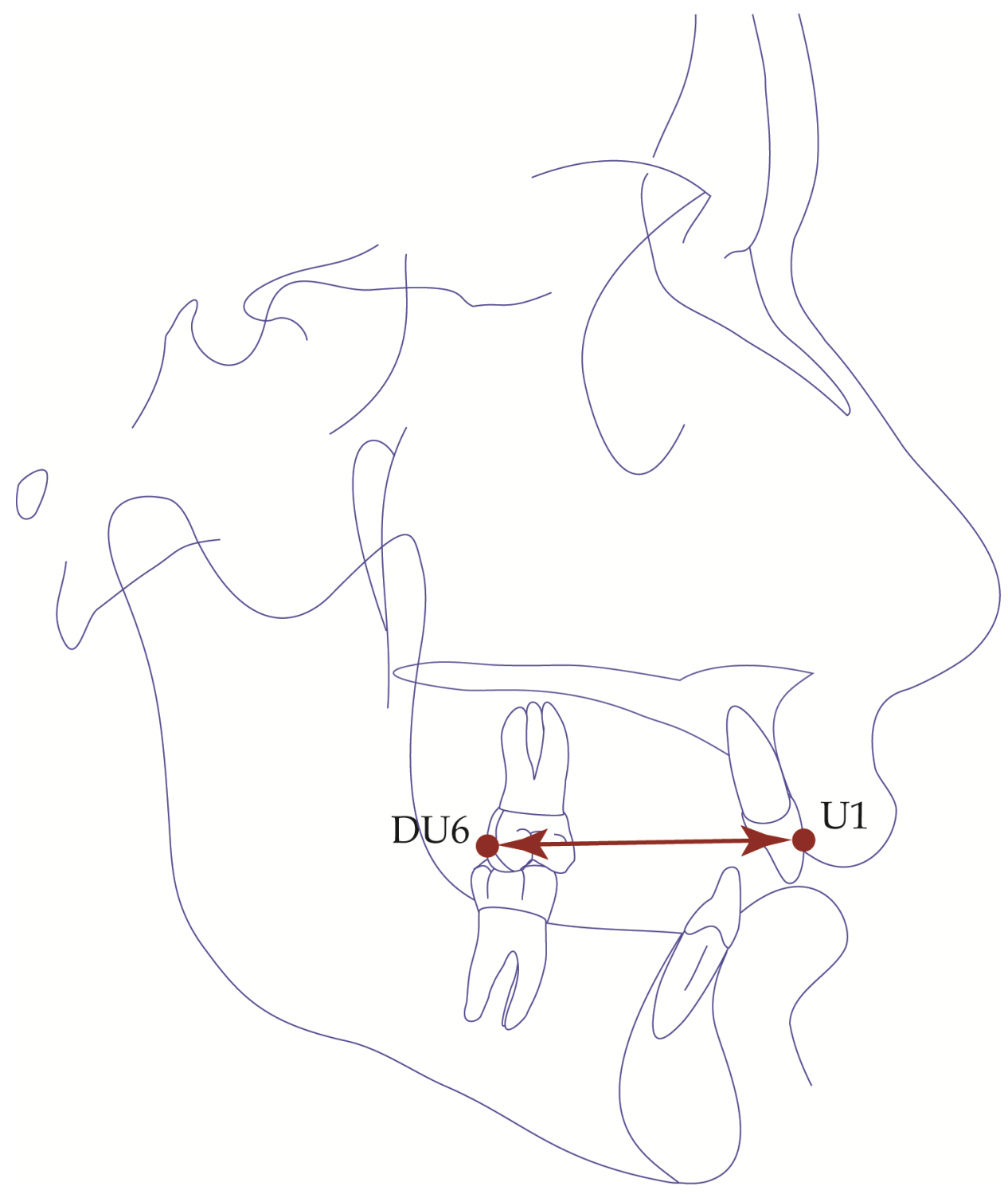

Figure D-48. Schematic tracing of a lateral cephalogram showing construction of Distal U6-U1 (mm). 


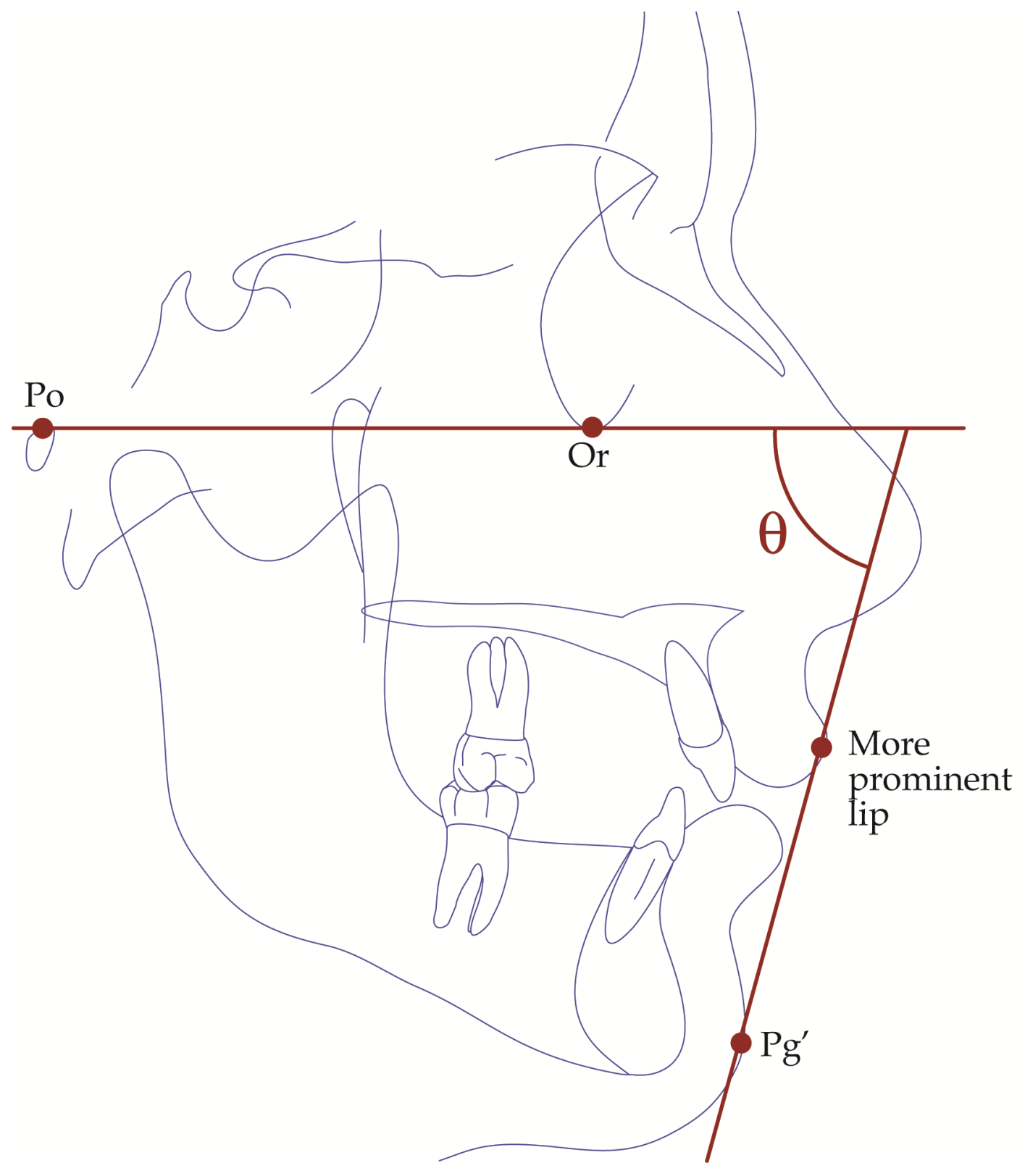

Figure D-49. Schematic tracing of a lateral cephalogram showing construction of Z Angle. 


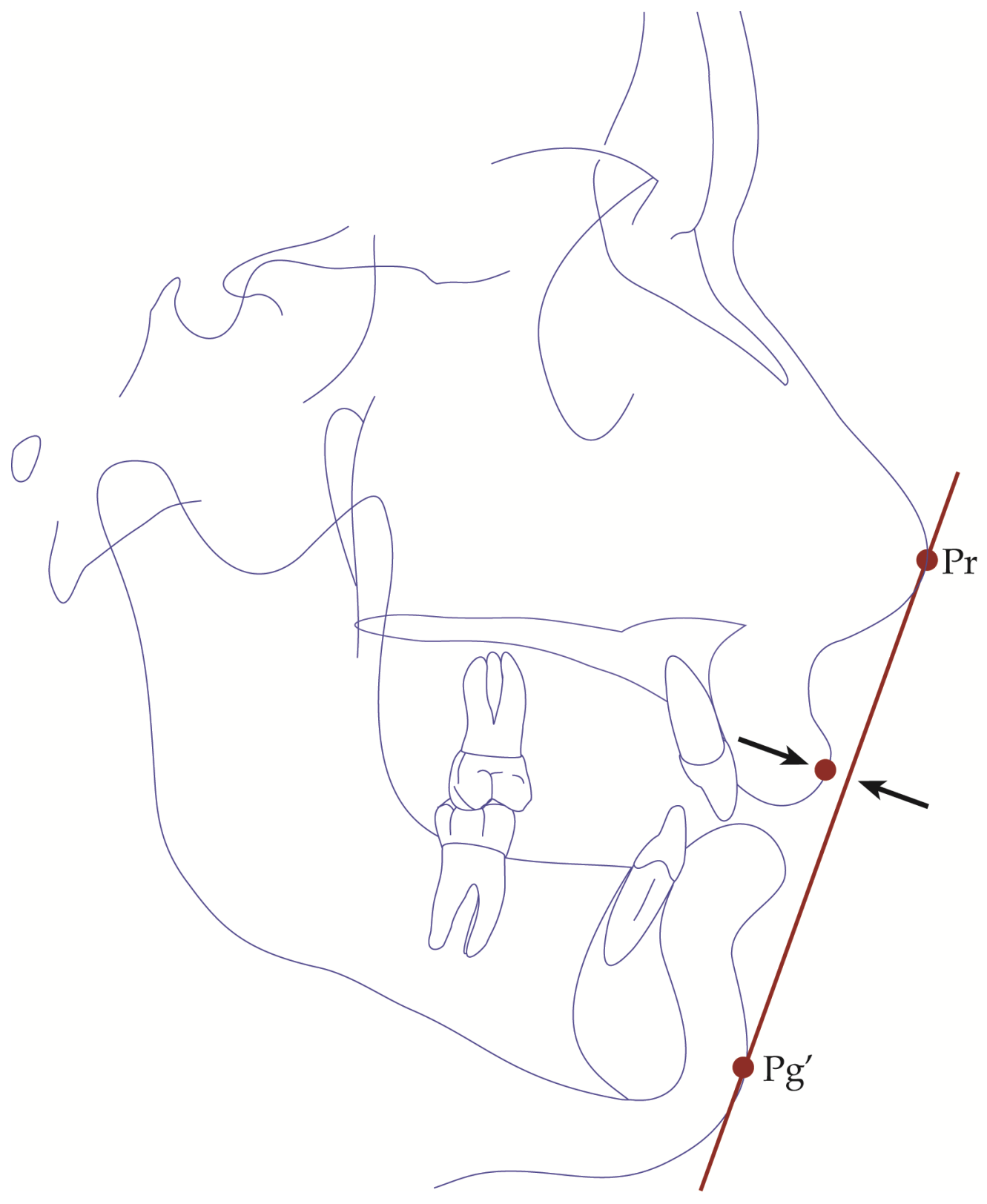

Figure D-50. Schematic tracing of a lateral cephalogram showing construction of E Plane-Ls (mm). 


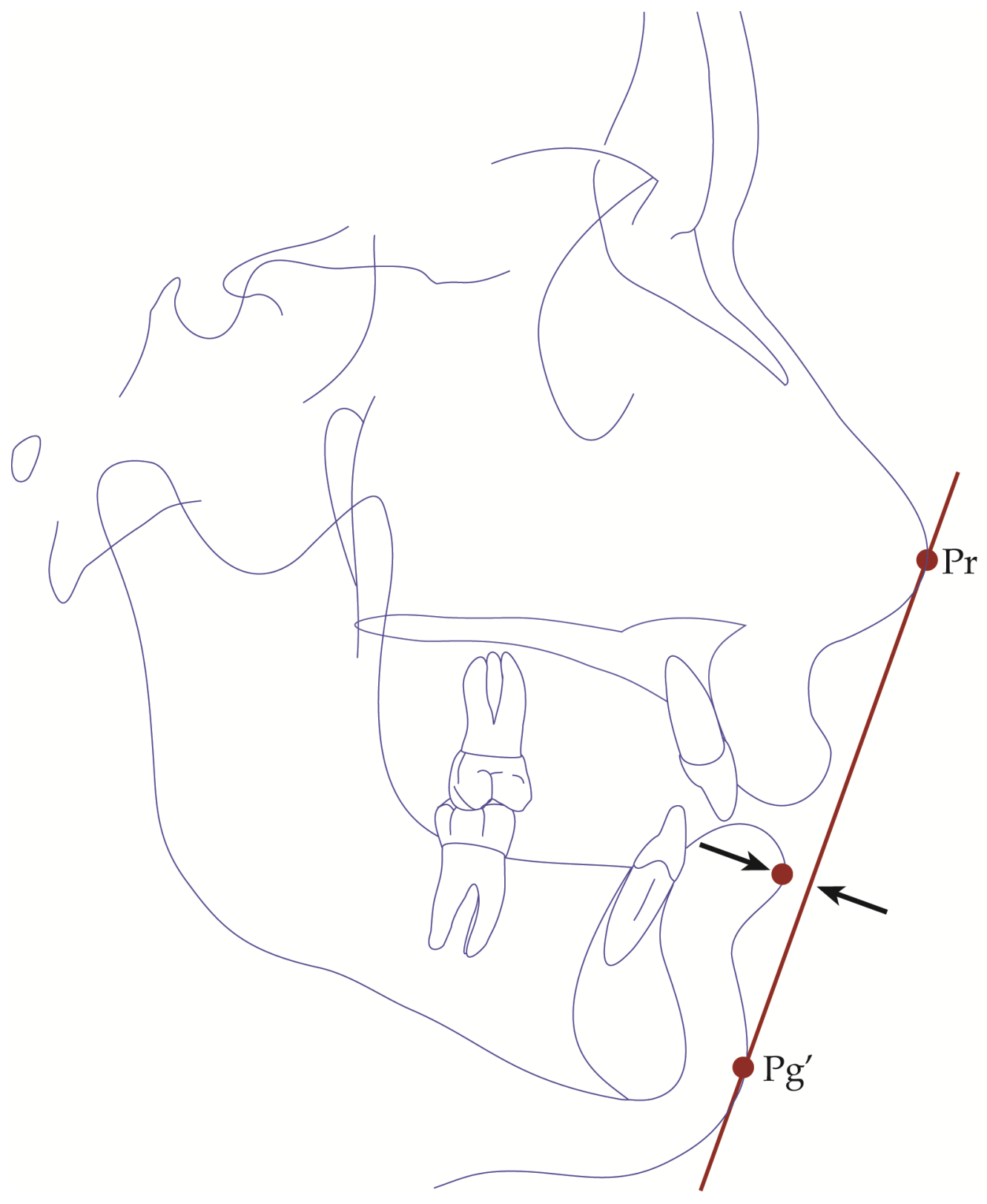

Figure D-51. Schematic tracing of a lateral cephalogram showing construction of E Plane-Li (mm). 


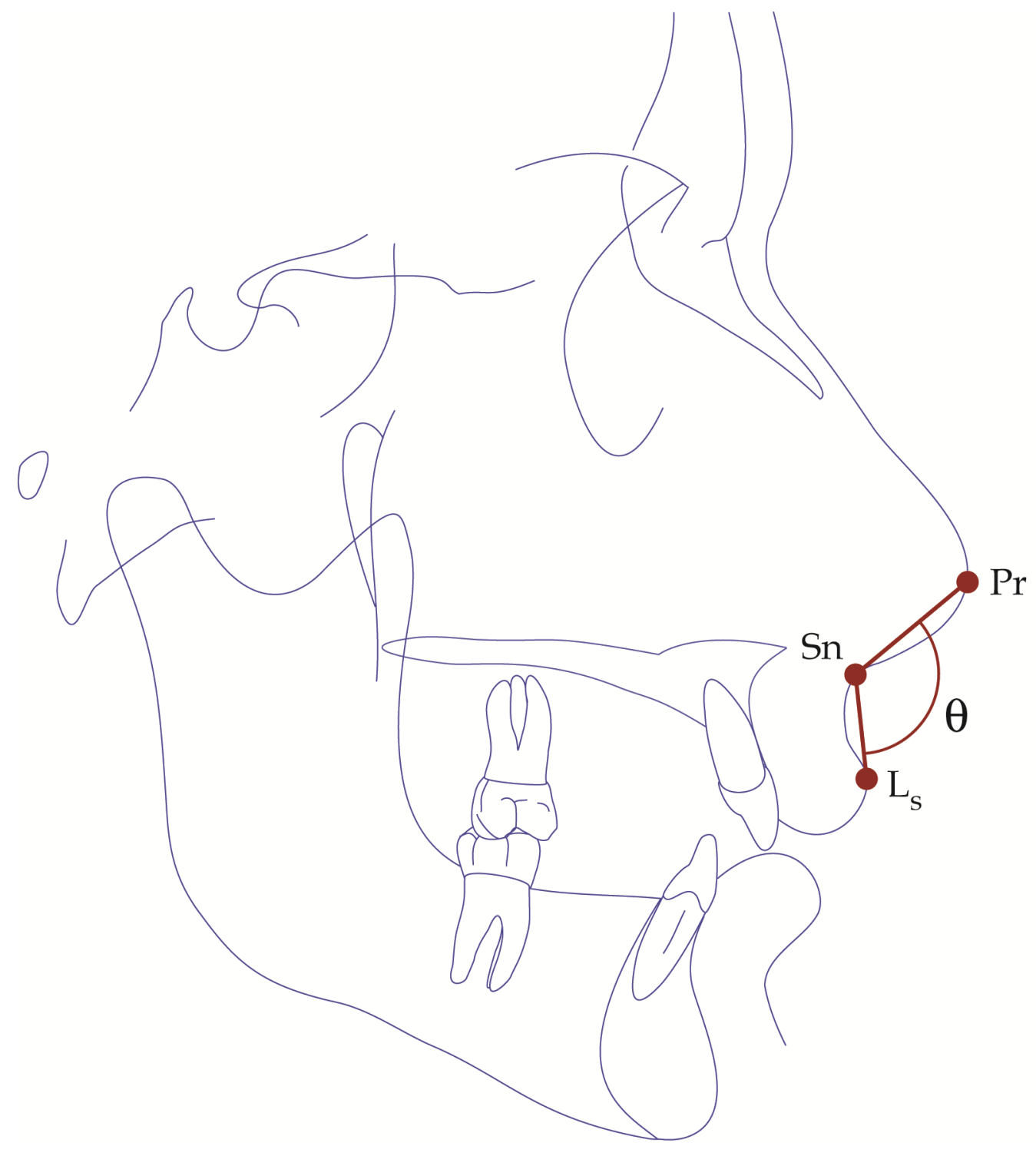

Figure D-52. Schematic tracing of a lateral cephalogram showing construction of Ls-Sn-Pr angle. 


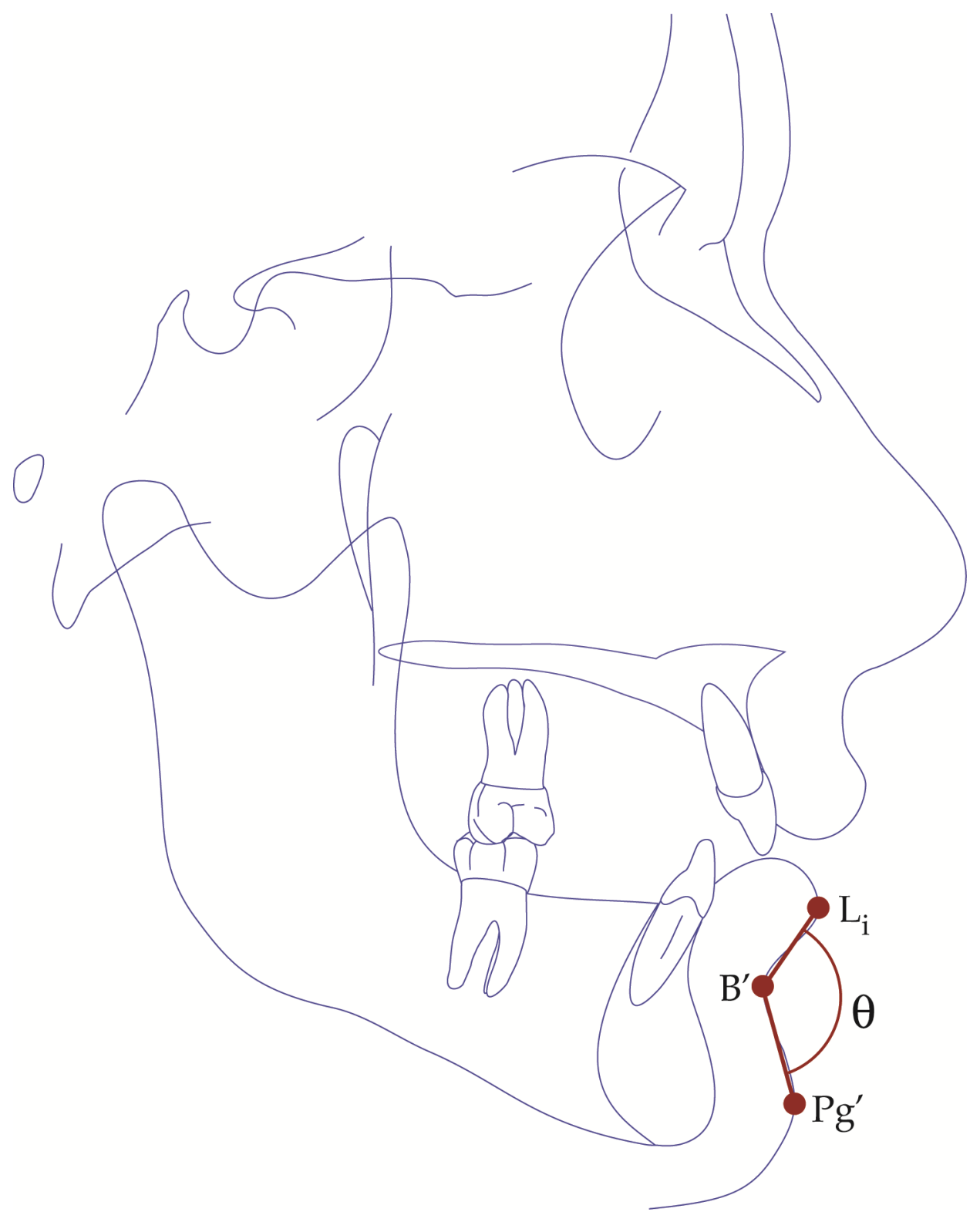

Figure D-53. Schematic tracing of a lateral cephalogram showing construction of Li-B'-Pg' angle. 


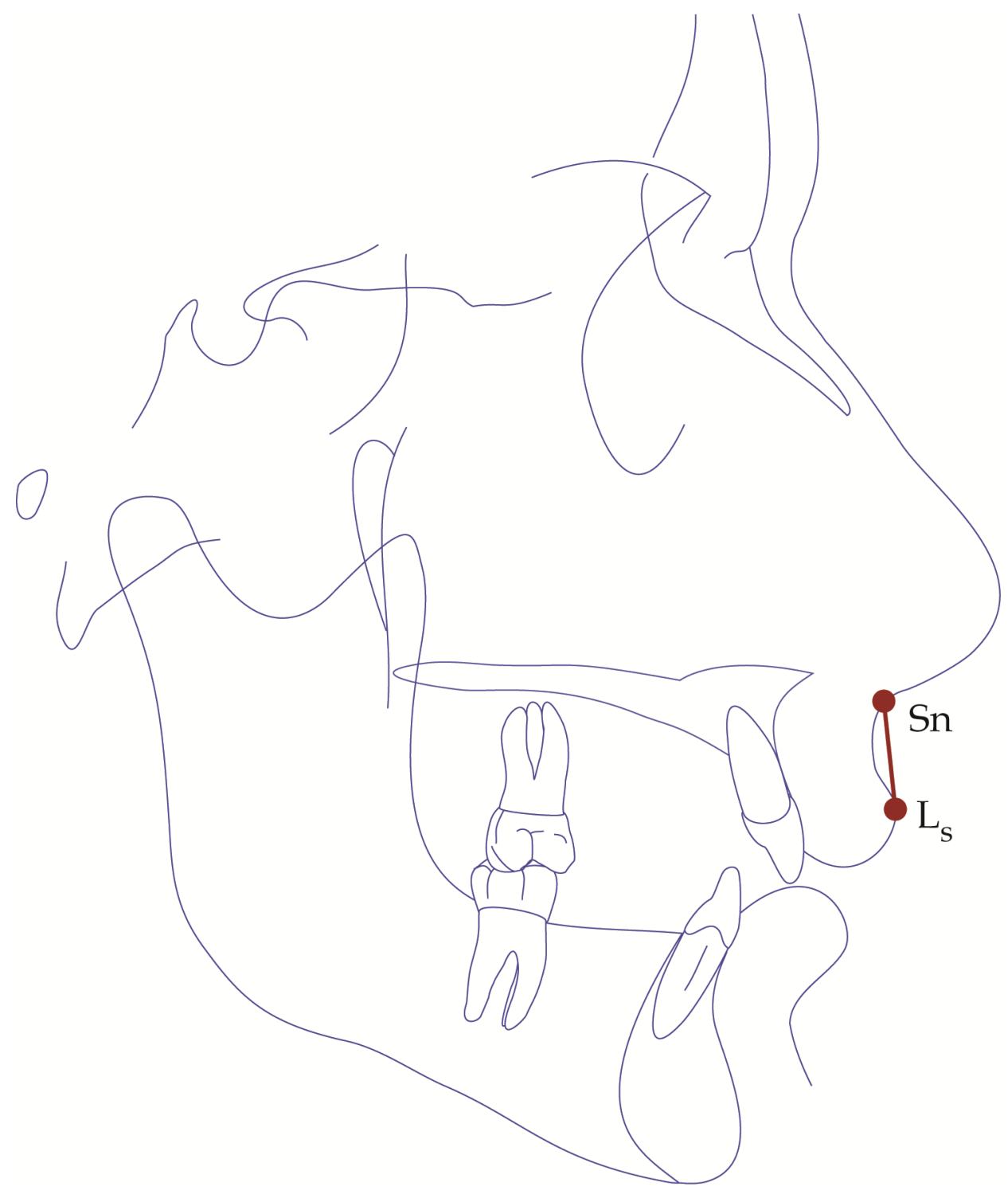

Figure D-54. Schematic tracing of a lateral cephalogram showing construction of Sn-Ls (mm). 


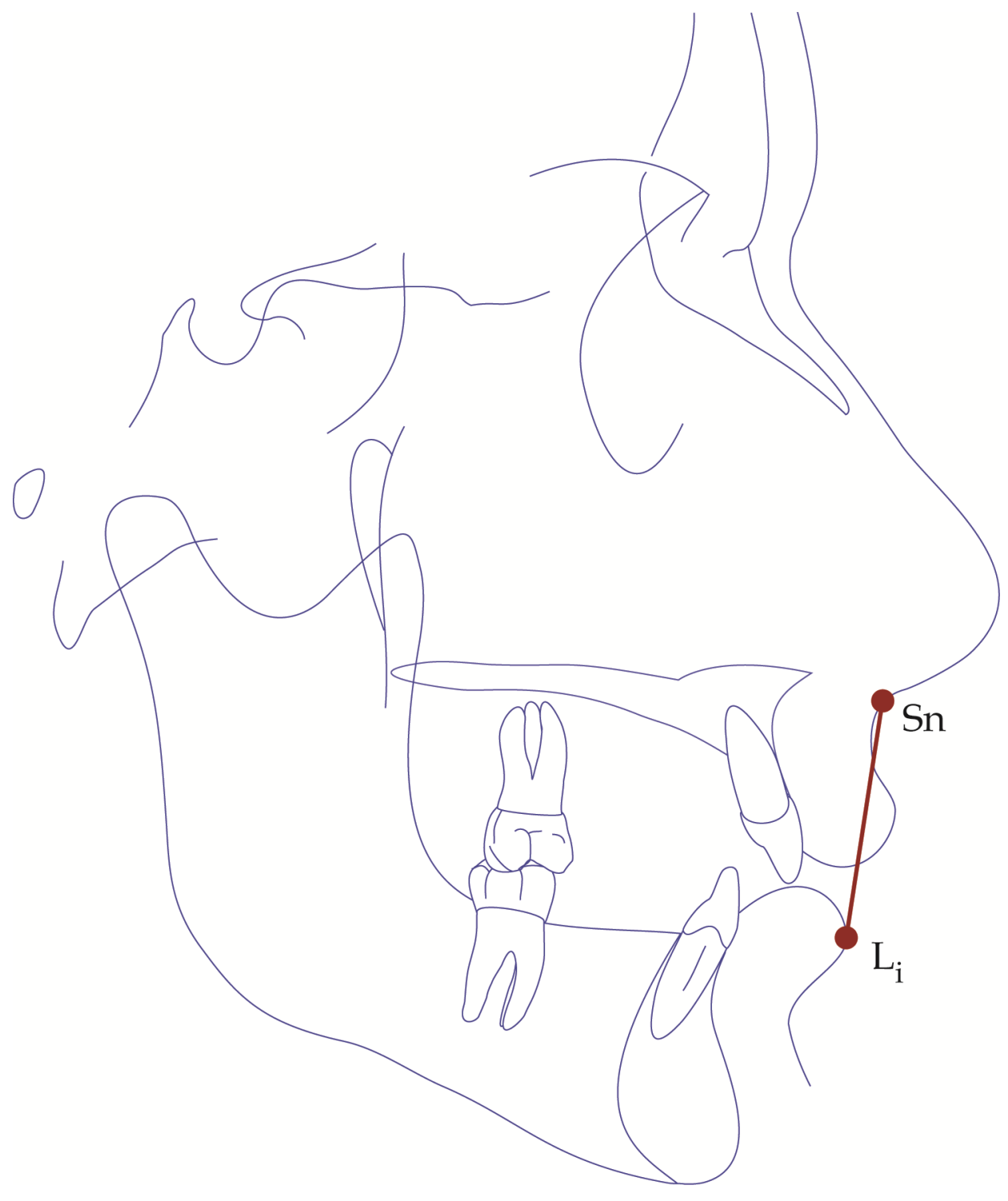

Figure D-55. Schematic tracing of a lateral cephalogram showing construction of $\mathrm{Sn}-\mathrm{Li}(\mathrm{mm})$. 


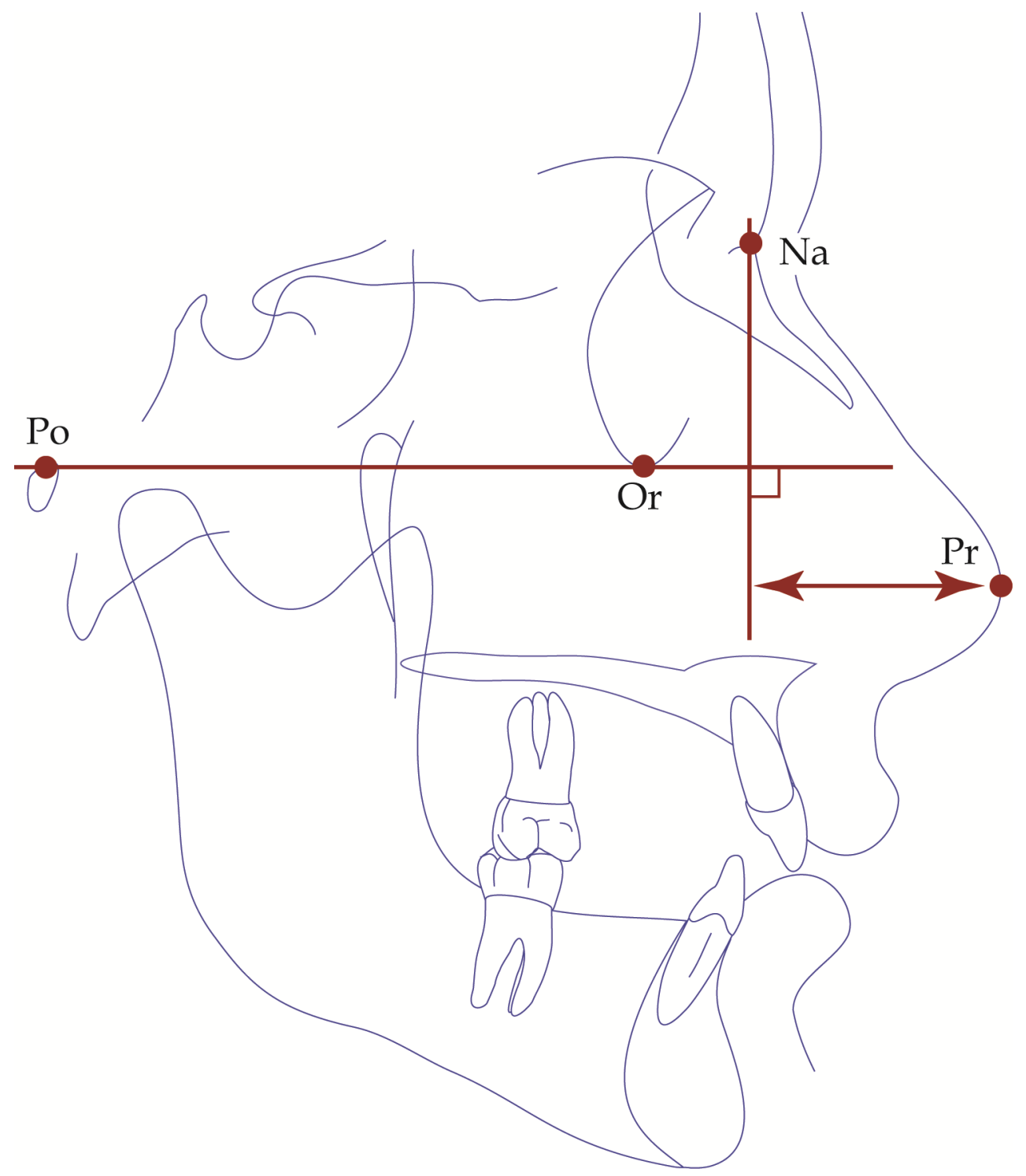

Figure D-56. Schematic tracing of a lateral cephalogram showing construction of NaPerp-Pr (mm). 


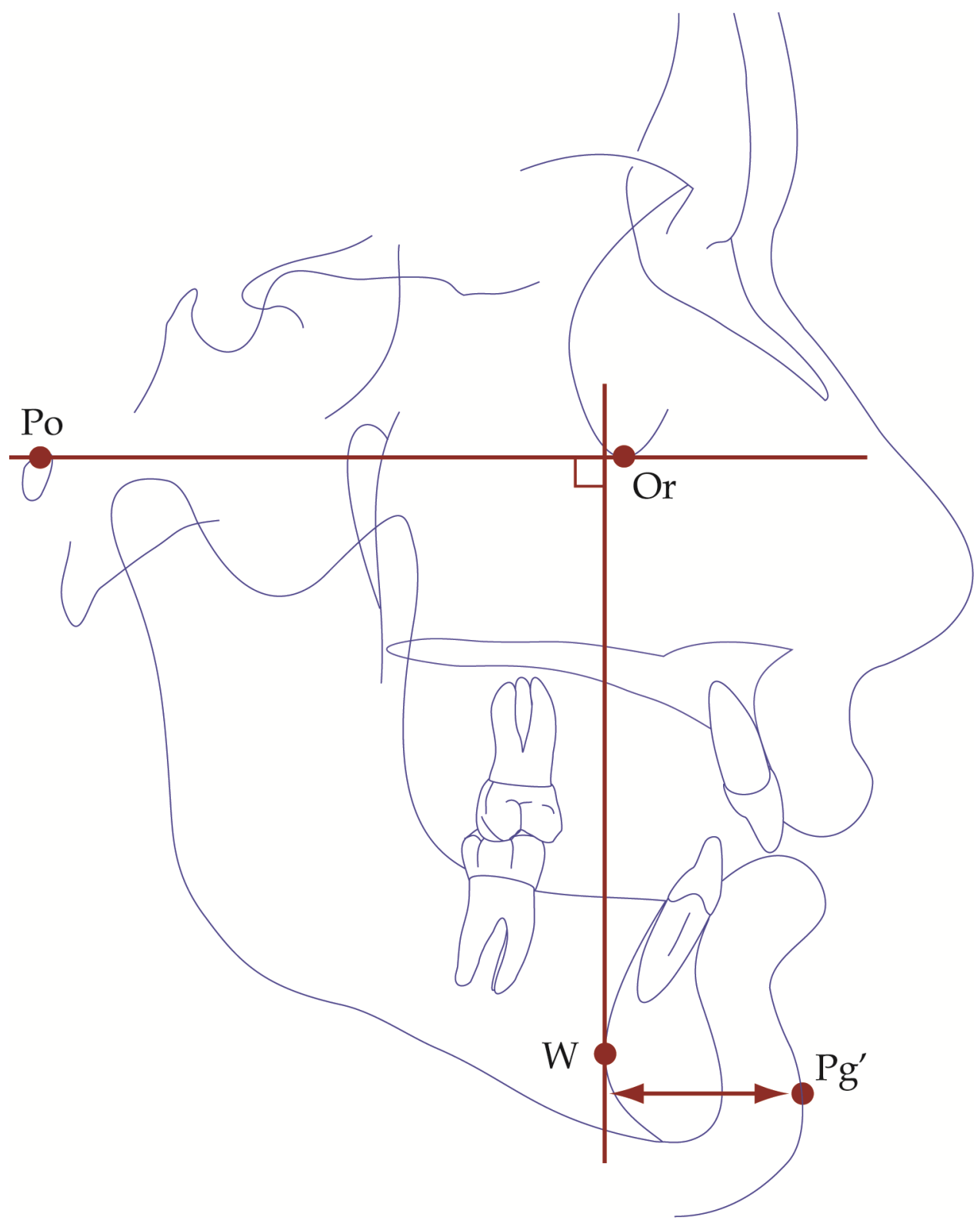

Figure D-57. Schematic tracing of a lateral cephalogram showing construction of W point-Pg' (mm). 


\section{VITA}

James Austin Rahaim was born in 1980 in Hattiesburg, MS. Austin graduated from Oak Grove High School in Hattiesburg, MS in 1998. He attended his first two years of college at the University of Southern Mississippi before transferring to Louisiana State University in 2000, where he completed his bachelor's degree in Biology in 2003. Austin was accepted to the Nova Southeastern University College of Dental Medicine in 2004, and received his Doctor of Dental Medicine degree in May 2008. In August of 2008, he became a graduate student in the Department of Orthodontics at the University of Tennessee Health Science Center and will receive his Master of Dental Science degree in May 2011. 